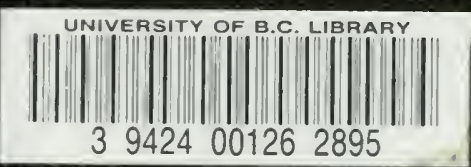

SIORAGE ITEM

F KCCESS I NG-ONE

LP1-t $20 \mathrm{E}$

U.B.C. LIBRARY 

Digitized by the Internet Archive in 2010 with funding from

University of British Columbia Library 



\section{VETERINARY COUNTER PRACTICE}


PRINTED BY

SPOTTISWOODE AND CO., NEW-STREET SQCARE LONDON 


\section{VETERINARY}

\section{COUNTER PRACTICE}

A TREATISE ON THE DISEASES OF ANIMALS AND THE MOST SUITABLE REMEDIES FOR THEM

WRITTEN EXPRESSLY FOR CHEMISTS AND DRUGGISTS

BY QUALIFIED AND EXPERIENCED .MEMBERS OF

THE ROYAL COLLEGE OF VETERINARY SURGEONS

THIRD EDITION

Dublisbec at the Offices of

'THE CHEMISTAND DRUGGIST'

42 CANNON STREET, LONDON

AND AT MELBOURNE AND SYDNEY, AUSTRALASIA 



\section{PREFACE}

THis book has been written because it is considered that Chemists and Druggists do not obtain that share of the reterinary practice of the country to which their knowledge of drugs and their action might fairly entitle them. While they could not, and need not, desire to compete with the qualified Veterinary Surgeon, they might, to a much greater extent than they do, supply the passing requirements of the farmer, the stockholder, and the pet owner. These are at present dependent to a considerable extent on a class of men whose practice, successful though it often is, is always empirical, and not seldom dangerous. The suggestions comprised in the following pages will not serve as a substitute for the clinical experience absolutely necessary to the equipment of a competent veterinary practitioner, but in hundreds of cases they will enable the chemist to understand cases detailed to him at his counter, and to supply the most suitable remedy. The work has been written throughout by examined and experienced veterinary surgeons. A few of the chapters have already appeared in 'The Chemist and Druggist,' and these have all been carefully revised. The greater part of the book, however, is entirely new.

April ISgI.

For this third edition the work has been thoroughly revised by the member of the Royal College of Veterinary Surgeons who originally prepared it, and many illustrations and much new matter have been introduced.

June 1900. 



\section{CONTENTS}

PAGE

MEDICAL AND SURGICAL TREATMENT OF DOMESTIC ANIMALS - I

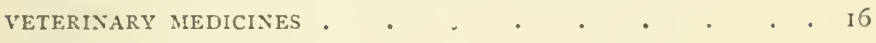

DISEASES OF THE HORSE . . . . . . . . . 59

LAMENESS IN HORSES • • • • • • • . . II9

DENTITION OF THE HORSE . . . . . . . . I47

Diseases OF CATtLE . . . . . . . . . . I53

DISEASES OF SHEEP $\quad$. . . . . . . . . . . I99

DISEASES OF PIGS . . . . . . . . . . . . 216

DISEASES OF DOGS . . . . . . . . . . . . . 226

TREATMEXT OF EYE DISEASES . . . . . . . . 259

WOUNDS, SORES, ETC., IN ANIMALS . . . . . . 267

DISEASES OF POULTRY . . . . . . . . . . . . 286

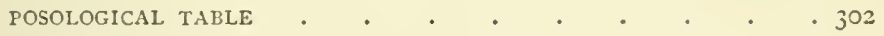

MISCEI.LANEOUS VETERINARY FORMULE . . . . . . 3OS

VETERINARY SURGEONS ACT . • . . . . . . . 325

IHE TITLE 'VETERINARY CHEMIST' • • • • • • . 33I

THE CONTAgIOUS Diseases (ANIMALS) ACTS. . . . $\quad 332$

SALE OF HORSES • . . . . . . . . . . . 334

VETERINARY CURRICULUM . . • • • • • • 335

Methylated Veterisary PREPARAtions • • • • • 336

ADMINISTRATION OF POISONS TO HORSES • • . . . 3.37

INDEX . . . . . . . . . . . 338 



\section{VETERINARY COUNTER PRACTICE}

\section{MEDICAL AND SURGICAL TREATMENT OF DOMESTIC ANIMALS}

Horses. - Medicines are usually administered to horses in the form of balls or draughts, sometimes as powders mixed with the food. Substances mixed with the food must, of course, have no very disagreeable taste, nor any marked odour, save some few regetable aromatics, as coriander, caraway, and anise, which are frequent ingredients of tonic and stomachic preparations. Bitter agents are not so much objected to, and many horses will eat Epsom salts. All powders are better mixed with damp food, or given in a mash.

Draughts are a very common form, and, for some agents, indispensable. In cases accompanied by sore throat they should be used cautiously, as the animal, from haring the head elevated, is apt to cough, and so allow a portion of the draught to pass into the windpipe-not a fatal accident, but one tending to aggravate the case, or even produce fresh complications.

In sore throats it is advisable to substitute electuaries, made either with honey or treacle, and merely smeared on the tongue, or put into a piece of muslin and placed as a bit in the mouth.

Horses can retain fluids in the mouth a long time without swallowing, and in this way sometimes reject medicine.

Balls are by far the handiest form of preparation, but only a small proportion of stablemen are able to administer thema fact overlooked, apparently, by those responsible for County 
Council lectures on veterinary topics, else instruction of a practical nature would be afforded in place of learned disquisitions on the origin of species and descent of the horse from the orohippus, the anchitherium, and the hipparion gracile, described by paleontologists. In the form of a ball there is a greater certainty of the animal getting the whole dose, as medicines mixed with the food may be left untouched, and, commonly, a good deal of a draught is wasted in the giving. Balls should be cylinder-shaped, not more than I inch in diameter, about $2 \frac{1}{2}$ inches in length, and about I Oz. in weight, although these dimensions may be safely exceeded in the case of large animals of the draught breeds.

They should never be used in cases of sore throat, as they irritate the part, and may be coughed back into the nose, from which cavity their removal is extremely difficult. It is thought by some people that medicines act quicker in the form of the draught than as balls. Numerous experiments upon horses show that nearly all balls are thoroughly dissolved in the stomach in less than half an hour. We may, then, when practicable, give the preference to balls as the best ordinary form of preparation for horses. Drenches, however, are to be given when the medicine is liquid, when the dose is large, and when very speedy action is desired.

It is generally believed that horses cannot romit, because very few people have seen them do so. In cases of ruptured stomach and diaphragm, emesis may be almost regarded as diagnostic. In stricture of the assophagus also there is return of the food, which more often than not comes through the nostrils (vii the posterior nares). 'The green colouring of nasal discharges, when horses at grass are suffering from catarrh, is due to some masticated food being coughed, or otherwise thrown into the nasal chamber, and must not be mistaken for glanders or other specific disease in which coloration of the mucus is a symptom.

Iimetics are not intentionally given to horses, but nauseants are prescribed as febrifuges, and their action is cicmonstrated by the paticut 'turning up his nose' (elevating the upper lip), 
at the same time raising the head, with a well-marked expression of disgust. Though it is well nigh impossible to excite romiting in the horse, it is very casy to induce nausea, the smell of a dirty bucket or stale mash in the manger being generally enough to cause it.

Medicines are also administered, though less commonly than in the forms already mentioned, by the rectum, as enemas or clysters; and under the skin by the hypodermic syringe.

Cattle differ from horses, principally in having four stomachs, and possessing the power to ruminate. By far the best form of administering medicine to them is the draught, as they are easily held, and have not, like the horse, the power of retaining fluids in the mouth for any length of time without swallowing. Balls are not very much used for cattle, as it has been thought that they remain undigested in the first stomach (rumen); but later experience does not confirm this, and many modern practitioners administer balls, especially of the tonic and cordial class, where a purgative action is not sought.

Probably, some portion of all fluids imbibed by cattle passes into the rumen also; for this reason, then, it is best always to give a considerable quantity of fluid with a draught. Twelve ounces sulphate of magnesia in 4 quarts of water is as active as $16 \mathrm{oz}$. in half the fluid.

Sheep may have medicines given to them in the same form as cattle, the dose, however, being only about one-fourth.

Pigs should have their medicines mixed with the food. When this fails - as, for instance, in cases of total loss of appetite-the draught is used. Great care must be taken not to pour fluids into these animals whilst they are squealing, as they are very easily suffocated.

The proper way to secure a pig, in order to drench him, is to introduce a running noose into the mouth, with the iooj, uppermost, so that he will draw it tight over the face when attempting to escape. The tushes prevent the cord from 
coming away if it has been properly done. With this he can be pulled to a post upon which a half hitch is made (see figs. I and 2 ). With such a point d'appui the necessity of a cord round his neck is removed, and greater freedom permitted when presently the draught gets into his gullet. A man can bestride him when so secured and hold him by the ears, while another pours the medicine carefully into his cheek, avoiding his tceth, which are very apt to crush a bottle and injure the patient. The time-honoured implement in pig drenching is a stout old boot

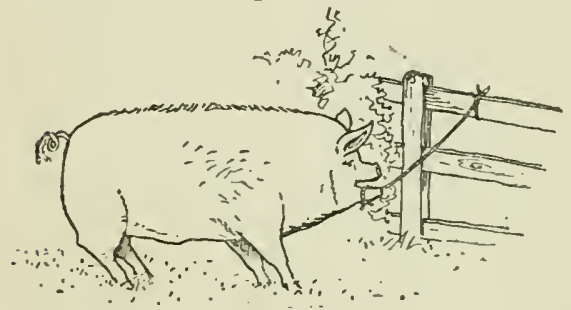

FIG. I.

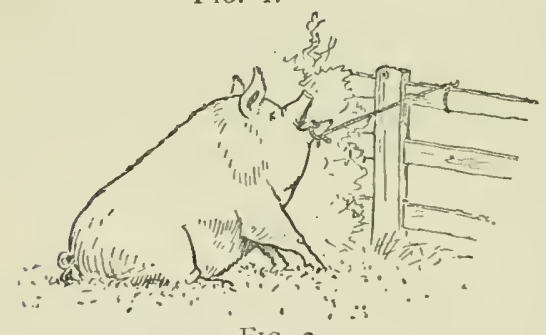

FIG, 2.

DRE.YCIIING A IIG. with the toe cut off ; it is a safe funisel which the angry patient 'champs' while the medicine is running through it. Some folk prefer to secure the pig by the lower jaw, as in the accompanying illustrations.

Dogs take medicines easily in the form of pills or draughts. When the appetite is good, substances may be mixed with the food, or, if nearly tasteless, sprinkled on a piece of meat. Pills, too, may often be kept d wrn by giving the dog a small piece of meat after them.

l'owders are easily administered by simply throwing upon the tongue, and, unless rery bulky, will be involuntarily sivallowed with the saliva. Most caninc practitioners cor:sider the powder the easiest means of giving medicines to dogs. l'ills should he made large, eren when intended for small dngs, as they are much casier to hold while pushing them over the back of the tonguc. 'There is not the least 
danger of the bolus going the wrong way, and it should be remembered that the gullet is so large in the dog that he can swallow larger bodies than can be passed through the small intestines subsequently, if they happen to be insoluble.

It must be remembered that medicines do not act with the same power on any animal if given on a full stomach. According to the circumstances, then, either increase the dose a little, or order it not to be given immediately after a meal.

Temperature.-The modern school of veterinarians have a great advantage over the old farriers by the introduc. tion of the clinical thermometer, whose readings in conjunction with other symptoms afford the most valuable diagnostic aid. IThen a practitioner had to judge a patient's temperature by putting his hand in the mouth, he had need have a well-trained hand indeed if after a long cold drive or upon a hot summer day he could detect an alteration of two or three degrees-not much to feel with the hand, but making all the difference to the patient's prospects. Thermometers are now so cheap and familiar that we need not describe them.

It was stated in former editions of this work that the temperature was not increased by a gallop, only the extremities and surface indicating a higher temperature by reason of the greater and more rapid distribution of the blood. The inference, for such it was, was drawn from experiments on horses galloped for wind-testing purposes in a short straw ride at the Royal Veterinary College, where the description accurately applied when the thermometer was employed; but since that time Professor Hobday, Mr. Willis, and other eminent reterinary surgeons have taken the temperature of large studs of omnibus-horses immediately after severe exertion, and found a considerable proportion with an elevation of several degrees, without any departure from health. Such high temperatures would prove deceptive were it not added that they quickly fall as the horse literally cools down.

In order to ascertain the temperature, the thermometer is usually inserted into the anus of the male or vaging of the 
female animal, as the mouth is obviously the least safe place for a glass instrument, and motives of delicacy do not preclude the veterinarian from using the thermometer in the way described except when some unusually obtuse lady cannot be induced to lose sight of her lap-dog when such happens to be the patient under examination.

In ruminants the temperature varies in health, seldom falling below $100^{\circ} \mathrm{F}$, and more often ranging up to ror ${ }^{\circ} \mathrm{F}$. Milking cows average about $102^{\circ} \mathrm{F}$, and may be often observed to be $103^{\circ} \mathrm{F}$. without any apparent departure from health. These figures are approximately correct, and are taken from a daily reading of thirty-five cows and fifty steers, heifers, and calves.

An increase of one or two degrees may or may not indicate a serious disease : $104^{\circ} \mathrm{F}$. or $105^{\circ} \mathrm{F}$. with a cough should put the veterinarian on his guard, and no harm can be done by isolating such a case until it has been decided whether or not the elevation of temperature is merely gastric derangement or indicative of specific diseases, such as pleuro-pneumonia or anthrax.

The Pulse of a horse is usually taken at the angle of the lower jaw, as there the submaxillary artery passes close to the bone and is easily compressed with the finger, giving one a sense of its volume and character as well as number. In a heavy horse the pulse should beat about thirty times per minute, and in a blood horse forty or even more. Pregnant mares have a quicker pulse, and of course exercise alters it according to the pace and conditions. In the above statement we are supposing a healthy horse standing still in the stable and free from either excitement or fear. Some horses are so nervous that the pulse is accelerated the moment a stranger walks up to them, and the examiner will be deceived unless he wait a minute or two till the creature's alarm has abated. Character is more than mere number, and nothing but experience can teach the importance to be attached to a full, round, a hard, a soft, a wiry, or an intermittent pulse. It may be stated broadly that a quick pulse indicates ferer, and an abnor- 
mally slow one suspended power, as in staggers (sleepy), or indicative of debility if it is weak as well as slow. Pulse and temperature and respiration need be considered together to be any accurate guide.

The pulse of the ox is felt at the radial artery under the fore-arm, at the back of the fetlock, or at the caudal arteries under the tail, at that portion where it is hairless. The last named is the most convenient, unless the animal happens to be vicious.

In dogs and eats it can be readily felt inside the thigh (the femoral artery), or taken at the heart, but they are such excitable creatures that too much reliance must not be placed on the pulse.

The following table indicates the normal pulse, temperature, and respirations of the various animals :-

\begin{tabular}{|c|c|c|c|c|c|c|c|}
\hline \multicolumn{5}{|c|}{ Animal } & Pulse & Temperature & Respiration \\
\hline Horse & . & - & - & - & $35-45$ & $100 \cdot 2^{\circ} \mathrm{F}$ & $8-12$ \\
\hline Cattle & . & . & . & . & $40-60$ & $102^{\circ} \mathrm{F}$. & $12-16$ \\
\hline Sheep & . & - & . & . & $60-S o$ & $103^{\circ} \mathrm{F}$ & $20-30$ \\
\hline Pig. & . & . & . & - & $55-75$ & $102^{\circ} \mathrm{F}$ & $2 \cap-30$ \\
\hline Dos & . & - & - & . & $70-90$ & IOI $5^{\circ} \mathrm{F}$ & $15-25$ \\
\hline
\end{tabular}

Setons and Rowels are old-fashioned remedies for producing counter-irritation, and are still largely used in some districts in the belief that quarter-ill, or blackleg, is thereby prevented.

A seton is a piece of tape, or string, or horsehair woven into a cord and passed through the skin by means of a large seton-needle, purposely designed to carry stout material, and having a very sharp point, so that one effort will make it go through the skin and avoid unnecessary pain. It is then dressed with some irritating material, as turpentine or liniment 
of ammonia, and knotted or tied to prevent its coming out. Large knots are preferable to a loop, as animals sometimes hang up in a tied seton. The dewlap is the part chosen for setons as a preventive for anthrax, but the idea is pretty well exploded and is fast falling into disuse.

Rowels are circular discs of leather introduced under the skin and productive of suppuration, as is the case with setons; they should be moved daily to keep up the issue. In some old pharmacies are to be found 'issue peas': these implements of torture used to be inserted in the necks of epileptic persons. The idea is the same whether for man or animals, namely, to set up an artificial irritation near the seat of disease or to give an issue to the ill humours with which men and animals were commonly supposed to be possessed in the days when spring and autumn bleedings and purgings were consented to by rational beings and endured by animals who had no choice.

Bleeding is an operation occasionally recommended in these pages, and although it is a practice which has fallen, to a great extent, into desuetude, there are urgent occasions for it ; the too free use of the fleam and the blood-stick last century has produced a revulsion, and it may be that the benefit of blecding is sometimes forgotten now among a generation who, perhaps, never used a fleam.

Bleeding may be general or local.

When it is desired to abstract a considerable quantity of blood, as in brain inflammation or congestion of the lungs, the jugular reins are those selected for the purpose, as these have the adrantage of being large and near the surface, necessitating only a small amount of local injury. Expert veterinary surgeons arrest the downward course of the blood in the vein by pressing the thumb or two fingers in the channel of the neck until the vein above the seat of pressure fills up, and feels hard and distended with blood, and then with a lancet in the other hand ruickly puncture the vessel, which immediately pours out a continnous stream of dark-coloured (renous) blood so long as the pressure below is continued, It cannot be expected of 
those who are not frequently called upon to blecd that they should do it in this manner, and a safer plan for them is to draw a cord tight round the neck, rather more than half-way down, and loop it so that it will press the channel of the neck and not come undone. A wine cork under the cord is found to facilitate matters very often. A little time should be allowed for the rein to fill up, and no attempt should be made until it looks like a rope under the skin ; it may then be opened by a lancet or struck with a fleam and blood-stick. The lancet has now almost entirely supcrseded the fleam; the latter was thought by some practitioners to be safer because it could only be made to go in a certain depth ; but, as a matter of fact, there is very much more danger from the blow with the stick than there is from going too deep with a lancet. Getting into the vein at all is the chief difficulty besetting amateurs, and the danger of wounding the carotid artery is more imaginary than real. So long as the bleeding is to continue, the pressure below the orifice must be maintained. A measure of some sort should be used to catch the blood, as a very little blood on a floor makes a great show, and one may be easily deceived as to the quantity abstracted.

When sufficient blood has been taken, the pressure of cord or fingers is removed, and very little difficulty remains in arresting it. The common and not very objectionable method is to take the lips of the wound between the forefinger and thumb of the left hand and run a pin through both lips ; from above downwards affords the greatest advantage to the operator, and the skins of some horses and cattle being very thick a good deal of finger force is necessary. The pin is then secured by winding a piece of tow round the ends like a figure of eight ; or if tow, thread, or the ravelled end of a sack or rope cannot be found, then a few hairs from the horse's tail or mane will answer the purpose. 'Tow we consider the most suitable, and advise a little ol. carbol. ( 1 in 20 or 30 ) to be applied at the same time. The pin should be allowed to remain for twenty-four hours, and the horse-if it be a horse-tied up so that he cannot rub it. Bullocks do not, as a rule, try to interfere with the pin. To rẹmove it, the hend end 
has only to be pulled steadily, leaving the tow adhering by a clot generally, which is best let alone.

Inflammation of the vein seldom follows, and when it does the best plan is to blister it down its course, as, failing to do its work inmediately, it is generally lost and becomes a fibrouslooking cord. Theoretically this seems a great objection to an animal, and writers have averred that horses so affected are liable to brain troubles if turned out to grass, \&c. Practically there is not much to fear from a lost vein, or even a lost artery, as collateral circulation is quickly established.

Bleeding from the jugular vein is usually practised on horses and cattle only, but there are kennelmen who bleed hounds in this manner, and with a boldness calculated to make an anatomist shudder.

Sheep are sometimes bled, but they are very bad subjects for blood-letting, and the custom of slitting an ear, the palate, or the eye-vein is perhaps better than attempting any larger vessels which might cause death.

Pigs are awkward subjects to bleed, and the ear is generally the part selected, the method the same as in the sheep.

Dogs may be bled from the jugulars, as preriously mentioned, but it is very rarely necessary to bleed them at all. The car should on no account be cut, as it is often most troublesome to heal, and the animal strikes it with his leg, and continually shakes his head.

Local bleeding is adopted to relieve particular parts, as at the toe, to relieve laminitis in the horse ; an operation now but rarely performed, as it is found that aloes and its preparations given internally do much more to reduce the congestion of the foot than blood-letting. More benefit is derived by poulticing and wet swabs in the case of inflamed feet : the expansion giving more room for the congested tissues to swell than the temporary cxtraction of blood, which is followed by increased inflammatory action at the seat of puncture. Blceding from the saphena vein is occasionally adopted in cases of swollen hind legs; the vein crosses the hock from behind forwards, and the part selected to bleed from is on the inside of the lower portion of 
the thigh, where the vein passes along the surface of the bone (tibia); the method is the same as for bleeding from the jugulars.

The eye-rein, which is seen standing out prominently on the side of the face, is occasionally opened with a view to relieve inflammation of the eye.

The palate of the horse, behind the incisor teeth, is the part selected for bleeding by stablemen, and in the case of a horse attacked with megrims on the road it is a good 'tip'; with no better instrument than a pocket-knife a cut may be made, and a few ounces of blood let out will more quickly restore the animal than anything else. Care should be taken, however, that the cut be not made beyond the corner incisors, as the palatine artery is apt to be injured, and bleeding from this is sometimes difficult to arrest.

Anæsthetics for Animals.-There is an increasing disposition on the part of owners of animals to have them anæesthetised as a preliminary to all serious operations.

For horses and cattle pure chloroform is to be preferred, and is practically free from danger. Dogs and cats are bad subjects for total anæsthesia, and the A.E.C. mixture (alcohol I vol., ether 3 vols., and chloroform 2 vols.), so much used in human practice, is by some chosen in preference to ether alone, which for dogs is safest, but in the case of cats too exciting. For the latter a mixture of 3 parts of chloroform and $\mathbf{I}$ of ether has been found to answer best.

Horses are commonly cast with hobbles and ropes (see fig. 3) before administering chloroform, as they fall quite as awkwardly and with as much risk to the spine if compelled to inspire chloroform from a muzzle while standing. There are a variety of patent muzzles for the purpose, but nothing more is needed than a sponge saturated first, and then the superfluous chloroform squeezed out, and introduced into one nostril, previously anointed with vaseline or lard. By depressing the wing of the opposite nostril during inspiration, the animal is compelled to inspire through the sponge, while the breath can 
be allowed to expire through the unoccupied nostril by releasing the fingers which held it during inspiration. The degree of anæsthesia produced is measured by the insensibility of the eye $t_{0}$ external influences, and by the shallowness of the respiration as shown by the flanks. The pulse can be disregarded as affording no indication of the animal's rallying power, but the anæsthetic should be temporarily suspended if the movements of the flank appear to be at a standstill. As a matter of fact, it is found to be very difficult indeed to kill a horse with chloro.

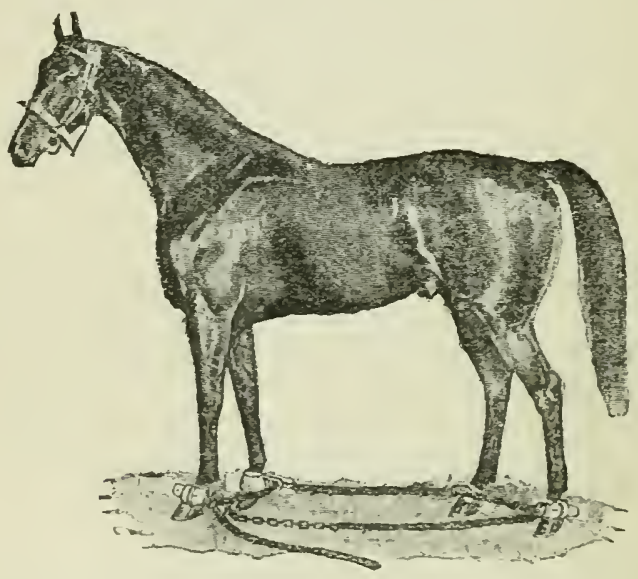

FIG. 3. - HORSE FITTED WITII HOBBLES (MADE BY MR, HUISII, S FISHER STREET, W.C.) REALY TO BE CAST FOR ANESTIETISING.

form when some merciful client wishes an old pet so disposed of.

$\Lambda$ cardboard cone containing a sponge, upon which the chosen anæsthetic has been poured, answers for dogs-presum. ing, of course, that the mouth has been already secured by a broad ribbon or soft cord to prevent biting, and that a capable assistant is available to forcibly control the patient until insensibility is cnsured.

The quantity of chloroform required to produce anas. 
thesia is so variable in different individuals that no purpose is served by naming any particular measure as likely to produce the desired effect; better is it to be provided with a surplus, and to observe the rules laid down as to failing respiration above indicated.

Professor Hobday has invented an apparatus for small animals which reduces risk to a minimum. It is on the principle of drop-by-drop administration in conjunction with atmo. spheric air.

Killing Dogs and Cats.-There are few chemists who are not called upon at some time or other to make an end of dogs and cats which have grown old and morose, dirty or dan. gerous, or in other ways offended against the unwritten code of laws which governs the conduct of domestic pets. If we recog. nise as part of our legitimate business the putting-out of exis. tence of animals of the class above referred to, the aim should be to do it in a manner at once merciful, rapid, and safe to the operator.

The following practical hints are offered for the benefit of those chemists who do not consider it derogatory to undertake work of this kind: Firstly, in the case of dogs, unless in extremis and incapable of resistance, it should be a stipulation that a collar and chain be attached to the animal, even though brought in a basket. It is better for sereral reasons that the owner or his representative should not be present at the death.

A muzzle should be placed on the dog by the owner either before he is brought to the shop or at the time; it is not fair or reasonable to expect a perfect stranger to do this on the first (and last) occasion he sees the dog. The chemist should not neglect to see for himself that the collar is buckled tightly enough, so that the dog cannot slip it in a moment of panic.

A cat should be brought to the place of execution in a basket from which she ean see, though she may not be seen. She feels safe in a crowd of dogs, so long as she is behind a wicker wall, and it is always panic that one has to fear in cats. A cat should not be rudely dragged forth, but allowed a little 
time to settle down after her journey. Then she should be addressed from the outside of the basket in tones of sympathy, when she will usually allow herself to be gently lifted out by the 'scruff' of the neck, or may be poisoned in the basket if soiling the latter is of no consequence.

If a cat is brought loose or has to be put into a basket, box, cr other lethal chamber, it should be remembered that pussy will go into a basket quite readily if put in hind-quarters first, but will almost surely resist if asked to go in head first.

The next thing is, What agent should be used? The use of hydrocyanic acid is sanctioned by long custom, but it is not a merciful poison. Chloroform is better, and a drachm or two of chloroform is not very costly. It is certainly a longer if not a more troublesome process, but there is the satisfaction of having killed the animal without pain.

If it is decided to use hydrocyanic acid, the syringe is most easily inserted in the rectum, hardly any objection being offered. A full drachm for a cat or small dog is the quantity, but twice as much is required for a large animal. If it is preferred to administer the poison by the mouth, there is no necessity for forcing the mouth open ; the lips either of dog or cat can be parted, though the muzzle is retained in position, and it is only required to pour or squirt the fluid into the cheek. The animal must die if this is donc, and the cnd will come quite as soon as though the jaws had been clumsily prised open and the acid poured into the mouth (and more or less spluttered out).

There is no art in chloroforming to death sare that of getting the victim into a close chamber, putting in the chloroform on a sfonge or two, and shutting the animal in. The instinct of seif-preservation is as strong in domestic pets as in wild animals, and, suspicion once aroused, they will often exercise marrellous cunning, and keep the nose close to a tiny crevice without an attempt at a struggle, after once realising the situation. A rug or towel should be covered over the box or bag, as few are free from leakages, and the end will sooner be attained and with less expenditure of chloroform, if these pre- 
cautions are taken. The danger lies in 'letting the cat out of the bag ' too soon, as it is difficult to get an animal to return to the lethal chamber after a first experience.

The chloroform death is the most humane method, and one which will earn the chemist respect, whilst entitling him to a good fee, from his customer. 


\section{VETERINARY MEDICINES}

[Nunerous formule are given in the subsequent chapters of this book in which diseases are specially treated. In this section are introduced the medicines generally suitable for a chemist and druggist's stock.]

Physic-mass.-'Physic,' in the language of the stable, belongs only to balls, whose chief constituent is aloes, and whose mission is to purge. The collective experience of the best veterinary surgeons and horseowners goes to prove that, of all the varieties, Barbadoes aloes is the best for horses, more certain in effect, less nauseating, not disposed to gripe or leave a horse debilitated and without appetite.

It is a matter of common knowledge that veterinary surgeons charge is. $6 d$. for a physic-ball which a druggist can very well supply for half that sum, and many do sell them at the rate of Id. per drachm. The ball supplied by the vet. is, however, better worth the additional money, because he adds the useful advice, 'Prepare the horse before you give it'; and if he finds the purchaser does not know the meaning of the words he proceeds to explain that to obtain the best results from alcetic (physic) balls the animal should be kept on bran mashes for twelve to twenty hours, and not allowed hay or any other dry food, but that he should have plenty of water allowed him, with the chill taken off by adding a litle hot water to it, according to the temperature of the air \&c., walking exercise being also enjoined before the medicine begins to purge but desisted from immediately the bowels respond. In this consists the chicf reason why "veterinary surgeons' physic-balls often give hetter satisfaction than druggists'.

The practice of giving a dose of physic in the spring and autumn still obtains in many districts, and, we think, with 
decided advantage to the equine population of those parts, since long experience shows that fewer cases of impaction and bowel troubles occur where such an occassional purgative is given; this particularly applies to horses habitually fed upon dry corn and chaff, hay and straw, \&c., and it requires but a moment's reflection to convince the practical horseman that an animal intended by Nature to live on grass should sometimes require artificial assistance when habitually subjected to artificial conditions of life and diet.

Physic balls made with soft soap are apt to become very hard, and it is open to doubt if the efficacy of aloes is not impaired by it after a while. Professor Tuson's formula for a mass will be found most convenient, though we have not met with any formula entirely free from objection.

\section{Prefessor Tuson's Formula.}

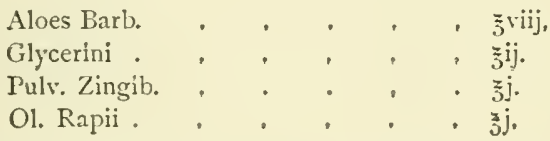

[3ij. of glycerin and $3^{\mathrm{v} j}$, of oil we have found a better excipient for keeping.]

Melt together in a water-bath and thoroughly incorporate by stirring.

To make physic mass on the large scale the aloes is melted in a steam-heated pan, or, if this is not available, in a waterbath. When melted, if poured on a slab and worked up with the glycerin and ginger, a smaller proportion of glycerin than that indicated, $I$ in 6 or 8 , will suffice, and a mass will be produced which will keep plastic for any length of time, and which is always readily soluble. Masses or balls which get hard from age or other causes are always erratic in action, and sometimes cause irritation to the stomach.

The following formula for a mass which is plastic, ductile, 
and soluble, was published some years ago by Messrs. Elliman $\&$ Co., the makers of the well-known embrocation :-

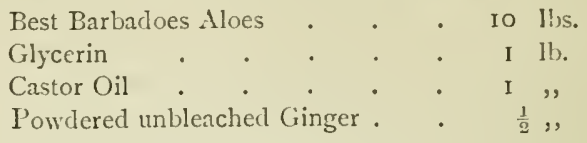

Dissolve the aloes in the glycerin by means of a waterbath, then add the castor oil, and lastly stir in the ginger, previously sifted through a coarse sieve. Balls made from this mass will not retain their shape, but may be wrapped in waxed paper and put up in cardboard boxes similar to those used for marking-ink.

The dose of aloes for an ordinary carriage-horse is $5 \mathrm{v}$. or $5 \mathrm{vj}$, and for a heavy cart-horse $5 \mathrm{vj}$. or $5 \mathrm{vij}$. in the southern part of Britain, but in Scotland a full drachm or drachm and a half more is not found too much. For every drachm of aloes take one drachm and a quarter of the above mass.

The combination of ginger with the aloetic mass, as in the above formula, is very usual, but this addition is of but little importance. The addition of gentian increases the action of aloes, as does a previous course of iron. Aloin is sometimes given to high-bred and delicate horses in the proportion of about one-quarter to one-half of one dose of aloes.

A very clean and efficacious mass is made by adding carbonate of potash. Care, however, must be taken to stir the ingredients frequently when melting, as a certain amount of effervescence takes place and a quantity may be lost.

\begin{tabular}{|c|c|c|c|c|c|}
\hline Aloes & . & & & & IS $n z$ \\
\hline l'ulv. Kingils. & . & & & • & 2 \\
\hline Ol. Lini . & . & . & . & . & $1 \stackrel{1}{2}$ \\
\hline J'otass. Carb. & . & . & . & . & 2 \\
\hline Aquix $\quad$. & e & • & • & , & 2.? \\
\hline
\end{tabular}

Melt together in a water-bath. Calculate the dose as required. Alocs forms two thirds of the mass.

About eighteen hours is the time a physic ball takes to 
act, and exercise should be enjoined, but not to continue moving the animal when purging has commenced; nor should he be put to work until the 'physic is set' or the dung normal in consistence, and a feed or two of corn has been consumed.

Condition Balls. - 'Condition' is a term used variously; but with horsemen it means one of two things-either 'hard' condition or 'show' condition ; and the individual who would gain a name for condition powders should clearly understand which condition it is he proposes to assist in bringing about.

The condition of the hunter, or even of the fast hack or harness horse, is 'hard'-that is to say, the muscles are in the condition of a prize-fighter, who 'trains' off his fat and derelops his fibres to the greatest degree of strength for muscular exertion and heart and lung power ; the condition of which latter organs more often decides a race or a fight than the muscles of the limbs, as it is upon the respiration and circulation that endurance depends.

To condition a horse for racing, hunting, or trotting, first give him a dose of aloes or physic proportionate to his size and breeding; for a medium-sized animal of about fifteen hands the average dose of aloes is 5 or 6 drachms, and rather more or less according to size; say, 4 drachms to a pony and 8 to a cart-horse ; the physic to be followed by balls or powders according to breed. Of actual training we do not propose to speak here, but merely to indicate the most suitable medicaments. For 'hard' condition, then, we would say, give those remedies which will aid assimilation of food, help the skin to act and cast the old coat, enabling the superficial nerves to receive that stimulus from the fresh air that a horse in his natural grease can never know, and, to use the words of Professor Simonds, 'call upon those emunctories the kidneys.' The use of diuretics is very common, and too frequently abused; but no condition powder or ball will do its work thoroughly unless it assists the kidneys to get rid of the vast amount of effete material which is the neccssary result of so much waste of muscle and other tissues in large animals. 
The following is a formula for a ball which closely resembles one of much renown :-

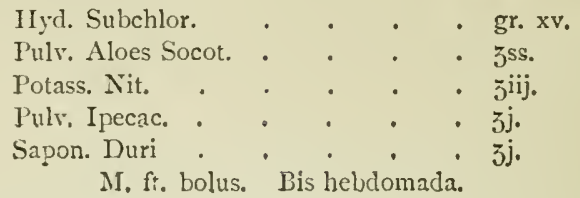

The administration of these balls should not be continued for more than a month, as the horse is very susceptible to liver stimulants. He will often vastly benefit by the above, but if the treatment be too long continued he will go down again and lose his bloom. The following is also a good ball with a very wide sale, and may be given to a 'bad thriver,' or horse that habitually requires assistance to prevent him having blotches and swellings, and also in cases of loss of appetite :-

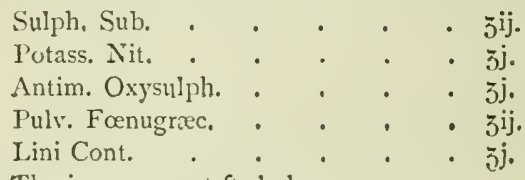

Theriacæ, q.s. ut ft. bol.

Omni alt. nocte sumend.

Show Condition.-To bring about the best results artificial feeding and the use of various cordials are resorted to. Everyone admires, while condemning, the cart-horse parade and the fat ponies that are shown for prizes, and as long as the public will give premiums for horses unnaturally fat there will be a demand for those agents which help the process. They are : Cordials, stimulants, carminatives, digestives, diuretics, and diaphoretics; but if there is sufficient time, it is always best to begin, as before, with a dose of 'physic' proportionate to the size of the animal. This is followed by a course of doctoring which is by no means to be invariably recommended. 'The basis of nearly all the advertised horse and cattle spices is salt, lentil-flour, and fenugreek [several recipes will be found on page $\left.31_{2}\right]$. But the effect of these 
condiments is greatly augmented by such agents as sulphur, antimony, nitre, Sc., which enable the skin and kidneys to get rid of superfluous material that is liable to cause eruptions or blotches just as the show-week is approaching. There is another agent that makes the coat shine with less trouble than elbow-grease and antimony, and that is acid. sulph. daily in the drinking-water. This is the remedy which cunning carters use, who would rather buy a penn'orth of oil of vitriol than give a fair amount of strapping to their team. For balls to help show condition the following cannot well be beaten:-

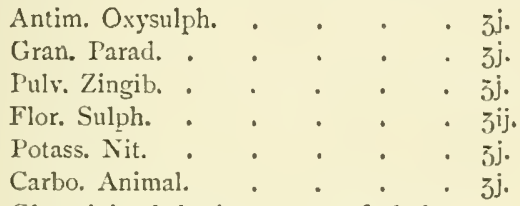

Glycerini vel theriacæ q.s. ut ft. bolus.

Bis hebdomada.

The following is also a good condition-ball :-

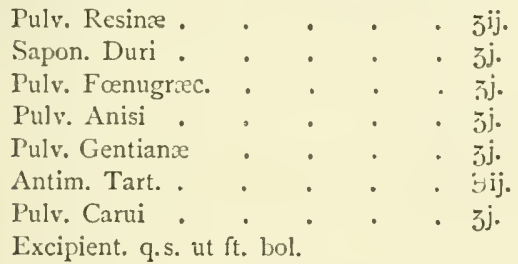

Omni nocte.

If there is an objection to antimony, give the following :-

Potass. Bicarb. • • • • $3^{i j .}$

Sodii Chlor. . . . . . $3^{\mathrm{ijj} .}$

Pulv. Zingib. . . . . . 3 ij.

Flor. Sulph. . . . . . jij.

Excipient. q.s, ut ft. bol.

Omni nocte.

It is hardly necessary to say that the sodii chloridum should be moderately dry, and the least amount of excipient used, or the balls will absorb moisture and fall. 
Condition Powders. - When the attendant is capable of giving balls they are always to be preferred to powders, because alterative doses of aloes and other nauseants can be given which the horse will not eat in a mash or with corn. With a ball the horse is sure to get the right dose or none, as he either swallows it or not; but in powders some part falls to the bottom of the manger and is not eaten, or a double dose may be licked up with a more than usually wet mash, and a measure is not likely to be used, the attendant's fist being substituted for it. But there will always be more buyers of powders than balls, and to suit them the chief ingredients of the recipes given above may be combined, omitting the important agent aloes, and without the soap, though some horses will eat aloes if properly powdered and flavoured with salt \&c.; but then some horses will eat dung and leather, and we must here prescribe for the average horse, who is very easily put off his feed by the smell of drugs, and will show his sense of nausea by 'turning up his nose' in the literal and not the metaphorical sense in which the term is often used, and from which action it is doubtless derived. The following is the next best thing to the ball first mentioned:-

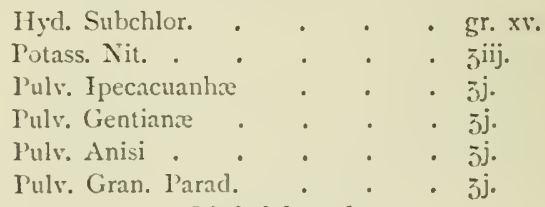

Bis helodomada.

This may, of course, be coloured with bol. armen. or curcuma, or other innocuous material.

The sccond ball, in powder form, is very palatable, on account of the fenugreek. If, however, a horse refuses it, add to each dose

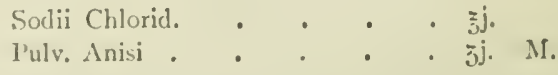

None of these agents are expensive, but for those who want a still cheaper horse-powder to sell, in tins of $\frac{1}{4} 1 \mathrm{lb}$., $\frac{1}{2} 1 \mathrm{lb}$., and 
I lb., there is nothing better than the old-fashioned mixture of equal parts of pot. nit., flor. sulph., and antim. nig. Half ounce doses of mag. sulph., sufficiently dried, powdered, and mixed with salt, flavoured with a drop or two of ol. anisi or ol. carui, make a good powder for giving bulk to the above, and are a good thing for swellings, as we shall presently show in dealing with alteratives, \&c. For a more tonic powder mix ferri sulph. 2, pulv. gentian. 2, and pot. nit. I, and give $\frac{1}{2}$ oz. for a dose.

Alteratives.-Alterative medicines are in constant demand, especially in the spring and early autumn, when the shedding of the coat produces a certain amount of debility or lassitude, or when from previous clipping or exposure the skin does not act freely. Indigestion or surfeit, causing eruptions, urticaria, or water-bumps, and a variety of skin affections too numerous to mention, are treated with alteratives, and more frequently than not with success, unless the irritation of the skin arises from parasites, such as mange, of which we treat later on.

The following balls and powders are intended as alteratives rather than diuretics, though a combination is frequently and advantageously made. When a horse is 'out of sorts,' or does not feed well, or passes his dung with difficulty, or the dung is a bad colour, and from these symptoms and his general behariour he appears to be out of health, but not sufficiently bad to send for a veterinary surgeon, he may be given a few of these balls or powders without having to knock off work-a most important consideration.

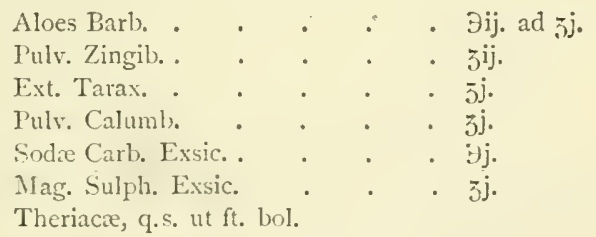

Capt. j. omni nocte.

This may be given as a powder by omitting the aloes and tarax., and substituting pulv. fonug. or sodii chlor.

Another good alterative where the lymphatics are concerned, 
corded veins or swollen glands (as distinguished from ordinary swollen legs), or where farcy is suspected, is the following :-

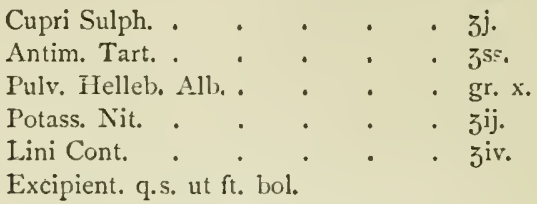

Omni nocte.

This can be given as a powder with damped food, as very few horses object to eat cupri sulph., especially if a little fonug., or anisi, or pulv. carui is added.

Diuretics are so well known that they require very little comment. They should not be too frequently resorted to, though they are of great benefit when judiciously administered. They excite absorption of fluid in the limbs, sheath, \&c., which should be assisted by exercise and diet. The following are valuable recipes:-

\section{I.}

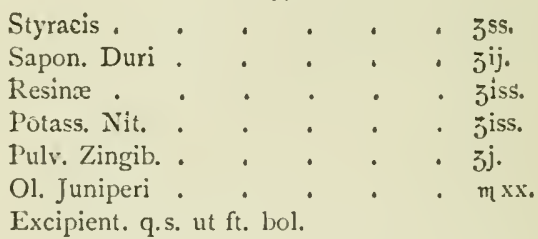

One occasionally.

II.

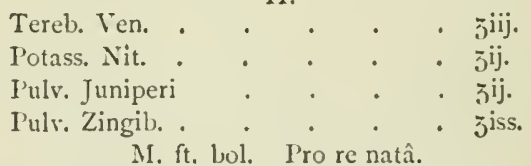

A simple and useful diuretic mass may be made from the following recipe :-

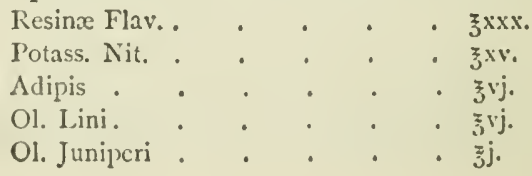


Melt the resin and add the oils and the lard. Next add the nitre and stir till nearly cold, then transfer the mass to a slab and work up with glycerin.

Cordial Balls. - These are given to horses which are faint and off their feed on returning from hunting, racing, or fast work-where, in fact, the muscular system is superior to the digestive, and the 'spirit carries them beyond their strength.' A cordial ball given at such times is of great value, restoring a flagging heart and circulation, which by the morning might result in congestion of the lungs. The following is an excellent cordial ball, but has the disadvantage of not keeping well. It is for such balls that gelatin capsules are suitable :-

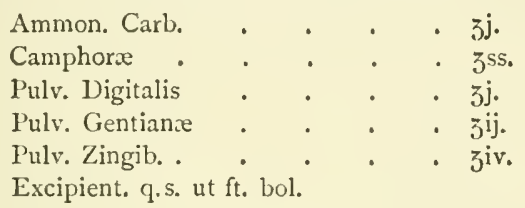

Two or three of these balls may be given in eight or ten hours, but should not be persisted in. The following is the cordial ball of the Veterinary Pharmacopœia :-

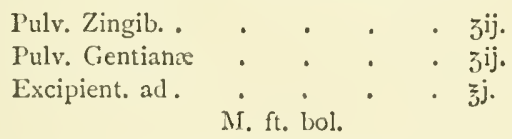

The following is much approved in hunting-stables, and can be well recommended :-

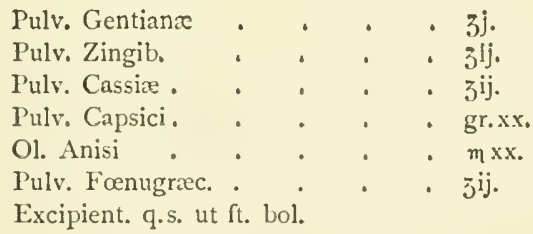

Clients should be advised to pull the ears, bandage the legs, clothe the body, and thus seek to obtain surface warmth. 
Fever Balls.-These are not so much in demand as formerly; when clinical thermometers were not in general use and the subject of ferer was less understood. The following is a well-tried ball, and may be given in undefined fever, with cold ears and legs and staring coat, without any special cause being ascertained :-

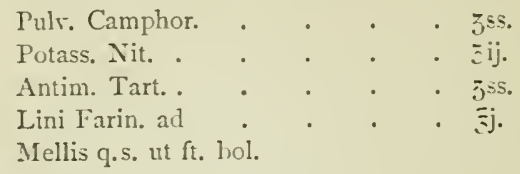

Not to be given oftener than twice a day, or continued for more than three or four days. For the tartar emetic nay be substituted calomel I 5 grains; opium I 5 grains.

The following is a useful ball where a stimulant is required as well as a febrifuge :-

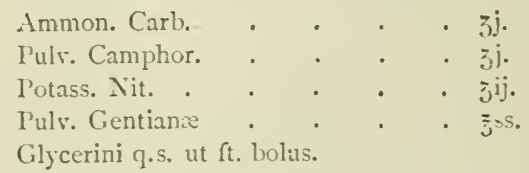

Sig. : One to be given night and morning.

Tonics. - Both the regetable and mineral worlds afford considerable latitude in the choice of tonics, though in vetcrinary practice only a few are commonly uscd. Selcction is guided by the cause of the debility. As a rule, when the reakness depends upon some blood-poison, as 'farcy;' or some excessive drain upon the system, as great suppuration, dropsy, \&c., mineral tonics are most useful; when it is the sequel of some acutc discase, as pleurisy, or accompanicd by an irritable stomach, regetable tonics are preferable. It is often desirable to combine tonics with other medicaments, as with stomachics or diuretics. As regards the form of administration, balls or powders are the most convenient. Sometimes draughts are necessary, as in the combination of stimulants, such as ether, with tinctures. 
A valuable stimulating tonic for cattle convalescent from any very exhaustive disease, pleuro-pneumonia (non-contagious), or during the later stages of red-water, is the following:-

$$
\begin{aligned}
& \text { Ether. Sulph.. • . . . 3ij. } \\
& \text { Tr. Zingib. • . . . . 3ij. } \\
& \text { Tr. Gentiane . . . . . 3ij. } \\
& \text { Misce pro dosis. }
\end{aligned}
$$

Four doses as above-one every six hours in a pint of gruel.

Of mineral tonics, iron, copper, and arsenic are the farourites. Of the preparations of iron, the sulphate is the only one commonly used for horses and cattle. It is generally given combined with some aromatic, as--

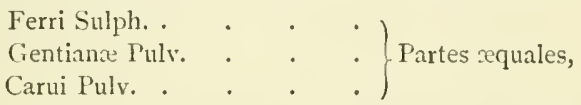

made into balls with treacle, or given in powder with the food. Coriander seeds or ginger may be sulsstituted for the caraway. Sulphate of copper is sometimes used in place of iron, and it seems of special value in cases of chronic nasal discharge. The ordinary dose of either sulphate of iron or copper for horse or ox is 2 drachms, but in the above cases can be doubled. As much as 5 drachms of sulphate of iron has been given daily for three weeks, but there is always a tendency to constipation with such doses, and the freces are blackened, owing to formation of iron sulphide in the bowels. To guard against constipation, small doses of linseed oil or the frequent admixture of bran or other laxative food, is recommended.

A good tonic ball may be made from the subjoined formula :-

$$
\begin{aligned}
& \text { Ferri Sulph. Exsic. • • • } 3 \text { ij. } \\
& \text { Potass. Carb. . . . . } \tilde{\jmath}^{\mathrm{ss}} \text {. } \\
& \text { Pulv. Nucis Tom. . . . . } \bar{j}^{\text {ss. }} \\
& \text { Acil. Arsen. . . . . . gr. ijss. } \\
& \text { Glucos, vel theriace q.s. ut ft. bol. }
\end{aligned}
$$

It is sometimes desirable to give iron in a less astringent form than the sulphate, but experience proves that with the 
water of crystallisation in it, it is more active and less irritating to the stomach than many of the more elegant preparations preferred in medical practice. The following formula for a solution (commonly known as 'dialysed iron'), contributed to The Chemist and Druggist some years ago, fulfils the re. quirements :-

Take of liquor ferri perchlor. fort. ${ }_{5}^{z} x x x$., dilute with 2 pints of water, and add ammonia solution in slight excess; allow to stand one hour, and wash the precipitate by decantation until the washings give no precipitate with nitrate of silver solution; throw the precipitated oxide of iron on a filter, and drain well. Now dilute $5 \mathrm{oz}$. more liquor ferri perchlor. fort. with I pint of water and dissolve in it the precipitate obtained above, and make the solution measure $\mathrm{I} 40 \mathrm{Oz}$. The result is a dark red-brown liquid, I oz. containing an equivalent of $3^{2}$ grains of ferric oxide. The dose for horses and cattle is I to 2 oz., diluted with water to ro oz.

An excellent tonic mixture is made by dissolving quinine in this solution, with the addition, if necessary, of a few drops of dilute hydrochloric acid.

Each ounce of the above solution contains the same weight of iron as $68 \frac{1}{2}$ grains of sulphate, but only $1 \pm \frac{1}{2}$ grains of hydrochloric acid, as compared with $23 \frac{1}{2}$ grains of sulphuric acid in the equivalent proportion of ferri sulph.

The tinct. ferri perchlor. and syrupus ferri iodidi are valuable preparations for dogs. The following is a good form for a dog recovering from pleurisy :-

$$
\begin{aligned}
& \text { Syrupi Ferri Iodidi • • • • } 3 \text { j. } \\
& \text { Infus. Gentianxe . . . . Jlij. } \\
& \text { Tr. Zingib. . . . 3iss. }
\end{aligned}
$$

M. fiat mist.

$\Lambda$ dessert-spoonful three times a day. The dose of tinct. ferri perchlor. is from ten to thirty drops, and it may be given with infusion of quassia.

Another valuable mineral tonic (for horses) is arsenic, sometimes given as a powder, incorporated with sugar of milk or 
carbonate of potash, or it may be made into powders, as follows :-

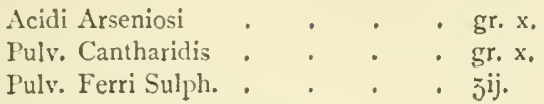

M. It. puls.

Once a day with the food.

Arsenic is specially useful in cases of broken wind, chronic cough, and in such skin diseases as do not depend upon parasites, but such powders as the above should only be supplied to responsible persons: carters and others are prone to increase the dose and continue to use 'coat-shiners' in lieu of ' elbow-grease.' It should also be known to readers that the employé who gives such drugs, without his master's knowledge, is liable to imprisonment for unlawfully administering drugs.

The best preparation of arsenic for dogs is Fowler's Solution, in doses of from 2 to $S$ minims. The following mixture is recommended :-

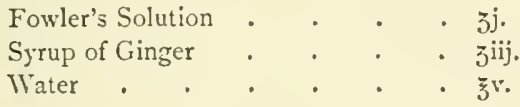

A tablespoonful thrice a day. Useful in skin diseases, and in chorea or other nervous affections following distemper.

Another mineral substance of great service is common salt, equal parts of salt and gentian forming one of the best of mild tonics. A tablespoonful twice a day for horse or ox.

Of vegetable tonics, cinchona probably deserves the first, and nux romica the last, place, but the latter is considered to be a valuable liver tonic and digestive stimulant: a good medium is the much-used gentian. A useful general tonic is the following :-

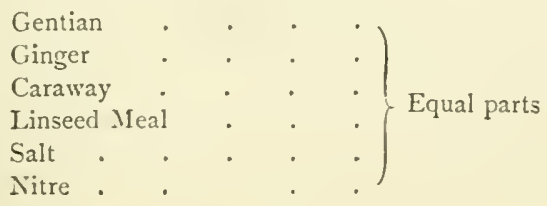


A more powerful tonic for horse or ox is-

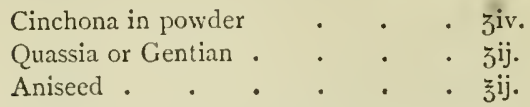

Can be given as a powder, or made into a ball with treacle. For a dog the following is recommended :-

$\begin{array}{lllll}\text { Pulv. Cinchonæe } & \cdot & \cdot & \cdot & \text { 3iv. } \\ \text { Ext. Gentianæe } & \cdot & \cdot & \text { jij. }\end{array}$

Make into forty pills, two to be given twice a day.

Quinine made into pills with glycerin of tragacanth is of equal value. Dose, I to 8 grains. For horses or oxen I to 2 drachm doses, and more, of quinine are sometimes prescribed, but it is doubtful whether it is so good as cinchona.

If a tonic is required after influenza or other chest affections the following may be used :-

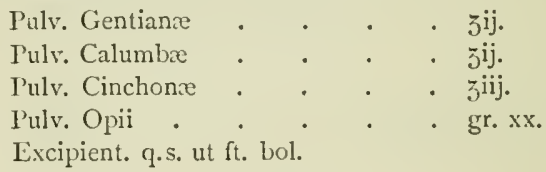

Bis die.

A little green food or bran mashes should be given with these, as small doses of opium tend to constipate, though acting as a tonic and stimulant. Major F. Smith, of the Army Veterinary School, and other prominent men hold that opium is better as a stimulant and tonic than as a sedative in large doses. The above can also be given as a powder, flavouring, if necessary, with salt, or foenugreek, or aniseed.

The above balls may also be made up with liq. strychninæ hydrochlor. $5 \mathrm{j}$. to each.

Nux romica and strychnine, as nerrine tonics, are sometimes given to dogs in the following doses:-

Nux Vomica .
Strychnine

Nux vomica and strychnine are, however, unsafe medicines for dogs, and chemists had better avoid them. 
Balls for Swollen Legs.-For a general ball for swelled legs, where there is not time or opportunity to go into the particulars of the case, the following can be given with safety, and repeated at comparatively short intervals :-

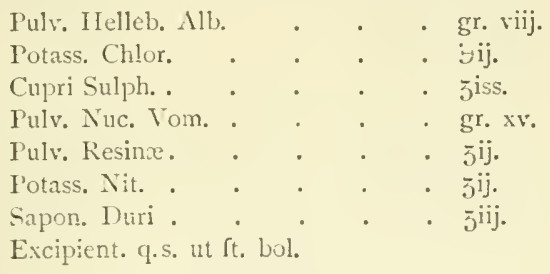

Cough Balls.-In the early part of the winter, when horses-are first clipped, they are apt to take cold and suffer from cough, which can be greatly relieved by a few balls or powders, and these should be ready to hand as the season comes round. Cough balls and powders should be of two kinds-those for temporary or acute cough, and those for chronic cough.

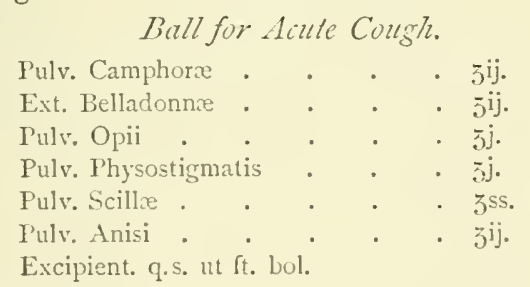

This ball may be given night and morning, and a little lin. saponis prescribed for the throat if a difficulty in swallowing is evinced.

Chronic cough and broken wind is a condition of many old horses, and it is surprising how horses so affected can be kept going by the aid of medicines, especially those containing tar and fats, though dieting is a very important factor, and should not be forgotten by the prescriber if he would get fair credit for his treatment. The food of such horses should be given damped, very little hay or bulky food, with a bed of moss litter 
or sawdust : that he will not eat. Linseed oil or linseed mashes or tea should also be given frequently.

\section{Chronic Cough Balls.}

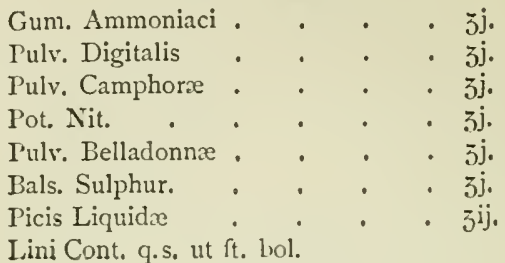

Omni nocte.

This is rather a messy ball, on account of the tar and balsam of sulphur, but is so good in its effects that the dispensing objection should be disregarded.

Another good ball may be made by mixing equal parts of melted and strained mutton fat with tar, using this mixture as an excipient, and working up into balls of nearly $2-0 z$. weight with the dry ingredients of the above ball.

If a powder is desired (it would not be so effectual), the above ingredients may be used with 2 or 3 drachms of pulv. glycyrrhizæ as a substitute for the camphor, tar, and bals. sulph.

A rery good powder for coughs is the following:-

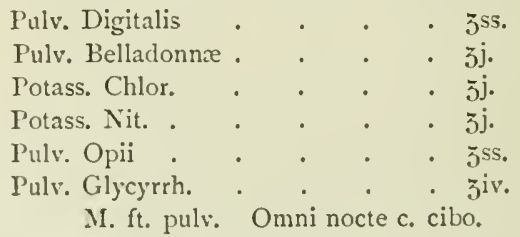

We add two farourite recipes for stock cough balls :-

Pulv. Ipecac. . • • • 3j.

Pulv. Scille . . . . j j.

Mcllis et Pulv. Glycyrrh. q.s. ut ft. bol.

Pulv. Ammoniaci • • . . jiv.

Pulv. Zingib. . . . . . jiss.

Pulv. Scillie . • • . • зj.

Mellis ct I'uls. Glycyrrh. q.s. ut ft, bol. 


\section{Cough Electuary for Horses.}

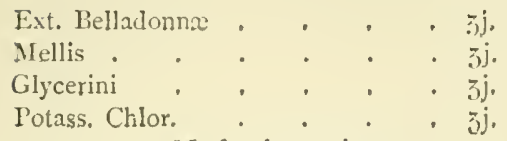

II. ft. electuarium.

To be given on a bone spatula by pulling out the tongue and smearing on the thick portion. It may be well to mention here that in nearly all coughs, save those chronic ones connected with digestive troubles, a stimulating liniment should be supplied, with advice to rub it well into the throat. Lin. ammon. or lin. camph. co. is suitable, and may be repeated until counter-irritation is proved by the lifting of the cuticle. The formula for a liniment given on page 35 is very popular among veterinary surgeons, masters of hounds and others with experience of the subject.

Gripe Medicines for Horses. - If colic arises from drinking well-water, and is but a simple spasm, it will generally pass off with a stimulant or the gripe remedies in common use, but a number of horses die annually for want of an aperient in the first gripe dose they get. This is so well known to practitioners that the majority of country vets. put a solution of aloes in their draughts, and give directions upon the label to give a pint or more of linseed oil with the draught. An effectual gripe draught for general use, without stopping to inquire into particulars, or without the advice of a practitioner, should contain an aperient, must be of some considerable bulk, and camnot be very cheap. The anodynes used in medicine for horses have undergone a considerable modification of late years, as experiments have proved that some of the old agents have not been correctly understood, and more direct sedatives have come into use. One of the most popular authors of thirty years ago speaks of opium as the "sheet-anchor of the veterinarian,' and it is doubtless a very valuable agent, but for controlling pain it is found that ext. cannabis ind. and chloral and 
croton chloral, where impaction exists, answer better. The following draught has been found to answer better than any other combination, but it has the objection of being rather expensive :-

Sol. Chloral Ifyd. (I gr. in I minim) zij.

Ext. Cannabis Ind. . . . 3 ij.

Ol. Terebinth. . . . $3 \mathrm{ij}$.

Spt. Ammon. Ar. . • . . $3 \mathrm{j}$.

Sol. Aloes $(\mathrm{I}$ in 4$)$. . .

Ol. Lini ad . . . . $3 v j$.

M. ft. haust.

To be given in from $\frac{1}{2}$ to $\mathbf{I} \frac{1}{2}$ pint of linseed oil.

This draught is an aperient, a diuretic, and an antispasmodic; it allays pain and checks inflammation, and if gripes arise from impaction it is doing valuable work while the veterinary surgeon is being sent for. Such a dose ought to he charged 2s. $6 d$. For a chcaper one the following may be supplied :-

Sol. Chloral Hydrat. ( 1 in $\mathbf{1}$ ). • zj.

Spt. Eth. Nit. . . . . zjjs.

Lals. Copaibre . . . . jiv.

Liq. Potasse . . . . . $\overline{5}^{\mathrm{sS}}$.

Ol. Terebinth. . . . . ङiv.

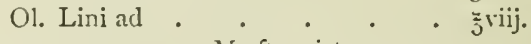

M. ft. mist.

A fourth part for a dose, in from $\frac{1}{2}$ to $\mathbf{1} \frac{1}{2}$ pint of linseed oil.

$\mathrm{Or}-$

Chloral Hydrat.

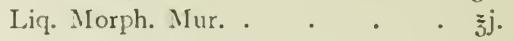

in half a pint of water, either plain or coloured. Often when all else fails to give relief the following may be recommended, especially if there is a suspicion of inflammatory conditions :-

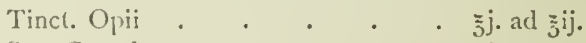

$$
\begin{aligned}
& \text { Spt. Camph. • . . . . } \\
& \text { Tinct. Aconit. B. I'. • • . Mxr. }
\end{aligned}
$$

diffused in a pint of cold gruel or old ale. 
$A$ good laxative colic drink for horses is-

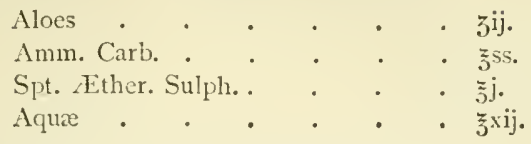

or one draught. It is an excellent remedy.

\section{Outward Applications for Horses.}

White Oils.

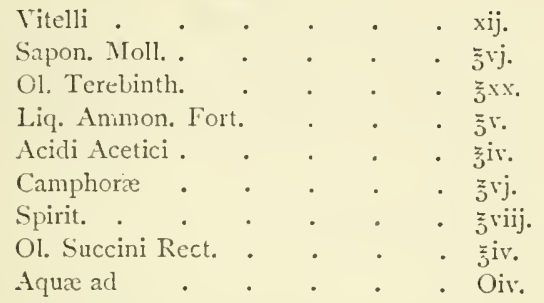

Rub the soap gradually with $\mathrm{I} \circ \mathrm{oz}$. of water to form a smooth jelly; add the spirit with the camphor dissolved in it; mix the turpentine and the oil of amber ; add gradually to the mixture, stirring assiduously the while, and aiding emulsification by the occasional addition of a little water. Then add the ammonia. Now transfer to a Winchester, add gradually the acetic acid diluted with a pint or more of water; add the eggs one by one, well shaking all the time, and finally make up to So oz. with water. [The liniment is better without the acid.]

For Sprains and Bruises, where the skin is not broken, we recommend:-

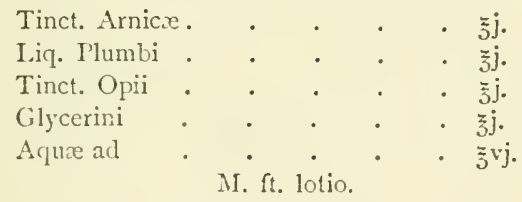

To be mixed with a gallon of rain or distilled water, and applied on wet bandages. (This is equally good for horned stock.) 
Saddle and Harness Galls.-A good and cheap lotion is the following:-

$$
\begin{aligned}
& \text { Acid. Sulph. . . . . . 5j. } \\
& \text { Alum. Exsic. . . . . . } 3 \mathrm{ij} \text {. } \\
& \text { Aqua ad . . . . Oss. } \\
& \text { II. ft. lotio, Sxpe utend. }
\end{aligned}
$$

Or-

Pot. Permang.

Alum. Pulv.

- gr. j.

- $\tilde{3}$.

in a pint of water, applied as a lotion. This should be made up as wanted, and not kept in stock.

The prescriber should always advise padding the collar or harness in such a manner as to aroid rubbing the scabs off, or remedies will be vain.

If an ointment is preferred-

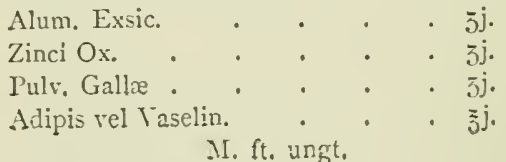

To be applied constantly.

Broken Knees.-Poultice for two days with linum cont., adding to each poultice-

$$
\begin{aligned}
& \text { Acid. Carbolic. . . . } m x \text {. } \\
& \text { Olei Olivie ad . . . . } 5 \mathrm{j} \text {. M. }
\end{aligned}
$$

This may with advantage be coloured with ol. viride or alkanct, or 'nosed' with a minim or two of ol. caryoph., which latter is a fashionable remedy for broken knees.

When proud flesh appears it is soon enough to use-

$$
\begin{aligned}
& \text { Zinci Chlor. . . . . . gr. x. } \\
& \text { Sacch. U'sli . . . . . q.s. } \\
& \text { Aqux ad . . . . . Oss. }
\end{aligned}
$$

II. ft. lutio.

To be applied daily after fomenting with cold water. Instcad of poulticing, the wound may be sponged occasion- 
ally with warm water (carbolised if desired) for a day or two, and in the intervals, after carefully drying, carbolised oil ( $\mathrm{I}-40)$ may be applied.

A good application for broken knees which do not seem disposed to heal readily is red iodide of mercury ointment, I in S, applied on lint and held in place by a bandage, redressing every second day. This is good in many other indolent wounds, such as cracked heels, and similar sores.

Wounds in horses, cattle, and sheep (not dogs) should be treated with-

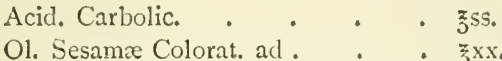

$$
\begin{aligned}
& \text { I. ft. lotio. }
\end{aligned}
$$

This will prevent suppuration and cause union by adbesion in small wounds that have not been exposed to the air, and for old ones it will do good. It takes the sting out of finger-cuts marvellously. For old and gangrenous wounds, or for maggoty sheep, \&c., it may be made twice as strong.

Blisters. - The practice of blistering horses is ancient, but shows no sign of going out of fashion, as fresh agents are from time to time introduced. None, however, are so well esteemed by practical veterinary surgeons as the various combinations with hyd. biniod. rubr.

For a splint or spavin :-

Or-

$$
\begin{aligned}
& \text { Hyd. Biniod. • • . . 弘. } \\
& \text { Adipis . . . . } \xi^{\text {vij }} \\
& \text { M. ft. ungt. }
\end{aligned}
$$

Pulv. Cantharid. . . . . 3 j.

Digest in lard, 8 oz., on a water-bath for 6 to 8 hours; strain, and when it begins to set mix with it

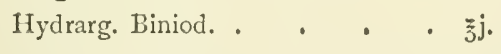

For a thickened tendon or slight sprain, or to brace a joint, reduce wind-galls or soft enlargements, one of hyd. biniod. to twelve of lard or vaseline may be used. 
A simple cantharides blister may be made by digesting cantharides $\mathrm{I}$, resin $\mathrm{I}$, and lard 7 , for 6 to 8 hours, and straining.

\section{Liquid Blisters.}

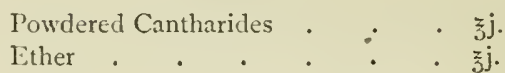

Iour the ether over the cantharides, and let it stand for a day in a

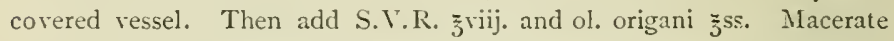
eight days and filter.

A useful preparation in splints, side-bone, ring-bone, spavin, or other bony deposit, is-

Hydrarg. Perchlor.

S.V.R. .

II. et $S$.

When used carelessly this is extremely liable to cause destruction of the hair-bulbs and subsequent baldness - a very serious objection in any but competent hands. A small quantity is to be lightly rubbed into the affected part.

Charges are used much as plasters are in human practice, and considerable benefit is derived from their judicious employment. They should be made liquid in a water-bath and applied as warm as the animal can well bear them. Youatt's formula is a good onc, as follows :-

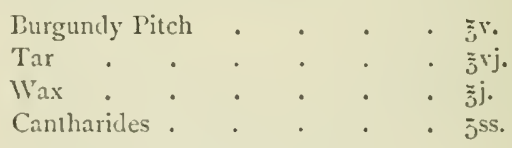

Cleansing Drenches.-In some districts where so-called milk fever is much dreaded, there is a call for cow-drenches both before and after calving, and we would advise the simplest of all aperients to be given beforehand-say a week, or even less -avoiding ergot, which should have a place in the after-calving or 'cleansing drink.' 'There are a few fortunate individuals, chemists and veterinary surgcons, who cnjoy a great reputation for drinks that prevent milk fever; but the secret is not in their private formula, but in getting stockowners to kcep the 
in-calvers low. Six weeks on oat straw and hay, and two or three doses of mag. sulph. (flav., vel rub., vel cerul.), within a little while of calving, will bring a local reputation to anyone who can persuade his clients to adopt the regimen. (See also p. 17 1.)

An old-fashioned recipe for a good drench before calving is as under: -

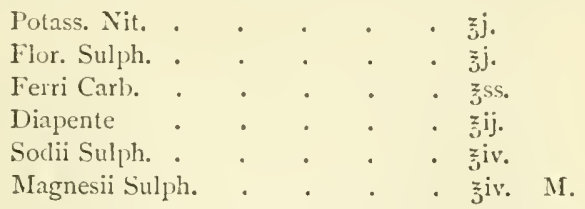

Divide into two doses, and administer each in a quart of gruel.

For a drench to be given after calving the following is a good formula suggested by an eminent F.R.C.V.S. :-

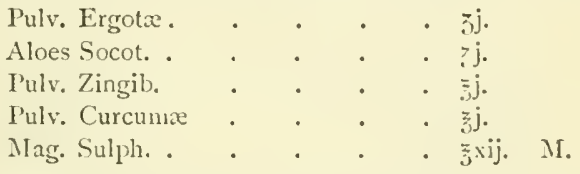

Directions.--The drench. To be given in a quart of hot ale or thin gruel within two hours after calving.

Alternate ten-drop doses of aconite and belladonna after calving is the treatment adopted by some, and it is approved by the Imperial Live-stock Insurance Company (in their printed recommendations to assurers) and is reproduced here for those who have faith in homoeopathic treatment of animals. The reader is, however, referred to Milk Fever (see p. ís).

\section{Tonic Powder for Cattle.}

Sodii Iyposulphit. l'otass. Nit. Pulv. Zingib. . I'ulv. Nifc. Jóm. Pulv. Gentiane

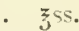

- 引ss.

- $3^{\mathrm{i} i \mathrm{j}}$

- 5 j.

- 气ss. 
One twice a day in treacle and water, gruel, or old ale. Especially useful in debility in cows after calving, or where appetite is deficient, and after exposure of any kind, as in marts and fairs.

Cordial Drench. - For cows not chewing the cud properly or failing in milk, or bullocks off their feed or not thriving, the following is recommended:-

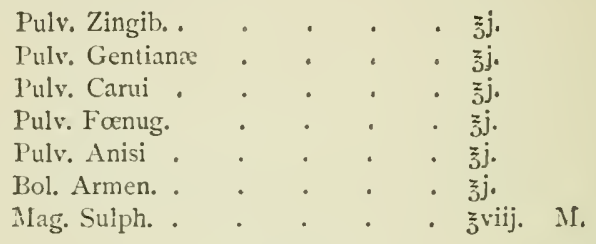

To be given at night in a quart of hot ale or thin gruel.

Calves' Cordial; also the best medicine for diarrhœa in pigs :-

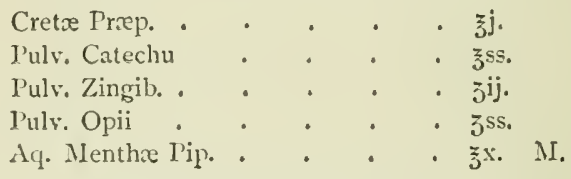

Dose : One to three tablespoonfuls twice a day.

For a pig give $\frac{1}{2}$ oz. to I oz. twice a day; a teaspoonful will be enough for sucking-pigs.

Astringent for Scours or Diarrhea for a Horse or Bullock.

Alum. Sulph. $\quad \cdot \quad \cdot \quad \cdot 3^{\mathrm{ijj}}$
I'ulv. Catechu
Pulv. Cort. Querc.

Fiat pulviy.

To be given in gruel, and repeated if necessary.

\section{Calves' Cordial for Scours.}

Tinct. Opii • . . . 3 ij.

Infus. Catechu • . . . $3^{\text {ij }}$

Alum. Sulph. • • . . 3 j.

Infus. Quercûs arl · • • • 亏ँv.

M. ft. nist. $\frac{1}{6}$ pt. ter dic. 
Or this-

Tinct. Opii . . . . 今̄j.

Aq. Calcís . . . . . Oij.

I. ft. mist. Capt. żij. bis vel ter die.

The old-fashioned chalk and opium mixtures have many friends, but the above have the advantage of not clogging digestion after the astringent effect has been produced.

\section{Dog Medicines.}

\begin{tabular}{|c|c|c|}
\hline \multicolumn{3}{|c|}{ Distemper Pill. } \\
\hline Pulv. Jacobi . & . . . & gr. ijss. \\
\hline Hyd. c. Cretâ & . & gr. ij. \\
\hline Pil. Ipecac. Co. & . & - gr. $\mathrm{iij}_{\text {. }}$ \\
\hline Quininæ Sulph. & . & - gr. jss. \\
\hline Excipient. ad & iI. ft. pil. & - gr. x. \\
\hline
\end{tabular}

One to be given twice or three times a. week.

$$
\text { Another Distemper Pill. }
$$

Hyd. Sulph. Flav. • • • gr. ss.

Gum. Galban. • . . . gr. iij.

Pulv. Jalapæ . . . . gr. ij.

Excipient. ad . . . gr. x.

One twice a week.

$$
\text { II. ft. pil. }
$$

\section{Alteratize Pill for Dogs.}

To be given in conjunction with skin lotions for eczema, \&c. :-

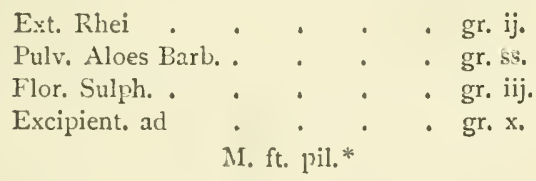

One pill three times a week.

* Hyd. c. cretâ has teen deleted from this formula, which will be fu und in previous editions. Dogs are so easily salivated that it is advisable to do without it. 


\section{Astringent Dog Pill.}

Pulv. Opii . . . . . gr. ss.

Pulv. Alum. . . . . . gr. ij.

Pulv. Catechu . . . . gr. ij.

Pulv. Amyli . . . . . gr. ir.

Excipient. ad . . . . gr. x.

M. ft. pil.

One to be given twice a day.

$$
\text { Purgatice Pill for Dogs. }
$$

Pil. Hydrarg.

Ext. Colchici Acet. • . . gr. $\frac{1}{4}$

Ext. Hyosc. . . . . gr. j.

Ext. Coloc. Co, . . . gr. v.

One for a dose occasionally.

\section{Congh Pill for Dogs.}

Pulv. Camphoræ . . . . gr. $\frac{1}{4}$

I'ulv. Ipecac. Co. . - . . gr. jss.

l'ulv. Scillie . . . . . gr. jss.

Ext. Digitalis . . . . gr. $\frac{1}{8}$

Pulv. Gum. Anmmoniac. ․ . gr. ij.

Pulv. Digitalis . . . . gr. $\frac{1}{8}$

Excipient. q.s. ad. . . . gr. x.

M. ft. pil. Capt. j. bis die.

\section{Ilorm Pill.}

Santonini . . . . . gr. ij.

Vitrei l'ulv. . . . . . gr, iij.

Pulv. Arecx. . . . . gr. v.

Pulv. Jalape • . • • gr. v.

II. ft. pil. I'ro re natà.

\section{Tonic Dogr Pill.}

Ferri Carb. Sacch. . . . gr. ij.

()uinina Sulph. . . . . gr. ss.

Ext. Gentiane . . . . gr. ij.

Excipient. ad . . . gr. $x$.

M. ft, pil. j. omni dic. 
Dog pills are more easily given for being bulky; hence the frequent repetition of 'excipient. ad gr. $x$.'

Hoof Ointments. - As the demand for these is likely to continue, formulæ are here given, but there is a consensus of opinion among the more adranced reterinarians that greasy applications are not merely undesirable but positively harmful. The alternate absorption and evaporation of water has a beneficial effect upon the hoof in the normal state. If the foolish custom is persisted in of rasping the outer layer off the hoof, in order to make it ship-shape, then an unctuous dressing becomes necessary, to replace the qualities for which the outer layer (periople) is remarkable.

The outer layer of horn on a horse's foot becomes hard from being the oldest layer and farthest from the secreting surface. The hardness is for a double purpose-for protection, and to keep pliable and tough the deeper layers. If it is remored the deeper layers become dry, and consequently brittle, thus predisposing the foot to 'sand-cracks,' \&c.

Hoof ointments, for convenience, must have a certain consistency, so as not to spill like oil if accidentally upset, and yet not to be so hard as to require warming for use. They must not be 'sticky,' as in that case the brush with which they are applied gets clogged, and straws and dirt stick to the foot; for this reason, wax should not be added to any hoof ointment. They must be of a dark colour, and for this purpose tar is a useful ingredient, besides its beneficial action upon any 'thrushes' which may exist. Lastly, they must wash off, so as not to interfere with the ready cleaning of the horse when necessary. 'This last quality is not absolutely essential to a good protecting hoof ointment, but its advantages must not be overlooked by those who wish to sell an eligible article. Perhaps, in giving formulæ, it would be better to divide them into two varieties :-

Those used for bad feet as a protecting agent, and which contain no saponifying ingredient; and 
Those which are used regularly as preventives and, like hair dyes and pomatum, are 'beautifying agents.'

The following are good of the first class :-

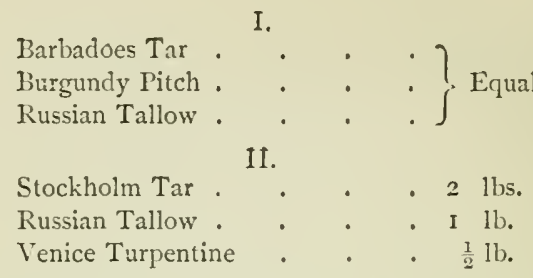

In mixing these, melt the last two ingredients together first, then add the tar and thoroughly mix.

The following preparations are samples of the second class :-

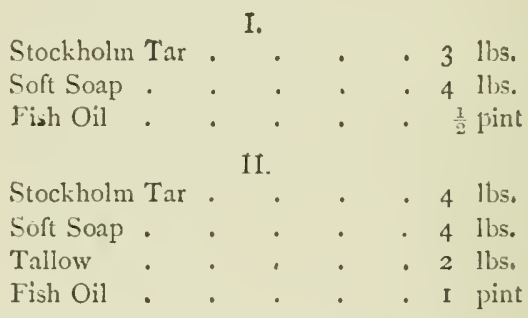

The second is preferable, as being of the better consistence. It is true that alkalies injure horn, but in these forms the excess of fats prevents any marked effects. Glycerin with fats would obviate all objections, but the difficulty is to get a mixture of the proper consistence and colour that would wash off pretty easily. Soft soap by itself is not a good application, as it tends to make the hoof brittle.

Lotion for Cracked Heels, \&c., commonly known in Scotland as the White Bottle. This consists of-

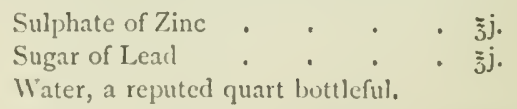

This is also largely used for sore backs, shoulders, and the like.

Treatment of Warts.-Nothing in animal life is more 
remarkable than the sudden appearance and disappearance of warts of various kinds. IVarts on animals vary from the size of a pin's head to many pounds in weight; their appearance is often not noticed till they attain a great size, hence the belief in their mushroom-like growth. 'They may be found on every domestic animal at times, though they are not common to sheep, and pigs.

Warts (zerruce) are technically described as epidermic or epithelial tumours. 'They are made up of layers or scales, but often attain large proportions, and develop within themselves blood-vessels of considerable size. They may be roughly divided into three classes, requiring different treatment :-

Ist. Those growing within a shell or cyst, like a nut, whose shell needs only to be laid open to release them. A sharp knife and a bold cut, followed by a squeeze, will make such encysted tumours or warts jump out clean.

2nd. Pediculated warts. - These may be any shape, but have a narrow neck or base, the attachment being the smallest part of the growth.

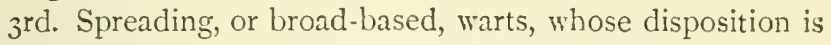
to coalesce and occupy a large surface, in contradistinction to the pediculated.

Scientific pathologists may object to this classification, but it will answer our purpose best.

Warts are found in all sorts of situations-in the mouth of the dog, on the teats of cows, the sheath of horses, the genitals of mares; on the face, eyelids, or other parts of the anatomy. They require somewhat different treatment according to situation.

The first variety require only the knife; the second can generally be got rid of with a tight ligature, but the place should, if situated about the body or limbs, be touched with a red-hot iron or one of the caustic lotions hereafter to be mentioned. Ointments are objectionable, as they afford by their greasiness a certain amount of protection where we want the most caustic effect.

In selecting a ligature the size of the wart should be taken into consideration. A small wart on a pug dog's eyelid, for 
instance, should be secured by fine but strong silk, while a large wart on a bullock or a horse may need a stout waxed end. When ligatures fail, it is usually because they give, or are not at first tied tightly enough. Their effect may be greatly promoted by first wetting and dipping the hair, silk, cord, or waxed end in hyd. sulph. flav. or hyd. bichlor. pulv., or, if a plain piece of string is used, a little pulv. resinæ will help it to hold. There is nothing better for the purpose than broken fiddle-strings which have already been well stretched.

Many of the larger warts can be removed by grasping them in the hands and twisting them off roughly; the vessels are thereby drawn out, and less hæmorrhage follows than would be the case in a careful dissection. They may or may not come again-it is impossible to say with certainty.

The spreading, or broad-based, warts are the most difficult to eradicate, and have a knack of coming again in such abundance that the unfortunate owner is inclined to think wartseed must luve been sown on the last occasion of scarifying. If upon manipulation the warts are found to liave no deep or firm hold, it will be better to secure the animal and dissect them out at once. A large wound need not be feared. The skin will stretch as much as is needful, and can be brought together over almost any wound.

It may be necessary to use a little tinct. ferri perchlor. as a styptic, but we should prefer zinci chlor. $5 \mathrm{j}$. in aq. $\overline{5} \mathrm{iv}$.

Warts of the broad-based kind that cannot either be ligatured or operated upon with the knife may be destroyed in various ways, and among the most successful remedies may be named acid. nitric. fort. and acid. sulph., but they are such dangerous agents in the hands of clumsy carters and cowmen and horny-handed labourers that we prefer such agents as will not destroy the skin if spattered upon another part. Hydrarg. sulph. flav., and cren hyd. bichlor., in powder, are safer than acids, and effectual if the cowman or groom will thoroughly soak the wart in warm water, or soft soap and water, before applying the powder.

The truly marrellous manner in which warts come and go 
has already been referred to, but their cure by the application of warm blood has not been mentioned in former editions of this work, as it was thought to belong to the category of charms, having no practical interest for the pharmacist. 'The theory of infection where the blood of a wart runs over the adjacent skin, too, was tabooed by medical authorities, but it would seem, from correspondence in the Field and other journals, that both these popular notions are justified, if not absolutely proved, by trustworthy experiments. The treatment of the myriads of warts in dogs' mouths has also become practicable by painting the affected parts with a strong solution of salicylic acid. Evidence of a fairly reliable character is also to hand as to the curative effects of salicylic acid administered internally in the case of cattle having warts under the belly and other places where manual interference would be most difficult.

If a lotion is desirable, there can be no more effectual one supplied than-

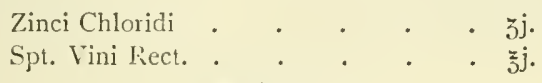

M. ft. lotio.

If this is applied daily after soaking the wart, it will very soon be destroyed, no matter how large.

Dogs are also the subject of pigmentary warts, or small tumours, which require much the same treatment, but rather more knife and stick chloride of zinc. Black-and-tan dogs and Yorkshire terriers, whose coats change colour with years, are the most frequent subjects.

Grey horses, especially dapples, are also subject to pigmentary tumours, which must not be too hastily assumed to be warts or treated as such, particularly if situated about the anus or vagina. These are usually malignant, and if removed tend to recur (melanosis).

Warts on the teats of cows are often rery troublesome, and the milker's hand being applied twice daily makes it difficult to do anything till the cow is dry ; but ligaturing one or two at a time may be tried by anyone with sufficient patience. 
Many a bucketful of good milk has gone down the drains through the pain caused by warts, and they should be removed as soon as opportunity serves.

Foot Rot Applications.-(I) Equal parts of liq. antim. chlor. and tinct. myrrhæ. (2) Equal parts of sulphate of copper, gunpowder, and lard, Finlay Dun. (3) Sol. zinci chlor. (Burnett's Fluid). (4) Acid. sulph. I, ol. terebinth. 8, Hogg. (5) Hydrargyri $5^{\mathrm{ij} .}$, acid. nitric. 3 ij., acid. hydrochlor. $z j$., acid. acetic. $z$ j. To be diluted with from 4 to $\mathbf{I} 2$ parts of water as the case may seem to require, Brown. (6) A mixture of sulphate of copper and Stockholm tar, Fleming.

Foot Rot Pastes.

( I)

Cupri Sulph.

Ferri Sesquioxid. . . . . $\overline{3}^{i v}$

Acidi Acetici Glacial. . . . . ziij.

Glycerini • • • • •

Make a paste with ol. lini.

(2)

Alum. Exsicc.

Hydrarg. Perchlor

Acidi Carbolici . . . . . $3^{\mathrm{ij}}$.

Vaselini . . . . . . $\bar{z} v j$.

Cretæ Præp. . . . . . $3 \mathrm{j}$.

Reduce the solids separately to powder, and mix with the raseline in which the carbolic acid has previously been dissolved.

(3)

\begin{tabular}{|c|c|c|c|c|c|}
\hline Adipis & . & & . & & \\
\hline O1. Lini & . & - & . & & \\
\hline Resine & · & - & - & • & \\
\hline Ol. Terebir & th. . & . & . & - & \\
\hline I'ulv. Cupr & i Acetat. & . & . & . & . \\
\hline
\end{tabular}

Melt the lard, oil, and resin, and rub the verdigris smoothly in the turpentine. Mix. If too stiff, add more linseed oil.

Mange Lotion.-Nange in horses, cattle, and dogs results from the attack of minute acari, which cause much itching 
and heat, accompanied with scurfiness and baldness of the skin. The treatment consists, first in destroying the acari, and second in absolute cleanliness of the parts affected. No better preparation for horses or cattle will be found than the following, which may be applied twice a day for a short time, and afterwards once a day :-

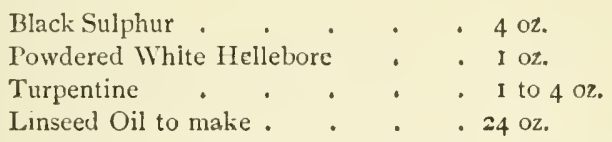

When a milder treatment is required, mercurial ointment or sulphur ointment, or a mixture of sulphur, tar, and linseed oil, may be tried. Another good formula is a mixture of I part each of commercial cresol, soft soap, and turpentine, with I 7 parts rape or linseed oil.

Professor Simonds, formerly principal of the Royal Veterinary College, Camden Torn, was very fond of prescribing the following, and the writer has used it for miany years for dog?, though too vesicating for horses:-

\section{Spirit of Tar \\ Spirit of Turpentine $\quad \int$ Equal parts. Mis.}

Two good dressings, with an interval of three days, will certainly destroy sarcoptic mange. The animal should afterwards be washed with a neutral soap and rinsed in a quart or two of warm water containing $2 \frac{1}{2}$ per cent. of glycerin.

It happens sometimes with dog patients that they must be treated in the houses of their owners, and such an application as the last is not suitable for pets-or, rather, for the upholstery of the house. A fairly effectual lotion for such may be made as follows:-

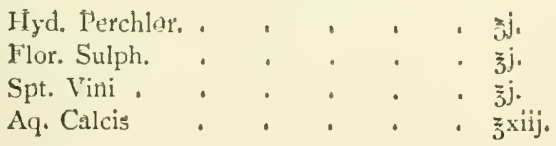

Boil the aq. calcis and flor. sulph. together; dissolve the hyd. perchlor, in the spirit, and add when cold. 
There is another form of mange in dogs, known as follicular, and affecting a comparatively small area of skin. It usually begins on the withers or some spot along the back, very slowly invades the adjacent hair-glands, finding pasture only in those large ones from which grow the coarse hairs which dogs erect when angry. It may spread for a width of two fingers and cxtend from the top of the neck to the root of the tail. It seldom attracts attention until the parasite (Demodex folliculorum) has obtained a firm hold, and is scarcely ever entirely dislodged. The demodex itself can be killed with the tar and turpentine lotion already referred to, but the eggs remain and hatch about the month of July in the following year. The hair should be clipped, and two or three applications of the lotion made, then washed, as for sarcoptic mange; but clients should be made to understand that it will recur unless a bottle of the lotion is kept in stock and applied in the early months of summer, when a disposition to rub against the stares of chairs, \&c., is first noticed.

None of the foregoing preparations are suitab?e for cats, for various reasons, chief among them being the extreme susceptibility of cats to terebinthinous applications. Carbolic acid should under no circumstances be prescribed for cats, and death has not unfrequently been caused by applications of ordinary cresol enulsions. The following is a suitable dressing for mange in these animals :-

\begin{tabular}{|c|c|c|c|c|}
\hline Chinosol & - & & & . I part. \\
\hline (ilycerin & . & & & 2 parts. \\
\hline Aquae I-est. & . & • & . & 200 parts. \\
\hline
\end{tabular}

A previous soaking with soapy water is necessary to remove the serosity in cases of any standing. For the 'common or garden' cat, not sharing my lady's boudoir, ung. sulph. simp. will do well cnough, but a little ol. anchuse and a few drops of eucalyptus will enhance its value in the eyes of most customers. 
Spring Medicines.-It is one of the features of veterinary trade that the advent of spring brings an increased demand for horse and cattle medicines, and it will be advantageous for the retailer to know the nature of the business he has to cope with. It is about this time that the horse changes his coat, and he feels the benefit of alterative powders, for which most country druggists have some cherished recipe. To those who have not we may say that those powclers are most likely to ensure fame which contain remedies that act (1) on the skin in casting the old coat ; (2) on the liver, clogged by months of 'hard' feeding without green meat; and (3) on the parasites which find a home in 50 per cent. of horses in town and country. For a powder that is safe and reliable in these various respects the following is an excellent combination. It should be given in the morning feed, either on damped corn and chaff or in a handful of bran mash :-

Alterative Pozeder.

Antim. Pot. Tart.

Ferri Sulph.

- 35.

Iyd. c. Cretâ

Santonini

Sodii Chlor.

I’ulv. Fonug.

Pulv. Gent.

- $3 \mathrm{j}$.

- gr. $x$.

- gr. $x$.

- $3^{i j}$.

- $3^{i j}$

- $5 \mathrm{ij} . \mathrm{M}$.

This is for a single dose: The mixture may be coloured with Armenian bole without making it unpalatable or interfering with its 'nose'; or yellow with turmeric.

An equally efficacious ball, for those who will pay the better price which balls command, can be made of $5 \mathrm{j}$. of aloes, and with cinchona instead of gentian; but balls containing aloes should only be given two or three times a week, for fear of causing nausea or purging.

It should be remembered that all worm-medicines are uncertain in their action, but the springtime farours the medicaments, as early green meat assists in the dislodgment of the enemy. Such stickers as bots may be prudently pre- 
scribed for in the early summer, as their time has then come, and many an old farrier's remedies enjoy their reputation through being recommended at the right season.

\section{Worm Balls.}

(I)

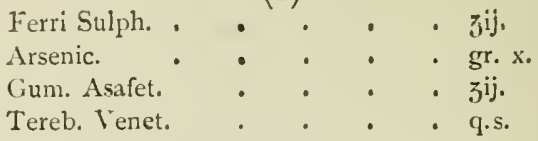

Ft. bol.

(2)

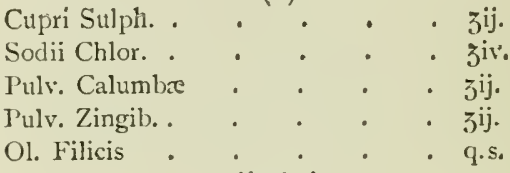

Ft. bol.

One of either of these balls may be given three nights a week. Modern veterinary writers speak of arsenic as dangerous, but it has not been proved so in practice, and many practitioners give a much larger dose than the above with excellent results.

The spring sunshine brings out the lice upon cattle, for which the following is a cheap and efficient remedy :-

\section{Lice Oils.}

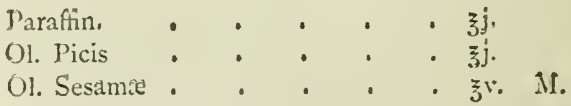

Any damaged or inferior oil can be used for this application.

In coarse-bred horses grease has a tendency to break out in the spring, and for this complaint an effectual remedy yielding large profits may be found in the commercial sulphate of zinc. It should be rubbed down and tinted with a few drops of cochineal, as this will not be enough to stain a white-legged horse. A 4-oz. packet is enough for a gallon of lotion. Balls should be given internally at the same time. Most country druggists have a good grease-ball recipe, and 
we may remind them that any lotion they may prescribe will be much aided by drachm doses internally of either cupri sulph., cupri acetas, zinci sulph., or sulph. nig. Powders can be recommended where customers will not pay the price of balls or cannot administer them. Most horses will eat such unpalatable remedies as cupri and ferri sulph. pulv. if given with salt or fenugreek in a mash, or even on damped chaff or corn. Carters especially have a great preference for powders, though it necessarily limits the prescriber's choice of agents.

Although spring is late for lambing, there is still right up to the end of April a demand for certain things, such as mixtures for straining in ewes, lambing oils, \&c., and these should not be put out of sight. Scouring mixtures for calves are useful all the year round, and will keep, and as soon as the sale of these things is falling off retailers should be making preparations for fly-powders, sheep-dips, and remedies for scabs, maggots, wounds and injuries. Cleansing drenches ought at all times to be in a position to catch the farmer's eye, or his wife's, who is very often both prescriber and dispenser of the remedies used for farm stock.

In districts where quarter-ill or black-leg is to be found the spring is the time to recommend the following:-

\section{Black-leg Preventive Drench.}

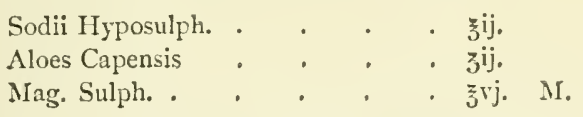

For one drink. To be given in a pint of warm water a day or two before turning out to pasture.

Double this quantity may be given to a full-sized bullock, but the dose indicated is for yearlings, since these are the most frequent subjects of the fatal malady. In the section of Miscellaneous Formulæ at the end of the volume there are numerous prescriptions which fit in with the foregoing remarks.

Ringworm, White Flaw, \&c., should be got rid of without delay, and the dresser should be adyised not to break 
the scabs, lest he catch the infection. The white patches should be liberally anointed with any grease containing sulphur ; here, again, is an opportunity to use up discoloured vaseline, rancid cold cream, or aught else in the way of grease, since it is only a vehicle, and of no therapeutic importance. Such preparations should be coloured and 'nosed,' and, we need hardly say, made the same to the eye from year to year. The following is a useful formula :-

Ringie'orm Oiniment.

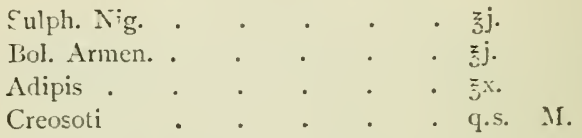

Any essential oil which has gone wrong may be used, but creosote is not only powerful to mask rancidity, but is an active insecticide in itself.

Ung. potasse sulplouratx ( $\mathrm{I}$ in $\mathrm{I} 6$ ) is also a good remedy.

Ringworm patches should not be scraped with the finger. nails, or by other means, as cases have occurred in which the person so doing has contracted ringworm and suffered very screre consequences. The agents above mentioned will prove quite effectual if properly applied.

It 1 ot unfrequently happens that some very good liniment or embrocation is used by coachmen or others for the wrong purpose, so that the skin of a horse's leg is blistered and 'filling' of the limb follows. Simple sprains and injuries are much better treated with an evaporating lotion, which is also a rapid nseans of undoing the mischief of these embrocations, whose active ingredients consist of turpentine, ammonia, \&c. A suitable remedy for all kinds of sprains, injuries, swellings, $\mathbb{S} \mathrm{C}$, of the limbs of horses or cattle is the following :-

\section{Sedatize Lotion.}

Ammon. Ner.

Acicl. Acetic. . . . . . $3 \mathrm{j}$.

I'ot. $\wedge i t$.

Sit. I ini lice.

Aq. al.

II. 
This is a concentrated lotion, and needs to be mixed with a gallon of rain-water, and applied on wet bandages, or sponged on to the injured parts and allowed to dry. There is no season of the year when this may not be sold, if kept in stock and recommended, when less suitable but more widely known goods are asked for.

Another valuable and much-overlooked application is carbolised oil, I in 25 or 30 . This, coloured with either ol. viride or alkanet, will make for itself a demand if sold during the shearing season as wound oil. Any cheap oil may be used as a diluent of the acid.

An Ointment for the Destruction of W'arbles in the backs of bullocks would sell well in the summer if conspicuously labelied. Miss Ormerod has succeeded in calling the world's attention to the annual loss sustained by these pests, though veterinary writers have shouted in vain for a century to the same effect. Ung. hydrarg. rubbed into the tumour is a safe and effectual remedy, and chemists and druggists might exterminate warbles, as well as bots, if they would more persistently call the attention of farmers to the remedies that will prevent their propagation.

An application much needed by the horsekeeper is a cheap and colourless agent for keeping flies from settling on horses. There is a fortune in a really efficient, cheap, and easily applied remedy that may be used over the face without fear of injuring the eyes. Various things have been tried, such as walnut-leaves, infused weak preparations of creosote, Jeyes' fluid, \&c., but they soon dry, and fail to afford assistance. The worst of horse accidents occur from the annoyance that flies cause, and the R.S.P.C.A. would probably confer its gold medal on the man who could produce a really successful remedy.

Setoning is still largely practised in so called 'pooky' districts, and a suitable dressing for inducing and maintaining suppuration in the dewlap (where the seton is inserted) may be composed of ol. tereb. I part, ol. anchusæ 2 parts. 
Sheep Dips.-In the section on Diseases of Sheep we deal fully with the treatment of the various skin diseases which affect sheep. Here it will be well to give some attention to the business side of the matter, for it is a most important one to the country chemist. To enumerate the various insecticides which have from time to time been used as a means of cleansing the fleeces of sheep and freeing them from parasites would be simply to make a list of all poisonous substances generally known. Many of them are now in disuse, and those that are chiefly employed comprise only a small list of poisons, chief of which is arsenic. In some form or other this is the principal ingredient in 'poisonous' dips.

Amongst the so-called 'non-poisonous' ones, carbolic acid or cresol is, again, with perhaps the exception of tobacco, or in some rare cases stavesacre, the main ingredient, under whatever name it is placed upon the market. We propose here to give a few formulæ for the preparation of both varieties.

Of the arsenical variety of dips the following will be found useful and comparatively cheap, and it has the merit of being easily prepared and put up for sale without any elaborate plant :-

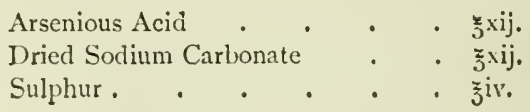

Mix.

This quantity in packets can easily be retailed at from $8 d$. to Is., and is sufficient for twenty-five to thirty sheep, first dissolving in a few gallons of boiling water, and then adding more water to make 30 gallons, to which may be added a few pounds of soft soap if desired. The powder may also be mixed with the soap and sold as a paste dip, when of course the price would be enhanced. Where the price obtainable is not prohibitive, the substitution of potass. carb. for soda is an advantage, but a little more care is then nccessary in the packing to ensure that no deliquescence takes place. 
Another and favourite form of arsenical dip is the substitution of the yellow arsenious sulphide in place of the arsenious acid forming the 'yellow dips' for which so much is claimed; and it is used in the same way and in the same proportions as the first-mentioned variety, while the cost is practically the same.

It is claimed for this class of dip that they not only kill all parasites infesting the shcep, as well as the eggs, but that they are a sure preventive of the 'fly' striking; but this claim cannot be sustained. Flockmasters should be supplied with the fly-powder for which a recipe will be found on p. 209 . Dips of the class above mentioned are improved in respect of repelling the fly by the addition of $\mathrm{I}$ or 2 pints of crude cresol to each 50 gallons of dip. The latter in favourable dry seasons helps to keep off this pest considerably.

Of the many forms of so-called non-poisonous dips carbolic acid in some form or other is the principal ingredient, and is no doubt a good effective dip, and may be either compounded in the form of a fluid or paste, the first form of which is effectively represented by any of the 'soluble cresols' purchasable at, say, about is. $6 d$. per gallon, retailing at from $2 s .6 d$. to $3^{s .} 6 d$. - this quantity being sufficient, with 50 gallons of water, for fifty sheep. It compares farourably as to price with the arsenical form.

The paste dips of this class are, as a rule, simply a cheap carbolic soap, containing from to to 25 per cent. of acid, of which about Io lbs., dissolved in about 40 to 50 gallons of water, is the usual quantity for fifty sheep. It may be noted here that while a certain proportion of soap is useful, too much tends to render the fleece somewhat hard and harsh, thus reducing its market value to some extent, which must be guarded against. Sometimes in place of soap the acid is mixed with a common quality of glycerin, forming what is known as a glycerin dip.* This is undoubtedly a useful

* Glycerin dips have fallen out of favour on account of the attractiveness to fly of the glycerin which remains on the fleece when the sheep have become dry again, 
preparation when the acid is in the proportion of not less than I to 2 of glycerin, of which 12 lbs. would be the quantity for about fifty sheep, in the same quantity of water as for the others. In place of carbolic acid, 'tar cil' is sometimes used, but is not to be recommended, as, although an effective parasiticide, curing mange and 'scab,' it has the disadvantage of not mixing well with water or the other ingredients of the dip, and tends to discolour the wool.

Stavesacre-seed in the proportion of $\mathrm{I}$ to 30 of water, boiling for an hour or more, to which sulphur may be added, is also a useful preparation, although too strong solutions tend to cause nausea and prostration.

Tobacco is another favourite in sone districts, and as the duty-free article is easily obtainable, it makes a cheap and good dip. The following is a reliable formula for ordinary purposes :--

$$
\begin{aligned}
& \text { Unmanufactured Tobacco . . I lb. } \\
& \text { Carbonate of Poiash . . . I lb. } \\
& \text { Sulphur . . . . . I lb. } \\
& \text { Soft Soap . . . . } 1 \mathrm{lb} \text {. }
\end{aligned}
$$

Boil for an hour in a few gallons of water, and then make up to 20 to to gallons, which is sufficient for about forty sheep or fifty lambs. 


\section{DISEASES OF THE HORSE}

Gripes, or Colic.-Perhaps the most common acute disease horses are subject to is colic or 'gripes.' The term is used very vaguely, being applied to nearly every case of abdominal pain, from indigestion up to inflammation of the bowels.

Nearly every stableman recognises abdominal pain, and the majority have strong opinions of their own as to the seat of disease, if they do not know of a nostrum or certain cure. It is almost unnecessary to say that these opinions are not formed on any knowledge of the subject, nor indeed on experience; for when a horse dies he is seldom seen open by the attendant groom or carter, and the cause of death is not always to be easily discerned by a person unfamiliar with the natural condition and appearance of the viscera. It is very misleading to the intending prescriber, unless he be an old practitioner, to be told most positively that the patient is suffering from kidney disease when all the symptoms are obscure, and he would be glad to learn the true history of the case. He cannot ask the patient, and if he listen to the attendant he is more likely to be led astray than if he concentrate his attention on the symptoms, and find out by careful examination what were the special characteristics of a former attack, if the groom is very positive of what is the matter. Information of this kind cannot be obtained from an ignorant man who has been flatly contradicted at the outset, and it is always better to let the attendant do the talking and never be in a hurry to give a decided opinion. A case of gripes may turn out to be anything, from a ruptured liver to a simple bellyache. The indica- 
tions of pain in the viscera are very much alike, and after attempting a general description of the horse's behaviour under such circumstances we may endeavour to differentiate between the causes, and to suggest the most likely treatment to be successful.

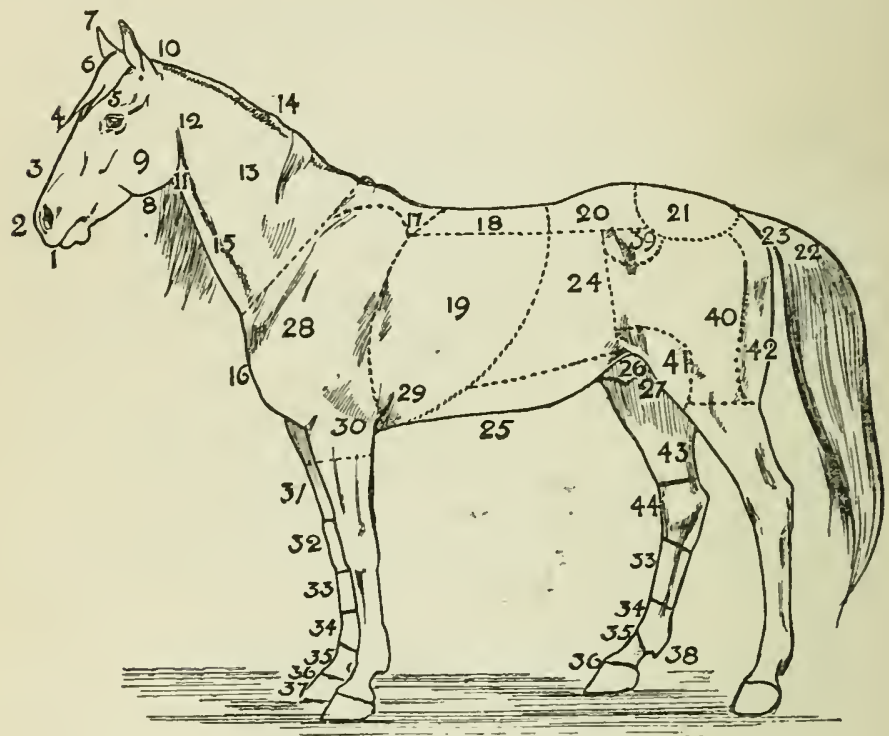

I, I,ips ; 2 , nostrils ; 3 , face ; 4 , brow ; 5 , hollows over the eyes ; 6 , foretop ; 7 , ears ; 8 , lower jaw ; 9 , cheek; 10, poll ; 11, throat ; 12, parotid gland; 13, neck bones : 14: crest ; 15, gullet ; 16 , chest ; 17, withers ; 18 , back ; 19, ribs ; 20 , loins ; 21 , croup ; 22 , tail ; 23 , anus; 24 , flank; 25 , belly; 26 , sheath ; 27 , testicles; 28 , shoulder and arm ; 29, elbow; 30 , fore-arm ; 31 , caston: 32 , knee; 33 , canon; 34 , fetlock ; 35 , pastern ; 36 , coronet ; 37 , foot ; 38 , tuft of fetlock; 39 , haunch ; 40 , thigh : 41 , stifle ; 42 , buttock ; $43, \operatorname{leg} ; 44$, hock.

Horses are more likely to be attacked with colic or gripes after work than during or previous to it, and more frequently in hot than in cold weather.

A copious draught of cold water given to a horse when he comes in from exhausting labour is a frequent source of colic, but provided the water is at something like the temperature of 
the air, there is no objection to a heated animal drinking any reasonable quantity. His blood-vessels are thus rapidly replenished, and the water acts as a restorative, refreshing him generally, and preparing his stomach for digestion. Because drinking spring or well water (generally very cold) is a source of gripes,

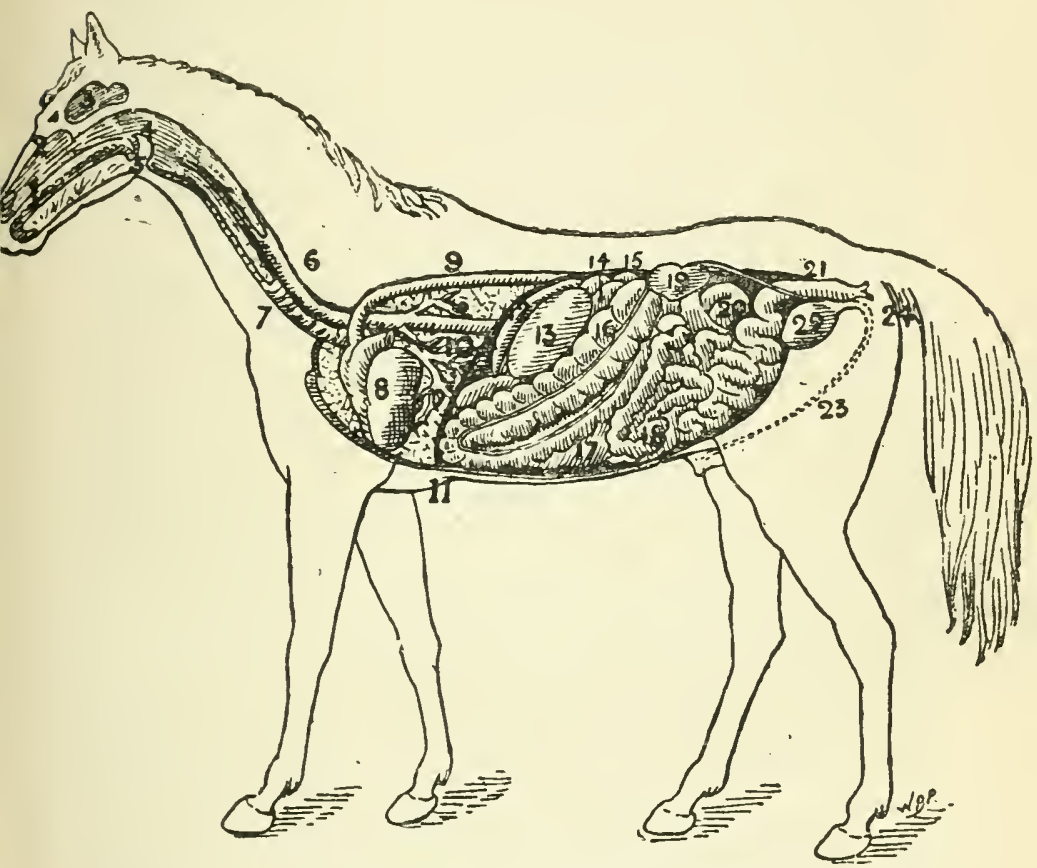

I, mouth : 2, nasal cavity ; 3, cranial cavity : 4, pharynx : 5 , epiglottis ; 6 , cesophagus, or gullet; 7 , windpipe; 8 , heart: 9 , the great abdominal artery: 10, lungs: I1, diaphragm: 12. spleen: 13 , stomach: 14, arterial branches to supply viscera; 15, liver: 16 , the great colon; 17 , cæcum; 18 , small intestines; 19 , left kidney; 20 , floating intestine ; 21 , rectum ; 22 , bladder ; 23 , urethra ; 24 , anus.

it was formerly thought that horses should not be allowed to satisfy their thirst when heated, and until recent years the opinion was quite generally endorsed by veterinary surgeons. It has now been conclusively proved that watering before feeding is best, unless the water is very cold. There has been a 
reduction in the number of colic cases in the Army and the great carrying studs since the latter practicc has been adopted. Gripes or colic may arise from exhaustion, long fasting, and hereditary predisposition, associated with a particular conformation in which there is too much space between the last rib and the hip. 'These are caller] 'washy' horses, and are liable to a variety of digestive troubles, conspicuous among them being colic either of the spasmodic or flatulent varieties.

If a horse is attacked on coming in from work, the first symptom which the groom notices is that he does not want his 'tommy.' Presently he begins to scrape the ground with his front foot, perhaps one, perhaps the other, whisks his tail, stamps with his feet, looks round towards his belly, frequently attempts to make water, appears excited, then dull and listless, scrapes again, brings all four feet close together, and if not racked up will go down after several feints at doing so. Now he will roll on his back, groan and sweat, and as suddenly get up again in an interval of respite, and perhaps begin his meal ; another paroxysm of pain ensues, and the same process is repeated-this is a typical case of gripes or spasmodic colic. If a gripe draught or 'drink,' as it is commonly called, be administered, he probably recovers in an hour or two. The real condition in his case was spasm of the muscular coat of the intestines-extremely painful, as the agonised expression of the horse testifies, but leaving no ill-effects if the diffusible stimulants employed were of a proper kind. Before prescribing, it should be ascertained if anything has already been given, as upon the answer depends the modification or alteration of your dose. If the groom has introduced tobacco into the 'sheath,' or 'figged' the animal, the plug should be remored. In ninety-nine cases out of a hundred the attendant is positive that it is 'his water' that is wrong, and backs up his assertion lyy reference to someone who has seen him pass thick urine.

To those not acquainted with stable nomenclature, we should rxplain that 'figging' is the insertion of a piece of chewed ginger into the rectum; in this case as a remedial agent, though 
more often used by horse-copers to make a sluggish old horse look and behave in a lively and youthful manner at a fair or sale-yard. Of course no good can come from such a remedy, any more than from the insertion of the tobacco in the animal's sheath, and lovers of horses ought to know that 'figging' is a punishable offence, and lose no opportunity of denouncing it. It may also be stated that horses in perfect health, and of both sexes, frequently pass thick urine, and they are much more likely to be wrong when it is colourless than when it is occasionally thick.

In some districts it is common among stablemen and others to give as much as a quarter of a pint of turpentine before secking professional assistance. We are aware, too, that some experienced veterinarians regularly use the following :-

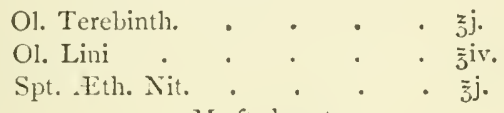

II. ft. haust.

This treatment has the advantage that it pleases an ignorant horse-owner. A horse with violent abdominal pain will not stale; turpentine soon acts upon the kidneys, and thus urged an animal will frequently empty its bladder, though the colic remains uncured; and as, strange to say, numbers of people believe that when a horse has colic 'his water is wrong,' the action of the turpentine is misinterpreted, and the medicine is thought wonderful.

If nothing more than a gill of whisky or other spirit has been given, the following will be a good draught :-

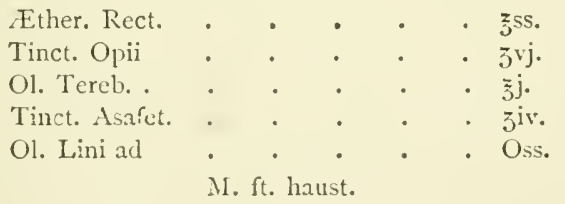

This may be repeated in an hour, if necessary. Considerable benefit is often derived from the following draught, which is 
the one commonly relied upon by many veterinary sufgeons :-

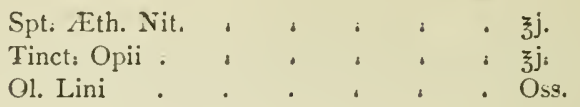

The injection of a pint or two of hot water per rectum is of the utmost service in a case of this kind, but the dung should be first removed from the rectum by introducing the hand as far as the arm can reach. Some little resistance is offered by the sphincter ani, but it is easily overcome by introducing two or three fingers first, and the hand and arm should be well greased, both for the convenience of the operatcr and of the subject. The water used for injection should not be hotter than the groom can bear to put his naked elbow in. This is a fair test for poultices also, the horny hand of the stableman being insensitive to temperatures which might injure either the mucous membrane or the skin of a horse's heel. Not a very pleasant operation; but the man who objects to do it, is not fit for a horse-doctor, however well versed in the art of curative medicine. Injections should not be forced into the bowel, but only gently introduced, for force distends the rectum, and when this force is removed, the gut, like all hollow muscular organs, contracts and expels its contents-an event to be avoided. The longer in reason an injection is retained the better; its immediate and forcible ejection is an occurrence of bad omen.

It is not every case of spasmodic colic which terminates in the happy recovery of the animal in a few hours, and a number of valuable horses die every week of this complaint; it cannot therefore be considered a light matter, and the professional attendant should not be too sanguine of recovery notwithstanding intervals of ease which lead the uninitiated to suppose the difficulty is at an end. The sequelæ to be feared in spasmodic colic are strangulated hernia and intussusception; the former, commonly called twisted gut, or gut-tie, is the result of the muscular spasm before referred to, as also is intussusception, 
in which latter case a portion of the gut is inroluted, like the finger of a glove when pulled off a wet hand.

A fatal result may generally be expected, though it is supposed by some good authorities on the subject that both these conditions are occasionaily remedied by the relaxation of the spasm. As we can only see those cases which prove fatal, it will probably remain a matter of conjecture to the end of time. We know, of course, that human subjects have been successfully operated upon, but horses are not only difficult patients to manage, but much more susceptible to peritonitis, and cannot be persuaded to lie on their backs with surgical appliances for days, and be fed with a spoon. Veterinary surgeons have lately been operating in abdominal cases, but without much success, one of the great drawbacks being the difficulty in arriving at a correct diagnosis in such cases.

It is a tradition of the stable that when a horse is attacked with gripes he should not be allowed to lic down, but con. stantly walked about, and the origin of this is an idea that rolling is the cause of twisted gut; we do not agree with this, but consider a good bed of straw and liberty to seek an easy posture much more conducive to recovery, and if movement of the intestines could be caused by rolling, a gut already twisted might be untwisted in the act ; there is, however, very little doubt that irregular spasmodic contractions of the muscular layers of the intestine are the cause of both the conditions we have been considering.

For other cases, see Flatulent Colic, which has many more causes, and requires different treatment. It is of the utmost importance to distinguish at the outset which of these forms has to be dealt with.

Flatulent Colic.-Under. 'Gripes,' we have treated of cases of spasmodic colic or that form of gripes which is caused by sudden muscular contraction of the bowel.

There are many other causes, besides drinking cold water when the animal is hot, which induce gripes; but flatulent colic is not so often traceable to an immediate and exciting 
cause as to such chronic reasons as indigestion, improper food, over-feeding, long spells of idleness, and fast driving on a full stomach; windsuckers, cribbers, and weavers are the most frequent subjects, though any horse is liable to it. In some it is hereditary as in man, and post-mortem examination does not show any anatomical peculiarity. A knowledge of the fact that it is hereditary should be, but often is not, sufficient to prevent an owner from breeding from a colicky mare. We have known several apparently valuable colts, bred from the same mare, but by different sires, to die of colic without any exciting cause, and before they had ever looked through a collar; the man, therefore, who breeds from a flatulent mare is courting disaster.

Indigestion in one or other of its many forms, and they are legion, is the chief cause of flatulent colic ; but worms, intestinal calculi, dung-balls, and other bodies not carried on by the ordinary peristaltic action, may result in colic; or too much green food, especially if allowed to remain in a heap for some time before it is eaten. It is customary, in the early summer months, to supply horses with cut rye and vetches or other green stuff, which has been cut early in the morning with a heavy dew or rain upon it, and in which consequently fermentation has becn so quickly established that by the time it is delivered in town stables it will be found quite hot upon thrusting the hand into the centre of the bundle. This kind of food is deroured with avidity by stall-fed horses accustomed to dry, hard food for many months at a time, and the consequence is an accumulation in the intestines, fermentation, and climination of large volumes of $\mathrm{CO}_{2}$ and $\mathrm{H}_{2} \mathrm{~S}$. The symptoms very much resemble those of spasmodic colic : the horse paws, scrapes the ground, strikes at his belly with the hind feet, groans, rolls, sweats, and trembles. If he breaks wind to a great cxtent, he scems, and doubtless is, relieved by so much gas as he has becn able to pass; but he is under a difficulty that neither man nor ruminants know; namely; he cannot eructate, save to a very limited extent.

lesides all the differences in a cow's digestive apparatus, 
she is able to get rid of a vast quantity of gas upwards. lou have only to punch one in the belly to discover this, and many cows do it with no greater provocation than being looked at by a stranger.

The pulse in flatulent colic, as in the spasmodic form, is not sufficiently altered at first to be much guide, but it should be noted in order to compare it with some other bowel complaints in which it is.an important indicator. There is one marked symptom which will enable the beginner to distinguish flatulent colic, and that is the drum-like condition of the belly. It is probable that the attendant will have already noticed that, but the reterinary adviser should in any case of colic take notice if the usual depression between the hip and ribs is at all filled up, and whether it sounds drum-like upon being lightly struck. If the case is somewhat advanced and other remedies have already been tried, if the pulse is small and weak and evidence of extreme prostration is present, indicated by cold ears and legs, a bad end may be anticipated, and such a case should not be attempted by any but a qualified veterinary surgeon, if one is obtainable. When there is extreme distension (tympanites) a trocar and cannula are sometimes used to puncture the bowcl; but this operation requires an intimate acquaintance with anatomy, and is usually a dernier ressort of the surgeon. Assuming that the prescriber is called in reasonable time, we would advise a mixture of such remedies as will produce a rapid distribution of nerve-force; that nerve-force which controls the calibre of blood-vessels and directs the operations of the bowels which are not voluntary, and such agents as will form new combinations with the gases, which, we have already said, are chiefly carbonic acid and sulphuretted hydrogen. Ammonia suggests itself at once as most likely to have this effect, and given in large doses, much diluted and often repeated, is frequently marvellous in its reduction of the tympany. As the disease often arises from retained and partially digested food, experience has long since induced farriers to give an aperient with, at any rate, the first dose, and although this practice is questionable on strictly scientific 
principles, we are in this instance disposed to think the old rule-of-thumbers are right.

The following for a first dose has been most successful in our hands :-.

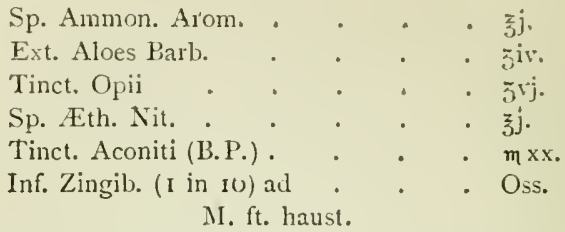

Fleming's tinct. aconite is much used in veterinary prac. tice, but in this work the P.P. preparations are adhered to as much as possible, for the sake of convenience and uniformity. The following is also worth trial :-

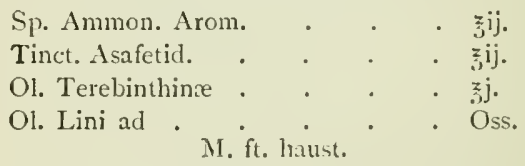

A stimulating application all over the belly is helpful by exciting the peripheries of the nerves, and no doubt this is the cause of the benefit derived from the application of topical remedies for affections seated in parts which would seem too remote to be benefited. Elliman's Embrocation or white oils serve for this purpose, but the following is specially adapted in the cases now under consideration :-

Lin. Camph. Co.

Parts

Ol. Origani . . . . . I

Lin. Saponis . . . . 6

Tinct. Opii . . . . I MI.

If the horse is not improving in an hour from the first dose, the following may be given every hour :-

Sp. Ammon. Arom. . . . . żj.

Ather. Rect. . . . . . jiv.

Tinct. Opii . . . . $3^{\mathrm{iv} .}$

Aq. ad . . . . . $3 \times 1 \%$

M. ft. haust. 
Warm-water injections per rectum should be given frequently, and a blanket saturated in hot water and suspended round the belly should be advised, the bed made comfortable, and an attendant told off to watch the patient and prevent him from knocking himself about.

If relief is not obtained in a few hours, inflammation of the bowels is to be feared.

Constipation.-All domesticated animals are liable to constipation, and wild ones are not wholly exempt from it, though the instinct of the wild beast leads it to eat laxative food, while animals which have been domesticated for generations lose this among other instincts of self-preservation; besides which horses and cattle, if not dogs and cats, have no opportunity of selecting the herbs necessary for medicinal purposes. The stabled horse and the stalled ox eat what is given them so long as they continue to be well, and it is frequently only by their refusal of food that the attendant becornes aware of anything amiss. A good stableman will not think it beneath his dignity to note day by day the character of the freces he removes from the stall, and many a fastidious horse-owner has lost a valuable animal through failing to observe these things for himself.

Constipation in the horse may be said to be always dangerous; unlike the ox and the dog he cannot retain a lot of ingesta for any length of time without grave symptoms. The first and earliest symptoms of constipation in a horse are straining and groaning in the act of defæcation, an abnormal effort and arching of the back, \&c., and the dung when examined will be found to consist of very small balls, more or less glazed and hard; this is the time for a copious bran mash or a small dose of linseed oil. The following is a suitable draught, and calculated to save the life of a horse at this stage :-

O1. Terebinthine
Ol. Anchuse
Ol. Lini .

MI. ft. haust. 
Turpentine is a valuable stimulant and antispasmodic when judiciously administered; it should always be masked in oil. The function of the ol. anchusæ needs no explanation. A drachm of ol. anisi, vel carui, vel menthæ also assists the ol. anchusæ in some stables.

Constipation neglected becomes

Impaction of the Bowels, which in its symptoms resembles or is the cause of gripes. The retained ingesta undergo fermentation and evolve gases which produce flatulent colic, or by their solidity absolutely block the canal and permit the accumulation of gases which should be voided per rectum. (See Gripes and Colic, pp. 59-65.) Impaction is the result of habitual dry feeding and want of bran, carrots, or other laxatives, or of inactivity of the liver or obstruction in its duct. (See Inflammation of the Liver, p. 72 ; and Jaundice, p. 74.) The necessity of a spring and autumn dose of physic, or aloetic ball, is elsewhere insisted on in these pages.

Diarrhœa.-This is not the term used in the stable; 'purging' being the more common word used to express the condition, unless some more forcible but less elegant expression be employed. It is always of serious import : yes, critical reader, always, although you may own a horse whose tendency to looseness is habitual and no harm has come of it ; yet be warned in time and adopt any astringent diet and other means to counteract it of which we shall hereafter speak particularly. 'There are horses known as 'washy,' perhaps in other respects desirable animals; but the moment their harness is put on, they begin to unload the rectum, and as they journey on getting looser and looser, positively purging at the end of a moderate journey, refusing their food, and as a consequence being unfit for work next day, and having that 'tucked up' appearance which every good horseman aroids when making a purchase. Some horses can never be cured of this disposition to purge, as it appears to be connected with a nervous excitability. Such animals are not fit for hunting or hard work in harness; they are show animals, and may be brought out twice a week 
for an hour or two, and require all the remaining interval to regain their appetites and drop their tucked-up bellies. This is serious to a man who buys a horse for work and not for show. These creatures generally come to an untimely end in the hands of some ignorant or careless persons, who bring them in hot and purging, and neglect to look after them when they are by-and-by found to be very ill, suffering from mucoenteritis. (See Enteritis, p. $; 6$.)

For treatment of 'washy' horses a good deal may be done in the matter of diet and drugs in careful hands; but the morality of horse-dealing usually points to an early salc.

Withhold all green food and roots as well as bran ; give no hay that is not old and of the best ; choose sound, heavy oats at a price that will place them above suspicion of being kilndried or musty, or in any way defective, and let a moderate amount of good beans form a part of the daily diet. Beans are the most astringent of all horse foods, and contain the largest amount of nutriment in the smallest bulk; they should be kebbled or crushed, especially for old horses whose teeth may be defective. On a journey, water should be mixed with a little oatmeal and the chill taken off ; careful management will do a lot towards making such an animal useful, but there is no specific for a downright 'washy' horse. A cordial ball as follows, after a journey, will help :-

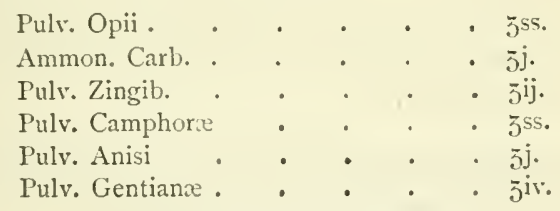

M. ft. bolus. Pro re nata.

Or a draught as follows:-

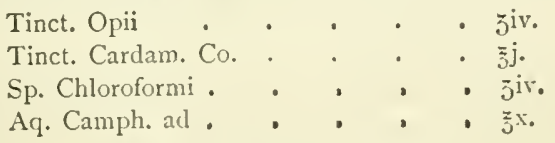

I. ft. haust. I'ro re nata. 
There is no such difficulty in treating diarrhcea that is occasional and accidental. It may be that nature is making an effort to rid the horse of indigestible or improper food, and the store of forage should receive immediate investigation. Whatever the cause, it must not be suffered to continue very long, as horses die of super-purgation, in this, as in many other respects, being more delicate than other animals.

Treatment.-Clothe the body and bandage the legs; withhold all laxative food, such as grass, bran, roots, linseed, \&c., and give the following draught :-

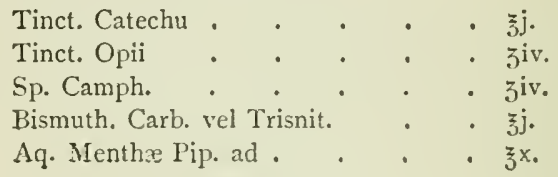

II. ft. haust.

This may be repeated, with perfect safety, every four or six hours if necessary. If a ball is more easy to administer, the following may be substituted :-

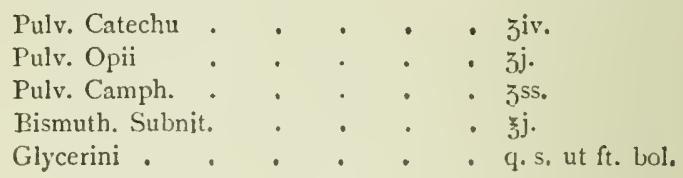

This makes a heavy but not too bulky ball, and most horses can take a very much larger ball than the ounce size that custom has prescribed.

Inflammation of the Liver is said to be infrequent, and it is so, in farm-horses and others, not habitually fed on stimulating diet; but it is not a rare complaint in large towns and among an equine population habitually fed on corn and dry food, stabled in close railway-arches or underground, as often happens where space is valuable.

The symptoms are loss of appetite, scraping with the front feet, occasional lameness of the off fore-limb, hide-bound, semiinsensibility, often stạnding with the head in o comer and 
apparently deaf or wholly indifferent to surrounding objects and noises. The pulse is quick, but feeble; the white of the eye reddish-yellow; the other visible membranes more or less yellow, and tongue sticky. The bowels do not act, and, if the rectum be unloaded mechanically, the dung-balls will be small and glazed, dry, and, when trodden upon, will squeeze out instead of readily falling to pieces.

Tenderness sometimes exists on the right side, and the horse groans when turned round; but these last are not reliable symptoms, as the same occur in pleurisy, a wholly different affection.

The old-fashioned treatment was to bleed and give calomel and aloes, and we should not like to say it was bad; but, now that the lancet has gone out of fashion among the leading veterinarians, it will hardly do for the rank and file to practise it, lest one of the great ones should be called in and condemn the treatment and bring discredit upon the less eminent but not less useful prescriber.

The treatment that commends itself to the present generation is to give enemata of soap and water at frequent intervals, counter-irritation on the right side over the region of the liver, and salines. Mustard may be used as the counter-irritant, though the counter prescriber would do better to supply 'lin. ammon. 5 vj. statim applicand.' A bold aperient dose is also advisable at the outset, since constipation is an obstinate concomitant of liver inefficiency, and retained ingesta frequently cause the death of horses when very little else is the matter with them.

Give a dose of aloes of from jiv. to jviij. according to the size of the animal, with calomel $\bar{j} \mathrm{j}$. as a first dose. The prescriber must not be disappointed if the animal will not eat and shows no immediate improvement, as the aloes will not probably act as an aperient to any extent; but it will just prevent that complete stagnation of the bowels which is the cause of death more frequently than the actual disease of the liver. One of the chief functions of bile is to give that stimulating anction to the intestines which propeis the contents and at the 
same time arrests fermentation; hence constipation follows when the proper quantity or quality of bile is not poured into the intestine.

A saline draught night and morning, containing a stimulating tonic, is advisable, as all appetite is lost, and the circulation is much enfeebled, as may be known by the coldness of the extremities ; sometimes two legs feeling cold, and at others three or four, although the bodily temperature may be as high as $105^{\circ}$ Fahr. taken at the rectum.

\section{Saline Draught.}

\begin{tabular}{|c|c|c|c|}
\hline Mag. Sulph. & - & • & • \\
\hline Potass. Nit. & & - & • \\
\hline Potass. Bicarb. & . & . & . \\
\hline Ether. Rect. & & . & • \\
\hline Aq. ad . & & • & . \\
\hline
\end{tabular}

M. ft. haust. Nocte maneque sumend.

After three or four doses of the above a couple of drachms of ext. gent. should be added to each dose, as there is nothing in the Pharmacopœia that will induce appetite in a horse if gentian fails.

Jaundice is a condition in which the bile enters into the general circulation from some obstruction in the liver or its duct. It is easily recognised by the staining of the visible membranes, as the eye, mouth, nostrils (Schneiderian membrane), \&c. The treatment should be aimed at getting the liver to work, subduing nausea, unloading the bowels, promoting circulation and skin-action by clothing, bandaging, and gentle exercise, green food when obtainable, or carrots in winter; and for medicine-

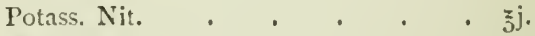

$$
\begin{aligned}
& \text { Mag. Sulph. • . . . . Jiv. } \\
& \text { Liq. Ammon, Acet, ad . . . Oj. }
\end{aligned}
$$

$\Lambda$ bout three such doses if the bowels respond, when rege. table tonics should be resorted to. Much debility follows on 
jaundice, and a subsequent tonic treatment is generally necessary.

\section{Vegetable Tonic.}

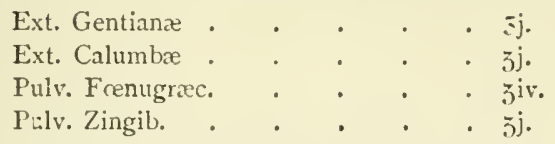

Excipient. q. s. ut ft. bolus. Nocte maneque.

In all affections of the horse's liver there is one common symptom of nausea, denoted by curling of the lip, "turning up the nose,' as is the expression, while the urine is in every case high-coloured.

The practice of drenching horses with gruel, \&c., should only be resorted to when for several days at a time they cannot be induced to eat at all.

There are practitioners who do not hold this view, but we give it as the result of much careful observation. You may indulge a fussy client by letting him do it, but it is doubtful if his interference does not annoy and nauseate the patient when he might otherwise pick over a little food. Sick horses, like sick men, should be tempted with a variety of food.

Stomatitis. - This is a term over which some of our pharmaceutical brethren stumble. It is inflammation of the mucous membranes of the mouth, not of the stomach, which will be found under the head of gastritis. There are several forms of it, into which it is not necessary for us to enter. The chief symptom is the eruption of vesicles in and around the mouth. It has some resemblance to thrush in infants, aged persons, and those in extremis ; varying in its intensity from a simple eruption to ulcerative sores. Bad sanitary conditions and improper food are thought to be the chief causes, and its greater frequency among the young is also noted. Treatment consists in good hygienic conditions, exercise, fresh air, mild aperients, preferably of the oleaginous or saline type, and for mouth washes, or application to the lips, a saturated solution of boric acid (one oz. to a wine bottleful of water). 
Inflammation of the Stomach, or Gastritis, is sometimes met with in horses. When they are suffering from it the mouth is full of frothy saliva, and dribbling in ropes is almost constant. The habitual use by carters and others of irritant drugs may be the cause, but it is a very serious condition when it exists, and the most skilful treatment too often fails to save the animal's life. Gastric sedatives are indicated, and a soft diet, easy of digestion-crushed and scalded oats, carrots, grass, \&c. ; and for medicine-

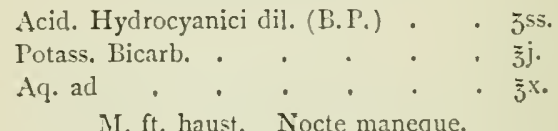

M. ft. haust. Nocte maneque.

The chemical incompatibility of the ingredients in the above mixture need not be considered; the sedative effect is well proved. Diet and rest are of importance; chilled water should be allowed at the animal's discretion.

Enteritis is an inflammation of the mucous membrane lining the intestines. This inflammation may extend and involve the muscular and even the peritoneal coats. It thus differs from spasmodic colic, which affects the muscular coat. It is very fatal, and too oftenlnot recognised in time to be treated with a fair hope of success. Sometimes a shirering fit and the usual accompaniments of a febrile condition arouse the attendant's notice, but it more commonly happens that the animal is discovered scraping with his fore feet, looking round at his flanks from time to time, getting down and up again, and in many respects behaving as if suffering from colic, but not to the ordinary observer so bad. The pulse, which in colic or gripes is not much affected, is in enteritis quick and wiry, the extremities cold, and tenderness of the belly is erinced on pressure, which is not the case in gripes. The breathing is quick and the countenance cxtremely anxious. In colic there are remissions of pain, and the horse will commence to feed until a fresh paroxysm occurs ; but in enteritis 
he gradually gets worse, and persistently refuses food, the pain heing continuous, with paroxysms of excessive severity. Anong the causes of this clisease may he mentioned drinking cold well-water when heated by exercise, east winds, chills, orerfeeding, had hay, musty oats. The absorption of some ptomaine has also been suggested.

Treatment.-Eleeding from the jugular rein to the extent of two or three quarts is often attended with good results; mus. tard applied all over the belly; opium (pulv.) and calomel in doses of $5 \mathrm{ij}$. and $5 \mathrm{j}$. should be given immediately, and after two such doses $\frac{1}{2}$ pint of linseed oil with $\tilde{5} \mathrm{ij}$. tinctura opii bis die. Repeated small doses ( $5 \mathbf{j}$ to $5 \mathrm{ij})$. Tinct. chloroformi et morphina have proved successful in the hands of several practitioners whom we have consulted. Injections of warm water per rectum should be frequently employed. The legs should be hand-rubbed and bandaged with woollen bandages, the ears pulled and a hood supplied; a loose box with the fresh air but no draught, plentiful bedding, chilled water to drink.

Cold, or Catarrh.- Just as a man neglects a cold in his own person, so is the horse-owner apt to disregard a cold in his horse. We are all aware that a neglected cold often ends fatally with other people, but in this matter we act on the belief that 'all men are mortal but ourselves,' and so we assume that our horse will get all right again. But a cold or catarrh is alway's a matter of serious concern in a horse. His great volume of lung-power is not a safeguard but a danger to him, and a common cold is far more likely to do irreparable injury to him than it is to a weakly human being. It is a common observation that robust men, mature or middle-aged, are more likely to die when attacked with pneumonia than are children with much less respiratory capacity. So with the horse : his great lungs are liable to congestion from comparatively small causes, and the results are very serious.

The symptoms of a cold are rigors or shivering, standing with the legs under the belly, all the feet being brought nearer and the back arched; refusal of food, sore-throat, evinced by 
nodding the head and cough, 'quidding' or dropping the food out of the mouth, coat 'staring' or sticking up instead of looking smooth and shiny - these are the preliminary symptoms of a bad cold; when less serere the rigors may not be noticed by any but a good horseman, and the first indication to be observed may be a cough or a cold and running at the nose, with more or less languor and indifference to food. Here is the danger with an inexperienced horseman-he puts the horse to work as usual and brings him home seriously ill. If a cold is observed to be coming on, the horse should not be put to work, but given a bran mash with pot. nit. 3 ss., and mag. sulph. 亏iv. to 5 viij. - the legs warmly bandaged and the stable kept moderately warm, not hot, and by no means draughty, and this will probably be all the treatment required. If successive rigors follow and common fever seems to accompany a cold, a fever draught should be given, the temperature taken at the rectum, and the observation repeated in a few hours, when, if it has run down again, no great good will be derived from internal medicines.

If sore-throat is evinced as well as the above symptoms, a good warm liniment should be applied freely. The following has proved servicealole and can be recommended :-

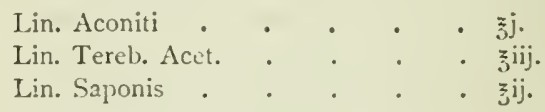

Ft. linimentum.

Mustard applied rather thin answers well for closely-clipped or fine-skinned animals, but for cart-horses and others with a heary coat the liniment suggested can be better depended on to penetrate through the thick and greasy covering. If this produces a sharp reaction, and swelling and soreness to the touch, the cough will probably be rapidly relieved and softened; the swelling outside appears to 'clear the pipes,' and the animal breathes better as soon as the distress caused by the stinging of the liniment has subsided. If the throat 
symptoms continue, a belladonna electuary should be used as follows :-

$$
\begin{aligned}
& \text { Ext. Belladonne } \cdot \quad \cdot \quad \cdot \quad \cdot \quad 3 j . \\
& \text { Potassii Chlorat. } \\
& \text { Oxymel. Scille q. s. ut ft. electuarium. } \\
& \text { Bis terve die si opus sit. }
\end{aligned}
$$

This should be prepared secuntum artem and placed upon the end of a broad spatula as a preliminary to putting it high up on the back of the tongue. It is not a difficult operation if the longue is quietly drawn out of the mouth with the left hand and the spatula introduced with the right. This has given immediate and permanent relief in many very bad cases where to attempt to give a drench or a ball would have brought on a paroxysm of coughing.

We have also seen great benefit in acute sore-throat from homceopathic doses of aconite and belladonna alternately. It is about the net result of some years' observation of homcepathy as applied to animals.

A discharge from the nostrils, at first watery and gradually thickening, is often the only symptom of catarrh apparent to the attendant; this should he sponged off the muzzle frequently and the adjacent parts anointed with a little lard or vaselinc. When we say vaseline we do not mean the crude rubbish that has been sold under that name to veterinary practitioners, and in some cases has actually caused blisters. If the muzzle is anointed frequently, much scalding and discomfort are aroided.

It is also a good plan to give carrots, placing them on the floor rather than in the manger, as the head is better cleared of the discharge ly being kept in a pendulous positiongreen meat taken at such times will often tinge the mucus green, which must not la mistaken for glanders. As sequele from a had cold may be mentioned whistling, thick wind, and roaring, which are discussed in their proper place.

Coughs. - A cough is the symptom of some derangement of a portion of the respiratory organs. That all coughs should 
not be treated in the same manner is evident from the variety of causes. Irritation of the larynx, air tubes, or lung, pneumonia, pleurisy, and some nervous derangements, all give rise to coughing. A correct diagnosis in each case requires a knowledge of some other symptoms besides the cough ; this of itself, however, is so different in sound and quality, depending upon its cause, as to give a fair indication of its nature.

Irritation of the larynx, the most common cause of cough, may be due to inflammation of its lining membrane, or to the presence of a foreign body. In the latter case removal is necessary. But coughing is not always an effort to expel some obstruction; it may be a nervous action depending upon irritation of sentient respiratory nerves. Irritation of the larynx may be acute or chronic. In the acute stage the cough is at first loud and hard, owing to checked secretion; it soon, however, becomes softer, as the membrane is corered with purulent mucus. Diagnosis is helped by the fact that swallowing is difficult, even water, during attempts at drinking, being returned through the nose.

Treatment.-Dry hard food must be avoided ; and in giving medicines do not use the form of balls or draughts. The former are nearly certain to be coughed back, and may get lodged in the nose, an awkward accident, whilst draughts require the head elevating in a manner favourable to choking. Electuaries are the best form. The application of a roll of flannel soaked in hot water round the throat covered with some waterproof substance is often of great service.

As regards medicine, keep the bowels open, and use a sedatire and astringent electuary, as-

Camphor.

Ext. Belladonne.

Acid. Acet. Dil.

Mellis vel Theriace

$$
\begin{aligned}
& \text { jiv. } \\
& \text { jiv. } \\
& \text { - วँx. M1. }
\end{aligned}
$$$$
\text { - } \overline{3}^{\mathrm{ij}} \text {. }
$$

A tablespoonful twice a day, to be smeared on the tongue. Ot this:- 
Tannin

Rectified Spirit . . . . . $\xi^{i j}$.

Honey

To be given as above.

Chronic irritation of the larynx requires different treatment; the cough here is loud and hard, but not so frequent, occurring chiefly when the animal is changed from the stable to the fresh air, or vice versâ. There are no general synuptoms. Cough balls are here, if anywhere, of use. Good formulæ are :-

$$
\begin{aligned}
& \text { Pulv. Opii . . . . . . } 3^{\text {ss. }} \\
& \text { Pulv. Scillre . . . . . 3j. } \\
& \text { Pulv. Aloes . . . . . } 3 \mathbf{j} \text {. }
\end{aligned}
$$

Make into a ball with common mass or linseed meal and treacle. One every day.

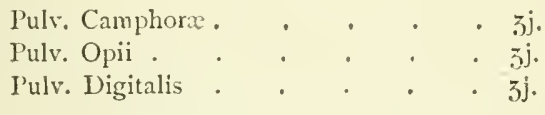

Make as above. One every day.

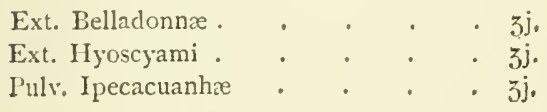

Make and give as above.

Blisters to the throat afford relief in some cases, or setons may be used.

Bronchitis, or inflammation of the lining membrane of the air tubes, is accompanied by a harsh, wheezing cough, accompanied by a loud, rough noise in the windpipe. It is dealt with more fully in the next chapter.

The treatment is general and local. General treatment on the plan recommended for simple fever. Local treatment consists in protecting the neck and chest with rugs, \&r., and in chronic cases applying blisters. In this disease there is an obstruction in the air tubes of purulent mucus, for which as 
expectorants may be added ipecac. and tartar emetic, of each half a drachm, to the following ball :-

Pulv. Camphor.

I'ulv. Digitalis

Pulv. Potass. Nit.

Pulv. Lini sine oleo

Theriacæ q. s. Ft. Bolus
$3 \mathrm{j}$.

$3 j$ ss.

zjss.

$3^{i j}$.

One every day.

There is a cough in cases of broken wind which is almost diagnostic of the disease. It is long, soft, and wheezy. The only other symptom requiring mention is the double action of the flanks in expiration. There is some doubt as to the pathology of this disease. Generally on post-mortem examination of an old-standing case the lung is found to be emphysematous, i.e. it presents littlc bladders on its surface due to a rupture of the smaller air cells ; but this condition of lung is also found in some animals which, during life, showed no symptoms of broken wind. Nor is it invariable in cases of broken wind showing no change of lung. Broken wind is generally due to bad feeding, and musty hay has produced it in a few days.

'The treatment must include attention to diet, which should be of the best quality, and not in too great quantities. Avoid loulky innutritious food; but above all mouldy or dusty hay. Linseed, boiled, and then given cold with the corn every night, has a marked effect in sonie cases. There are two medicines which are followed by good results-arsenic and creasote. Great relief will often follow their use, especially arsenic, which is given in three-grain doses daily, three times a week for a fortnight; then, missing a week, resume for another fortnight. Creasote is given in half-drachm doses in the form of ball, made with linseed and treacle.

Horse-copers are very adroit at what is called 'loading' broken-winded horses. liy some means they produce a temporary relief, during which an animal is sold as sound. This loading consists in giving a mixture of shot and tallow to the animal about half an hour before showing lim. Others trust to a pint of olive oil, while some add a preliminary course of tar balls. 
respiratory apparatus is difficult and dangerous, and we strongly advise electuaries, both for safety, convenience, and directness.

Linseed and bran mashes, scalded corn, damped hay, hay tea, carrots, \&c., should be advised, and everything dry, dusty, or inferior in quality put out of the animal's reach.

If for any reason electuaries cannot be given, the following draught every six or eight hours may be prescribed :-

Vin. Antim. Tart.

Vin. Ipecac.

Tinct. Camph. Co.

Tr. Scillæ .

Liq. Ammon. Acetatis ad 3ij.

- $3 \mathrm{ij}$.

- $3 \mathrm{j}$.

- $3 \mathrm{j}$.

- Qss.

II. ft, haust,

Or this :-

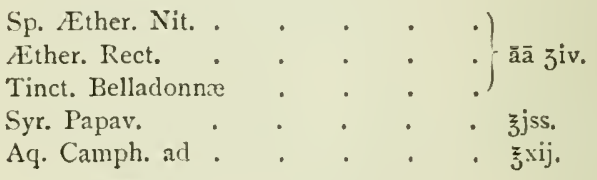

MI. ft. haust. Bis terve die.

Pneumonia is inflammation of the lung substance or parenchyma, and is distinguished from congestion of the lungs, which is engorgement of the pulmonary vessels, and brought about in a different way (see Congestion, p. 95). We are aware that the terms are somewhat mixed in human practice, but they must be carefully distinguished in equine medicine.

Pneumonia is often brought on by a neglected cold, 'invasion,' as it is called ; that is to say, the catarrlial affection spreads instead of abating, invading the ling substance. This will be known by the rapid increase of temperature, short, hurried respirations, redness of the membranes of the eye and nostrils, cold extremitics, dilated nostrils, hanging head, continual standing, and loss of appetite. If the prescriber is called in early, he should apply mustard immediately to the sides of the chest, from the edge of the blade bone to the last rib on one or both sides as may be necessary - a pound is not too 
much for a full-sized horse. The water need not be hot, certainly not boiling, and the mustard should not be mixed too stiff, but well rubbed in, which latter is more easily said than done, since good mustard is blinding to the person who takes long rubbing it in. The day after applying mustard the parts should be rubbed gently with oil to complete the action of the mustard, and soften the corrugated skin. We prefer the camphorated oil of the B.P.

The following draught should be given night and morning, until the temperature falls :-

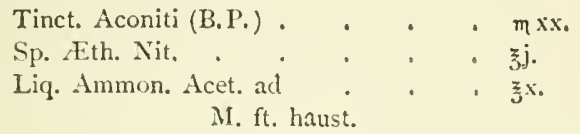

If giving a draught upsets the animal, or if it causes cough. ing, the following ball will be found of service :-

$$
\begin{aligned}
& \text { Pot. Nit. . . . . . } 3 i j \text {. } \\
& \text { Ammon. Carb. . . . . . } 3 \mathrm{j} \text {. } \\
& \text { Gentianxe . . . . . } 3 \text { ij. } \\
& \text { Cinchonx . . . . . } 3 \mathrm{jj} \text {. } \\
& \text { Glycerini . . . . . q. s. ut ft. bolus. }
\end{aligned}
$$

After the first two or three days, when the temperature will be down if the case is likely to terminate favourably, the following should be given :-

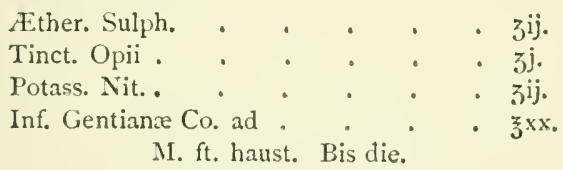

Great prostration, if nothing worse, follows upon pneumonia, and, despite the most careful treatment, a large proportion of patients die either from suffocation by the blocking up of the lungs, or from fluid exudation into the carity of the chest, or from relapse and exhaustion. Puncturing the thorax and drawing off the serum is occasionally performed, but it is doubtful if it ever saved a case. 
In the convalescent stages tonics should be given, but no iron, copper, or other minerals. The following ball is suitable :-

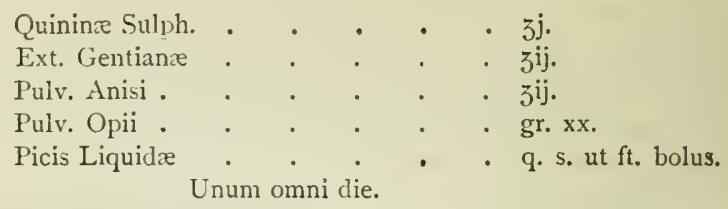

During the early stages of the disease it may be necessary to keep the bowels open with clysters of warm water and soap, but no aperient should be given stronger than mag. sulph. 4 or $6 \mathrm{oz}$., or ol. lini 10 to $20 \mathrm{oz}$., preference being given to the latter unless the temperature be very high. After aconite and pot. nit. we know of nothing that so quickly reduces the temperature without harmful consequences as mag. sulph. Horses recorering from this disease need much care, and should have only gentle exercise for some time.

Pleurisy.--In point of frequency and severity, pleurisy is the most important disease of the respiratory organs of the horse. Though severe no case ought to be fatal if properly and promptly treated. Pleurisy may result from a wound of the chest, inut usually occurs as a sequel to exposure. It may occur on one side only, or on both. It is generally caused by horses standing in a cutting wind when heated, as, for instance, when, after ploughing, the men sit down to their mid-day meal, and leare the horses on a hill-top. Commercial travellers, doctors, and others who drive fast and leave their horses standing about in a draughty street, are apt to pay a penalty in this way. Warm, badly ventilated stables, excessive use of warm food and heavy clothing, predispose a horse to an attack.

The symptoms are much the same as in pneumonia, but the sharp dry cough is different at first, and the animal grunts or groans when made to turn, and flinches when the ribs are pressed; he often looks round at his sides, and this gives rise to mistakes among the inexperienced because it is a symptom 
of gripes. It may be taken as a general rule that horses suffering from inflammatory diseases of the chest do not lie down, while they seek by every recumbent attitude to get ease when afflicted with abdominal pain.

To give a clear idea of what pleurisy is, the anatomy of the chest must be considered.

Persons who have seen an animal opened and the lungs removed, have noticed that those organs do not nearly fill the chest. In the living animal, however, they fill it completely, their surfaces being closely applied to its walls; the act of opening the chest, by admitting air, allows of their collapse. On the surface of the lung is a thin membrane called the pleura ; a continuation of it also lines the inside of the chest. Consequently, at each inspiration the two layers of pleura are pressed together. In health a small quantity of fluid is always found between the layers; its use is to reduce friction to a minimum. Pleurisy is inflammation of this thin lining membrane. It is usually ushered in by a shivering fit and general febrile symptoms. The pulse and breathing are both accele. rated, the latter visibly altered in character, inspiration being quicker and shorter than expiration; there is generally a short, painful cough, pain on pressure between the ribs, and great disinclination to move the fore limbs. There is no heaving of the flanks as in pneumonia ; on the contrary, the abdominal muscles are contracted to fix the chest, giving the flank a peculiar tuckedup appearance. Inflammation of a secreting structure in the first stage arrests secretion. In inflammation of the pleura there are two highly sensitive layers, abnormally dry, pressed together at each inspiration. This will explain why the inspiration is short and quick, and why a cough should be abruptly stopped, giving it such a characteristic catching sound. We can understand, too, why pain is so great from pressure on the sides, and why the animal shows such disinclination to move the fore legs.

In the second stage of inflammation of a secreting structure excessive and abnormal secretion occurs. In pleurisy, after the dry stage, an excessive quantity of fluid is discharged, and 
'water in the chest,' or hydrothorax, results. We have said that the lungs fill the entire cavity of the chest, consequently any additional quantity of fluid in the chest must diminish their capacity. This fluid in the pleural cavity has a tendency to form false membranes, or to increase, so as to fatally compress the lungs.

There is no disease to which horses are subject more quickly subdued by counter-irritants than pleurisy. Put mustard on the ribs, as advised under Pneumonia, immediately, or apply a strong liniment such as Lin. Camph. Co., or Lin. Tereb. Acet., or Lin. Sinapis Co. There are some advanced practitioners who have lost faith in counter-irritants without offering us anything better in exchange, but the rank and file of veterinary practitioners are fairly unanimous in their advocacy of vesicants, which they are convinced bring about a rapid and lasting improvement in their patients.

The treatment of pleurisy should be directed to modify the inflammation and allay pain in the first stages, then to support the system and promote absorption of fluid in the chest. Most veterinary works recommend bleeding in pleurisy. The idea is founded upon erroneous pathology, for bleeding does not check inflammation, but certainly favours exudation of fluid. A case of pleurisy should be put into an airy loose-box ; the body well clothed, and a diffusible stimulant given with a view to regulating the circulation. To open the bowels, aloes must not be used, as super-purgation is easily induced. Six ounces of linseed oil and one of spirit of nitre, or four or five ounces of magnes. sulph., is preferable. To allay pain nothing is equal to hot rugs. Dip the rug into boiling water, wring it out, and quickly apply it to the chest ; keep it in position by another rug and a roller, and as soon as cool replace by another. When the acute symptoms have passed off, the following ball may be given twice a day:-

Carb. Ammon.

$3 \mathrm{j}$

Pulv. Zingih.

jij.

P'ulv. Resine

$3 \mathrm{j}$.

Lini Far, et Theriacre .

q. s. ut ft. bol. 
It has a remarkably beneficial action on the bowels. Should the pulse remain at 50 or 55 per minute, and the breathing be laboured, considerable effusion in the chest is probably the cause; auscultation will prove the surmise. Then diuretics and stimulants are indicated. A mixture of equal parts of sp. æth. 11it. and liq. ammon. acet., given three or four times a day, is vely active; dose, half an ounce. The remedies suggested for bronchitis are generally suitable for pleurisy, but the after treatment should combine pot. nit. and iodides, as the effused material is apt to cause adhesions, and anything likely to promote absorption and excretion is of benefit :-

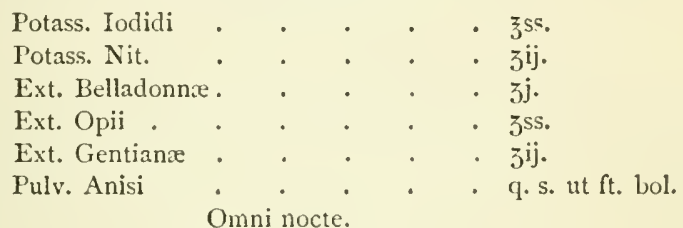

Blisters to the sides seem to promote absorption. As a last resort tapping may be tried. This is a simple operation; a small canula and trocar are thrust into the chest at the anterior border of the sixth or seventh rib; but this had better be done by a professional man. Do not starve the animal on branmash, but allow carrots, turnips, grass, \&c., with boiled linseed and barley, and a constant supply of clean cold water.

Pink Eye is a form of horse-sickness which was first called by this name in America, but is now known all over Europe as a form of influenza, and, like its congener in man, takes different forms.

The peculiar pink injection which was at first so marked a symptom has not been present in subsequent years to anything like the same extent, and, not being constant, may lead to confusion among those having but little experience of an epizootic which at one time appears as an acute attack of catarrh, at another affecting the liver, and again in other seasons displaying some depressing influence on the meninges of the spinal cord, with symptoms of paralysis or lameness, which is 
not confined to any particular limb but may affect the muscles of the loins.

With such a hydra-headed monster it is difficult to lay down any one plan of treatment, but it is essential to advise the immediate nursing of smitten animals. In nearly all cases it will be safe to give a stimulant, as follows :-

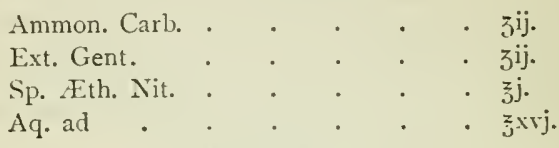

I!. ft. haust.

An eminent veterinary surgeon (the late Mr. Greares, of Manchester) used to speak of the blood-vessels of the extremities as being absolutely empty and pulseless at one period of the disease. To counteract this tendency to collapse should be the first care of the reterinarian. 'The legs should be bandaged and body clothed, the stable kept warm but well ventilated, and, after a few stimulants have been given at intervals of four hours or so, the treatment should be directed to the alleviation of any special symptoms. If the patient comes on 'blowing,' the sides of the ribs should be stimulated with mustard or an ammonia liniment.

The bowels should be regulated by small doses of mag.

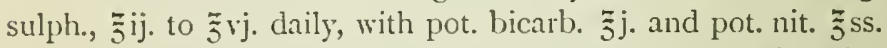

Belladonna is a favourite remedy with some vets, who give 3j. or more of extract night and morning. It may almost be said that any and every tonic should be given after the first acute symptoms have passed away. Of the many agents the Pharmacopoeia offers we prefer quinine $5 \mathrm{ij}$. to $5 \mathrm{iv}$ and extract. gent. in $\mathbf{5} \mathrm{ij}$. to $\mathbf{5} \mathrm{iv}$. doses alternately ; the latter may be given as an electuary or in solution. Balls should be aroided in all diseases accompanied by sore throat. Nustard or liniments to the throat also give relief.

The parotid and submaxillary glands often become enlarged, and have a tendency to remain so. One of the best remedies for reducing them after the disease has spent itself is I part of 
iodine to sapo mollis 20 parts, bis hebdomada-as an external application, of course.

Carrots, mashes, linseed, scalded corn, steamed hay, and everything that can moisten the food and tempt the invalid should be adopted, as loss of appetite is a common symptom. When convalescent, great care should be exercised in getting the patient to work, which should be done by slow degrees.

Broken Wind is fortunately of less frequent occurrence now than formerly, owing to the better dietary and greater air-space that horses enjoy, besides which it seldom happens that a broken-winded mare is bred from. They are very apt to be sterile when tried.

A really pronounced case of broken wind can be distinguished by anyone without a practical knowledge of the subject, yet there is scarcely any equine defect that gives rise to more swindling among 'copers' or low horse-dealers who frequent fairs and palm off useless creatures upon the unwary.

Broken wind is generally preceded by and accompanied with a characteristic cough, which the veterinarian will recognise although the flanks may have been 'stilled' by a master of the art of setting horses' wind.

The causes are generally agreed to be feeding on bulky and innutritious food, causing distension and undue pressure upon the diaphragm. The lungs, when examined post mortem, are found to have some of the air-cells ruptured into one another, besides other alterations of structure both of the lungsubstance, heart, and pericardium. The pathological condition is not so clear as to be definable in a few words; neither are the best authorities altogether in accord.

Palliative remedies are frequently in demand, and of considerable service; and of these we will mention a few of the new and of the old school, concoctions. Whatever remedies are given, it must be borne in mind that dieting is the chief consideration; the horse which is comparatively useless when fed on offal hay, and the rubbish farmers cannot sell, may be quite a useful animal in the hands of a judicious stableman, 
who will feed it on good food in small bulk and always damped, never allowing anything like an approach to distension or too much water previous to starting on a journey. Granted these conditions, a great deal of help can be afforded by the sedative agents suggested.

In a work on 'Farriery,' some two hundred years old, we find the foregoing conclusions already recognised in the following:-

'Broken-winded horses should eat sparingly of hay, which, as well as their corn, may be wetted with chamber-lye or fair water, as this will make them less craving after water.'

The same author recommends garlic — 'two or three cloves' (of garlic), 'given at a time in a feed, or three ounces bruised and boiled in a quart of milk and water, and given every other morning for a fortnight.'

We have alluded to the tricks practised by unscrupulous horsedealers for 'setting the wind ;' it is wonderful to what extent they succeed. The horse is fasted for many hours and then given large quantities of shot and tallow, bacon fat and tar, so that on the morning of sale very little motion of the flank is observed, and that may be accounted for by the seller in many ways, such as 'hot weather,' 'ridden a great many miles,' \&c. Of course, the fraud is discoiered when too late, and in a case tried not very long ago, we heard a witness, with a delicate sense of honour, specially adapted to the profession, say, ' any respectable dealer would tell the purchaser afterwards, so that he might know what to do.' 'This gentleman did not intend to 'convey the idea that the seller would teach him how to set his wind, but caution him that the horse was quite unfit to go to work.' An old remedy is the following :-

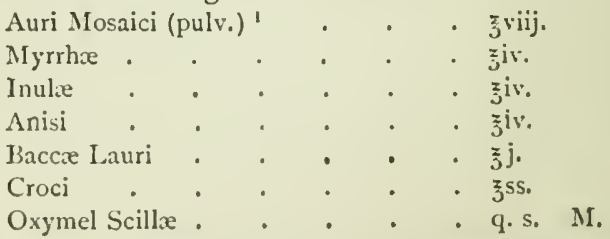

1 Aurum mosaicum consists of mercury, tin, sal-ammoniac, and sulphur, equal parts. 
The author of the foregoing recipe, having a doubt as to the efficacy of aurum mosaicum, substitutes for it a like quantity of pulv. scillæ. A ball of the size of a pullet's egg is recommended daily, or the following :--

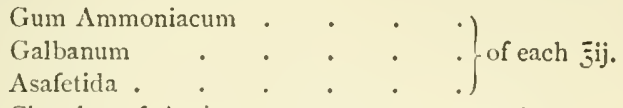

$$
\begin{aligned}
& \text { Cinnabar of Antimony . . . } \mathrm{vj}^{\mathrm{vj}} \\
& \text { Saffron . . . . . } \xi_{\text {ss. }}
\end{aligned}
$$

To be made into paste with honey, and again a quantity the size of a pullet's egg is prescribed every morning.

Modern practitioners, recognising the pathological condition of the heart and lungs in this disease, give digitalis and camphor, tar and other sedatives, of which the following is a good combination :-

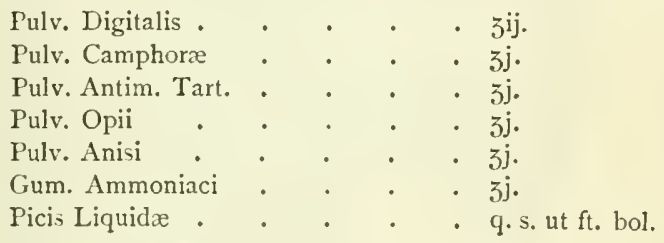

Or

Alternis noctibus.

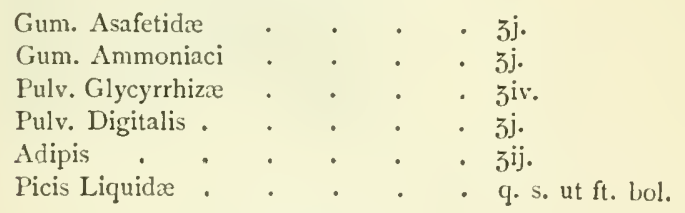

Omni nocte.

These remedies should not be persisted in when the acute symptoms have yielded, but recourse may be had to them from time to time. Any kind of fat with tar gives relief, and many horses will grow to like tar and lick it up if offered.

Roaring and Whistling are terms applied to horses which make a noise during inspiration when their breathing 
is accelerated by exercise. They represent degrees of unsoundness, as the words imply, and commonly follow upon diseases of the respiratory organs, but they are also hereditary. Either defect is a great detriment to a horse, and of course a cause of unsoundness, although some celebrated horses have won races despite the fact that they were confirmed roarers. Whistling is not so bad, but still is very objectionable, and liable to become worse. There is not much to be done in the way of treatment.

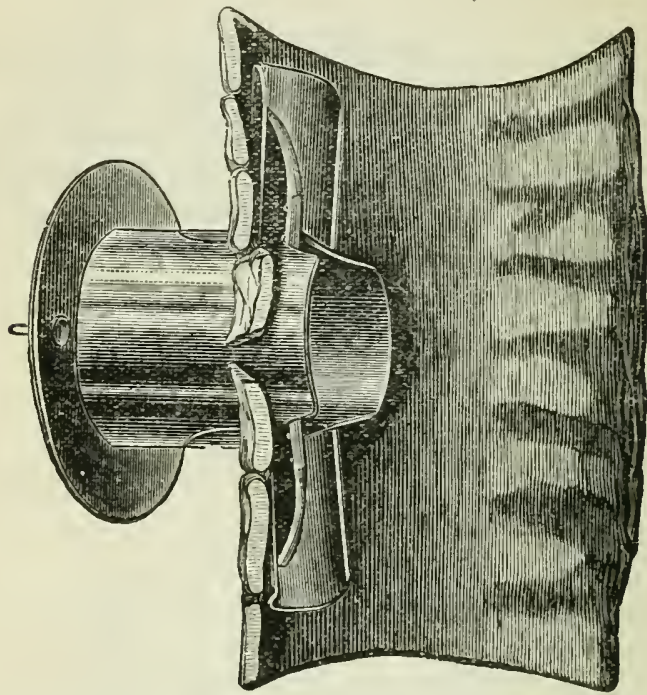

Jones's tracheotomy tube as inserted into the trachea.

The operation of tracheotomy, by which a tube is introduced into the windpipe, answers the purpose in some cases, and there are a few veterinarians who make a speciality of it. Mr. Jones, of Leicester, is perhaps the most celebrated and successful operator. These tulos are an eyesore as well as a necksore, and need careful attention on the part of the owner or attendant, since they act as foreign bodics and maintain more or less inflammation and the production of matter. 'They are therefore last resorts and are only, as a rule, adopted in the case of good 
hunters, who in other respects are valuable, but by reason of their whistling or roaring become unpleasant to ride, or so impeded in their breathing as to make the operation necessary.

Removal of one of the rocal chords and some cartilage from the larynx has been practised by some eminent surgeons as a cure for roaring, but has not been attended with sufficient success to bring it into general favour.

Congestion of the Lungs is engorgement of the pulmonary ressels-not the vessels which go to supply the lungs with nutrition, but those conveying blood into the lungs for re-oxidation. It is a result of orer-riding and driving, and not so frequently of chill or exposure. Horses that are not in 'condition' are the subjects of it. By 'condition' we mean in training for work. To 'condition' horses or prepare them for races is in these days almost a fine art. Its adepts are not perfect in the way of educational attainments and manners, but their special skill is sought for by princes and noblemen. By diet, exercise, \&c., the big gross belly is disposed of, and all the muscles of the body and limbs are made to stand out like cords and feel quite hard. The horse in hard or racing condition looks thin and 'tucked up,' as his muscular powers and heart have been developed to the highest possible pitch, even at the expense of his vegetative system, which presently demands a relaxation from the carefully sifted corn to a diet of grass, slops, \&c. This means of course letting a horse get out of condition, and has to be done from time to time with racers, who cannot be always in such high fettle, even if their legs did not fail and necessitate a holiday. Hunters are treated in somewhat the same way, being taken up from grass or straw yards in August or September, and trained, or conditioned as it is called, by degrces, so as to be ready for the hunting season. It sometimes happens that the conditioning has not been begun soon enough, or has been hurried on too fast, so that the first good run over a country finds out the animal that is not thoroughly 'fit,' and congestion of the lungs is too often the result. On reaching the stable, if not 
before, dulness is observed, the animal shivers and trembles, the legs and ears are cold, the respirations rapid, the pulse fluttering and weak, the visible membranes darkly congested, the appetite is lost, and the horse stands with his head in a corner, panting and wretched. Cough may or may not be a symptom. If the prescriber is called in at this stage, and not the next morning, he will give a powerful stimulant without delay, recognising the fact that pulmonary congestion is not at first inflammation, but stagnation, engorgement, apoplexy ; and if the heart can be roused to pump on the blood at once, all may yet be well. Alcoholic stimulants can generally be had, but can be improved upon if the pharmacy is near.

Half a pint of whisky in one pint and a half of water is not too much for a dose. Or the following :-

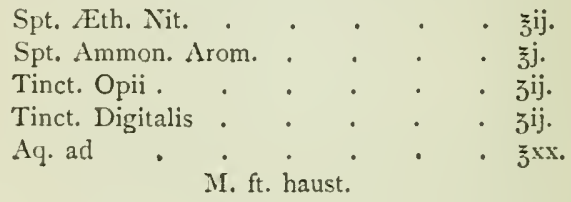

We recommend the following ball:-

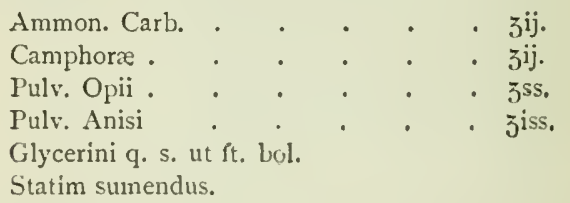

The ball may be 'washed down' with the following draught :Mist. Sp. Vini Gallici (B.P.) ․ . $\jmath^{x}$.

Mustard should be applied, as in cases of pneumonia and pleurisy, without delay, or in the case of heavy-coated or hairy subjects, lin. camph. co., or lin. camph. co. and lin. saponis partes æquales, may be applied.

The animal should have a rooniy, well-ventilated box-too cold rather than too hot; the extremities kept warm by hand. 
rubbing and bandaging the legs and pulling the ears, and clothing with hoods and rugs to induce surface warmth. If the patient does not rally, the stimulants may be repeated every four hours, or at even sloorter intervals. If the animal is going to die, the legs and ears will get colder, the flanks move more rapidly, the head hang low or be poked out, while the nostrils dilate and the increasing look of anxiety in the horse's countenance will tell its tale even to men who know nothing of pulses and pharmacopoias. The patient stands generally till he falls to rise no more, and a black bloody matter runs out of the nostrils. The lungs if examined will be found black with engorgement, and feel like liver to the touch instead of the light elastic material of healthy lung.

The cases that rally generally do so very quickly and with few of the bad results that accompany sore throats, bronchitis, or pleurisy. It is, as we have explained under Pneumonia, quite a different part of the lung apparatus which is affected. 'To prevent such accidents, condition powders, if not made of mill sweepings and rubbish, but of pot. nit. and flor. sulph. with antimony and ground seeds, help a horse very much to carry off effete material while he is being trained or conditioned, but such medicaments should not be given indiscriminately. Horses do not require drugging all the year round, but may be greatly helped by the judicious administration of medicines when making sudden changes in their mode of life, or diet. The old practice of preparing a horse for conditioning by a physic ball first, and giving him another before turning out or soiling, has experience to recommend it.

Fever.-Simple undefined or sympathetic fever occurs as a symptom of other disorders; it accompanies inflammation of internal organs, and follows severe injuries ; frequently, however, it appears as a primary affection, without any local disorder. It is customary to speak of catarrhal, gastric, and bilious fevers. These are merely cases of simple ferer, with a local complication. In one case, there is a local derangement of the air tubes ; in 
another, of the digestive apparatus, not as the cause, but as the effect of the fever.

Under the name of influenza, fever is frequently met with in the horse. Cases are generally caused by cold, run a definite course, and terminate in a quick return to health. In fever we have increased heat, a rapid pulse and breathing, with arrested excretion and secretion. In the first stages there is an irregularity in the circulation; this is as far as a number of cases ever get, as a good stimulant and a little care produce a healthy reaction.

Stock fever draughts should contain an alcoholic or ammoniacal stimulant, a sedative, and a tonic. The effect of alcohol, after the primary diffusion of blood to the surface, in itself a desirable property, is to reduce the bodily heat. Ammonia or digitalis, by increasing the force and diminishing the frequency of the heart's contractions, helps in the same direction, and some bitter tonic carries on or sustains the good work; hence the esteem in which a good 'fever drink' is held by practical horsemen, who know when to give them. 'This is a good formula :-

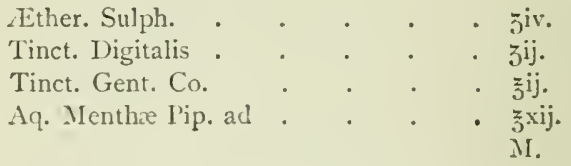

and this :-

Sp. Ammon. Arom.

Tinct. Card. Co.

'Tinct. Hyoscyam.

sp. Nither. Nit. .

Aq. Chloroformi ad

$$
\begin{aligned}
& \text { - } 3 v j . \\
& \text { - 3vj. } \\
& \text { - } 3^{i v .} \\
& \text { - } \tilde{j} \text { j. } \\
& \text { รxw. }
\end{aligned}
$$

Care should be taken not to give an ordinary dose of aloes in riolent fever, as super-purgation frequently results. Four ounces of linseed oil or sulphate of magnesia is preferable; or good effects may be relied on by giving carbonate of am. monia in two-drachm doses twice a day. 'The body should be well clothed; fresh air and cold water allowed ad lib. 
Green food and boiled larlcy and linseed are much better than a continuance of bran mashes, which only act as a laxative from being indigestible, and so do not support the system.

Mud Fever.-The disease known among lorse-owners as mud fever is more or less of a regular visitor in winter time both in town and country districts, and the conditions which bring it about are not very clear. It is more frequently the scourge of the gentleman's than of the poor man's stable; while most diseases find a home among the ill-cared-for and ungroomed, this is an exception, and it was as if by accident that the remedy, or rather preventive, was discovered.

In i 87 I many London horses died, and so aggravated a form did the disease take that many firms were unable to horse their vehicles, and great inconvenience and loss resulted. Some one then observed that night-cabbers and other horses that are put away dirty escaped, while the carefully washed and bandaged hunter was laid up. It was found that when the hair was left on the lower parts of the limbs, and the dirt was left on the hair till $d r y$, very few cases of mud ferer appeared; since that time the custom of clipping down to the elbows and nearly to the hocks has become much more fashionable, and is adopted by many without knowing the reason. Still, with all precautions, mud fever is met with every winter. Metropolitan 'vets' say the tram companies are to a great extent responsible, as they put down salt to make the track clear when frosted, and the salt increases the irritating power of the mud. 'This may be so; but horses suffer that have never been in a town at all, and on chalky and clay soil alike, so that many horsemen think the disease comes from within, and is but a local manifestation of a sysiemic condition.

The horse, be he carriage, hunter, or other breed, comes in all plastered with mud, and is washed, dried (more or less), and put away for the night; if badly attacked he will be feverish and off his feed in the morning, stiff behind, and sore to the touch all down the front of the hind legs, and often all along the belly; the hair comes off very quickly, and the 
outer skin, or cuticle, desquamates to such an extent as to leave a raw surface and great tenderness. Sometimes there is great swelling of the hind legs, the front ones participating to a lesser degree, and the belly often much swollen right along to the posterior portion of the sternum. In very bad cases the whole surface assumes a confluent condition, and the constitutional symptoms are proportionately severe, the thermometer registers $104^{\circ}$ to $105^{\circ}$, and the animal rapidly becomes emaciated.

The treatment will be directed to reduce the pain and irritation, and prevent the malignant condition above described; to lower the temperature and restore appetite, and make the horse sufficiently comfortable and supple in his limbs to lie down on a good bed. First, foment with a lotion composed of glycerini Oss., aquæ $\mathrm{Cj}$., at a temperature of about $\mathrm{J}_{0} \mathrm{~F}$.; and having with this softened off-and not rubbed-any adhering dirt or serosity, carefully dry the parts with a very soft towel, or lint, and apply one of the following lotions :-

Acidi Carbolici . . . . . $3 \mathrm{j}$.

Glycerini • . . . . . $\overline{3}_{\mathrm{ij}}$.

Aquæ ad . . . . . . Oj.

II. ft. lotio. Ter die utend.

Acidi Carbolici . . . . . $3 \mathrm{j}$.

Ol. Sesame • • • .

M. ft. lotio. Applic. omni die.

Liq. Plumbi Subacet. .

O1. Lini

M. ft. lotio. Omni die.

All the foregoing are well tried and old friends of good veterinary surgeons. The last named is much favoured in the south of London in ordinary cases, and the carbolic form when there is a tendency on the part of the skin to slough.

The benefit of bathing the inflamed parts with very much diluted glycerine can le scarcely credited by those who have not used it, and we know of a celebrated lotion which is com- 
posed of this only, with a little liquor rosæ, with elaborate directions as to the use of rain-water with it, and so on.

As regards internal remedies. If the fever runs high, doses of 15 to 30 minims of tincture of aconite (B.P.), with $3 \mathrm{ij}$. chloral hydras or pot. bromid. $\tilde{5}$ ss., bis die. If there is constipation linseed mashes or tea should be ordered, but no aloes given, or other purgatives stronger than mag. sulph., ziv. c. p. fœenug., $\tilde{3} \mathrm{j}$, or other spice, to induce the horse to eat it. With the abatement of fever small doses of tinct. opii and spt. æth. nit., $\tilde{5}$ ss. of each, may be given ; or, if a ball be preferred, give pulv. opii $\exists_{\mathrm{ij} .,}$ ammon. carb. $5 \mathrm{j}$., lini farin. q. s. ut ft. bolus, bis die. A little daily exercise should be enjoined, however stiff and unwilling the horse may be to move. Carrots, bran mashes, \&c., in small quantities to tempt the appetite and regulate the bowels, and on returning to work a good coating of the liq. plumbi and oil lotion; some tonic powders in the daily food will help to get the horse in condition again. The following is a good recipe :-

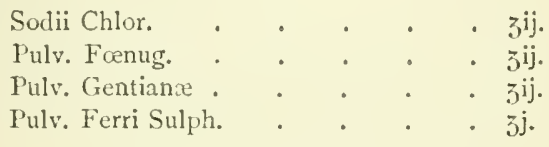

M. ft. pulv. Nocte maneque c. cibo.

To prevent a recurrence of the disease, the legs should not be clipped, and the mud should be allowed to dry and then brushed off. Washing should be strictly prohibited.

Strangles.-A disease of colthood. The chief symptom is a swelling under the jaw, which increases until the formation of pus and the pressure within make a thin place where the abscess is said to 'point.' There is often a great amount of constitutional disturbance and high temperature. Febris pyogenica is the scientific name.

Treatment consists in forwarding the pus formation and inducing it to come to the surface. When a soft place or 
prominent one is felt the lancet is to be boldly plunged into the abscess and the matter liberated. It is often very thick

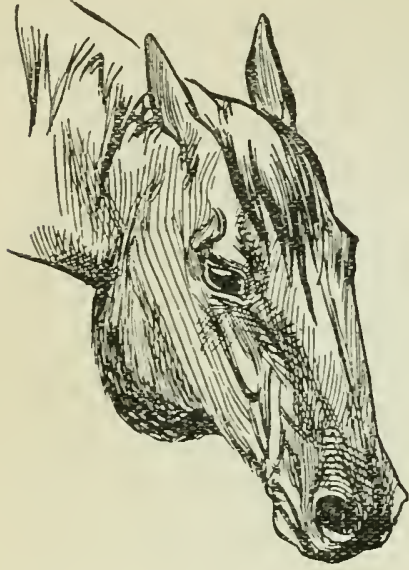

Strangles : showing the swelling under the jaw. and may need some manipulation to get it out. The introduction of a little ol. tereb. and ol. olivæ, partes æquales, will facilitate the discharge, after which it commonly heals with great rapidity. Poulticing is seldom practicable, as the disease occurs most often with youngsters at grass, and it is better to prescribe a stimulating liniment such as lin. alb. or saponis, and advise trough feeding with soft foods, like linseed and bran mashes. A tonic of iron and gentian is found very useful in restoring strength when the pus has been liberated, the disease being very debilitating and sometimes causing death from rupture into the chest of secondary formations.

Bastard Strangles is a term sometimes applied where multiple abscesses form, and by others it is used to describe inflamed glands and catarrhal symptoms in seasoned horses, which often threaten to develop into genuine strangles. Professor Williams gives the term to that more severe form of strangles in which multiple abscesses are formed and other glands often affected besides those in the sub-maxillary space. Notwithstanding careful treatment colts sometimes die of strangles from the bursting of internal abscesses; in some scasons a more malignant type of the disease prevails, and although it is a moot question as to its infectivity; there is a characteristic benignity in the symptoms some years as compared with others. Inoculation and reproduction of abscess with pus is not sufficient proof of infection, as the introduction of any foreign material may produce an abscess. 
Treatment.-The great object to be aimed at is to get the abscess to 'point,' and any and every remedy calculated to produce such a result may be used. If the convenience exists for constant hot poulticing, by means of a bag of bran tied to a head collar, it should be done, at the same time introducing a dram or two of ung. resine into each poultice. If the animal is wild or there is reason to suppose poulticing will not be properly carried out, it is better to prescribe a liniment of ol. tereb. rub. and ol. sesamæ, p. æ., and repeating the first brisk rubbing in the course of a few days if the abscess has not broken or is not ready to lance; its ripeness can be pretty easily determined by pressing the thumb or finger over the most prominent part, when it will feel as though only the skin or a very thin partition remains between the contained fluid and the parts being pressed upon.

An opening an inch or more long should be made, and gentle pressure exerted to remove the ropes and clots of pus which sometimes, but not always, form, and are a source of further trouble if the abscess is not fully eracuated. The further treatment of the wound consists in draining by the insertion of a wisp of tow dressed with the liniment above mentioned. As this disease rapidly undermines the animal's strength, and in the case of colts arrests their growth, it is advisable to give tonics, and supply tempting and nutritious food, such as carrots, scalded oats, soaked peas, and greenmeat if obtainable.

A tonic powder will, in the majority of cases, be most convenient to administer, and the following is seldom 'nosed' over and neglected :-

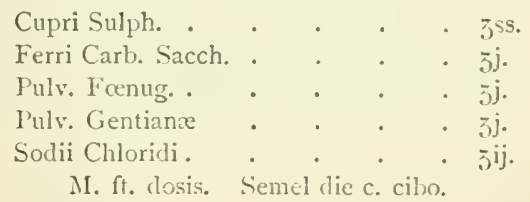

Light diet, gentle exercise, regular grooming, demulcent drinks, such as barley-water or water mixed with honey and powdered marshmallow or liquorice, are accessories to the treatment. 
The animal must be kept in a warm place, and covered with a cloth. Give no drink cold, but mix it with barley-meal and honey; for food, give clover mixed with barley-meal and moistened with water.

Shivering.-This term does not apply to rigors or the shivering fits which usher in febrile complaints, but it is used to denote a serious affection of the spinal cord. It is the analogue of chorea in dogs, and comes on during colthood almost always, though similar symptoms are known to follow injuries to the spine and the meningitis of influenza. It is nearly always incurable, because due to some lesion of the spinal cord. The symptoms often do not display themselves until a horse is required to back a load or keep one back in coming downhill, but in bad cases horses will stand still and shudder or shiver, with a sort of general spasm of the body and rigidity of the limbs. It passes away again quickly, but such animals are unsound and sooner or later become incapable of lying down and finally of getting up when they fall down in sleep.

Megrims.-The pathology of this most dangerous disease of the horse is not very clearly made out. Some hold the theory that the fit is caused by an apoplectic condition of the brain, while others suggest anæmia of that organ as the cause. In its manifestation it more nearly resembles epilepsy than anything else.

There is nothing in the examination of a horse that will enable a veterinary surgeon to diagnose the subject of megrims. He will appear to be perfectly well, even if sold on a short trial ; but one day the fit will come on when he has been out for some time ; he stops suddenly, shakes his head, sways his body, and even falls down in bad cases, but the fit does not usually last long, and if nothing is loroken he will resume his journey apparently only dull and depressed in manner. It is commonly harness horses that suffer from this disease; and warm weather, uphill work, and tight collars contribute to bring on a fit. $\Lambda$ 'piped' collar should be used. 
Bleeding from the palate, aloetic purges, and bran mashes are commonly recommended; but a horse subject to megrims is a dangerous creature, and the owner will save himself loss by an early sale-to a stranger.

Sore Throat.-Acute inflammation of the throat sometimes causes suffocation, when it arises from an alsscess formed in the vicinity of the throat at the back of the tongue, as in strangles. Do not bleed on any account. Linseed poultices should be placed on the throat; barley-water, sweetened or acidulated, should be given as a drink or a gargle. Milk diet should be provided without delay. The following is another method of treatment :-Make an electuary of I part each of nitre and sal-ammoniac, and 2 of Glauber's salt with water and meal. Smear on the tongue five or six times daily a quantity twice the size of an egg. Rub the swollen parts three or four times daily with a mixture of 2 parts each of mercury and marshmallow ointments with I part of camphor ointment. Protect from chills, and give only warm water to drink.

Stomach Staggers, or Grass Staggers, is an affection of the brain caused by impaction of the stomach. It is commonest among young animals at grass in the autumn.

Symptoms.-Dulness, disinclination to more, fulness of the abdomen, perhaps distension and constipation ; breathing slow and heavy, pulse slower than usual, 30 to 35 a minute instead of 40. It comes on suddenly, and soon ends in death or recovery.

Treatment.-If discovered in time a brisk aperient is usually effective, but bleeding from the jugular vein is even more rapid in removing the brain pressure, for it is in the cerebral vessels that the trouble is displayed, though primarily caused by engorgement of the stomach and bowels.

Sleepy Staggers, a similar disease, comes on gradually, runs a slower course, and is not attended by the marked fulness and constipation of stomach staggers. 
Treatment.-As the stomach is packed with food, balls and powders can hardly be assimilated. Strong solutions should therefore be given. The horse cannot vomit, so that reilef must be obtained through the bowels. Give several doses of mag. sulph. in a good deal of water. Abstinence from food for some time must be enforced, and when again fed it should be from a manger or rack. Horses liable to staggers should never be turned out to grass, as the pendent head is provocative of a return. Salines are especially useful in combination with one or two small doses of aloes, as the latter in small doses is diuretic, while salines by osmosis more rapidly deplete the system than anything. Bleeding is recommended to relieve the acute symptoms in the first place-the saline takes up the running and prevents the increase of temperature and blood-making which follows blood-letting-a circumstance which renders bleeding so seldom desirable except in certain acute cases.

\section{Cystitis-Inflammation of the Bladder.-Symptoms.} -Cording of back, straddling of the hind legs and posturing for urination without result, and, in the case of males, frequent partial unsheathing of penis; thirst, movements of the tail; the horse turns to look at its flanks. A high degree of fever accompanies the malady, quick, small, irritable pulse, and distressed countenance. There is an indisposition to lie down though rest is needed. This disease is liable to be mistaken for laminitis, as the animal's behaviour is very similar when the hind feet are affected, arching the back and standing ' all of a heap as though marle in one piece.'

Treatment.-Clysters act as internal poultices and should be frequently thrown up the rectum. They may contain glycerine and some cxtract of belladomna. For internal use, too, there is no other drug which acts so well upon the urinary organs when inflamed. A first bold dose of an ounce of the tincture may be given and followed up three times daily with twodrachm doses, regulating the amount and continuance not only by the condition of the patient but the effect upon the pupil 
of the eye. If the latter is found much dilated and unable to contract in the presence of a strong light, the medicine must be stopped. Give mucilaginous drinks and clysters, and apply a poultice of boiled bran to the loins. The bladder may be emptied by means of a catheter, an operation which requires experience. As the patient improves give bitter decoctions with nitre. When the inflammation is due to stone in the bladder, this can only be removed by operation.

Eczema.-The skin diseases of the horse commonly called eczema are, in reality, distinct affections, but having in common the production of minute resicles which break, and by coalescing form a serous mass, or scabs. It is not contagious, but often occurs upon several animals in the same stable from an error of diet in which all have shared.

Treatment.-Change of food, especially to green-meat and carrots, washing with soap and dressing the sore places with ung. zinci or ol. carbol. I in 25 , or acid. carbol. I part, glycerin. 2 parts, aq. 20 parts, with a few alterative powders, will generally effect a cure. Half an ounce of pot. bicarb. and pot. nit. $5 \mathrm{j}$. in the drinking-water night and morning is also beneficial. Where balls are preferred, either of the recipes for alteratives may be used with advantage.

Mallenders and Sallenders. $-A$ skin trouble which comes in the flexures of the knee and hock (behind the former and in front of the latter; see illustration, p. 60) is often described as a form of eczema; the horseman's name being 'mallenders' when affecting the fore limb, and 'sallenders' if a hind one is implicated. This affection is really on a par with cracked heels (see p. I 25 ), the parts being provided with more or less glandular structure, whose office is to keep the skin soft and unctuous where friction would arise from the bending of knees and hocks. When, from the irritation of dust or mud, these are inflamed, we get the results described under cracked heel. The disorder would seem to be associated with some constitutional disturbance, and become chronic, and, 
for this reason perhaps, has been classed as eczematous. We have known horses with whom the discharge seemed to have become necessary, as in the case of ulcerated legs in human beings, drying up the mallender or sallender being followed by some congestion of the foot and more or less lameness.

Treatment.-An aloetic ball, where circumstances permit, followed by alterative powders in which pot. nit. or pulv. resinæ has a prominent place. As an ointment, hamamelis and vaseline or ung. zinci ox. with acid. carbol. $m x$ ad unciam. Quite recent cases may be dried up and lastingly cured by a more astringent ointment, as alum. exsic. $3 \mathrm{j}$. to mel. exot. $\overline{3} \mathrm{j}$.

Pityriasis.-Is a condition of the skin resembling bransmall scales without a visible vesicle or other antecedent eruption form in great quantities and produce a very unsightly condition. It is often caused by poverty, and both horses and cattle are affected with it at the end of the winter.

Treatment.-Change of food, alteratives, and oily dressings. It is said to be the result of incomplete oxidation of the carbonaceous principles of the food, and hence it is advisable that all starchy and sugary foods should be withheld.

Prurigo or Pruritus is a scientific term for an irritation of the skin that cannot lee made to fit in any of the squares which an exact system of nomenclature would design for it. In veterinary practice such cases are frequently met with, and no cause can be assigned, and too often no remedy be found. We use a good many long words in connection with skin diseases, but our knowledge of dermatology is very meagre, and the treatment empirical. If no definite disease is to be found to account for a violent itching, and no parasite can be accused of the mischief, it is well to give remedies that have a distinct action on the skin, like antimony, sulphur, pot. nit., \&c., and apply a sedative lotion as follows :-

Liq.. Potassa. . . . . . $3 \mathrm{j}$.

Acid. Hydrocyanici dil. . . . . sss.

Aque ad

M. ft. lotio. sipe utend. 
$A$ lotion that has sometimes succeeded when all others have failed consists of :-

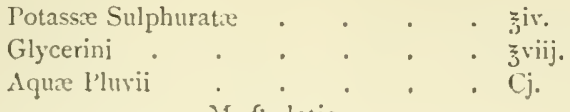

M. fi, lotic.

The animal's skin is previously prepared for this application by washing in an alkaline water such as a packet of Hudson's Extract of Soap in a bucket of warm water. Partially cleared with a scraper and the lotion brushed on with an ordinary water brush.

Itchy Tail.-This is a form of prurigo which spoils the appearance of many a horse, and he who prescribes successfully for it will earn the gratitude of both master and servant. No doubt in many cases it is nothing more than a vicious habit, but it had its origin in some irritation of the tail or the rectum, and often ceases when the latter has been cleared of fundament bots or of those small round worms which haunt the terminal portion of the intestinal canal. Treatment should not be despaired of until the prescriber is quite assured that no parasites are present. We have known many cases relieved for a considerable time by first washing the tail thoroughly with soft soap and abundance of water, and then applying the above lotion.

Many remedies have been suggested for bots, but they come away in their own time, and are perfectly indifferent to immersion in ol. terebinth. and other reputed vermifuges.

Acne or German Measles in the horse is a pustule which occurs in the mane and occasionally in the tail; the rubbing of the collar either produces or farours the site for the parasite which causes it. It works its own cure when it breaks, but a little ung. resinæ favours the healing process. The collar should be kept off the place, and this may be done oftentimes by tying the harness to the saddle pad, since it is in 
going downhill that the collar jolts upon the mane. It is sometimes necessary to give the horse a holiday for a few days.

Of late years there has been a very troublesome form of this malady, known among job masters as German measles because introduced from the Continent. It begins under the harness, but often spreads along the back and over the poll, the withers, shoulders, tail, \&c., making the horse very unsightly and causing much annoyance.

Treatment.-The best of all remedies is that of sweating in several rugs while calling upon the animal for severe exertion; dressing with a cresol ointment while still wet, and the daily administration of flor. sulph. in doses of an ounce or more. This exhales through the skin and makes the host untenable after a time.

Surfeit, or Nettle Rash, is a name given to an eruption of the horse's skin which is not parasitic or contagious, but appears to arise from acute indigestion, from chills, drinking cold water when hot, \&c., and generally occurs when the coat is being changed. In the form known as urticaria it will sometimes appear in a few minutes, lumps coming up the size of a hazel-nut along the neck, shoulders, and sides, and disappearing again almost as quickly. The more common form of it is not so sudden either in coming or going, but the lumps are hot and irritable, and the outer skin or cuticle desquamates and leares scaly semi-bald spots, or else the growth of white hairs.

An aloetic purge of from 4 to $S$ drachms should be given, if the horse can be spared for two or three days from work, or alteratives in the case of a seven-day cabber. The alterative powders in common use are sufficient-sulphur and nitre affecting the skin very quickly and carrying off effete material by the diuretic action of the latter. It may here be remarked that vicarious function is more readily excited in horses than other animals, and even in health the urine of the horse is often loaded with material which precipitates immediately on contact with the cool air. 
If the horse is disposed to bite himself (or his clothing) and rubs the stall-posts, it is well to apply the following lotion :-

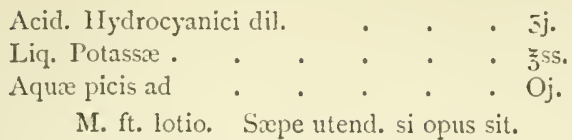

Worms in Horses. - The intestines of the horse are infested by three or four different kinds of worms, of which tapeworms are rarest, and ascarides, or round worms, commonest. These latter are usually located in the stomach and small intestines. When in large quantities they are roided at times with the freces, and this, and the poor condition of the animal, are the two symptoms of their presence. The absence of worms in the dung is not proof positive that an animal is free from them. In this case we can only diagnose negatively, by the poor condition and absence of any other cause. The most effectual remedy is a good dose of aloes, given on an empty stomach. This effectually removes the parasites, but as the patient is probably weak a little tonic medicine is required, a very good form in such cases being-

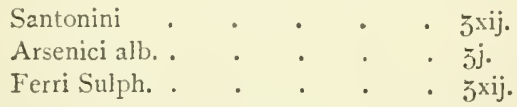

made into twelve powders, and one given in the corn every day ; or we may give half the powders and then the physic, and then the other six.

As a matter of tact, it will be found best to give the powders as a worm powders, and advise a dose of physic after, and this because horsemen have a sort of idea that worm medicine is only correct in the form of powders. The tapeworm of the horse is only small, and the joints might easily pass out in the dung unobserved. The best remedy is ol. filicis maris, in doses of two or three drachms.

It is unfortunately true that nothing is more uncertain in veterinary practice than worm medicines. The same ball is 
sworn by in one stable, and at in another. Like fish sometimes the worms will bite, and at others they are off their feed, notwithstanding all the fasting on the one hand or ground-baiting on the other. Some practitioners give 'ground bait' in the form of mashes, and others prescribe abstinence; but these tricks are often as worthless as fishing from the opposite bank when sport fails on the first side. The only thing to do is to give those remedies that are rough on worms, and, failing on one occasion, to try, try, try again, and patience will generally be rewarded at last.

It is a matter of common observation that the large round worms of the horse (Ascaris megalocephala) come away freely in May and June, when horses first get a cut green-meat. If the dose of aloes already recommended should not be effectual, the following may be tried :-

\begin{tabular}{|c|c|c|c|}
\hline Santonini & - & . & . \\
\hline Pulv. Vitrei & . & . & . \\
\hline Ant. Tart. & . & & \\
\hline Aloes Barb. & & • & - \\
\hline Ferri Sulph. & Exsic & & . \\
\hline $\begin{array}{l}\text { Alum. Exsic. } \\
\text { Excipient. q. }\end{array}$ & & & \\
\hline
\end{tabular}

Bol. j. bis hebdomada.

This may be given in powder with the exception of the aloes and alum. exsic., though some horses will take them.

If the remedies given should fail, the rendor had better be slow to pooh-pooh the presence of worms, or it may happen that some carter with the boldness of ignorance will afterwards give arsenic $5 \mathrm{j}$., c. antirn. tart. $5 \mathrm{jj}$., and bring round half a bucketful of ascarides to show what he can do when he chooses. We should not recommend this remedy, though often successful, as we have seen ulcerated bowels, causing death, afterwards - a long time afterwards, when no one would believe the real cause of $\mathrm{jt}$, and least of all the carter who has 'taken you down a peg' over the worms.

It is not the large round worm that, as a rule, does so much harm to horses, but the palisade worms (Strongylus 
Birmatus and tetracanthus), that infest the mucous lining and make nests in it; these and some of the strongyles are im. possible to cast out, as they bore through the intestines and make nests on the junctures of blood-ressels, returning to the intestines to feed when arrived at a certain stage. We have known scarecrows of horses from this cause upon whom enough money has been spent in anthelmintic medicines to buy a better horse, but they have never been cured; the adults liave been constantly expelled when on their feedinggrounds, lout the young keep taking their places, and 'the cry is still, they come.'

Bots are the larve of a gad-fly (Estrus equi). The eggs of this fly are deposited upon the legs, \&c., of the horse, and

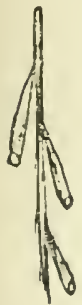

Eggs of Bot Fly on Horse's Hair (\$lagnified).

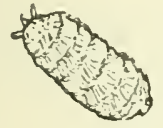

Fundament or Red Bot.

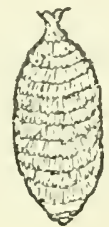

Stomach Eot.

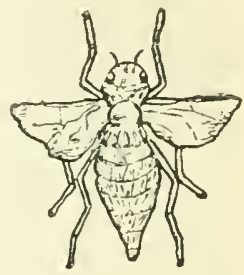

Female Bot Fij.

are taken into the stomach by the horse licking himself. The larræe escape from the eggs, and fix themselves to the mucous lining of the stomach, where they remain during the winter. The following spring they let go their hold, and are removed along with the frees.

Another variety, known as the 'fundament' bot, is found just within the anus, the fly depositing its eggs while the horse at grass is in the act of defacation and the folds of membrane are momentarily exposed. The presence of these is often the origin of itchy tail (see p. Iog). They are best got rid of by hand picking-an operation both tedious and unattractive, 
but the results are worth the trouble. It is thought by some that the introduction of ung. hydrarg. is inimical to their existence, and it is as well to provide the stableman with some of it, as he will the more likely persevere in the use of his fingers if supplied with an unguent calculated to make the parasites loose their hold. No medicine will totally destroy stomach bots, but a very useful draught is $\mathbf{z} \mathrm{ij}$. of ol. terebinth. given in ol. lini $\mathrm{Oj}$.

Glanders is the greatest scourge known in the equine world. It is as old probably as civilisation, and was described 2,000 years ago by Hippocrates. Acres of paper and rivers of ink have been consumed in its discussion, and the midnight oil has been burned by learned professors and zealous students cultivating the 'specific bacillus' of glanders. There are two forms of glanders - the acute, and chronic or subacute-and it was of course the latter kind that the old-fashioned veterinary surgeons treated medically. Of farcy and water farcy so called we shall presently have occasion to speak.

As glanders may not be treated by chemists, and its diagnosis must be left to the duly qualified veterinary surgeon, we need not here discuss it save to enumerate some of its prominent symptoms and warn readers against giving balls or handling the nostrils of suspected horses, since the disease is transmissible to man, and several veterinarians and horsemen have lost their lives by it. It is an infectious disease, and always present in some large cities with o!d and insanitary stables.

'The symptoms of glanders are chicfly: Discharge from one or loth nostrils, frequently onc only, and that the lcft-at first watery, and scldom recognised as of any importance, afterwards becoming glutinous and lastly pus-like ; loss of condition, staring coat, inappetence, glandular swelling under the jaw, cough, scalded nostrils, ulceration of the lining membrarie of the nose, lumps upon the skin, most often on the inside of the hind legs, front legs, and about the head in the order in which we have placed them. Some or all of these symptoms 
may exist, and a veterinary surgeon should always loc consulted in any case the least suspicious.

The Mallein test. $-\Lambda$ substance termed 'Mallein,' which is an extract of the products of the bacillus of glanders ( $B$. Mallei), is now extensively employed. It is injected subcutaneously and (in the case of glandered animals) causes a rise of temperature and characteristic swelling. This substance has proved of the utmost diagnostic value, but only an expert can appreciate the exact value of the reaction or certify to the authoritics.

It should be clearly understood that no treatment is lawful, but that notice is required to be given where a horse is clinically. affected.

Farcy is generally described in veterinary works before glanders, as it is too often the precursor of it. So much so and such a malignant form does it take that the Veterinary Department of the Privy Council place it in the same category, and require animals so affected to be destroyed. Teterinary surgeons are now umanimous as regards glanders and farcy being one and the same disease, and due to the same microorganism, viz., Bacillus Mallei.

The counter prescriber should very clearly understand that he ought not to be a party to the treatment or keeping alive of an animal affected with farcy. 'The Privy Council has lately (1899) issued very stringent orders in this connection, particulars of which can be had from the Agricultural Department. It is important to call attention here to the fact that most horse owners persist in calling a swelled leg by the name of furcy, or 'water farcy,' or 'fassy.' 'This malady is perfectly amenable to treatment and is not in any way related to farcy or glanders.

Water Farcy is often confounded with farcy. It has no relationship whatever to the latter, is rarely fatal by itself, and may generally be cured in a few days, or at most in a week or two, but is liable to recur. It is an inflammation of the lymphatics (J'mphangitis), due to irritation of rich food when 
the animal is not working, and hence its liability to occur after the animal has been standing in the stable during the weekend and having the same amount of food as if it had been working. It is consequently frequently called 'Monday morning disease,' and is also known as 'Weed,' 'Shivers,' 'Shakes,' and by a variety of other names. Cart-horses and heary or crossbred animals are common subjects. Its appearance is sudden, and usually ushered in by a rigor (although this is frequently overlooked); the horse may be apparently well overnight and unable to move across the stall in the morning; the leg-for it more often affects one leg than two, and usually the off hind - is very much swollen, hot, tender, and stiff; the swelling too has a more or less defined line, sometimes looking as though a cord had been drawn tightly round the thigh, differing in this respect very much from a farcy leg, which does not pit on pressure as this does; there are no nodules or buds here, though surfeit bumps may coexist and deceive the unwary. It is a common thing to find healthy carthorses of the hairy-legged variety with bunchy legs, but it is not farcy.

The medicinal treatment most effectual must be directed to 'calling upon those emunctories the kidneys' and we may say they should be called on by a variety of visitors to get the needed response.

Aloes is known to ereryone as a purgative in the form of a physic ball, but it is too often forgotten that it is one of the best diuretics, especially when given in solution or in small doses in form of a ball. Of pot. nit. and pulv. resinæ we may require assistance, but in the first stage of big-leg or water farcy, or whatever else we may choose to call it, there is no beating the following draught, unless constipation exists to an alarming extent and necessitates a purge.

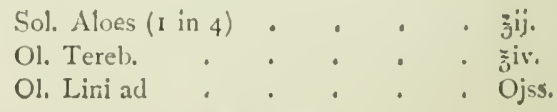


This may seem a bold dose of turpentine, but we have known more than that given to a pony with good effect, and we are now supposing a cart-horse to be the subject of our remarks. This should not be repeated, but the local symptoms should be relieved by fomentation with hot water, into a gallon of which should be poured-

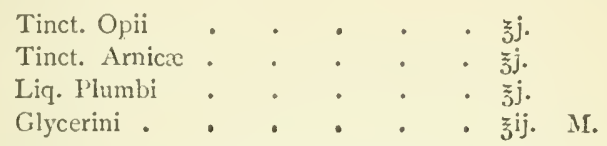

After which the limb should be encased in woollen bandages put on moderately tight. Diuretics should be continued, as well as the fomentation, for several days, and exercise enjoined as soon as possible, or ' rather sooner,' as the difficulty of getting the horse to move at first should not prevent the attempt. A loose box, if possible, should be obtained, and every encouragement given to lie down by the provision of an ample bed. As a subsequent diuretic we recommend--

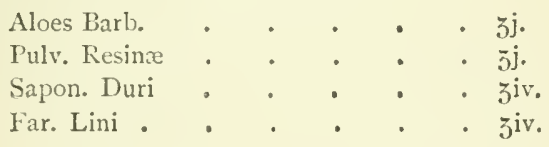

Excipient. q. s. ut ft. bol. Omni nocte,

Water farcy sometimes affects a front leg and one half the surface below the breast-bone, but it has the same defined line and doughy feel whether in one or both hind legs or forward.

Where there is reason to suppose that fomentation will be but indifferently carried out, we would substitute for it-

$$
\begin{aligned}
& \text { Liq. Plumbi • • • • • } \tilde{5} \text { j. } \\
& \text { Ol. Sesamre • • • • • Oj. }
\end{aligned}
$$

Apply this all over the swollen surface; it gives much relief. The horse should have bran mashes only until the inflammar tion has subsided. 


\section{Diabetes-Polyuria-Frequent Staling.-Under the} above names a disease of the horse often calls for attention. It is not true diabetes as met with in the human subject, as sugar is not present in the urine. It is characterised by extraordinarily intense thirst, and by the evacuation of urine to an abnormal extent. This has been known to amount to from ten to fifteen quarts daily. The animal loses appetite and rapidly becomes weak and emaciated. The condition is readily and most effectually treated by iodine, and the following formula is given by Finlay Dun, on the authority of Mr. Dollar, as having proved in his experience superior to other combinations more correct chemically.

$$
\begin{aligned}
& \text { Iodini } \\
& \text { Ferri Sulphatis : } \quad \cdot \quad \cdot \quad \cdot \quad \cdot \quad \cdot \quad \cdot 3^{\text {ss. }} \\
& \text { Pulv. Gentiane }
\end{aligned}
$$

Make into a bolus, and give one daily. In very bad cases a dose may be given night and morning, but ordinarily once a day is sufficient. About six doses will generally effect a complete cure. The food should also be inspected, as it is well known that the condition is frequently caused by musty hay, kiln-dried oats, \&.c.

The iodine may be combined with pot. iod, in such a ball as this :-

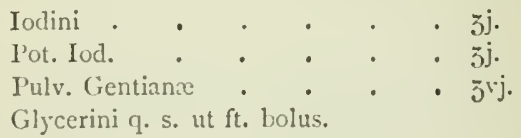

One to be given night and morning.

Another ball which appears to have a beneficial effect is composed of :-

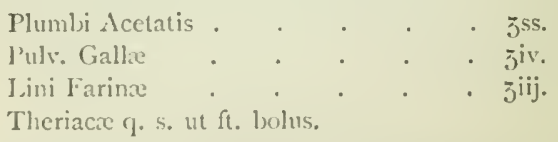

One of these balls to be given every night. The iodine balls should be freshly prepared, and wrapped (but not sealed) in paraffin paper. 


\section{LAMENESS IN HORSES}

OxE of our most eminent veterinary surgeons has been heard to say that half a ret.'s living is got below the horse's knees and hocks. If this statement be not strictly accurate, it serves to illustrate the importance of the subject. The principal causes of lameness are fractures, sprains, ring-bones and sidebones, splints, spavin, pricks in shoeing, corns, canker, thrush, sand-crack, seedy-toe, false quarter, quittor.

To the hock may be attributed by far the greater number of hind-limb lamenesses, though many stablemen believe horses to be lame in the 'round bone,' by which they sometimes mean the stifle-joint (or patella-bone), and sometimes the haunch or hip. We knew a farrier who, when in doubt, used to blister all the lot, on the principle of a larger net catching more fish than a small one.

Lameness caused by injuries to the hip and stifle are not very frequent, and cannot generally be diagnosed by any but an expert veterinary surgeon; when, however, this lameness is known to be caused from a fall or blow upon the hip or upon the stifle-joint, treatment may be adopted such as would apply to any other direct injury, and failing an early recovery a blister charge may be applied, and Dr. Time called in to complete the cure-valuable allies are time and the r'is medicatrix natura. If few of us would like to confess to our clients how much we are indebted to these, we should not forget them in the case of obscure and obstinate lame cases. We have known lame horses get sound again and prove good servants after a rest of eighteen months and even two years, which to a farmer is not so great 
a loss, though to a town horse-owner the expense is ruinous and not to be entertained. Some farmers do a good business by buying broken-down omnibus and tram-car horses and keeping them at grass till restored. One farmer we know uses some marsh-land where are many leeches, and does nothing but turn out crooked and 'stale' horses for the leeches to make sound again.

Some lamenesses are caused by injuries to the spine, 'over the bridge' as horse-dealers say, and may be due to degeneration of the spinal cord or 'chinked back,' which is generally intended to mean some injury to the spine-bones, which makes a horse unable to back or turn or support a heavy load downhill; to a disease of colthood known as 'shivering' and due to some spinal degeneration but little understood (see p. 104). It is often of such a nature as not to be observed till the animal is put to work, and the same 'flat-catcher' is frequently sold at auction to the unwary till he becomes so well known as to excite a laugh among the habitués who attend the various metropolitan sale-yards.

Of causes connected with the feet producing lameness there are many ; among the commonest are pricked, stabbed, and wounded laminæ in shoeing. Many horses go lame from bad shoeing, though these are only a small proportion of the cases laid at the farrier's door. Farriers are not less intelligent or careful than other craftsmen, but unreasonable things are expected of them. While every novice with his first horse thinks himself capable of giving the smith instruction, old hands expect him to make cripples go sound, no matter what their defects. When it is actually proved that a horse has been pricked in shoeing, it does not necessarily follow that the farrier is to blame: a horse will plunge at the moment of driving the nail, or the horse standing next him be frightened by the 'sparks that fly like chaff from a threshing floor,' and sees more danger than poetry proceeding. Or a nail will sometimes 'sliver' or split at the end ; especially does this apply to handmade nails, which are rapidly disappearing with the starvelings who make them. Again, it often happens that a horse may 
be lamed in shoeing by the nail going so near the sensitive part, that it presses upon the quick and causes lameness after a few hours or days. There is no absolute rule to be laid down as to how soon a prick will manifest itsclf; it may show before the horse gets back to his stable, or fester after more than a week has elapsed. If he is observed to go 'feelingly' and the shoe is removed at once, he may be all right again immediately,

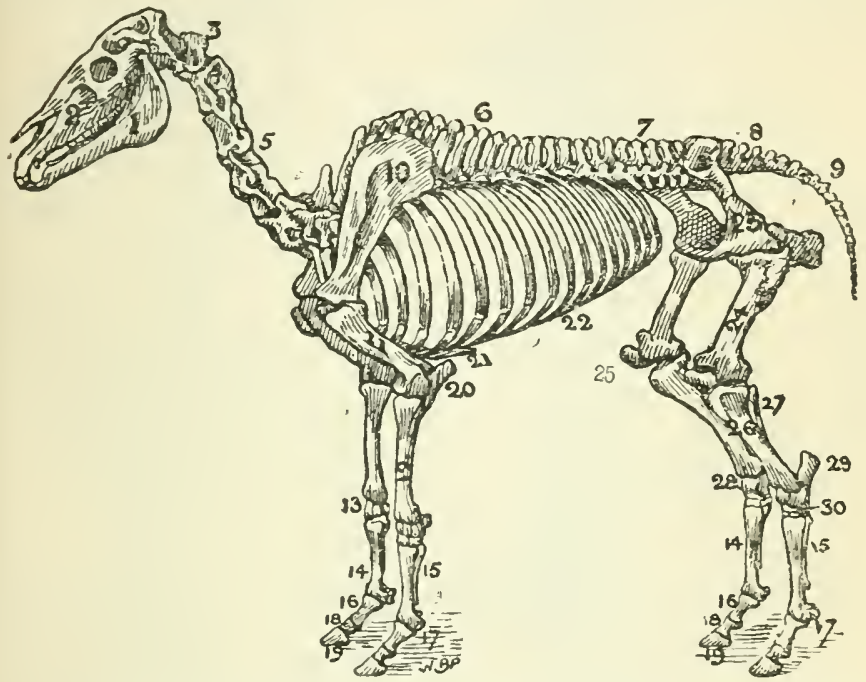

1, Lower jaw ; 2, superior maxillary ; 3 , atlas, first vertebra of the neck ; 4 , dentata ; 5 , remaining vertebræ; 6 , superior spincus processes; 7 , dorsal and lumbar vertebræ; 8 , sacrum ; 9 , coccygeal, or tailbones; 10 , scapula, or blade bone; $1 x$, humerus; 12 , radius; $x_{3}$, carpel, or knee bones; $x_{4}$, cannon, or shank bones; $x_{5}$, splint, or splent bones: 16 , long pastern, or os suffraginis; 17 , sesamoid bones; 18 , os corona, coronet bone, or short pastern; I9, os pedis, pedal bone, or coffin bone (navicular bone behind); 20 , elbow, or ulna; 21 , sternum, or breast bone; 22 , ribs; 23 , ossa innominata (including pelvis); 24 , femur; 25 , patella, or stifle joint ; 26 , tibia; 27 , fibula ; 28 , astragalus; 29 , os calcis: 30 , small bones of hock.

or he may not. Many horses get a 'stab,' as farriers call it, and receive no other treatment than the pouring into the wound of a little nitric acid. If this had not proved successful, or at least not harmful, in many thousands of cases, we should have said it was a barbarous custom; but as nothing succeeds like 
success, we must pass it by ; unless indeed we stop to note the fact that modern knowledge of microbes explains how entirely satisfactory a remedy is, that will at once and effectually
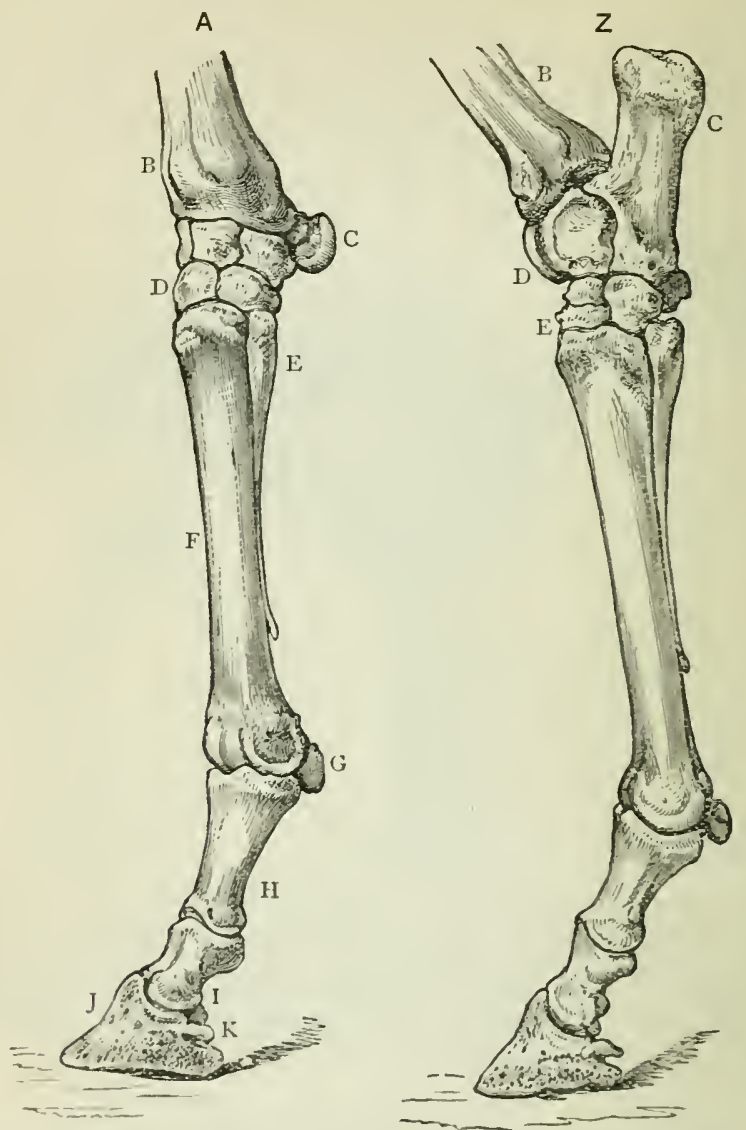

A FCRE LEG.-B, lower third of radius; C, trapezium; D, small bones of knee arranged in layers: $H$, splint, or splent bone; $F$, cannon, or shank bone; $G$, sesamoid bones; $H$, long pastern, or os sufraginis; 1, short pastern, or coronal bone; J, os pedis, pedal, or coffin bone; $K$, navicular, or shuttle bone.

Z Ilivin Li:G. 13, lower third of tibia: C, os caleis; D, astragalus; E, small bones of hock. 'The bones below the hock are designated by the same names as in the front limb. 
exclude those pyogenic organisms which give rise to suppuration. If a horse is lame, and the cause is not actually known, it is best to consult a veterinary surgeon. The diagnosis of lameness is one of the most difficult branches of veterinary practice, and the counter prescriber who would do justice to his client will not attempt it. If a prick is known to be the cause of lameness, no time should be lost in removing the shoe, and enlarging the nail-hole with a searcher, the practitioner not resting satisfied till the sensitive parts are discovered.

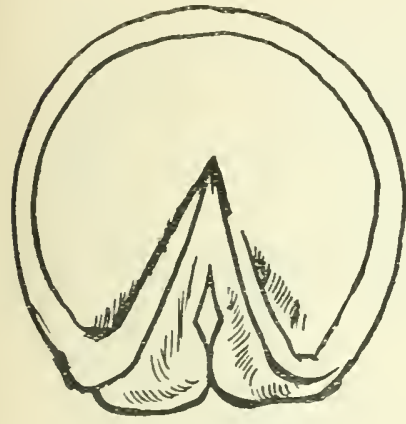

A Good Open Foot.

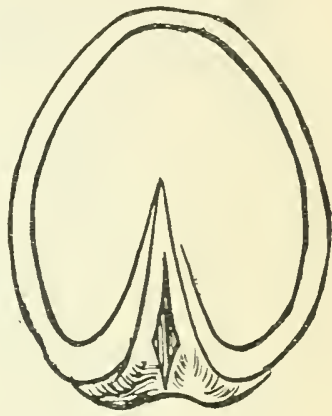

A Contracted Foot.

The degree of lameness from pricks seems to be out of all proportion to the extent of injury, and a horse may be unable to bear the least weight upon the foot, though only the tiniest drop of matter may be confined in it. When once given exit, he is greatly relieved. A matter like green sap is more often discovered than pus; it is decomposed horny matter of characteristic colour and offensive $\left(\mathrm{H}_{2} \mathrm{~S}\right)$ smell.

If pricks are not very quickly relieved by enlarging the orifice below, the matter makes its way upwards in the line of least resistance, coming out between hair and hoof, when it is a much more serious affair. If one is not called upon to treat it until this stage, a drain should be attempted below all the same, as sinuses are always feared, which, when once established, constitute quittor. 
The treatment of pricks consists in first of all having the nail-hole thoroughly pared out to give exit to any matter; then placing the foot in hot water for about an hour, after which a poultice consisting of equal parts of bran and sem. lini cont. should be applied. A fresh poultice should be put on every day for two or three days until there is no discharge, after which the following ointment on a pledget of tow should

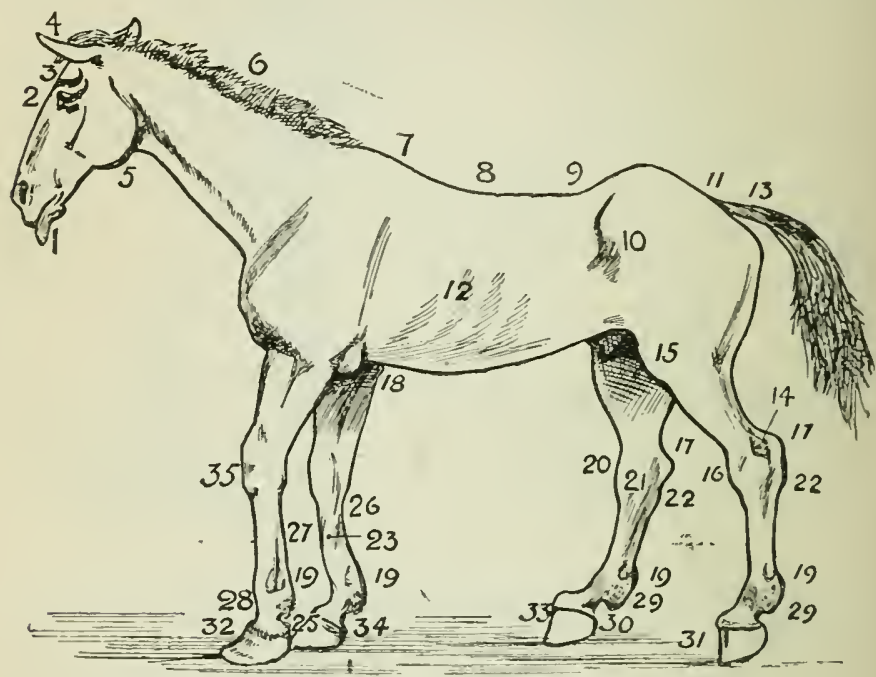

1, Pendulous lips; 2, cataract and amaurosis; 3 , hollow orbits; 4, pigs ${ }^{\circ}$ ears; 5 , enlarged glands ; 6 , itchy mane ; 7 , fistulous wither ; 8 , saddle-galls ; 9 , hollow-backed : 10, knocked-down hip; II, crupper galled; 12 , slab-sided; 13 , goose-rumped; 14 , thoro. pin: 15 , enlarged stifle ; 16 , bog spavin ; 17 , capped hock; 18 , capped elbow; 19 , wind galls; 20 , enlarged ligaments ; 2 I, spavin : 22 , curb; 23 , sprained tendon ; 25 , greasy heel ; 26 , contracted tendons; 27 , sprained back tendons ; 28 , shotover fetlock; 29 , enlarged sesamoids; 30 , cracked heels; 3 r, sand-crack; 32 , pumiced foot ; 33 , crown scab; 34 , quittor ; 35 , enlarged knee.

be introduced into the wound and kept there by means of strips of wood or hoop-iron wedged under the shoe.

Cerre Flav. . . . . ว j.j.

Adipis . . . . . . 3 ss.

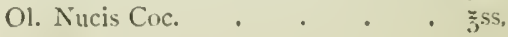

Plicis Arch, . . . . . ॠiv,

Ft. unguentunu, 
A doge of physic should also be recommended during the time the foot is being poulticed.

Cracked Heels are a frequent source of trouble to the horse-owner, and are generally caused by bad management. Some horses are constitutionally disposed to this troublesome complaint. It more often occurs in white legs than others, and is not peculiar to any particular brced or class of horse. Working in snow or crossing streams of cold water will account for a few of the cases met with, but the majority are caused by washing the heels after work, and allowing the moisture to evaporate and produce a chill to the sebaceous glands of the heel, which become thereby infiamed, and pour out an irritating material, which, instead of performing its usual function of keeping supple the skin, produces cracks, which, if neglected, go on to ulcers. Horses subject to cracked heels should not have the legs washed on coming in from work, but be brushed clean after the legs are dry. Even the carefully coddled hunter does not always escape, though the heels be dried and bandaged.

In a mild case of cracked heels mild remedies are best, such as either of the following :-

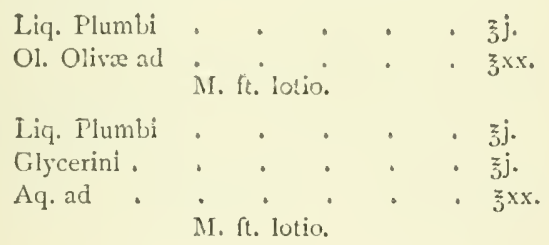

If the heel has already become encrusted with dry matter, the former will soften it, and enable the animal to work with less likelihood of aggravating the affection; but the glycerine preparation will be found to allay inflammation better even if the heel be very red and tender to the touch, but not actually discharging matter.

In very bad cases, rest, poultices, and fever medicines become necessary. The poultices should contain a little liq. 
cárb. deterg., or Jeyes's fluid, or acid. carbolic. I, to glycerine 20 parts. Equal parts of sem. lini cont. and bran are to be preferred, but the practitioners in some out-of-the-way places will have to make shift with turnips, or anything that comes handy.

Cracked heels also occur in dry summer weather, and are not traceable to any mismanagement, but rather to the irritating effects of the dust settling in the heel. When caused in this way greasy preparations are perhaps the best, and among these ung. zinci carb. has a good reputation where the ung. zinci ox. is too irritating. Professor Simonds used to recommend pulv. zinci ox. mixed with flour, and mentioned an obstinate case which was finally cured by flour alone. In the summer, when flies are troublesome, a little tar or phenol should be added to the ointments. When ulcerated cracks are developed, they need treating with powerful astringents, which should be carefully applied to the ulcers only. For this purpose we have found the following better than anything else :-

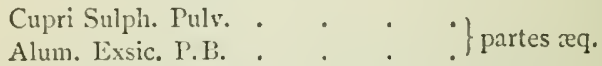

This may be dispensed as a lotion if preferred, dissolving a drachm of each in aqua 5 vi.

The milder astringents are to be preferred in the treatment of cracked heels, as the union is the more lasting if not hurried. Only in chronic gaping sores with indurated edges should we resort to the last formula. Cracked heels is a condition always liable to recur, and should be looked upon with suspicion when making a purchase.

Corns.-A corn is an injury to the horn of the foot, at the same time involving the tissues bencath, causing rupture of the minute blood-ressels and the escape of blood, which, by permeating the horn in the neighbourhood, gires it a dark colour when cut down upon. Corns are usually situated on the inside of the foot, between the bars and the wall of the heel. Many horses have such corms, and never suffer lame- 
ness while properly looked after. If, however, the farrier fails to keep a corn pared, or puts on a shoe having a bearing upon the part, lameness soon results, and festering too often follows. The shoeing-smith, or farrier, as he is more properly called, usually gets all the credit of producing corns in horses; but in this and many other cases he gets more than his share of the blame. Corns much more often arise from the owner's meanness or the groom's neglect than from the farrier's method of shocing. While the horse's foot continues to grow the shoe does not, and the shoe that once had a bearing on the crust only, finally presses upon the seat of the corn, and produces the mischief we have now under consideration. If the horse-owner insists upon wearing out shoes to the thinness of a sixpence, no matter how long they may have been on, he has only himself to blame if corns

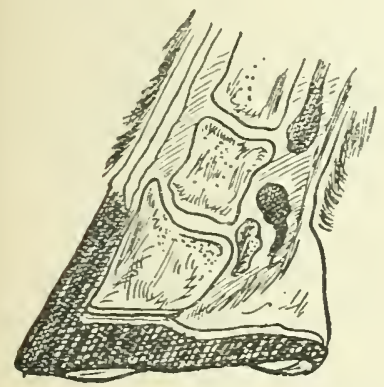

Section of Pastern and Foot.

Showing portions of Lons Pastern, Short Pastern, Pedal Pone, Navicular, \&c., \&c.

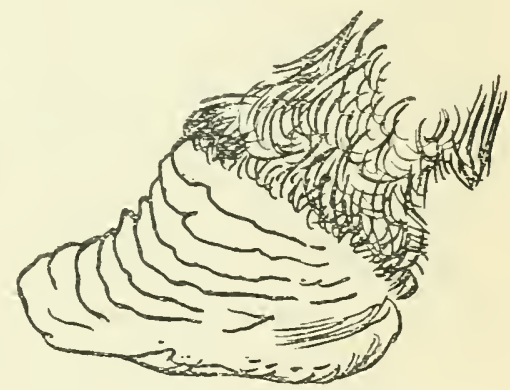

A Bad Case of Laminitis or Fever in the Foot.

result. Some horses with low; flat feet, like those of the Netherlands, have a predisposition to corns, while the upright feet of the Arab type are not so susceptible.

When a corn festers and causes lameness, the knife must be used freely to lay it bare, and ung. resinæ, or other drawing or 'digestive' material, applied, and the whole foot should be enveloped in a poultice. If this is done promptly 
relief is soon obtained, and the parts may be hardened off with liq. antim. chlor. or tar on tow, and the shoe replaced so as to have no bearing upon the tender parts. If this is not done in good time the matter will break out at the coronet (see Quittor). Bar-shoes and other contrivances help to reduce the bad results of corn, but it is a serious objection to a hoŕse, and always liable to be a cause of lameness.

Ring-Bones.-This is a term given to an abnormal growth or deposit of bone upon the pasterns or lower bones of the legs. There are two pastern bones, called respectively the long and short, or Os suffraginis and Os corona.

All breeds of horses are liable to ring bones, but heavy draught-horses are especially liable, as their bones are short as compared with the blood horse, and more upright, and concussion is more violent as a consequence. The arrangement or anatomical construction of the horse's foot and leg is such as to minimise the chances of concussion and subsequent inflammation; but it must be remembered we are almost always dealing with animals in an arificial state when we are asked to prescribe for lameness or deformity. Ring-bone may occur on the front or hind pasterns; it may be on the upper bone, when it is called 'high ring-bone,' or on the lower, just above the hoof, when it is denominated 'low ring-bone,' and must be distinguished from side-bone, of which we shall treat hereafter. It is often hereditary, and found upon the same horse as splint, spavin, and other exostoscs, as growths of bone are technically termed. It should be remembered that the growth of a ring-bone is from the outside. Bones do not grow from within outwards, but from the periosteum, or covering membrane, and when by concussion this membrane becomes inflamed its function of secreting bone is excited, and calcareous phosphatic matter is produced in excess. Unless ring-bones happen to bc an hereditary production, the animal having a bony diathesis, or predisposition to throw out deposits of bone, they are caused by a greater strain than the existing bones can endure, and the production of more bone is within certain 
limits a physiological process destined to prevent the recurrence of the strain by providing against a like contingency. The process and the products of inflammation are now regarded in a totally different manner from that adopted formerly. Inflammation of any structure is in reality an effort of nature to accommodate the parts to altered circumstances. This may best be illustrated by reference to the human hand. The soft palm will blister with an hour's rowing; the blister is the result of inflammation. The sequel is a corn, and the rower's altered condition will soon enable him to use the oars without blisterirg. If he leave off rowing, and the corns are no longer required, nature will absorb them or cast them off. If this is torne in mind in the treatment of horses, good results will follow. All ring-bones do not require the same treatment. We will suppose a client has a young cart-horse which has fallen lame with incipient ring-bone. This is the time to use a sedative or evaporating lotion, in order to modify the extent of the inflammation, and not produce a lot of bone, which will be an eyesore and a detriment to the horse. In such a case an excellent lotion may be made as follows :-

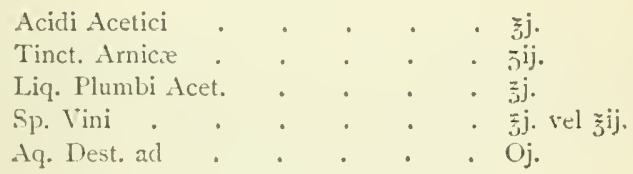

I. ft. lotio.

To be applied on a wet swab or bandage, renewing it fre quently. In a young horse this will probably so reduce the irritation in the course of a few days that he will go sound again; but the cause must be removed, and the colt turned out to grass till his bones become more consolidated and fit to bear the concussion and strain of starting heavy loads on macadamised roads. If the owner cannot or will not be persuaded to turn the colt out, or, as often happens, has not the capital to invest in another, then blistering must be resorted to. Blistering will probably not remove the ring-bone, but it will absorb 
some of the deposited material, and, by thickening the skin and subjacent structures, give increased support to the parts, as does the corn upon the rower's hand. It often happens that, with a steady driver or considerate carter, a ring-bone will entirely disappear, the horse never being again subjected to so severe a strain as that which caused it, and its absorption being undertaken by nature because found to be unnecessary.

This does not apply to hereditary ring-bones ; their absorption is seldom accomplished with or without treatment. A great many cart-horses have ring-bones without experiencing any particular inconvenience, and they are best let alone unless there is lameness or a palpable increase in their size, when blistering should be resorted to. There are blisters and blisters, and before deciding what to use the question should be asked at what age and under what circumstances has the subject developed ring-bone. If it be a recent case, a cantharides blister will do well cnough. If of long standing, then

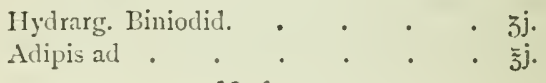

M. ft. ung.

should be employed. The biniodide is generally preferred for all bony enlargements.

Three or four ounces will be required for a cart-horse's leg even when clipped tolerably close. All veterinary preparations should have an allowance for waste; if it be an ointment some will run down and fall off, and if it be a drench some will be spilt. 'Half in and half out, like a farrier's drench,' is quite a stable proverb. A practical prescriber will not fail to tell his client to tie the horse's head up for at least two nights and days, lest the patient should gnaw the parts, damage the shin irreparably, and blister his own nose, a most unsightly accident. $\Lambda$ basket suspended from the ceiling can be used for the food if the horse shows a disposition to strike the manger with a front leg that has been blistered. Whenever a blister is prescribed for any of the lower parts of the legs the owner should be cautioned to remove the straw, as much unnecessary pain 
and sleeplessness are produced by the ends tickling and irritating the blistered surface.

On the third day after blistering, a simple ointment should be used to soften the skin and prevent cracks and ulceration; this is very grateful to the patient and will usually prevent him from gnawing it when his liberty is restored to him and the opportunity.given to lie down. Blisters indifferently applied cause pain without benefiting the animal, and humanity demands that we shall take every care to apply them properly, not in a perfunctory manner, requiring repetition, or, as with acid. sulph., causing sloughing of the skin and permanent blemish. Careful veterinary surgeons, after seeing that the limb to be blistered has been properly clipped, begin the operation by putting a little ung. simplex into the heel, as that is the part most likely to crack and cause lasting trouble.

The following is an effectual blister, and in much request by reterinary practitioners :-

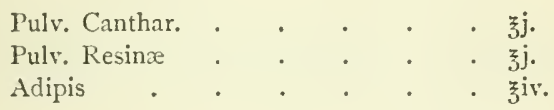

The lard and resin to be melted together, the cantharides added and stirred till cold.

Large surfaces should not be blistered with cantharides, and not more than two legs at one time, as absorption and kidney disease have been known to occur.

Preparations of hyd. bichlor. and blisters containing ol. terebinth. or tereb. venet. should be avoided : they are extremely painful, liable to produce sloughing, and not lasting in their effects, while the effect of a biniodide blister is often observable for months after application.

If a horse is gross and uisposed to ha swelled legs, he should be kept on bran mashes for twenty-four hours, and get a physic ball of from four to six drachms, according to his size.

Side-Bones are a frequent cause of lameness, especially in the formative period, but the majority of heavy 
draught horses have them to a greater or less extent, and may work sound for years despite them. Farm horses are not so liable as those subjected to the concussion of paved roads.

Side-bone is a conversion of the lateral cartilages found on the wings of the coffin-bone into bony material by the deposi-

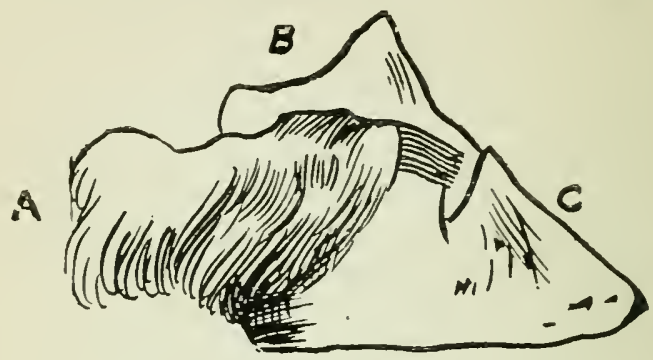

A. Lateral Cartilage, in which side-bone forms.
B. Short Pastern Bone.
C. Pedal or Coffin-bone.

tion of lime salts. They usually only affect the fore feet. The treatment is much the same as for ring-bones.

Spavin is a frequent cause of lameness in the hind limbs, though there are bony deposits, known as knee spavins, in front legs. A sparin, when not otherwise defined, may be taken to mean an alteration of the hock on its inner surface. In old works three kinds of spavin were described under the title bone, bog, and blood spavin ; but the latter is now seldom heard of, being in reality but a varicose condition of the large vein (saphena) passing over the seat of spavin. Bone spavin consists of a deposit of bony matter on the inner surface of the hock at or about the junction of the shank-bone (metatarsal), the splint-bone, and the lower row of small bones of the hock. It arises from concussion and subsequent inflammation of the periosteum, and results in more or less enlargement at what is commonly called the 'spavin place.' 'The majority of cases yield to treatment or become 'callous' without any treatment, and beyond the fact that they circumscribe the sphere of move- 
ment of the joint, may not be any great source of trouble, though always constituting unsoundness, since an old spavin may at any time cause trouble. (Unsoundness is defined as 'anything which does now; or may hereafter, interfere with the usefulness of the animal.')

The treatment commonly adopted is that of blistering and rest, with, if possible, a run at grass or three months in a strawyard. In blistering for the cure of bony growths we should always advise hyd. biniod. of a strength varying from i to 7 to I to 2 of vaseline, or lard, or lanoline. Some veterinarians prefer goose-grease as more penetrating, and having in itself a mollifying influence upon stiff joints.

Bog spavin is a soft enlargement in the front of the seat of bone spavin. It may be only an enlargement of a bursa-one of those lubricating sacs filled with a glycerine-like fluid, and placed upon all the points most liable to friction-or it may be associated with thoropin or a dropsical condition of the whole joint. Continued pressure, by bandages and other special apparatus designed for the purpose, will often reduce recent cases, but a blister repeated in a month with rest is a more effectual remedy; since the thickening and contraction of the skin that follow act as a permanent bandage. 'Charges' are of especial value for the treatment of these bursal enlargements (see p. 38). Firing produces a still firmer bandage, as the elasticity of the skin is removed by the new and inelastic material which nature produces to fill up the gaps made by the iron; thus it is that firing sometimes succeeds when all other remedies have failed.

False Quarter is a fault in the hoof produced by some injury to the coronet, or coronary band, from which the outer wall of the hoof is secreted. It frequently follows upon treads, quittor, \&c., and there is not much to be done for it.

Tread, or Over-reach, is a bruised or wounded coronet caused by over-reaching or treading one foot upon the other in turning or backing. It is generally among heavy draught horses 
that it occurs. Rest, poulticing, and emollient applications, as ung. cetacei $z_{j}$., acid. carbolic. mx., generally effect a cure, unless the injury is too deep, when sloughing ensues, and sometimes quittor and false quarter. If a slough soon comes

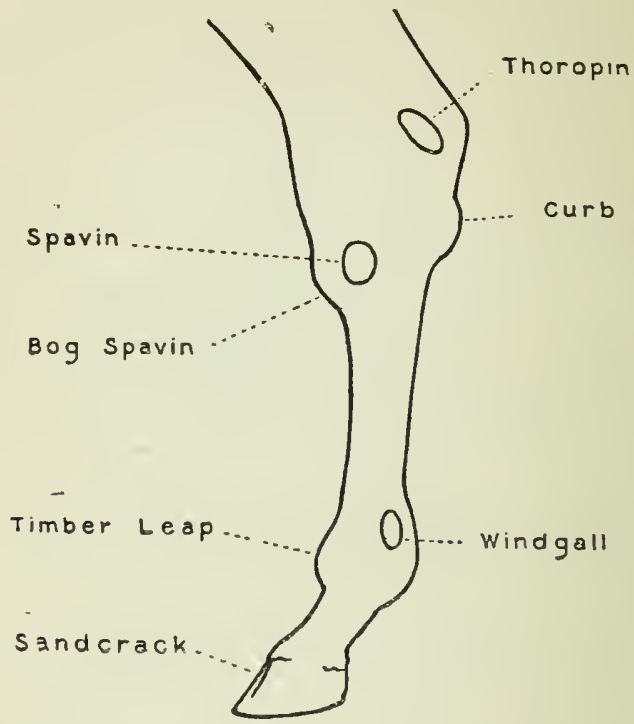

away with poulticing and emollients, a healthy wound is left, which may be very well treated with tinct. benzoin. co. or ung. picis.

Sand-crack is a division in the wall of the foot. In the forefoot it generally appears on the inside, and at the toe or in front of the hind feet. It is a serious defect, and should be looked for in purchasing a horse, the crack being sometimes neatly filled up with shoemaker's wax, and blacked orer in such a manner as to escape the eye of the unwary.

'Treatment consists in taking the bearing off the shoe, approximating the cracks by screws and other mechanical contrivances, and by stimulating the coronary band to form new 
material by blistering it repeatedly with some mild blister such as hyd. biniod. I to I2. Firing a $V$ in the hoof so as to lcare the top of the crack in the centre of the $V$ is a very good plan; the concussion of the foot is thereby diverted from the injured part of the coronet, and gives it the nearest approach to rest that can be obtained.

Canker is a disgusting fungous growth occupying a greater or less surface of the sole and frog. It is seldom curable, though it may be kept in check with nitric acid and other escharotic and caustic agents combined with pressure. It is better left to the regular practitioner, aided by the smith, and consummated by the knacker. Stripping the sole is sometimes resorted to, but is a very severe operation and of doubtful advantage. There are not, however, wanting authentic instances of recovery due to such treatment.

Seedy Toe is an imperfect or perverted secretion of horn, a cheesy kind of material being produced and causing separation between the horny laminæe and the crust. It is not confined to the toe, but derives its name from being most often found there.

Treatment consists in removing all the defective horn and stimulating the growth of new material by blistering the coronet and stuffing the foot with tar ointment, $\mathcal{S}$. It is an occasional cause of lameness, and constitutes unsoundness. It may easily be detected by tapping the unsound foot with a hammer, and comparing the sound with that of a healthy one.

Navicular Disease may be said to cause more lameness of the chronic form than all others put together. It begins as concussion, and ends in ulceration of the navicular bone. It is hereditary, and often comes on very early in horses working on the stones. No class of horse is exempt from it, but light horses with good action are the most, and heavy horses the least, frequent subjects. 
Pointing one foot in front of the other alternately and ad vancing the opposite hind leg is a common symptom. Many horses are able to work though suffering from this disease, as the lameness goes off with exercise and only returns after standing some little time. It always grows worse, however, and is a very serious example of unsoundness if showing even to a slight extent. Blistering and a run at grass appear to cure it, but if the history of the patient can be traced it will generally be found that the operation of dividing the sentient nerves (neurotomy or neurectomy) has to be resorted to. Some horses go sound (but without any feeling in the feet) for several years, while others have to be killed on account of the hoof sloughing off, or of the tendons breaking away and bringing the heel down, and the toe up.

Thoropin is a soft enlargement between the bones of the hock and the point (os calcis, see p. I2I), and derives its name from the fact that pressure on one side will make it bulge on the other as though a bolt or pin were run through it. The same cause gives rise to it as to bog spavin, and the treatment should be the same.

Blister charges cnveloping the whole joint save at the point of the os calcis are recommended.

An animal may have both bog spavin and thoropin without lameness, but both defects are apt to increase unless timely treatment is adopted.

Curb is another source of lameness, and is a sprained ligament at the back of the hock-joint. It is of frequent occurrence in young horses put to work before their structures are capable of bearing the necessary strain entailed in jumping or other violent efforts. Many Irish horses are fired over the seat of a curb as a prccaution against it, and must not be rejected as the subjects of curb for the simple reason that firing-marks are to be observed.

Fomentation, cooling lotions (see Sprains, \&.c.) may be used with advantage, but in screre cascs a b!ister, or e:en firing, may 
be necessary, and some slight enlargement will still remain, though the ligament nay have become as strong as ever. It is an unsoundness liable to recur.

Sprain.-Sprain, by which is meant a stretching or even partial rupture of a ligament or tendon, raries in degree according to its severity and situation. Sprain of a joint ligament may induce organic disease in the joint itself; sprain of a tendon may be followed by permanent thickening and defective action. No matter how slight a sprain may appear, it should be carefully treated. The most common error in the treatment of sprain is confusing the acute with the chronic stage, applying cold to a chronic injury and stimulants to a recent one. The objects of treatment are in the first stages to keep down inflammation and prevent exudation and swelling ; in the second to stimulate absorption, promote repair, and guard against complications. In the first stage either hot or cold water may be applied; but whichever we use must be kept up continuously, so as not to produce a reaction. Cold water is preferable if the injury be quite recent-not if much swelling and congestion exist. With the water some medicament is expedient. If cold is used, either tincture of arnica or spirits of wine may be added (the spirit is probably the useful ingredient in the tincture), or the following lotion, which is excellent :-

Potass. Nit. : : $\quad$ :
Ammon. Chlor.
Sodii Chlorid. :
Aq. $\quad$ :

If warm water is used, some anodyne may be added, as belladonna or opium.

The treatment must be continued until all violent symptoms have passed off-till the intense pain and heat are gone ; then a dry bandage and occasional mild hand-rubbing may be adopted for a day or two, after which some stimulant is needed to promote absorption and repair. In ordinary cases a blister is not allowable; it is too active and its effect is of ton short 
duration. It is better to adopt some milder treatment and continue it longer. There are many good applications. Liniments of ammonia and iodine, and tincture of cantharides are all good. They should be applied daily in small quantities and with moderate friction.

A very good stimulating liniment may be made as follows :-

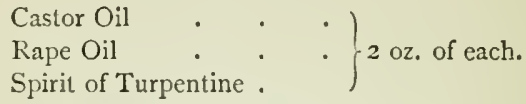

Shake, and then add-

$$
\begin{array}{ll}
\text { Strong Solution of Ammonia . . . } 3 \mathrm{oz} \text { of each. } &
\end{array}
$$

This preparation has the merit, too, of being elegant, and can be diluted with water in proportion to its own bulk, without losing in appearance. Should this treatment not effect a cure, the part must be blistered once or twice, and, as a last resort, firing and a run at grass may be necessary. Rest in all cases is absolutely essential, and that too for a considerable time. Cases seemingly incurable will frequently come up sound after a summer's run. While confined to the stable an animal should not be allowed much corn; bran should be used with grass or hay, and the bowels should be kept open with physic if necessary.

Capt. Hayes, F.R.C.V.S., is a great advocate of wrapping a recent sprain with cotton wool, and a bandage outside to afford even and gentle pressure.

Thrush.-Every horseman knows this disease of the horse's foot by sight, many know how to cure it, but few know the cause, and thus its prevention is neglected. Doubtless dirt is the exciting cause, but dirt alone seldom produces a bad 'thrush.' Heavy cart horses are of all most subjected to dirty roads, and their feet are most neglected in the stable. Nevertheless, with few exceptions, they are free from this evil. The horses of gentlemen and dealers are not as a rule subjected to much dirt; they have the greatest attention in the stable, and 
yet they are of all classes most troubled with thrushes. The explanation of the difference is probably that cart horses are neglected by the horse-shoer, while the feet of the lighter horses are trimmed and pared so as to please the eye. In a state of nature the horse's frog comes down on the ground at each step. When shod, and especially if much pared, it seldom gets the slightest pressure. Now this pressure appears to be necessary to the healthy growth of the frog. Just as a man's hands become hard and strong by work, or weak and tender by idleness, so the horse's frog, by being never brought into use, gets thinner and weaker. Want of pressure then, we say, is the predisposing cause. It will generally be found that horses standing in a stall have the hind feet affected more frequently than the fore; the reason of course is that the hind are most exposed to the excreta of the animal.

Thrush, unless it be constitutional and in the nature of an issue, is not difficult to cure, and is just one of those complaints for which the prescribing chemist is often consulted.

It is a fœtid discharge from the frog, and its disagreeable odour is characteristic of decomposed horn. The frog presents a rugged appearance, with holes in it containing decomposed matter, but the bulk of the discharge is from the cleft.

The treatment is very simple. First thoroughly cleanse the foot with warm water and remove all loose portions of horn ; then apply some mild astringent, and stimulant. The discharge should not be dried up suddenly by any strong dressing, as a swollen leg is a not uncommon sequel. The following is good :-

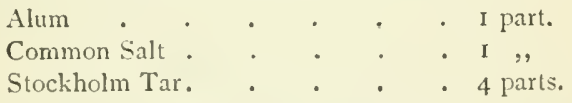

Some alterative balls should be prescribed in cases where the disease is chronic and constitutional. They should contain a drachm of aloes, and otherwise their composition will be that of the common diuretic ball.

Should the case prove obstinate, substitute for the salt sulphate of zinc. To avoid thrush in the stable, the master 
should give orders that the frogs are not to be pared at the forge, but that merely the loose portions of horn are to be re. moved.

A bar shoe is often an aid to restore a healthy secretion by exercising pressure.

Many chemists are possessed of 'a good remedy for the thrush.' More often than not these remedies are too good; they dry it up and shrink the frog away if applied properly, or fail to penetrate the cleft and do not accomplish their object. In curing thrush in horses or foot-rot in sheep a frequent difficulty consists in getting the diseased parts carefully trimmed away, and the cleft cleared out. The latter should be done with a flat piece of stick; if this be carefully seen to in a thrushy foot, very strong caustic remedies are not necessary. A very good thrush-dressing is the following :-

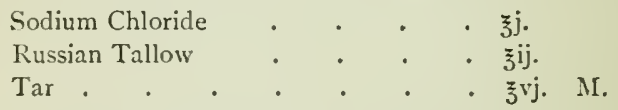

Tinis inelegant preparation removes the disease, and prevents the drying up and shrinking of the frog. It is quite capable of being improved by our pharmaceutical readers in such a way as to look and 'nose' better without losing any of its merits as a curative agent. It is equally good for foot-rot in sheep. Where the use of a powder is desired the following may be recommended :-

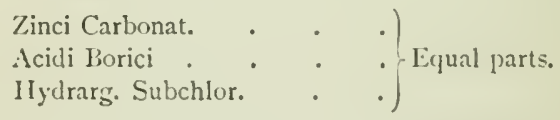

Calomel is almost a specific for thrush. If used alone it may be coloured with a little bol. armen.

Wind-Galls, so called, are occasionally; but not often, a cause of lameness; they usually indicate hard work or premature use of a horse, and reduce the value, however little they may. interfere with the usefulness of the animal. They can scarcely be called an unsoundness, unless hard and inflamed or giving evidence of pain, which is rare. They were formerly supposed 
to contain air, hence the name, but they are really enlarged or ciropsical burse (see Bog-spavin). The pressure of a bandage will reduce them temporarily, and if constantly applied prevent them getting worse, whilc blistering and rest will for a time quite remove them. A bandage dipped in glue is a capital remedy for their reduction when a few wecks' rest can be procured. They should on no account be pricked or opened.

Rheumatism in Horses. - There are many obscure cases of lameness in the horse which the veterinary attendant is obliged to assign to rheumatism ; and some inflamed joints, where the cause cannot be traced, may possibly arise from that condition of the blood. We are, however, inclined to regard rheumatism in horses as very rare, and the name is but a cloak for ignorance in nine cases out of ten. If horses were subject to rheumatism as a result of wet and exposure, we should be called upon to treat them most frequently when lying out through the winter exposed to the elements. But this is not. often the case. It is more often the stabled steed which is affected, and many so-called cases of rheumatism of the shoulder turn out to be navicular disease of the foot. Its shifty character from one limb to another is often quoted as a characteristic of the disease, but the same thing applies to laminitis and other inflammatory complaints, and it is not yet at all satisfactorily proved that true rheumatism is a disease of the horse. For the pains known by that name pot. nit. and pot. bicarb. may be given in doses of

$$
\begin{aligned}
& \text { Potass. Nit. . . . . } 3 \text { ij. }
\end{aligned}
$$

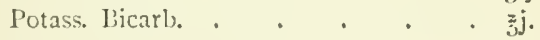

$$
\begin{aligned}
& \text { Or Salicin . . . . . oj. to jjse. }
\end{aligned}
$$

daily, and the application of belladonna, opium, and aconite.

A very good liniment is the following :-

$$
\begin{aligned}
& \text { Lin. Aconiti • • • • . } z_{3}^{3} \text {. } \\
& \text { Lin. Belladonnce . . . . } 3 \mathbf{i j} \text {. } \\
& \text { Tinct. Opii . . . . } \xi_{3} \text {. }
\end{aligned}
$$

M. ft. liniment. 
Blistering is also recommended in the case of swollen and inflamed joints supposed to be due to rheumatism. The scientific reason given by Dr. Davies of the London Hospital is that blisters act as eliminatives. Whether this theory be correct or not, experience for hundreds of years has proved that blisters give relief to swollen and painful joints from almost any cause save the presence of matter.

The same treatment applies to cattle, but the doses of salines may be increased by one-third.

\section{Laminitis, Founder, or Fever in the Feet, is a} disease of the horse's foot, in which the sensitive layer immediately within the hoof is congested, or even inflamed. It is commonly caused by overwork, such as a long journey on a hard road, or hard work in horses out of condition.

Symptoms. - First, frequent shiftings of the feet, and signs of pain, as quickened breathing and pulse. Next, fear of raising one foot lest extra weight be thrown on the other, with swaying of the body backwards and forwards without the feet being moved. If force is used the animal moves as though his back was injured, and puts the heel most markedly on the ground. When the forefeet alone are affected, as is usually the case, the hind feet are drawn forward under the belly, and the animal leans backwards so as to lighten the weight on the front limbs. The affected feet are hotter than usual, and throbbing is felt above the coronet.

Treatment.-Never bleed. (rive an aperient (ol. lini Oj.), to which may be added tr. opii $\tilde{z} \mathrm{j}$. or several doses of sodæ salicylas $5 \mathrm{ij}$. ; remove the animal to a smooth, hard-bottomed loose-box, with a light covering of clean straw. The shoes must be removed. It has been found possible to induce horses to stand during this operation by injecting cocaine into the coronary band when they could not otherwise be induced to do so. Give a good broad bearing-surface with the ras]', but leave the sole untouched. If the foot is level when the shoes are removed, lower the toe and heels a little, to produce a sort of rocking surface. This relieves the pressure on the front of 
the foot, which is the part most affected. Use warm fomentations and bran poultices till the acute pain subsides, but no longer. Then use hand-rubbing to the limbs, and give gentle walking exercise. Have 'rocker' shoes put on-that is, plain shoes thin at the heel and at the toe. Simple cases generally recover in a week, or at most two. Some cases are very tedious, and result in 'pumiced foot' $-\mathrm{a}$ permanent incurable deformity ; careful shoeing will keep going even such animals.

Capped Hock is an unsightly enlargement on the point of the hock. It is at first soft and fluctuating, and at this stage treatment yields the best results. It is seldom a cause of lameness, but, taken in conjunction with other blemishes on the hind legs, it may mean vice; if not kicking in harness, it may indicate a restless animal that kicks the stall post or his companions instead of sleeping at night. It may be, and is generally, caused by insufficient bedding or a habit of scraping away the litter, and so bruising the hocks upon the floor of the stable. It is often associated with rubbed or sore places in the hollows outside the hocks, which are hollows only when the horse is standing or the rulss would not take place at that part.

It is usually only a bursal enlargement. Old practitioners were very fearful of operation, but there is really nothing to fear, and a small seton may be used to run it off if evaporating lotions and a deep bed of moss litter fail.

A liniment of equal parts lin. saponis and aqua dest., with massage, night and morning, has often proved successful in recent cases.

Repeated blisterings sometimes remove, but at other times only make the skin thicker and the hair coarse. Removal of the cause is the chief thing to be attended to, as many capped hocks sulsside without any treatment at all. If it is hard and callous and of long standing, it had better be sulmitted to as a permanent eyesore.

Splints are bony growths found upon the inside of the forelegs very frequently, but occurring also on the outside and 
less often on the hind limbs. They are deposits of bone upon bone, brought about by inflammation of the periosteum or bonesecreting membrane which covers the splint or splent bones in common with others. They are so common on the inside and between the knee and pastern as to excite little comment, and the majority of horses have them at some time during their
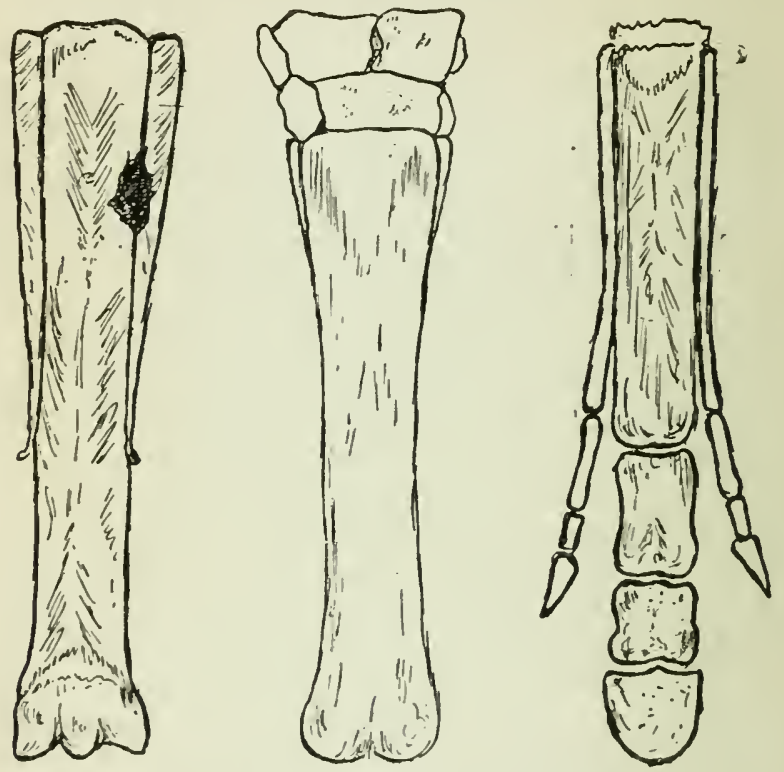

Showing Conditions of Leg-bones in Splints.

lives. It is during the formation of them, when the membrane is inflamed, that lameness results, and it usually passes away when, to use a common term, they become callous. Theoretically they constitute unsoundness, but the best veterinary surgeons decide rather upon their position on the limb, the age of the horse, and other circumstances, when passing such animals with a certificate of practical soundness. If a young 
horse of four or five years is being purchased from country work for trotting on the stones, the examiner would hesitate to pass a splint, but seasoned horses are well known to suffer but seldom, and therefore need not be rejected for splints, if situated well forward so as to be out of the way of the tendons and not likely to be struck by the foot of the opposite leg and not too high up, as near the knee is the worst of all situations.
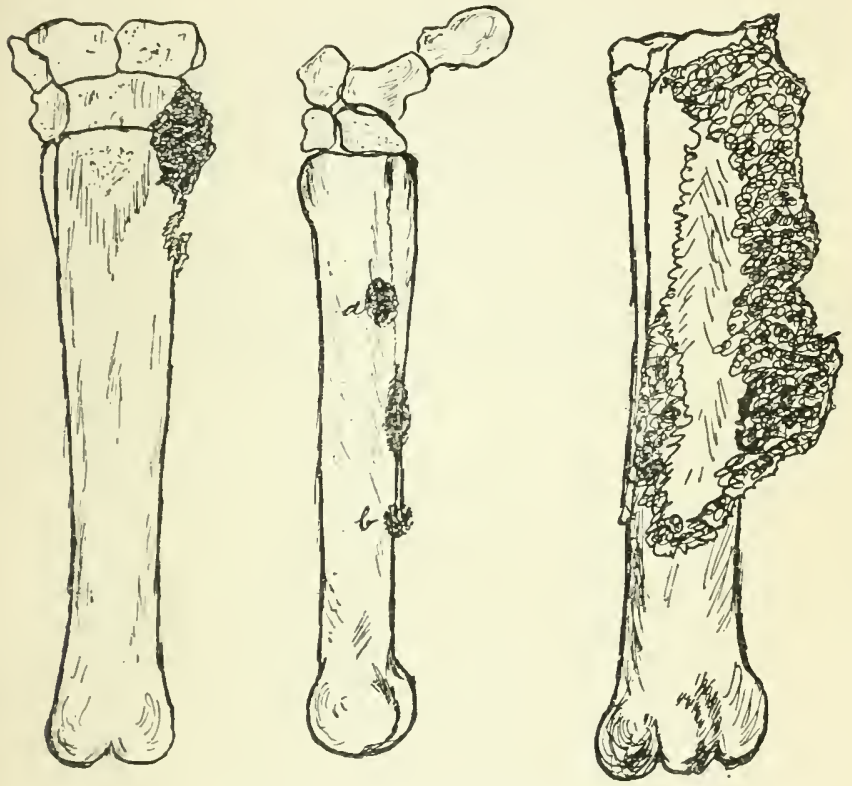

Showing Conditions of Leg-bones in Splints.

The lameness is not alway's easy to diagnose by the manner in which the horse goes, but it generally comes on when he has been out some time, while foot lamenesses frequently get better on the road-warm up, as it is called. If lameness is caused by a recent or newly formed splint, the animal should be taken off 
work and given a dose of physic, proportionate to his size, cold applications in the form of ammon. chlor. $\xi_{\mathrm{j}}$., aq. $\xi \mathrm{xx}$., on bandages for two or three days, when, if the inflammation has subsided, he may be given a week's rest and again put to work, but if he fails, or if the cooling method does not succeed, it will be better to blister him with hyd. biniod. one part, vaseline or lard eight parts. At least a month should be allowed after blistering and before putting a horse to work. We have known splints to be absorbed by painting with tinct. iodi twice a week and keeping a horse at work, but the lameness has not been great or such a method would not be advisable. Sometimes old splints are wakened up by sellers who tiy blisters to get rid of them. If they do not cause lameness they had better be let alone, on the principle of 'letting sleeping dogs lie.'

Sore shins is also a periosteal inflammation, and brings about a bony deposit; it is more often met with in racehorses than others. The treatment is the same as for splint. 


\section{DENTITION OF THE HORSE}

THE periods at which the teeth of animals are cut and the appearances they present are so regular that for the most part their ages can be told within a very little. The significance of the phrase in common use 'over seven,' as applied to people sufficiently sharp, is derived from the fact that when a horse is past seven years his age cannot be told with certainty, though good judges can form an approximate idea by the greater or less signs of wear and altered position of the teeth.

The adult horse should have forty teeth. At the birth of a foal the first and second grinders and molar teeth have already broken the gums, and at the end of a week, if not sooner, the first pair of temporary incisors make their appearance ; within six weeks the next or lateral pair come through, and the outer lateral or last pair come through between the sixth and ninth months. The molars are meanwhile making progress, but for telling a colt's age the 'nippers' or incisors are a sufficient ordinary guide. The number (six) being complete in the upper and lower jaw, no further changes beyond wear upon the surfaces take place until the permanent teeth begin to come. A yearling may, however, be distinguished from a two-year-old by a glance at the teeth; at one year they appear to be the right size for his mouth, or at least not too small; they are even and approximately the same size, though not actually, for in the mouth of the foal, as well as the adult horse, the central nippers are always larger than the rest. In a two-yearold the mouth has grown too big for the teeth, and they look small and worn on the crown, and spaces are beginning to form between the fangs. We are speaking, of course, of the 
common appearances, but there are instances of colts being so well developed in frame and the teeth so regular that they have been sold for five-year-olds with complete mouths.

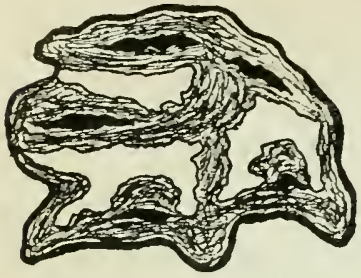

Transverse Section of Molar.

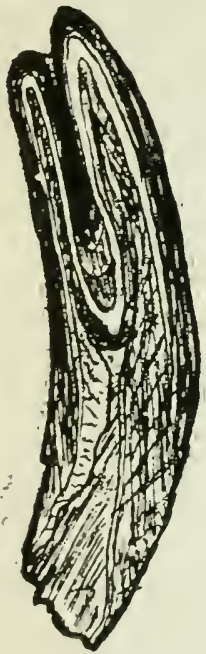

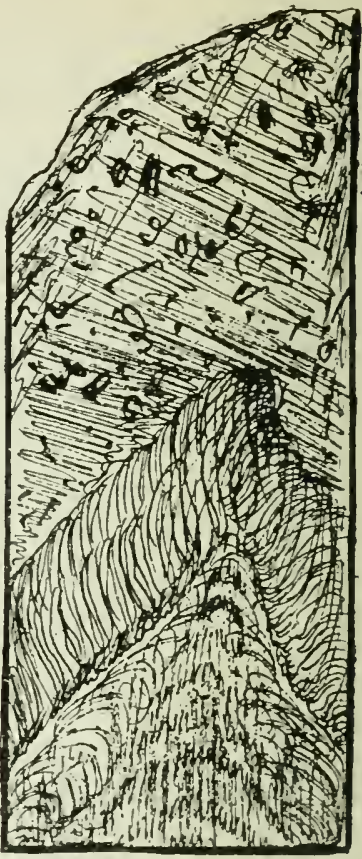

Longitudinal Section of Horse's Molar Tooth.

Longitudinal Section of Incisor.

The permanent nippers are larger, and intended to bear more wear. The first pair should displace the central tem. porary ones at from two and a half to three years. The absorption of the fang of the temporary tooth is usually advanced enough at two and a half years to cause it to fall out and the permanent tooth to break the gums. 'The greater size of these tecth enables onc to distinguish at once an animal 
in its third year. The next pair of incisors are cut a year later, at about three and a half years, and the last pair at about four and a half years, so that at five years the mouth should have all the teeth present though the outer ones will still be

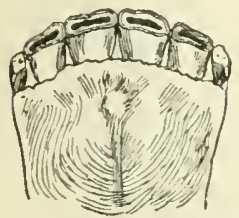

Four-year-old Mouth, showing four Permanent Incisors. The corner ones ready to be shed.

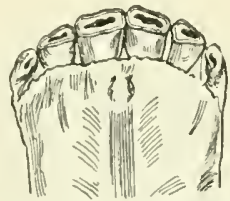

Front Teeth at Three Vears Old, showing two Central Permanents much larger than the renainder.

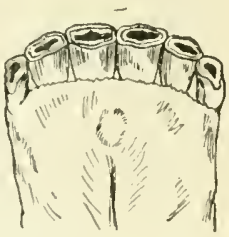

Five-year-old Mouth. All the Permanent Teeth up, but the Corners not fully de. veloped, the blazk marks being largest in the two Centrals.

imperfect or shelly. It will be seen from the foregoing that it is really very easy to distinguish youngsters from one to five years old by the number of the teeth, temporary or permanent,

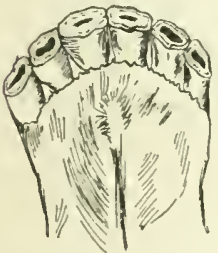

Six-year-old Mouth. The Corners fully developed and the two Centrals be. ginning to lose their black marks.

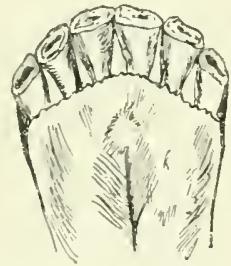

Seven Years Old. The black marks fast dicappearing from all but the Corner Teeth. (Compare with Six and Five-year-old Mouths.) The Teeth are longer and Interdental Spaces wider.

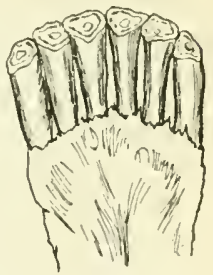

A very Old Mouth. Teeth long. Tables triangular. Interdental Spaces in. creased. Infundibu. lum gone and only an irregular mark in centre left, Jawbone narrower.

yet there are many experienced coachmen and horse-keepcrs who do not possess this knowledge. When the incisors first attain their growth they have a slight concavity on the crown, 
which is filled up with black-looking material ; this will be seen in the foal's teeth as well as the permanent, but is much more marked in the latter. These concavities with their contents disappear with wear, for it must be borne in mind that horses' tzeth keep on growing all their lives. As the central pair are the first to be developed, they soonest lose the markings; the process does not generally occupy longer than a year, making the six-year-old mouth differ from the five by having the marks gone, or nearly so, from the two central, while the outer laterals have grown up level with the rest. At seven years the second pair have lost their markings, and only the last or outer laterals retain the mark, which after this time gradually disappears, the horse being aged seven or over. These remarks apply equally to mares and geldings, but the latter have four additional teeth, called tushes or tusks, which have no particular function to perform, and are, according to the evolution theory, the last link with the past, when horses were tapirs or something else. The tushes ${ }^{1}$ come through at about the same time as the last pair of incisors; they are at five years old on a level with the nippers for length, and the inside surface is concave while the outside is convex, the point being sharp. The age of geldings when past seven is more or less ascertainable by the shape of these teeth, which is not the case with mares, in whose mouths they are often found in a rudimentary state. As age advances, the point of the tush loses its sharpness, and the inner surface its concavity, so that it becomes round. In some old horses it remains rounded and blunt, while in others the position changes, till, in extreme old age, the tushes point outwards like those of the boar.

With very little trouble an amateur may learn to distinguish between colts up to five years old; it is the varying degrees of attrition and retention of the black marks that deceire any but experts. We have known a very old horse retain a five-yearold corner tooth, as the outer laterals are called, because the tooth has never been fully developed or received any wear from

1 They are more developed in entire horses. 
the corresponding teeth in the upper jaw. Not a few disputes as to the age of horses occur even among the best veterinary surgeons; hence it may be concluded that the signs herein described are not absolutely to be trusted, though the great majority of horses cut and also wear the teeth in the manner indicated.

There are a good many devices for making colts look more than their age, such as extracting the temporary teeth, but no one sign should be trusted. The general development and the time of year should be taken into consideration. For convenience of reckoning all half-bred horses date their birth from Nay $\mathrm{I}$, and thoroughbreds from January $\mathrm{r}$. The latter may be and are foaled at all times, but the intending racer gains advantages by being born early in January, and getting classed as a yearling or two-year-old with others born later in the same year. The half-bred horse, by which we mean everything but the racehorse, is reckoned from May I, because the majority of foals are dropped between April and Midsummer, and May is approximately the birth month of them all-the colt has all the summer before him at grass, and his dam makes plenty of milk, and enjoys idleness at a time of year when her owner can keep her cheapest. It is also the period dictated by nature.

Assuming that the difference between a foal under a year and a two-year-old is recognisable, let us now parade a two or three, a four or five year old horse in the early spring when buying in for the summer work. The two-year-old will have a complete set of incisors, but no tushes, whether mare or gelding. They are not large enough, and are too much alike, and he must be rejected. The three-year-old will have two permanent teeth in the lower jaw very much larger than the others, quite sufficient in itself to prove that he is a three-year-old. 'The four-year-old will have four permanent teeth in the lower jaw, and a milk tooth at each end, looking so very small by comparison with the rest that one can hardly be pardoned for mistaking a four-year-old mouth when once seen. The five-yearold will have a full mouth, all the black marks showing, and the corner nippers not fully developed. 
We have commenced our examination of the horses in the spring, and they are then said to be 'rising' $2,3,4,5,6$, or 7 years old, as the case may be. After the month of May, they are said to be $2,3,4,5$, or 6 'off.' These terms are rather confusing to beginners, but they are expressive. For six months after a birthday, a horse is so many years 'off,' and for the next six months he is 'rising' so many years.

In the early years of a horse's life, the seller is anxious to make him look older, and in his later years younger. The latter process is called 'Bishoping,' from the fame of a certain scoundrel by that name, who reduced it to a fine art. Old horses are palmed off as seven years old by digging out the crowns of the corner nippers, and plinging them with black composition so as to represent the original infundibulum. Practitioners of this art do not commonly take the same trouble with the upper incisors, which should be looked at and compared if there is any doubt. Some horses, whose lives have been spent largely at grass, will retain a very youthful set of teeth up to a dozen years old, and these are usually the subjects chosen for 'Bishoping,' though some bold operators take the trouble to cut and rasp down the long teeth of a very old horse, preparatory to stopping them. These tricks are not so often ${ }^{1}$ practised now as formerly, when certain gipsy-bred copers used to make a regular business of 'setting' broken-winded horses, and 'puffing the glims,' and 'Bishoping' old screws, exciting them with torture, and making them carry their heads up, and cock their tails by ' figging.' ?

1 Since the earlier editions of this book were published there has been a recrudescence of 'Bishoping,' which has been reduced to a fine art by the invention of tools as well suited to the work as the burglar's jemmy or skeleton keys for housebreaking.

2 'Puffing the glims' means the introduction of a fine tube into the hollow above the eye, and blowing out to make it round and full. 'Figging ' is the introduction of ginger into the rectum just before showing the horse ; the irritation and excitement produced by it lends for the time an animation which may be mi-taken for the fire of youth. 


\section{DISEASES OF CATTLE}

Although we do not profess to give anatomical details in this work, it may be of service to describe briefly the process of digestion in the ox, as illustrated by the accompanying diagrams. The $o x$ is liable to choking by attempting to swallow a piece of turnip or other food too large for the gullet. A whip-handle is often used by the attendant to force the food down in such a case, and sometimes serious injury is done by the violence used. A probang and gag should always be at hand where cattle are kept.

From the gullet the imperfectly masticated food passes into the rumen, paunch, or first stomach, where it is macerated for a time before being returned for a further grinding (chewing the cud). Sometimes the rumen is overloaded, and the animal moans, the flanks swell, and much danger exists. The probang has to be used, or perhaps the stomach-pump, after injecting fluid to dissolve the food, or in some cases it becomes necessary to make an incision and to remove the food by the hand. A more easily dealt with trouble is tympanites or hoven (p. I62), which results from an excessive evolution of gas in the rumen. Besides the treatment detailed in that article, a simple and often effectual remedy is a dose of 2 drachms of chlorinated lime mixed with half a pint of water. The function of the second stomach (reticulum or honeycomb) is to further prepare the food for re-mastication. After the second chewing the food passes into the third stomach, or maniplies, which consists of a series of leaves which triturate the food into a state of division, and thence into the fourth stomach, in which true digestion takes place. 
The order in which the food is received into the various stomachs is not very clear, but from the slaughter of an animal in the act of feeding Professor Simonds infers that the order stated above is the correct one. One experiment upon one kind of food is, howerer, scarcely conclusive, as pocket-knives and bunches of keys have been found in the rumen after months and years, and there is reason to suppose that the stomach of the cow had some power of selection like that

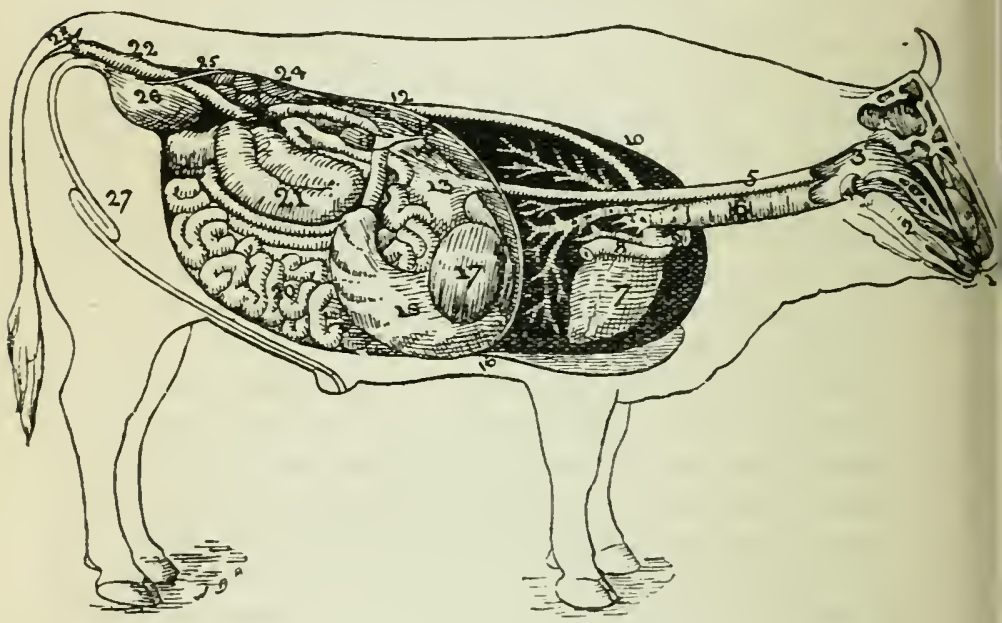

1, Nouth ; 2, tongue: 3 , pharynx; 4, epiglottis ; 5 , assophagus; 6 , trachea, or wind. pipe; 7 , heart ; 8 , anterior vena cava ; 9 , anterior aorta; 10 , posterior aorta ; 11 , bron. chial tubes; 12 , diaphragm; 13 , cardiac orifice ; 14 , liver; 15 , rumen, or paunch ; 16 , reticulum, or second stomach : 17 , omassum, or third stomach ; 18 , abomassum, or fourth stomach: 19, gall bladder; 20, small intestines; 21 , large intestines; 22 , rectum; 23 , anıs; 24 , kidney; 25 , ureter; 26 , bladder; 27 , penis, or urethral canal.

commonly accorded to the pylorus or intestinal opening of the stomach in man, which rejects a foreign body time after time and finally allows it to pass.

Constipation.-Horned stock are subject to constipation when stall-fed or yarded in winter upon dry food. The bad hay of the summer of $18 S S$ will he long remembered by stockowners and vetcrinary practitioners for its effects upon cattle. 
It could not be sold, and had to be disposed of upon the farm, consequently the farmer had to feed his store stock if he had any, or, still worse, his cows with it. When flavoured with some of the popular condiments animals would eat it, only to become distended, dyspeptic, and 'dung-bound,' or 'fardel-bound,' as the expression goes. 'Clue-bound,' 'the grunts,' 'stoppage,' \&c., are varieties of popular description all having much the same meaning, and referring to the impaction

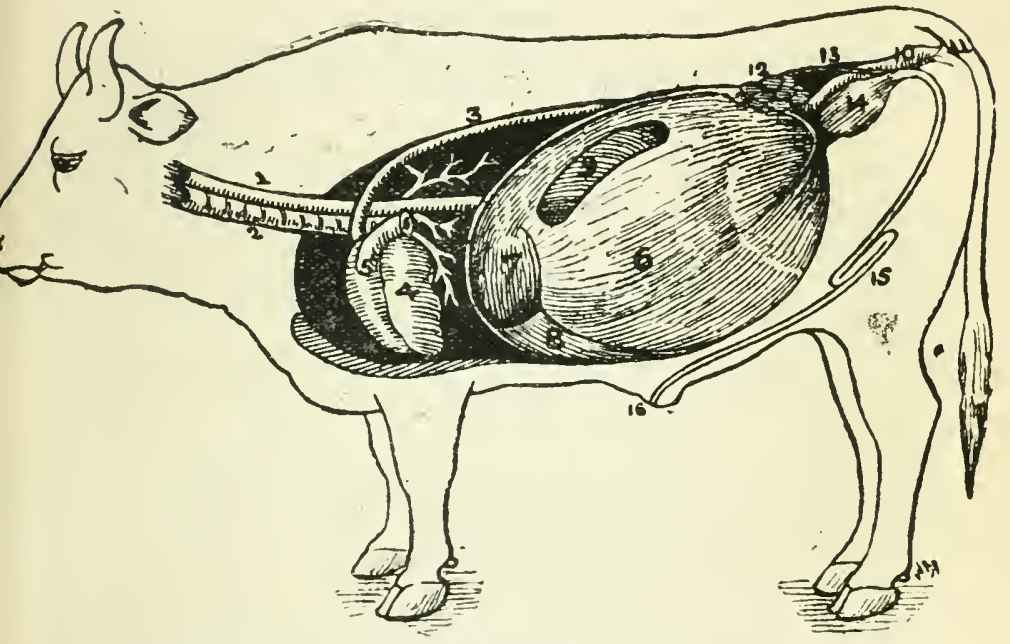

I, Fsophagus; 2, trachea; 3, posterior aorta ; 4, heart ; 5 , posterior vena cava ; 6 , rumen, or paunch; 7 , reticulum, or second stomach; 8 , abomassum, or fourth stomach ; 9. rate; ro, rectum; II, anus; 12, left kidney; 13 , left ureter; I4, bladder; 15 , penis ; 16 , sheath.

of the third stomach, or omassum. The first stomach, or 'paunch' as it is called by the butcher-'rumen' by the anatomist -is very seldom the seat of impaction; its contents are generally soft and fluid, and it is more liable to distension by gases than to become dry and solidified. Nevertheless, such a condition is occasionally met with. The disease or derangement of functions with which we have at present to deal concerns the third stomach, which is called 'omassum,' 
' fardel,' 'maniplies,' and many other names, and contains an arrangement of leaves, between which the partially digested food is always found in a comparatively dry condition in the slaughtered animals at the shambles. The surface of these leaves taken together is very great, and they are covered with papillæ. The attachment of the membrane is very delicate, and can be rubbed off with the finger and thumb. This being the case, it needs but little irritation from retained and dry ingesta to produce very serious local and afterwards constitutional disturbance. The causes are generally said to be chills, sudden changes of temperature, want of water, greedy feeding after enforced abstinence, dry and innutritious food, eating of acorns, \&c. These may be among the causes, but such cases are not infrequent with stall-fed animals with a tank of water beside them and a warm shippon or byre, so that any form of indigestion may lead up to it, and what more likely than indigestion among animals living such an artificial life as a tied-up cow or a fatting bullock?

To diagnose this complaint is not always so easy as might be supposed, as it is sometimes accompanied by a kind of diarrhcea of a very offensive nature. If there is obstinate constipation, a drum-like condition of the abdomen, staring coat, dull, heavy expression, loss of cud and of appetite, and at each expiration a grunt is added, it will be pretty safe to assume that impaction is the cause. There is also tenderness over the region in some cases, and knowing ones think they detect it by the sudden flinching of a bullock when pinched on the back above the shoulders. This feature is an assistance to diagnosis in some other diseases, especially lung and liver disorders. When the diarrhceic symptum presents itself a careful examiner will find that the offensive excreta are a thin liquid running through more hardened matter which adheres to the sides of the bowels. Such a condition is known to stock-owners and veterinary surgeons as 'bound forward,' which is literally the case : a fermentative diarrhoea is going on behind as a result of plugging in the anterior part of the digestive apparatus. 
Treatment.-This must, of course, be directed to the immediate removal of the long-retained and fermented food. $A$ bold aperient should be given, although signs of inflammation are imminent and the temperature rising, for without dislodging the impact recovery is impossible. A good dose is the following :

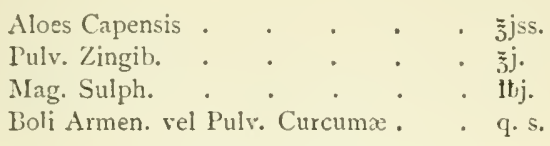

Ft. pulv, pro haust.

To be mixed with a quart of boiling ale or gruel, and given while still warm.

The cow-shed or farm premises do not, as a rule, afford much facility for mixing up medicines, and the above appears a clumsy way of giving a drench; but most hinds are accustomed to the powder-drenches, and prepare them by pouring the boiling liquor upon the powder in a jug, stirring the while with a small faggot-stick.

Old-fashioned folks still prefer a horn for administering medicines to stock, but a champagne bottle is much more convenient. The former is easily slopped over and still more readily knocked over, by the patient, while the danger of breaking the neck of the bottle in the animal's mouth is so slight that we cannot call to mind an instance in an experience of many years.

A very useful drenching bottle is that supplied by Messrs. Day, Son, \& Hewitt.

If the above dose does not move the bowels in ten or twelve hours, large quantities of hot water should be horned down (if the animal does not drink it), and $2 \mathrm{lbs}$. of treacle mixed with a pailful of hot water, and given every two hours. Some practitioners depend entirely on repeated doses of linseed or castor oil, but it is a good plan to alternate the treacle and oil doses every two hours, giving at least a pint of good linseed oil each time. Castor oil is not so beneficent in its action, and constipation is more likely to recur at no 
distant time, while linseed oil is food as well as medicine, and many animals will eat it poured over their chaff or bran. We know a very large establishment where each bullock has a pint in this manner twice a week, and the herd has been remarkably free from digestive derangement.

If there is prostration with the stoppage, evidenced by cold horns and legs and hanging head, stimulants and cordials can with advantage be added to the foregoing. If the medicine is to be sent out as 'drinks,' a good combination is as follows :-

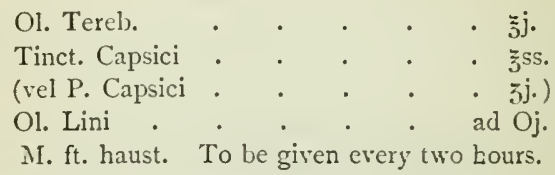

The measure of purgation necessary will be best estimated by examining the freces, which the stockman should be directed to put by if hardened, darkened, slimy lumps are observed surrounded with loose dung, and the bullock has since passed dung that is all soft; then the aperients should at once be stopped, and nourishing cordials given with gruel, and bran mashes or other soft and easily digested food; but a little hay must be allowed, as a sick animal can scarcely recover his cud without a small allowance of long food. The following, given alternate days for a week, will greatly facilitate recovery, and, if a cow, restore the secretion of milk; with a steer or fatting-beast it will enable him to pull up again and lay on flesh.

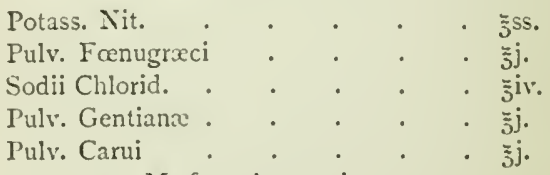

M. fi. pulv. pro haust.

To be mixed with two or three pints of ale or gruel, and given warm at night.

$\Lambda$ gradual return to the ordinary diet should be enjoined, and a lump of rock-salt put in the manger to lick at wili. 
If the beast has not been treated at an early stage, and is in butcher's condition, it is better to slaughter him, as the carcase is quite fit for food, and if the owner has any doubts it will prevent a loss by obtaining a qualified reterinary surgeon to certify the carcase.

For the relief of constipation produced by bad hay or long-continued dry fodder, we advise several doses, at intervals of a day or two, of the following as a suitable drench-not a scientific, but often a successful combination:-

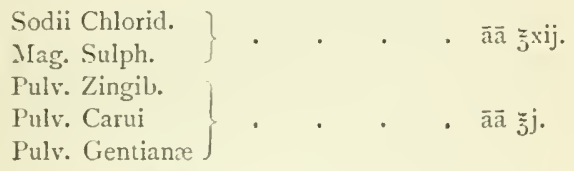

II. ft. pulv. pro dosis.

To be given with a pound of treacle and a quart of hot beer or thin gruel alternate nights till the desired result is obtained.

A good cattle-spice will after this be of great assistance; most of the popular cattle-spices are of value as aids to digestion, especially after an illness in connection with the digestive apparatus.

Scouring, or Diarrhœa, in Calves.-This malady is the cause of more losses among calves than even the dreaded anthrax, as the former is, like the poor, 'always with us,' while the latter makes occasional visits of more lasting impression. It is called by a variety of names in different districts; among the commonest are scours, skit, white scour, black scour, \&c. White and black scour indicate different forms of diarrhcea, or different stages. The most frequent form of scours is that found in artificially reared calves, and is of the white kind. It is undigested, or curdled milk, in the fourth stomach, and is not, as is supposed by some, due to inflamed mucous membrane. Professor Gangee says : 'In the many cases I have examined there was usually a peculiar pallor, or indications of checked function, in the fourth stomach and intestines. It 
is the mass of half-curdled milk in these organs, and the emaciated appearance of the tissues, which may be regarded as characteristic of diarrhoea in suckling quadrupeds.' This opinion being supported by the best observers, there should be no insuperable difficulty in the treatment unless constitutional and hereditary disease is a concomitant. The old-fashioned remedy of opium and chalk, and cordials such as aniseed and fenugreek and cumin, have much to recommend them, and will bear scientific examination-indeed, it is a puzzle to modern scientists how the illiterate class of men who have for ages practised with considerable success among animals could have found out remedies without knowing the why and the wherefore-seeing through a glass darkly, yet attaining their object. Of course they were illumined from time to time by medical men and others of better education; but practitioners of that class who remain resent fresh ideas, and are dying out in competition with the better-trained veterinary surgeons of the present day.

It is good practice to give a dose of castor oil, from half an ounce to two ounces, before resorting to any astringents. There are matters to be got rid of, and the after effects of castor oil are distinctly astringent.

Infusions of astringent barks, such as oak and elm, will often answer the purpose where the chalk and opium seems too clogging, but the admixture of a fourth part of limewater with the milk has the recommendation of being cheap and easy of administration, as well as effective. Care should be taken that the milk given to the calves is sweet. The following is a very good mixture for general use among calves :-

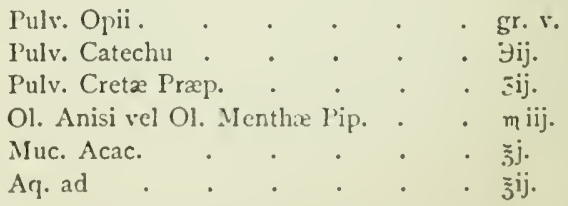

M. To be given in gruel and repeated next day if necessary. 
Or-

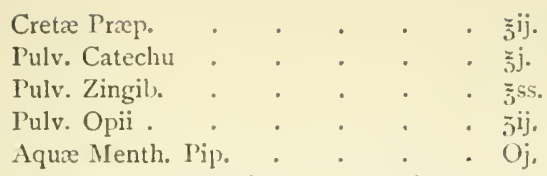

Dose : $\check{3}$ j. night and morning.

Tinct. chloroformi et morphinx co. is preferred to opium by many advanced practitioners. Another scour mixture which is often found serviceable is the following :-

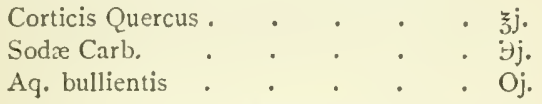

Infuse for an hour. Dose: Two tablespoonfuls night and morning.

Where some amount of blood is passed with the excreta a bismuth and acacia mixture may be prescribed, preference being given to the subcarb. or trisnit. over the liquors.

Jaundice, familiarly known as the 'yellows,' is of frequent occurrence among cattle as compared with horses. It is most often seen a few weeks after calving. The yellowness of the skin and membranes occurs as in other animals, but is not so easily recognised in good 'butter cows,' as their skin is normally yellow. There is an unthriftiness about the coat and a rapid loss of flesh and failure of milk, nausea, loss of appetite, imperfect digestion, pain when right side is pressed, irregularity of bowels; sometimes constipated and at other times frothy dung is passed, containing 'bubbles.'

Treatment.-One of the most successful cow-doctors of the old school placed his faith in the following; it is not at all orthodox, but he obtained much credit for it :-

\begin{tabular}{|c|c|c|c|c|c|}
\hline Calomel . & • & - & • & • & \\
\hline Aloes Socot. & & - & • & - & \\
\hline Mag. Sulph. & & - & $e^{\circ}$ & • & . \\
\hline Pulv. Zingib. & & . & - & - & \\
\hline Pulv. Curcumæ & . & . & . & . & \\
\hline
\end{tabular}


This was given in a quart of hot ale, and forty-eight hours later the following dose was to be administered on alternate days for a fortnight :-

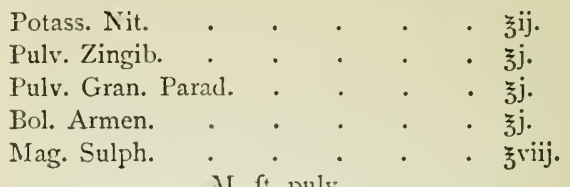

To be administered in hot ale. We cannot follow the science of this treatment, but have found it answer in a larger number of cases than any other. The following may often be found useful in these cases :-

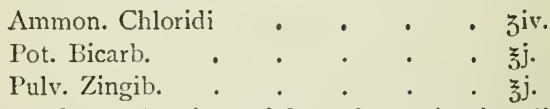

M. ft. puls. One to be given night and morning in a little gruel.

Tympanites, or Hoven.-After months of stall-feeding or dry food in yards, horned stock welcome the change to green food with greater joy than discretion, and it is generally at this time of year that tympanites is met with. It is called, in various parts of the country, the hoove or hove blown, the blast, hoven, fog-sickness, \&c. This distressing complaint presents no difficulty in diagnosis, as the belly is distended to an enormous sizc. Red clover and vetches more often produce it than other green stuff, but where cattle break their fences and get into young and abundant green food, and overgorge themselves, there is always danger of their becoming hoventhe rapid fermentation and decomposition of the food is the result, and carbonic acid gas and sulphuretted hydrogen are climinated in enormous volumes, and to such an extent sometimes as to burst the rumen before assistance can be obtained. The diaphragm, or midriff, has been known to be ruptured either from the pressure or in falling, and the respiration is invariably short and difficult, through the diminution of the chest rapacity caused by the bulging forwards of the diaphragm. there are of course, degrees of tympany, or horen, and instead 
of an acute attack it will sometimes develop into a chronic state of dyspepsia, the flank being always like a drum and the eructations offensive, instead of being like a nosegay, as in health. If the acute form is to be dealt with, the treatment should be directed to at once diminish the volume of the paunch by giving such chemical agents as will decompose $\mathrm{CO}_{2}$ and $\mathrm{H}_{2} \mathrm{~S}$ into harmless, if not actually beneficial, products, and prevent further fermentation. Of these agents ammonia is the best, and in the form of aromatic spirits it reduces the rolume and acts again in the form of carbonate. It should be combined with stimulants and stomachics to induce contraction of the muscular coats of the stomach. The follow. ing will be found a good draught :-

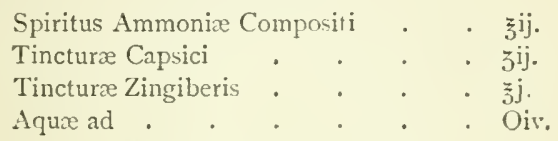

M. ft. haust. statim sumend.

This may be repeated in half an liour if no relief is obtained; and, failing to relieve in an hour, recourse should be had to the trocar and cannula or the hollow choke-rope. As some readers may not be familiar with these instruments, we will describe them. The choke-rope, or probang, when properly made, is about six feet long, composed of coiled wire with a leather cover, and turned-wood or whalebone ends-somewhat egg-shaped-with a cup-like depression in one of them ; through the whole length passes a whalebone or cane stiletto. To use this instrument, the cow must be secured by the horns and held by two assistants if possible. One should hold her head up, by putting his finger and thumb firmly in the nostrils, while the other grasps the tongue or maintains a gag in position, if the operator is fortunate enough to have cne with him. The stiletto should not be withdrawn, but the end of the probang should be oiled and then introduced into the mouth, pushing it steadily over the tongue and down the gullet, till it is nearly all out of sight, when the stiletto should be withdrawn. A volume of gases generally rushes out, and 
the flank falls in like a pricked balloon. But this happy result is not always obtained; sometimes the food persistently chokes up the instrument, and will not be got rid of by repeatedly passing down the stiletto. When this is the case and relief is not obtained, the trocar must be resorted to. This is an instrument about eight inches long, with a point which makes a leech-bite puncture. It is ensheathed in a metallic cannula, with a cup-shaped top, but which does not cover the other end of the trocar, with which the puncture is made. The operation, which is commonly known as 'paunching,' is performed by pushing this sharp instrument right through the left flank into the rumen; the situation to be chosen is between the last rib and the hip, some four inches from the rib; the direction should be downwards, and the force used sufficient to drive home the cannula right up to the cup, withdrawing the trocar and getting out of the way as quickly as possible, to avoid the immense volume of offensive gas which rushes out, extinguishing a candle if it happen to be by night that you are operating. The cannula may be left in for six or eight hours, until remedial agents have reduced or dispelled the gases; and it is very seldom that any trouble is experienced with the wound produced. In cases of emergency an incision with a penknife will often give relief. It is essential to get rid of the offensive ingesta, and restore the digestive functions, and this is best done by repeated moderate doses of saline aperients, with what are commonly called cordials - ginger, gentian, calumba, fenugreek, \&c. Diapente used to be much prescribed, but its composition was probably not so well known to prescribers as to druggists of olden time, and we should not enumerate it among the best remedies. The following is a suitable draught for the convalescent stage, and should not be omitted, as it is a neglected case at this stage which so often develops into a chronic one :-

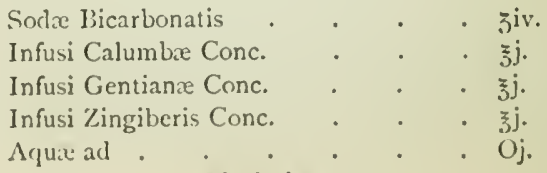


This should be given daily, and about I lb. of mag. sulph., more or less, according to the size of the beast, on alternate days. The remedies should not be discontinued till the dung ceases to show bubbles when freshly dropped.

Chronic hoven may arise from other causes, but is usually the result of indigestion. It has been caused by part of a blanket found in a cow's stomach, or rather in the junction of the stomach, and other foreign bodies are not at all rarely found. In the museum of the Royal Veterinary College are to be seen a bunch of keys, a pocket-knife, an old shoe, and other 'unconsidered trifles' which have been snapped up by cows : we have seen a perambulator apron and a child's pinafore taken out of a cow's paunch, but we have not heard of a baby being lost in that way. As it is impossible to diagnose the presence of foreign bodies during life in the rumen we can only give those remedies which experience has proved to be effectual in ordinary tympanites, or hoven. When time is not of so much importance as in the acute disease, the agents may be given in powder; some veterinary surgeons think they act better in that form. The following may be given twice a week, and will prove a 'thriving' drink for cattle when not dyspeptic :-

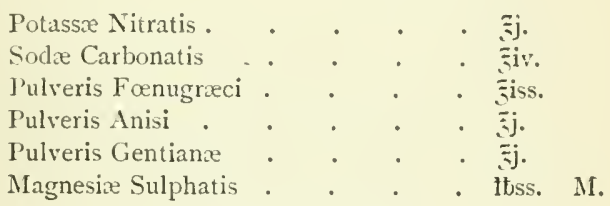

To be given in a quart of hot ale at night.

This dose will be found to increase the quantity of milk in a cow, but must not be continued for that purpose for longer than a fortnight.

' Red Water.'-The cause of this disease is obscure, but it will probably be found to be allied to anthrax, symptomatic anthrax, purpura, \&c. It used to be, and indeed still is, attributed to rank herbage and pithy turnips, but there is no proof to warrant such assertion, and it is far better to confess our 
ignorance. It should be understood that red water is passed by cattle, not because the bladder or any portion of the urinary apparatus is diseased or bleeding (bloody urine being quite another condition), but owing to a blood disease in which the red corpuscles are broken up, and, mixing with the urine, change its colour from a pale yellow to black, or any shade between; depending largely upon the amount of red corpuscles in solution and the degree of dilution with normal uriniferous fluid.

The chief symptom is the sudden change in the colour of the urine, frequently obstinate constipation and always rapid wasting. When pressed over the withers, the cow crouches, and sometimes shows tenderness over the loins as well.

Treatment. - After an interval, in which the advanced school of reterinarians condemned the farriers' methods and recommended 'tonics and stimulants from the commencement,' a return has recently been made to the old plan, as a German scientist has 'discovered ' the advisability of giving a bold dose of aloes and salts, such as our old cow leeches gave 200 years ago. As much as two ounces of aloes is often given with pot. nit. I ounce and mag. sulph. half a pound, or

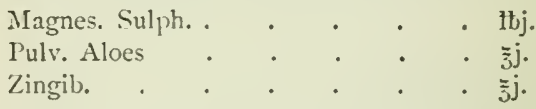

'To be given in not less than three quarts of oatmeal gruel.

Port wine in wine-bottle doses is sometimes prescribed. Perhaps its colour is thought to act homcepathically; we cannot of course say, but our experience of the cow-house is in favour of a witness wherever bottles of wine or whisky are prescribed : the effects are not always appreciable upon the cow, but the fumes inhaled by the attendants (during the drenching process, it is supposed) are often 'extensive and peculiar.'

A tonic stimulant of proved value is the following :-

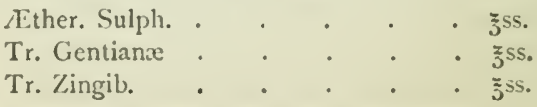

To be given once or twice a day in linseed tea. An aperient must be given at the outset. 
Care in feeding, especially frequent changes of food, must be attended to. If the animal will not eat, linseed and oatmeal must be given in the form of gruel, together with milk and raw eggs. Fresh air, but an even temperature should be kept up if possible. No diuretics or astringents are to be used, nor any stimulants applied to the loins.

Suppression of Milk, if traceable to indigestion, is best treated by a change of food and three doses of cordial aperient as follows, allowing an interval of forty-eight hours between each :-

$$
\begin{aligned}
& \text { Potass. Nit. } \\
& \text { Pulv. Anisi } \\
& \text { ", Carui } \\
& \text { ", Capsici } \\
& \text { Mag. Sulph. }
\end{aligned}
$$

ziij.

j.

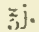

引.

zx.

To be given in a quart or three pints of good ale at bloodheat.

Cow-pox is a contagious disease accompanied with a febrile state and eruptions upon the teats. It has a period of incubation of from six to nine days. First a pimple or papule, which fills with watery fluid, and afterwards matter or pus; it then breaks and forms a scab, which falls off and the surface heals up. In more scientific language it may be divided into periods of incubation, invasion, papulation, resication, pustulation, desquamation, and resolution. Its importance arises from its effects upon the human subject. Vaccination is said to have originated from the observation of milkers escaping small-pox after having cow-pox. A minority of medical men and a large number of laymen will not admit the claims of vaccine as a prophylactic against small-pox, but consider the danger of transmitting diseases from one child to another more than counterbalancing its benefits, if it have any. This objection might be overcome, if only Government-stamped calflymph were permitted to be used, and inoculation with any other lymph rendered penal. Another objection is the not 
entirely disproved opinion that the vaccine disease of the cow was originally produced by that most offensive disease of the horse commonly known as 'grease.'

The eruption on the teats, which characterises cow-pox, is accompanied by a certain or uncertain amount of febrile disturbance, sometimes so little that nothing is observed amiss but for the eruption, the scabs of which are often enough rubbed off the teats and into the pail, to be rescued again by the strainer before the 'pure country milk' is sent off to London. Every precaution is taken by the great dairy companies of London, who send veterinary surgeons from time to time to the farms to inspect both the cows and the milkers, and the supply is instantly stopped where any disease of the kind is known to exist among milkers or milked.

The treatment of cow-pox consists in the administration of a few doses of a saline medicine such as is prescribed below, together with fomentation and application of ung. althææ daily to the teats, unless a tendency to ulceration is shown, when an astringent ointment, composed of 20 grains alum. exsicc. to I oz. of lard, should be used, or a lotion of boracic acid $5 \mathrm{j}$. to $5 \mathrm{x}$.

$$
\begin{aligned}
& \text { Potass. Nit. . . . . . } 3 \text {. } \\
& \text { Potass. Bicarb. . . . . . そ zij. } \\
& \text { Pulv. Anisi . . . . 3iv. } \\
& \text { Mag. Sulph. . . . . . కviij. }
\end{aligned}
$$

Ft. dosis. Alternate nights in thin gruel.

The usual form for making ung. althææ is as follows :-

$$
\begin{aligned}
& \text { Ol. Palmx . . . . . lbss, } \\
& \text { Resin. Flav. . . . . Hbjss. } \\
& \text { Ceræ Flav. . . . . . ttij. } \\
& \text { Ol. Lini . . . . . Ibix. } \\
& \text { Olei Myristic } x \text { et Ol. Rosmarini . . q. s. }
\end{aligned}
$$

Owners should be advised not to sell the milk, and to keep the animals affected apart from the rest, not allowing the same attendants to minister to their wants, nor using the same tools or utensils.

Cow-pox of late years has shown itself in a very mild form, 
as is the case with some other animal plagues that once were virulent; but we must not be sanguine as to its ultimate disappearance, as many diseases have a knack of coming into fashion again in a very pronounced form, just as everybody has learnt to regard them as of no consequence.

Milk Fever.-By the name of milk fever, parturient apoplexy, dropping after calving, and the drop, there exists an affection peculiar to recently calved cows. They may be attacked within two hours of parturition, or the disease may appear as late as nine days afterwards. The second or third day would appear to be the most dangerous.

The pathology of this malady is still a matter of doubt, inasmuch as one must read the home and foreign veterinary journals from week to week to keep up to the fashion. To be as brief as possible we may say that, until about fifty years ago, it was regarded as a parturient fever in some way connected with the milk secretion, and it was noted that deep milkers were the most frequent victims. Also that a heifer with first calf was never the subject of the malady, but that the third and fourth calving held more risk than previous or subsequent periods. With the employment of the clinical thermometer it was found that there was no 'fever' in the sense of increased temperature, rather the reverse, the temperature being lower than normal at the commencement of the disease, and often throughout, when no complications arose and the case was not of very long duration. The nomenclature seemed to require revision in view of this fact, and the prominent symptoms of apoplexy or brain pressure and parturient apoplexy were deemed a scientific description and an attractively long name with which to prattle learnedly to the illiterate. The professors sought for lesions in the neck portion of the spinal cord, and examples, changed by the action of the spirit in which they were preserved, were shown round to the classes. We were expected to see ruptured vessels, or at least the extravasated blood, which had of course become yellow under the influence of S.V.M. 
This theory of engorgement, or actual rupture of bloodvessels of the brain and spinal-cord, was a very serviceable one, as medical theories go, inasmuch as it served two generations, while every one was striving by empirical means to find a cure. None did. Various methods of treatment, to be presently described, have been for a time popular, and a large measure of success claimed for them for a time. During the last few years a complete change has come over the opinion of the profession since one Schmidt hit upon the theory that milk fever was due to a toxin developed in the udder, being in some way related to the colostrum ${ }^{1}$ found in milk at the time of calving, and that this material, taken into the circulation, acts as a narcotic poison, paralysing the brain and the whole body more or less, according to the quantity absorbed and the individual susceptibility. There is much plausibility in Schmidt's theory; in the depression of the temperature ; in the paralysis, from which some animals completely recover, with or without treatment; a condition of things quite incompatible with the apoplexy theory, for when paralysis is so caused recovery is slow and gradual. The greatest proof that can be adduced in favour of this, the latest pathology of milk fever, is the greater number of recoveries under treatment based upon war with the toxin.

Symptoms. - Uncertain movement of the hind limbs, paddling the hind feet, an appearance of giddiness when standing, and presently going down and there remaining. In mild cases very little more than paralysis of the hind quarters, and inability to rise, may be observed, and this may either pass off or pass on to something very much more serious. The animal becomes insensible, swings its head round to its side, or, keeping it there, lapses into complete coma, from which

1 Colostrum is a yellow substance found in milk just after parturition, and its office is that of a gentle aperient to clear out from the calf's intestines the accumulations of the latter part of pregnaucy or life in the womb. It makes the milk very high-coloured, and is known to farmers as 'beestings' or furst milk, from which custards are made, or a something rescmbling them, but without eggs. 
she may almost suddenly recover, or die with some complication, as pneumonia. The pressure or effect of the toxin varies much in different animals and on different sets of nerves. One will be quite 'blind,' as it is called, because insensitive to objects or touch upon the eyeball. Another, whose pneumogastric nerves are much affected, will make a roaring noise in breathing, and there will be complete suspension of the bowels' action and that of the bladder ; indeed, many die, from interference with the innervation of the heart, of heart paralysis. These are the subjects which are left well overnight and found dead in the morning. All sorts of ideas are held as to the contributory causes. Teterinary surgeons as a body disapprove of high feeding previous to parturition, and point to a lower average mortality where a hay and oat-straw diet and a purge or two before calving are adopted. There are as many farmers who advocate low diet on the one hand, as there are those who believe that cows at grass, full of rich blood, are least likely to 'drop.' The writer has certainly seen all the different systems tried and all fail, while each advocate was perfectly confident until his time came to have a run of milk fever cases.

Treatment.-A bold aperient dose, such as :

$$
\begin{aligned}
& \text { Aloes Socot. . . . . . } \bar{j} \text { j. } \\
& \text { Mag. Sulph. . . . . . 气 气xij. } \\
& \text { Theriacæ . . . . . thij. } \\
& \text { Aquæ Bullientis q. s. ut fiat haustus. }
\end{aligned}
$$

The rectum should be unloaded with the hand (previously greased and introduced slowly), and half a gallon of soapy water, at about $105^{\circ}$, injected immediately after.

Next apply mustard, or, better still, a hot liniment, to the poll, down the sides of the neck (not on the top), and over the loins. The following is a suitable liniment, of which half a pint should be used at one dressing, and repeated next day if desirable :-

$$
\begin{aligned}
& \text { Ol. Terebinth. Rub. . • . . 亏iv. } \\
& \text { Ol. Sesamæ . . . . } z^{x} \text {. } \\
& \text { Liq. Ammon. Fort. . . . . 亏 } 3 \text { ij. } \\
& \text { Aquæ Dest. . . . . . 引vj. }
\end{aligned}
$$

M. ft. linimentum. 
As a stimulant, whisky is very popular, the more liberal owners giving a whole bottleful at a time and waiting till next day. There are cowkeepers who swear by the remedy, though we have often seen it fail. A better stimulant is-

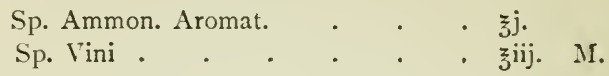

To be given in a pint or more of cold water, and repeated every four or six hours. The attendant should be cautioned about giving medicine to cows in this condition, as many are killed by the drenches going 'the wrong way.' We have seen several ounces of ginger and other insoluble drugs in the bronchi of dead cows. If the nozzle of the bottle or drenching-horn is ntroduced into the mouth for a few seconds before attempting to pour down the drench, the animal is less liable to accident, as the involuntary muscles are prepared for something to come, though the cow may be nearly or quite insensible. This may be questioned by theorists, but men who have seen a lot of cattle practice will agree that it is true.

Chloral hydrate has been much employed, and has given very satisfactory results. An ounce given in a pound of treacle, followed every four hours by the following :-

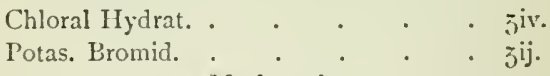

\section{II. ft. pulv.}

is often successful. How it acts has not yet been satisfactorily explained.

In former editions of this work the custom of slaughter has been alluded to, and the greatest authority of the day quoted for the safety of carcases as human food, if killed early and without elevation of temperature or administration of drugs. There is still no proof that the flesh of such animals is not good for food, and the writer has eaten portions of many cows slaughtered by his order during the malady, and observed families so fed, but never detected any ill consequences whatever. But, authority having still more force in the world than either reason or experience, we advise all concerned not 
to slaughter for food, but to attempt treatment. There is such a craze against the poor old cow as the author of tuberculosis while living, and of enteric, and everything else nearly, when dead, that fair play cannot be got for a man who sends a carcase to market that has not been killed while in perfect health.

Cows may have milk fever twice and even three times, but it is best to dry off and fat an animal that has once had it, unless indeed she be one of those cows that will never put on any flesh.

Schmidt's Treatment is directed to the neutralisation of a toxin existing in the mammary glands. A solution of iodide of potassium is the agent employed as calculated to set free iodine with the minimum of irritating effect. It is introduced by means of a syringe, whose nozzle passes into the udder through the milk ducts of each teat; an equal quantity being discharged into each of the four quarters, which, it will be remembered, are quite distinct from each other, though having a common integument. The dose for one injection of each of the four quarters is half an ounce. More for a very large animal, less for a small Kerry or Jersey, but not much less. A pint of water is not too much when divided into portions.

A Higginson's enema is a suitable instrument, but a special nozzle of not less than five or six inches in length must be fitted, in order to pass up the teats without undue force or injury to the delicate sphincter muscles which close their orifices. Schmidt and all subsequent writers have laid great stress on the importance of perfectly aseptic appliances for this work, or septic matter introduced into the mammary gland will be most disastrous. Chemists need not be told how to accomplish this with such a choice of antiseptics. We prefer a solution of chinosol, as one need use no heat for rubber goods, and a very little soaking in a one-in-five-hundred solution is sufficient. The udder is to be washed with warm water and soap first, then sponged with the chosen antiseptic, ${ }^{1}$

* Recent cases seem to prove that the injection of chinosol has the same effect as potassium iodide. 
and, lastly, the bag is syringed through the several teats with the instrument previously prepared.

About six hours is allowed for this pot. iod. dose to take effect, and at the end of that time, if no very striking change for the better is apparent, another injection is administered of similar composition. After a second dose it is sometimes desirable to give, subcutaneously, a dose or two of caffeine, as there is a tendency to collapse from the iodine which has been absorbed.

Some of the most successful practitioners who have published their experiences during the past few years, have adopted the chloral treatment at the same time, or given half-ounce doses of pot. bromide alternately.

Parturient Fever, as we have previously pointed out, must not be mistaken for parturient apoplexy. It is generally a consequence or sequel of inflammation of the womb, following upon a difficult labour, and treatment should be directed both to allaying the local symptoms and arresting, if possible, the bloodpoisoning which constitutes parturient fever. Unlike parturient apoplexy, paralysis is not a marked symptom, but arching of the back and straining, grinding of the teeth and other common symptoms of pain, together with shivering fits, and a high temperature, clearly distinguish fever from the apoplexy erroneously called milk fever. We cannot be too careful to explain the difference to our clients, or they will believe our decisions to be based upon mere caprice.

Inflammation of the womb commonly shows itself the second day, from that to the seventh or eighth, and parturient fever may develop at almost any time until recovery is assured.

Treatment should be both local and general.

If bran poultices can be applied continuously over the loins, much ease will be given, but it is worse than useless to put on a poultice and let it get cold, or allow intervals for evaporation, when a fresh chill may do more harm than poulticing has done good. A little opium sprinkled upon the 
surface of the poultice helps to allay pain. If the prescriber suspects that poulticing will be but indifferently performed, he had better make up a warm embrocation, such as

$$
\begin{aligned}
& \text { Lin. Camph. Co. • . . . 气̌j. } \\
& \text { Lin. Saponis • • • . ₹ ₹vij. }
\end{aligned}
$$

and order it to be applied two or three times a day.

That liniments and embrocations do act upon distant parts beneficially has long been proved by practical men. Possibly, they act upon the superficial nerves in the same manner that the cold wind acts upon the fresh-dropped lamb, sending from the skin a nerve-current. Every cowman and shepherd knows that a calf or lamb will be more likely to live if carried into the fresh air, and nearly all animals are endowed with the instinct to lick their young clean.

The womb should be syringed two or three times a day with warm water, in which an ounce of the following fluid should be mixed:-

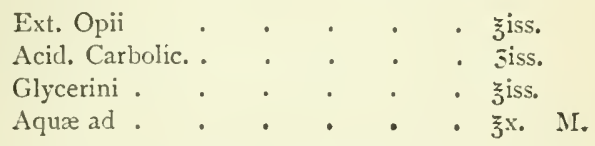

One ounce to be mixed with a quart of warm water and injected into the womb two or three times a day.

Lysol and chinosol solutions are much used by vets. of the modern school.

The following draught night and morning can be recommended :-

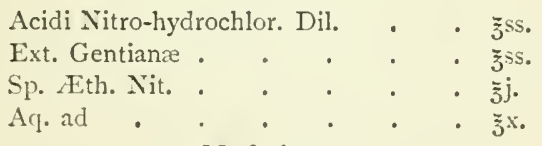

II. ft. haust.

A nourishing diet should be prescribed, and, if all appetite is lost, gruel should be given, of which linseed should form a considerable portion. Or a simple linseed tea may be administered, if the animal will drink it, and if not she may be 
drenched with as much persuasion and as little force as possible, since a nostril wounded with the hard nails of the cowman does not induce appetite any more than the fear of another drenching contributes towards digestion. Cows kept in town dairies become used to the inspection of the public which their owners invite; but many country cows are frightened by the approach of a stranger, and alarmed out of all reason if roughly handled when initiated in the art of taking a drink.

Some good hay should be provided, and, if not eaten, ought to be removed each day. A bullock cannot get the cud again without long stuff, and a sick one should never have food of any kind left in the manger when once it has blown upon it and refused to eat.

Abortion signifies the expulsion of the foetus before it is capable of a separate existence, and usually occurs from the third to the ninth month of pregnancy. It may be occasioned by blows, injuries, exposure to cold, fright, improper food, foul smells, or overdriving. Sometimes the disease commences in a herd of cows, spreading from one to another, and is of a contagious nature, being due to a specific bacillus, discovered by Bang of Copenhagen. It is long retained in the genital membranes, and in most cases it is the best plan to fat off cows that have suffered from the contagious form of abortion.

The symptoms, when abortion occurs in the early months of pregnancy, are very slight, the mother being, perhaps, unaware of the mishap. In the later months, however, there is restlessness, the udder is enlarged and flushed, the vulva injected, the ligaments relaxed, and calving pains occur.

The treatment consists in isolating the cow, destroying the foetus and membranes by fire or quicklime, and thorough disinfection of everything with which the discharges could have come in contact. An antiseptic drink consisting of

Sod. Hyposulph. Acidi Carbolici .

in a quart of thin gruel should be given; the vagina should be 
syringed out with carbolic acid ( 1 in 50 ), or with the following lotion :-

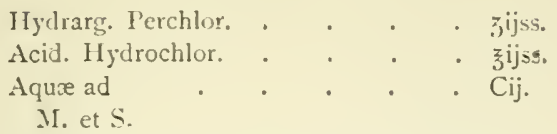

Any other prognant animals in the herd should each receive jss. of carbolic acid in a bran mash once or twice a week, and their vulve should be sponged with the perchloride lotion.

\section{Retention of the Placenta, or Cleansing, is a frequent} trouble in the cow-shed, and, though very disgusting to the casual observer, is not so often a cause of illness as might be expected. The placenta or membranes in which the calf was enveloped in the womb do not always become detached immediately after delivery, especially if the birth is premature, but a portion hangs out of the vagina, while the greater part, perhaps, is firmly adherent to the womb, by reason of the 'roses,' which give it many points of attachment, and should not be roughly torn away; serious hæmorrhage, and even death, has been known to result from pulling violently at the 'cleansing' - as these membranes are commonly called-when it has been attempted too soon; but what more frequently happens, when the operator performs a day or two later, is to break off the part he holds and leave the rapidly decomposing mass behind, without the advantage of outside weight to assist in its mechanical removal.

When the veterinary practitioner is called in, some such bungling as this has often taken place, and he proceeds to dislodge the stinking mass by first injecting copiously with warm water and potass. permangan., or

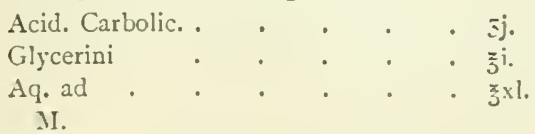

and then, grasping what he can of the placenta by introducing his hand into the vagina, uses gentle traction till sufficient of it be brought forward to wind round a couple of sticks. If he 
finds it very rotten, yet persistent in its hold, he will rest satisfied with winding two or three turns with his pieces of wood, and leave it for another turn next day, meanwhile prescribing such a dose as the following :-
Ol. Terebinth. .
Ext. Ergotæ Liq.
Ol. Ricini ad

II. ft. haust.

Townsfolk, when viewing the beauties of some rustic 'bit' -if we may use artist's slang-are rather surprised to see cows with brickbats or old horse-shoes, hobnailed boots, \&c., attached to the placenta by way of gentle traction. It is certainly more useful than elegant in its operation-æesthetic tastes have not yet permeated the corman.

After difficult labours, the 'shape,' as the external part of the cow's generative organs is called-or 'barron'-though in some districts the latter term has a wider signification-is bruised and swollen, causing some anxiety to the owner (of the vagina as well as of the cow), and should be well fomented with warm water, then dried with a soft old rag, and, lastly, anointed with

Acid. Carlolic. .

Ol. Olive .

Solve.

An injection of pot. permangan. or Condy in warm water, or acid. carbolic. I, glycerin. 2 , and aqua 100 parts warm, is to be recommended. The bowels should receive attention, and any tendency to constipation corrected with repeated doses of ol. lini, which may be improzed with a little ol. anchusæ, and 'nosed' with ol. anisi, vel carui, rel pulegii, or any carminative or cordial. A very useful 'cleansing drink' suited for keeping in stock is :-

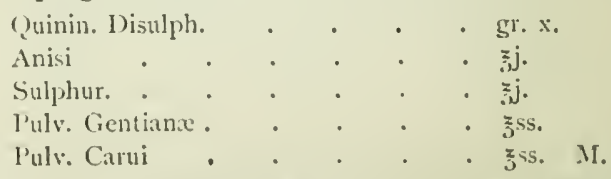

$\mathrm{T}$ r, be given in a quart of thin gruel immediately after calving. 
Inflammation of the Udder.-Mammitis, or 'garget,' is of frequent occurrence in the cow; it may result from external injury, exposure to cold, or irregular and bad milking. The symptoms are at first purely local, but shortly accompanied by fever in greater or less degree, depending upon the sererity of pain, \&c. The secretion of milk is diminished and perverted, being watery, and containing curds; it then becomes yellowish from admixture of pus, and may be entirely arrested. The gland swells, feels hard and hot, is very painful, the veins running from it being much distended. These symptoms may terminate in resolution, but in some few bad cases end in abscess, or even mortification of a quarter of the udder. The udder, it should be explained, consists of four quite distinct quarters or lobes; consequently

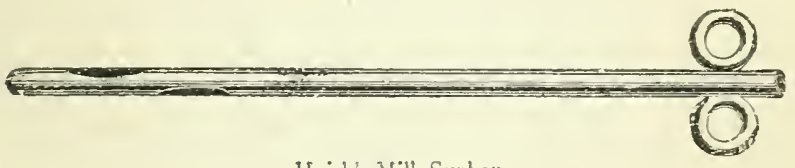

Huish's Milk Syphon.

milk can only be remored from each by its own respective teat. This division usually confines any disease to one quarter.

The general treatment consists in lessening the food and giving a bold aperient, then antacids. Potass. bicarb. in twodrachm doses, twice a day, till the acute symptoms have passed, is generally satisfactory. Local treatment consists in the constant use of WARM fomentation in the form of a linseed poultice, to which may be added ext. belladon. 5iij., and the support of the gland by a carefully arranged bandage, through which the teats must be allowed to protrude. The milk must be frequently but gently drawn off. When the acute pain has passed off, resolution may be accelerated by gentle friction, and, still later, by a stimulating liniment or a mild iodine ointment. Should an abscess form, early incision must be resorted to; once being sure of the presence of matter, do not wait for it to 'point.'

The temperatures recorded by reterinary surgeons in cows suffering from garget are higher than in any other febrile 
complaint, as much as $109^{\circ}$ having been on several occasions noted by good authorities. It generally falls in a few hours.

The thermometer is in this, as in many other diseases, a great aid to diagnosis; every prescriber should carry one ; nor should he forget that in ruminants the natural temperature is two or three degrees higher than in the horse.

Garget is met with in all animals occasionally, but as affecting the cow it has received most attention for obvious reasons. Mares, bitches, sows, cats, $\mathbb{S}$ c., get chills and suffer as nuch; often with fatal results to the progeny. Many a valuable colt has been lost to the owner because the mare would not let it suck a sore udder.

Animals whose young are allowed to suck them are less subject to garget than cows whose calves are taken away immediately they are dropped. The rough brushing of the udder by the head of the young which every one has noticed with lambs induces a flow of milk; while garget is most often met with in cows where bad milkers are employed, or the udders not properly stripped.

There is another kind of garget which old writers called the downfall in the udder, which was accompanied with swelling and pain of the joints of the lind limbs.

In some parts of the Continent garget occurs in a malignant and infectious form, especially among ewes.

'The modern treatment of garget in cows with pot. bicarl), \&c., has been already referred to, but many successful practitioners of the old school put their faith in bleeding and purging, and, if one may judge by results, they are not far wrong. As much as two and even three ounces of aloes with mag. sulph, are given in the following form :-

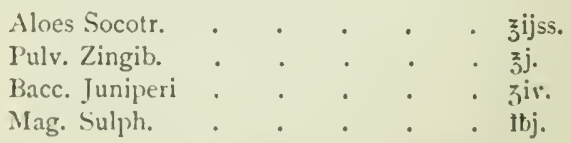

11. ft. haust. To be given in 3 pints of small beer.

For local applications a pound of hog's lard is to be melted 
and rubbed into the bag, and followed by ung. althxx nocte maneque; or, if this fail, the following liniment :-

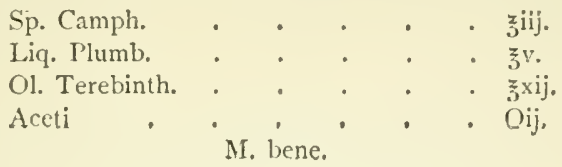

Once daily will be sufficient for this application until the skin shows a disposition to peel.

In cases of garget where a quarter drops out or mortifies, a dressing such as black oils should be applied to the surface of the wound.

Moderns use a strong preparation of carbolic acid, as $\mathrm{I}$ in 8 or 10 of ol. sesamæ or glycerine. The old farriers, who were so fond of acid. sulph. and hyd. bichlor., used the following, which we take from Clater's 'Every Man his own Farrier : '-

Take a pint of linseed oil, and put about a fourth of it into a chamber-pot, then add acid. sulph. $\tilde{z}^{\mathrm{ij}}$. by a little at a time, keeping it constantly stirring with the other hand. When wanted, add by a little at a time ol. terebinth. 3 ij., afterwards adding the remainder of the ol. lini, stir well tngether; lastly add ol. origani $\bar{z}$ ij., tinct. myrrh. $z^{3} \mathrm{ij}$., mix and put into a bottle for use.

There is no doubt about the value of this recipe for the treatment of gangrenous surfaces, but modern pharmacy and convenience point to the use of ol. carbol. or sol. hyd. bichlor. ( $\mathrm{I}$ in 1000$)$.

Bull Burnt is a term applied to a form of gonorrhcea in the cow, and generally yields to simple treatment, though a malignant form of it is met with on the Continent.

A laxative dose in the form of $\mathrm{I} 2$ to $20 \mathrm{oz}$. of mag. sulph. should be given, and the vagina injected with a mild astringent, such as alum $\overline{5} \mathrm{j}$. in aqua $\bar{\jmath} \mathrm{xl}$. ; or Condy's fluid ; or carbolic acid I part, glycerine 3 parts, water 25 parts, 
Extract. hamamelis is the most valued of recent introductions in veterinary pharmacy : one part to three of distilled water injected into the sheath, by means of a syringe, being found most effectual.

Drying-off Cows.-When a cow is to be fatted, she should be first dried off, and this may be accomplished in a very short time with most cows, unless they suck themselves, or each other, when in company.

Dry food is a necessary condition, though some cowkeepers expect a drench to do everything, and will keep a cow at grass for convenience and economy if it happen to be summer time, and we must therefore accommodate ourselves, more or less, to the wishes of our employers, and make them pay for more medicine than would be necessary if our instructions as to dry food were carried out.

There are many ways of accomplishing the object in view, but none so good as the old one of giving bold doses of alumrock. From four to eight ounces should be given as a dose, and the milk not all drawn at a time, while longer and longer intervals are allowed to elapse between the milkings. A good way of sending out a drench of this kind is to give a finc 'nose' as well as colour to it, by the addition of bacc. juniperi zss., coarsely powdered, with instructions to mix it with three pints of boiling water, and give it to the cow when new-milk warm. If the cow is at pasture, or fed on green-meat, the dose should be given about three times in a fortnight, but a couple of doses will be more than sufficient if kept on dry food and short commons.

l'ot. l)itart. is also used for the purpose, in doses of a pound ly itself, or half that puantity with alum $z$ iv. c. bol. Armen. ad colorand.

Bronchitis.-Cattle are subject to bronchitis as a result of cxposure, \&c., and the treatment to be adopted is much the same as that recommended for the same complaint in horses (see p. $S_{\hat{3}}$ ). It is not rare for cows to be killed by getting drenches down 
the bronchi, especially when semi-insensible (sce Parturient Apoplexy, or Milk Fever). Bronchitis may follow such accidents when death from suffocation does not take place. If the drench which has 'gone the wrong way' was a fluid, recovery is probable, but the cordial powders commonly given in gruel, ale, Sc., are apt to prove fatal when any quantity has got into the bronchi.

Rheumatism.-This term is commonly applied to any sort of joint trouble not known to have arisen from external violence. There can be little doubt that articular rheumatism does occasionally affect adult animals, but the chief trouble is met with among the young colts, calves, lambs, and pigs. The swollen joints, the extreme pain and consequent lameness, all point to a rheumatic origin, and by common consent it has been treated as rheumatism. There is, however, pretty conclusive evidence now that the 'joint ill' of young creatures is due to a microbe, for which as yet no pet name has been found. The injection of agents of a bactericidal nature into the immediate region of an affected joint seems, to work wonders as far as recorded experiences afford us information. At the time of going to press with this edition of V.C.P. there are only successful 'treatments' recorded, one of the latest being by Mr. Wartnaby, F.R.C.V.S., of Burton-on-Trent, who uses a 4 per cent. solution of formalin, injecting orer the affected region by ordinary subcutaneous syringe. This looks like the practical application of remedial agents to diseases caused by bacterial organisms, and if our readers are not all prepared to carry out such really simple details, they should at least be up to date when asked about 'joint ills.' Those prescribers who prefer the old order of things will find in the following paragraph something more than a recipe of Clater's: they will be able to reconcile the statements based on experience, and not theory, as to why mait liquors were permissible with cattle when the like would not do for human subjects.

What is generally known as 'joint ill' in cattle has been long recognised as of a rheumatoid nature. No better 
prescription can be quoted than that given by Clater, who prescribed the following with success :-

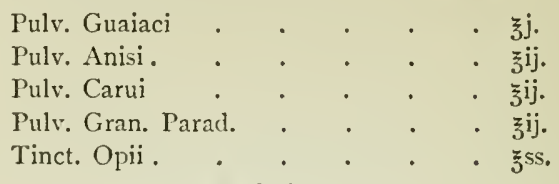

II. ft. haust.

The above dose is to be given in a quart of hot ale or gruel to a full-sized beast. It should be reduced for calves by calculating the proportionate weight of the patients. (See under Diseases of Pigs for external application.)

Modern practice goes to show that fermented liquors are unsuitable in rheumatism and gout in all its forms, and we should prefer gruel to ale; but it must not be supposed that ale is to be tabooed in cattle practice, as experience proves it extremely valuable in combination with purgatives and cordials, and as a tonic after the exhausting diseases of ruminants.

Hoose in Calves. - 'Hoose,' or 'Husk,' is caused by a parasite in the smaller bronchi.

The most effectual treatment is that by intra-tracheal injection of high turpentines with carbolic acid and chloroform, or other substances known to kill the filaria without danger to the host. A strong syringe is sold for this purpose, and a position halfway between the throat and breast of the calf chosen for insertion of the 'business' end of the syringe, which must go clean through the pipe into its lumen, and not merely into it or its lining membrane, for by such imperfect operation abscess subsequently forms, and some young animals have been killed by suffocation. It may be here remarked that the sensibility of the larynx and its lining membrane, which produces almost instant suffocation when anything goes the wrong way, is not shared by the windpipe, and even such powerful agents as carbolic acid in small quantities may be introduced into the lower air passages with but rery little risk.

Either of the following is suitable for calves, and may als $\rho$ 
be employed for lambs if the dose is apportioned to their age and weight. About one-fourth that for a calf, at a rough reckoning, may form a base from which to calculate the dose for lambs.

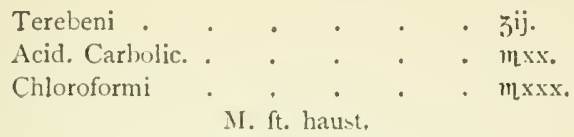

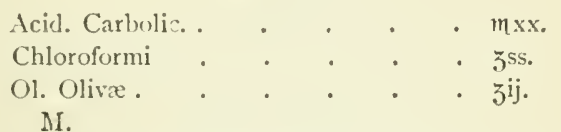

Treatment by inhalation is preferred by many, but serious accidents having occurred the method has somewhat fallen into disfavour.

A perfectly safe and effectual method is the following:Having secured the subjects in a shed, mix a quantity of hot bran and put about a gallon in an ordinary nosebag with buckle and strap ; then pour into the bran a sixth part of the mixture below, stirring the bran and adding a fresh quantum for each calf until six have been done; then throw it away and begin again. About two minutes is sufficient for each animal, and it should be repeated in a week or ten days. A draught as below should be given to each calf.

\section{For the Inhalation.}

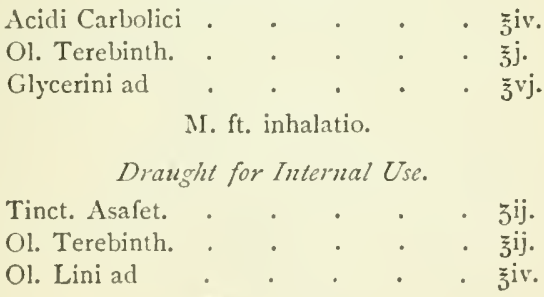

N. ft. haust.

Foot and Mouth Disease.-Murrain, epizootic aphthæ, foot and mouth disease, by all of which terms this affection is known, is a contagious eruptiye ferer, seldom attacking the 
same animal a second time. Though only known in this country since $\mathrm{i} 840$, it had caused great losses on the continent of Europe in $1695,1707,176_{3}$, and later, and in the neighbourhood of Yarmouth, early in 1900, a cordon was established round the affected area, which soon extinguished the epidemic, the animals within the cordon being slaughtered.

Like the cattle-plague and lung disease, it is imported into this country, spreading by contagion, and never arising spontaneously; but, unlike them, it is communicable to man and other animals. Horses and sheep have been attacked from eating food contaminated by an affected cow, and pigs and children have frequently suffered by the milk.

Although murrain is not now very fatal, it entails serious expense from loss of weight and condition, and, among dairy stock, of milk. The symptoms are, first, loss of appetite and general febrile symptoms; if at grass, separation from the rest of the herd. A closer examination shows an eruption of little bladders or vesicles on the lining membrane of the mouth, on the udder, and between the digits. The eruption on the mouth produces a profuse discharge of frothy saliva, and interferes with mastication, in some cases altogether arresting it. Should the eruption spread backward to the pharynx and gullet, swallowing is interfered with.

While the eruptions are forming, the animal champs his jaws, and dribbles ropy saliva until after the resicles break, which occurs in twenty-four hours or less after their formation. These leave ragged places, technically known as ulcers (see Ulcers, p. 272), which sometimes coalesce, and cause much pain and trouble, cntailing loss of condition to the subject, if nothing worse.

l'igs suffer terribly, casting their hoofs, Sc.

'The first symptoms slow themselves between hair and hoof, which part is called the coronet, as we elsewhere point out in connection with the horse's foot (sec Quittor), and in cattle or sheep the greatest amount of swelling and soreness is found between the digits, or claws as they are in some places called. Great lameness and early 
rupture of the vesicles formed upon the coronets are a necessity of the situation; sloughing of the hoofs and death was by no means rare at one time; but this disease, like many others, has undergone modifications, and the few outbreaks that occur give very little anxiety except on the score of infection. No doubt much advance has been made in the treatment, but those who live long enough will see it return in a virulent form, just as influenza and other diseases recur in man and animals. We may hunt for, and perhaps find, a specific bacillus or microbe after thousands of deaths have occurred, but human foresight has not hitherto been sufficient to prevent contagious and infectious maladies from sweeping across Europe, decimating flocks and herds as well as men. The difference between modern and ancient outbreaks seems to be that the facilities for rapid locomotion which now exist render it possible to spread a plague across Europe and America in the time formerly requisite to infect half-a-dozen counties. The doubtful gain as regards live-stock is the tendency to equalisation of values.

The eruption on the udder, if mild, merely renders milking difficult and painful, but it may be so severe as to cause inflammation of the gland. The eruption between the digits, when severe, is perhaps the worst symptom in the disease; vesicles become pustules, and suppuration round the coronet ensues, even to the extent of detaching the hoof. The pain of this complication aggravates the fever, and sometimes leads to a fatal termination; but this virulent form of the disease is uncommon.

So mild have been the last few outbreaks that no treatment was necessary; where it is required, we advise salicylate of sodium as an internal remedy, in doses of from 5 ss. for a lamb to $\bar{j}$. for a full-sized beast. A lotion of alumen exsic. ${ }^{1}$

1 Alum. exsic., whether as a dry powder or in solution, is much more of a caustic, having the effect of drying raw surfaces and hardening shoulder galls and other skin abrasions. But why? What is the chemical difference? If alum. exsic: is only deprived of the water of crystallisation, why should it not be the same thing when redissolved? That its effects are quite different every one will agree who has harl any experience of its use. 
5j. to $5 \mathrm{x}$. to be used for the feet or as a mouth-wash, disguised or coloured as the dispenser may think fit.

The fever and local symptoms are both due to a blood poison. An aperient is generally advisable; for an adult animal a suitable dose is

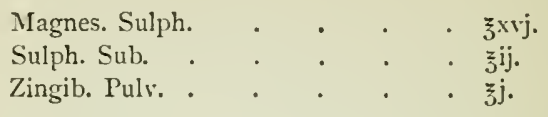

Remember that in ruminants a considerable quantity of fluid is expedient with a purgative; the above should be given in about four quarts of thin gruel. The feet should be washed clean, and then dressed with a mild solution of zinci sulph.

In summer we have seen neglected cases where the feet have been fly-blown and full of maggots, which have had to be dislodged by syringing with a carbolic lotion. The following is a proper formula :-

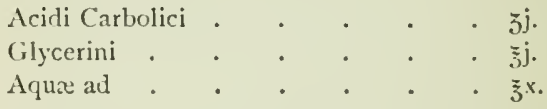

The eruption on the teats requires little to be done; milking must be gentle, and, if found to produce much pain, performed by inserting teat syphons. Some cases may require tonics after, and none can be better than gentian. $5 \mathrm{ij}$., ferri sulph. 5 ij., given in a pint of linseed tea once a day. Hard food, as turnips, may be boiled, and bran-mashes, \&c., given, till the mouth resumes its normal condition.

Pleuro-pneumonia has caused as much stumbling in the veterinary profession as the term has done among laymen. It is a specific contagious disease, and when proved to exist no treatment can be adopted, since the law demands slaughter of the animal affected as well as of those in immediate contact with it. Among the older veterinarians are to be found men who believe in its successful treatment, and there can be no question as to the recovery of some few of the subjects before the present stringent laws were put in force. The battle still rages among veterinary authorities as to the necessity of 
slaughter or the desirability of inoculation. Mr. Rutherford is a warm adrocate of inoculation, and claims to have saved a lirge number of animals ; while Sir George Brown, late of the Peterinary Department of the Privy Council, advocates the 'stamping-out system ' under the compulsory slaughter order.

To diagnose this disease is not such an easy matter, as pleuro-pneumonia of the sporadic or, if we may use the term, accidental variety occurs from time to time among cattle, and is no more infectious or contagious than an ordinary cold or the inflammation that follows exposure and hardship.

The loss to the country from this disease is beyond calculation, and under the new Act it has practically been got rid of, so far as this country is concerned. No doubt in these islands we should have been clear of it long ago but for the fact that the unfortunate owners keep back the necessary information which would enable the authorities to act promptly. The greater liberality on the part of local authorities in matters of compensation now empowered will probably induce more immediate action in future outbreaks.

Pleuro-pneumonia Contagiosa is, as the term implies, inflammation of the pleuræe and lung substance, and is both infectious and contagious. It is characterised by increase of temperature, cough, staring coat, depression, capricious appetite, and segregation from the herd where animals are at pasture. Its progress is uncertain-in some rapid, in others gradually invading the lung substance, giving it a mottled or marbled appcarance when examined post mortem. Its importance as affecting the health and wealth of the nation can hardly be over-estimated. Royal Commissions have been instituted in nearly all the countries of Europe. The real bone of contention among scientists is whether inoculation does or does not produce pleuro-pneumonia or any of its symptoms. Practitioners of undoubted merit and painstaking investigators and statisticians are confident of the immunity given by inoculation, and while unable to give a satisfactory reason for the faith that is in them, they point to what they believe to be results while pure scientists ask for a sign. The controversy has been raging for 
a long time, and local bodies are swayed first one way and then another by the conflicting evidence of experts. We quote from two or three authorities. Professor Williams, Principal of the New Veterinary College, Edinburgh, says :-

The cause of pleuro-pneumonia, in this country at least, is undoubtedly contagion and infection, and these only. My experience of the disease enables me to state that no mismanagement with regard to feeding, housing, or the general treatment of stock will induce an outbreak of pleuro. pneumonia contagiosa. . . I I do not say with some writers that it nowhere originates spontaneously, but I do say that it never originates spontaneously in this country. That pleuro-pneumonia is both contagious and infectious has been proved by direct experiments performed in France, reported by Professor Bouley.

The conclusions drawn by the French Commissioners, as the result of exhaustive experiments, are that-

Pleuro-pneumonia is susceptible of transmission from sick to healthy animals by cohabitation. Twenty per cent. of the animals manifest a resistance to the contagion. Eighty per cent. manifest various effects of the contagious influence. Fifty per cent. are seized with decided symptoms of pleuro-pneumonia, and of these fifteen per cent. succumb, and thirty-five per cent. recover. Immediate contact is not necessary for the transmission of the disease, and the first affected were among the furthest removed from the disease.

We believe that colonial ranchers were the first to inoculate with success, while the wise men of Europe were wrangling over minor points. It will be ultimately admitted that inoculation, like vaccination, is an accidental discovery, the credit of which may not justly be claimed by the leaders of pathological investigation. Professor Gerald Yeo has said :-

Care must be taken not to put aside too lightly long-established vulgar belief, simply on the score that it is not founded on a scientific basis or ly scientific observers. We must remember that vaccination, which certainly was useful when small-pox was rife, originated from common hearsay and vulgar belicf, and now is only supported by a few general principles which remain in the catcgory of pure empiricism. The pathologist finds it quite impossille to transmit the disease (plcuro-pneumonia) artificially by mediate communication, and the practitioner finds it impossible to control its rapid spread through a herd when introduced by a single 
diseased animal. In this respect it seems to stand alone among the diseases of the lower animals.

In 1885 Dr. Salmon reporteri to the American Government that inoculation did not lessen the losses, and was unable to stamp out the disease, and he suggested that it should be prohibited by law.

The recent Departmental Committec, after hearing all the evidence on the subject, report against the efficacy of preventive incculation, but they say: "We cannot deny that inoculation in itself is valuable as a palliative and preventive method of treatment.'

Rather a contradiction of terms to the unofficial mind, and suggestive of the hectored candidate who wishes to agree with the orthodox and compound with heterodox.

In Holland inoculation is said to have entirely succeeded; for whilst in $187 \mathrm{I}$, before inoculation was made compulsory, no fewer than 6,000 animals were slaughtered, only one was killed in $\mathrm{r} 886$, and again one only in 1887 . The act of slaughtering both diseased and in-contact animals has stamped the disease out of this country.

Lice.-At the end of the winter, colts, calves, and older stock are very apt to be crowded with these objectionable parasites ; they thrive best upon poor animals, finding a suitable home in the dirty matted hair in the late winter or early spring months, and on a sunny day may be seen literally in millions, every hair having nits upon it. One reason of so much rubbish accompanying them is that in the course of their development from the egg to the mature louse the skin is cast several times.

To get rid of them is not always easy, as the length of coat and accumulation of dandruff or scurf makes a waterproof covering which resists many certain destroyers if only brought into contact with the parasites.

A sunny day should be chosen, and the early part of it, when a bountiful washing with soft soap and hot water should be undertaken, so as to clear the skin of grease and dirt before applying the remedy. Stavesacre is an effectual destroyer of lice if prepared by boiling $\frac{1}{2} \mathrm{lb}$. with a gallon of water and brushing well into the coat with a hard brush. 
Tobacco juice is also much in request for the purpose, and can be procured wholesale at a very low rate, as it is imported with only a nominal duty, and the old expensire plan of boiling or infusing good shag tobacco is not necessary. By the way, very few people avail themselves of the governmental privilege of growing sufficient tobacco for this and fumigating purposes, though they might easily do so.

Paraffin is sometimes used, but is a very dangerous remedy, heing occasionally absorbed and causing the death of the animal, and not unfrequently causing a blister, and much un. necessary pain, and subsequent blemish.

There is another kind of louse from which horses suffer, which, if once seen, can never be forgotten - we refer to poultry lousiness. It will sometimes happen that a horse stabled with fowls will become affected and literally tear himself to pieces with them unless promptly treated with one of the foregoing remedies, either of which is as effectual against these as against the ordinary louse.

It is always well to repeat the dressing and keep the animals moving about till dry, or they may lick off more lotion than is good for them, or stand about and get clilled.

Some farmers get rid of lice among horned stock by completely covering them with linseed oil. This is really a very scientific way of slaughtering the parasites, as it will be remembered that their breathing apparatus is placed alongside the abdomen, and oily matter blocking up the respiratory apparatus causes their death. It certainly takes a good deal of oil to soak a beast all over, but it is not all lost, as he licks himself and his companions, and there is nothing more fattening and improving to condition. Many stock-owners give ol. lini regularly mixed with chaff and other food stuffs.

A good application, which a druggist might prepare, is liq. phenol, or Jeyes's Fluid, diluted to 1 in 40 , adding succus tabaci $\tilde{3}$ ij. to $\mathbf{I}$ gallon, or

Liq. Phenol Inf. Cuassize ad 
Inflammation of the Brain is occasionally met with as a result of blows or other external injuries, and may in some rare cases arise from tuberculous habit. Cerebro-spinal meningitis is the form taken. If the condition is correctly diagnosed, early bleeding from the jugular vein is recommended, two or three quarts being a perfectly safe quantity to take from a cow. At the same time a full aperient dose should be given, such as

$$
\begin{aligned}
& \text { Aloes Soc. . . . • • • zj. }
\end{aligned}
$$

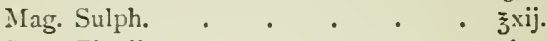

$$
\begin{aligned}
& \text { Pulv. Zingih, . . . . . うj. M. }
\end{aligned}
$$

Ice applied to the head by bandages of flannel attached to the horns, or to a head-stall if a polled beast. A low diet should be observed and gentle exercise as soon as practicable. If the condition arises from compression, and bleeding does not allay the delirium, it will be better to call in the butcher and not give medicine at all.

\section{Inflammation of the Kidneys. Symptoms.-Loss of} appetite, quick full pulse, very scanty urine, back arched, hind legs brought forward under the belly, pain in the neighbourhood of the kidney's, suppression of milk.

Treatment consists in the administration of demulcent fluids, as linseed tea, injection of warm water into the rectum at frequent intervals, a bran poultice over the loins, and

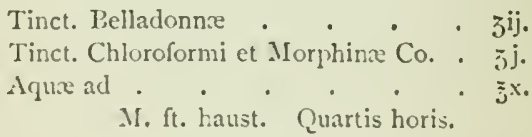

Cold.-The animal eats little, and the ears and legs are cold; is hide-bound and coat staring, soon followed by tears and mucous discharge from the nose. If the animal is benumbed, standing with its legs close together, give

Tr. Arnicx.

Sp. Camphore . .

l'ul. Capsici . . . . . jj.

With a pint or nore of hot becr. 
Cover the patient warmly for three hours, then $r . d$ down the whole body with a wisp of straw. Next day give a similar dose, and repeat the treatment on the third day. If purging ensues, discontinue the drink and give $\frac{1}{2} \mathrm{oz}$. of nitre dissolved in water. A little meal and honey may be mixed with the drink.

This is a suitable mixture for a cold :-

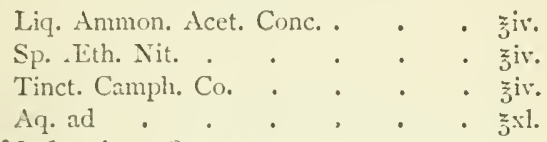

M. ft. mist. Quartam partem nocte maneque.

Cough results from cold, from dusty hay, or from so many causes that, without some history of the particular case under consideration, it is difficult to know what to prescribe ; moreover, in dairy cows coughs are quite common and do not affect the health or milk-giving qualities of the cows. Of late, however, a great deal more importance has been attached to chronic cough in cows, as it may indicate tuberculosis. Good clean food must be chosen and sprinkled with salt water. If cough is evidently accompanied with sore throat or tenderness on - pressure of the ribs, and there is reason to suppose it is of recent origin, the following is an old and tried friend worth consideration :--I part of black currant jam and 2 parts of honey in 18 parts of water, and give 1 pint of this night and morning. Mustard to the throat and sides if the cow turns with a groan, or a good smart liniment, such as lin. alb., or lin. camph. co. and lin. saponis, p. æeq. is also recommended. Mustard, if made thin and well rubbed in, answers well enough, but if a bullock has a thick coat the liniment will find its way through to the skin more readily than mustard indifferently applied.

Tuberculosis is a cattle disease of very great importance, as recent bacteriological researches lead to the supposition that it is communicable to human beings, and although it has not yet been absolutely demonstrated that tubercle 
in man has been conveyed by tuberculous milk, there are very grave reasons for suspecting it. Only an expert veterinary surgeon can decide if an animal is affected, and his fee for an examination of suspects is money well spent, as, apart from the possible danger to milk consumers, the disease is hereditary, ${ }^{1}$ and cows known to be tuberculous should not be again stocked, but made ready for the butcher. It does not appear that tuberculous meat, if well cooked, has any deleterious effect upon consumers.

Treatment is not hopeful, and there will doubtless com? a time when slaughter will be compulsory. Palliatives in the way of cordials and carminatives seem to do good for a time, and may enable a farmer to put flesh on an animal with localised tubercle, and get the carcase passed as fit for food, save those portions from which tubercles have been removed. There is no doubt that the top note has been reached by the alarmists, who have failed to prove the communicability of bovine tubercle to man, and, at the time of revising this work, there is a tendency on the part of the most advanced bacteriologists to climb down from a position where it was asserted that nearly all cases of human consumption arose from tuberculous milk or meat. It is thought that tuberculosis has greatly increased among cattle, but the statistics of human tuberculosis have meantime shown a very great decline. Because the tubercle bacillus is the same to all appearance in all animals, including man, it should not be too hastily assumed that it is transferable from the bovine to the biped. Nothing

1 The new school of pathologists deny the hereditary nature of tulserculosis and insist on its infectiousness, the bacillus, according to their views, being most often conveycd acrially, though capable of transmission by ingestion. The latter method is thought to account for tabes mesenterica and tubercular meningitis in infants. The apparent heredity that every onc has olserved in families of human as well as bovine animals is accounted for by an increased susceptibility to the bacillus, while others are nearly or quite immune. This is the theory of the great men, and it will last until another supersedes it. Meantime wc can each 'think what we like,' as the defendant lady promised the Court she would do, when convicted for slander. 
short of experiment upon man could prove it, and this has not been done. The old plan of inoculating condemned prisoners and 'giving them a run for their money' has been condemned by all civilised nations, and so we must remain without accurate knowledge on this most important subject.

Tuberculin Testing.- This has assumed very great importance, as now being almost entirely reliable. It is practised by the injection of the products of tubercle bacilli into the blood stream of living suspects. A gradual increase of temperature, with a more or less characteristic swelling over the region punctured by the subcutaneous syringe, takes place in those cattle affected by tuberculosis. 'Thermometric observations are made every three hours after the ninth, and if the rise is continuous, and amounting to about 3.5 to 5 degrees, it is pretty safe to assume that the patient is tuberculous.

The operator should be expert in temperatures and familiar with cattle in health, or his observations are liable to be vitiated by overlooking such factors as previous excitement by travel, or dogs, or parturition. Animals should not be tested under such conditions, and it is one of the grievances of the British exporter of pedigree stock, that these valuable creatures have to be tested immediately on their arrival at a foreign port, when excited or ill with a sea voyage.

Milk-Blue.-This is indicated by blue spots in the cream. Give a generous diet and add a dessert-spoonful of powdered caraway in water daily till the blueness disappears. The milk-pails must be kept very clean and bright, or a fungus will appear on them.

Milk-Bloody.-Bloody milk is a condition often accompanying red-water and may be caused either by congestion or by injury to the blood-vessels of the teats through stretching them too much while milking. Keep only the milk from the sound teats, and let the milk from the others fall to the ground. If the teats swell, milk them dry, even if matter should come with the milk, 
Suppression of Milk often accompanies indigestion. It should ensure a full examination and investigation, as it may arise from many serious as well as temporary and trifling causes. It is always of importance from a money point of view, and a falling off of milk which lasts more than a few days is seldom overtaken. Cow-keepers should be advised to keep the cordial drenches recommended on pp. I63-4.

Tapeworm.-The animal falls away in condition, the coat is rough and staring; hide-bound and loss of flesh also indicate the presence of tapeworm. Take valerian, male-fern root, worm seed, garlic, of each 2 oz., powder, and give daily for I 2 days $\frac{3}{4}$ oz. to an ox, $\frac{1}{2}$ oz. to a cow, and $\frac{1}{4}$ oz. to a calf.

Thrush in Calves shows itself in whitish sores on the tongue or gums of sucking calves, and the patient refuses to suckle. Take $\frac{1}{2}$ pint of vinegar, a spoonful of honey, and a little alum; mix them together, and wash out the mouth with this thrice daily. For internal medicine, take $\frac{1}{2}$ drachm of rhubarb, r drachm of magnesia; give this in water twice daily, and continue the mouth-wash. This is an aphthous disease similar to the thrush of infants, and may be treated with mel boracis or a lotion of boracic acid and glycerine. 


\section{DISEASES OF SHEEP}

SheEp may be treated like cattle, reducing the cose to onefourth.

Red eyelids are always a sign of health; when they are white or blackish, the animal is ill.

The specific diseases of sheep are comparatively few, but they are peculiarly subject to contagious disorders which, owing to their gregarious instincts, are very difficult to check or cure. Moreover, where the flocks are at all extensive, it is almost out of the question to expect that attention to be given to each individual which is devoted to horses and cattle. The shepherd naturally tries to cure a flock by some general treatment, of salt, or ferri sulph. in the food, dipping or smearing for scab or ticks, lime for the feet, \&c.

Blood Diseases. - Sheep are liable to two contrary blood dangers, partly due to constitutional tendency, and partly the effect of unsuitable diet. In fattening sheep there is a risk of 'plethora,' from which condition apoplexy, anthrax, and liver diseases may ensue, while pasture on bleak lands, food soaked in moisture, excessive suckling of ewes, and shearing are all circumstances which are likely to bring about an anæmic and sometimes a dropsical condition. Plethora is manifested by symptoms simulating apoplexy. The animal separates itself from the herd, looks dazed and giddy.

The symptoms of anæmia are debility, coldness, and pallor of the visible membranes, and sometimes a dropsical condition under the jaw, described by shepherds as 'poke' or 'chocker.' A variation of diet in each case is obviously the most essential form of treatment. Plethoric sheep should hare salt taken from 
them and Glauber's salts mixed in their food instead. Anæmic flocks should be removed to low-lying, well-protected pastures; salt should be given and ferri sulph., and the food should be as dry as possible.

Anthrax is not uncommonly met with in sheep, and is, of course, a blood disease due to a specific bacillus. It has been confused with apoplexy and is sometimes called plethora, or a plethoric condition is spoken of as a predisposing cause. Anthrax usually affects the head or neck and fore-quarters of sheep, but it is analogous with quarter-ill in cattle, and in both cases is due to a specific bacillus. Vast numbers of sheep are annually inoculated on the Continent by the disciples of Pasteur, and they claim to have saved many millions of sheep in different districts of Europe. The British Islands have at no time been so subject to anthrax as France and the southern half of Europe. It is believed by our most advanced veterinarians that the bacillus might be finally destroyed if all affected carcases were completely incinerated. Burial, no matter how deep, with or without lime, as prescribed by law, fails to destroy the spores which are conveyed by earth-worms to great distances and for an almost unlimited time.

Grub in the Head (Bots).-In May, June, and July, the sheep is subject to the attack of a sort of gadfly, known as the sheep-bot (Estrus oris). The fly is twice the size of the common house-fly, and may be seen on walls or fences in the neighbourhood of flocks. It is a dull brownish fly, with large yellow head, two greenish cyes, and wings almost enveloping the body. The female instinctively secks to deposit its ova on the margin of the nostril of the sheep, and the attack of the fly for that purpose causes agitation and even terror in the animal. Flocks will pack together, all with their heads down towards the ground, while the outer ones thrust their heads between their forelegs. If actually attacked, they throw themselves on the ground or run from the flock at a gallop. The eggs are hatched in a few days, and the young larve crawl into the nostrils and sinuses by 
means of hooks, irritating the membrane, causing a glecty fow of bloody mucus, and occasioning a peculiar twisting of the head as if in pain, and often an unaccountable loss of condition. Sneezing, a choking sort of cough, loss of appetite, diarrhœea, and sometimes death, are consequences of the attack of this parasite. No treatment has been found more effectual than holding the sheep's nose over a vessel containing a mixture of tar and sulphur, burning, so that the fumes shall be inhaled. The attack of the fly may be to some extent prevented by smearing the sheep's nostrils with tar, fish oil, or other non-poisonous fly dressing.

Husk.--A worm generally described as Strongylus filaria is often found in the bronchial tubes of lambs and sheep, causing a form of bronchitis, similar to husk or hoose in calves (see p. r $8_{4}$ ), and a similar or the same worm is also likely to infest the alimentary canal, occasioning diarrhœea and dysentery. These parasites may encyst themselves in the lungs, causing a false tuberculosis. These parasites are probably taken into the stomach from pastures where they have been left by previous generations. How they find their way to the lungs has been the subject of much learned discussion. The parasite often does not cause much inconvenience to the adult animal, but in lambs it may occasion a most irritating, constant coughing, an asthmatic kind of breathing, pallor of the tongue, anæmia, wasting, dysentery, and death. Where the disease has broken out, the lambs actually affected should be brought into sheds or straw yards, and those not yet attacked should be taken to new dry pastures. One to $2 \mathrm{oz}$. of salt, and 6 to $8 \mathrm{oz}$. of lime-water may be given to each lamb daily. The following draught may be administered every three days :-

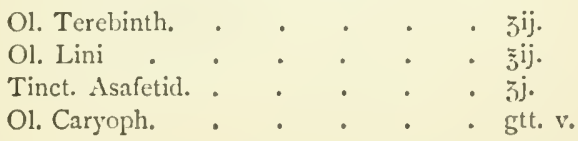

in linseed gruel or beer. Fumigation with tar, sulphur, or tobacco is a useful adjunct, and the daily injection of about 
Io minims of chloroform into the trachea is often of great service.

Husk is often treated by veterinarians of the modern school by intratracheal injections of terebene and hyd. bichlor., while mineral tonics, as ferri sulph. in 5 or 10 grain doses in combination with common salt, are administered; a little pulv. fœenug. and gentian make the medicament more palatable-an important consideration if a large flock is to be treated by giving it in the food and not as drenches.

Thrush, known as Aphtha simplex and Stomatitis, is a slight ulcerous eruption on the tongue, often affecting lambs, and sometimes, but rarely, attacking sheep. The saliva, which is bloot-stained, trickles from the mouth, and there is generally more or less fever and loss of appetite. Examination of the tongue shows crops of vesicles, which die away in a few days. The administration of Epsom salts and afterwards of pot. nit., by admixture with the food, is generally all that is necessary. There is another disease, called Aphtha maligna, which may at first be confounded with this, but which is much more serious. In this the eruption occurs on the lips, nostrils, and on the membrane opposite the gums. It is derived from a scabby sore which has broken out on the udders of the ewe, and is accompanied with fever in both the adult and the young animal. The same treatment as already indicated for thrush may be adopted, but the lambs must be taken from the dams and fed artificially. Lime-water may be given if diarrhcea be present. The sores on the ewes should be touched with nitrate of silver and then treated with a solution of alum.

Lambing.-The same bad results of parturition attend the flockmaster as the dairyman, and two or three per cent. of deaths among ewes is looked for at lambing time, even among the most experienced shepherds. Many chemists have found shepherds among the most painfully cunning of their customers, not excepting carters and grooms; but shepherds as accoucheurs deserve much praise, and many of them, when not possessing too large a hand, can deliver a cwe of twins far better than a 
fledgling from the R.C.V.S., 'bearing his blushing honours thick upon him.' It is, as a rule, only when a number of deaths follow rapidily that the shepherd seeks assistance from the reterinary practitioner.

In ewes, as in cows, the womb sometimes comes out, and has to be replaced and retained by stitches or an instrument. When accomplished, the animal should be kept short of bulky food, and the bowels kept soft by oleaginous aperients and bran slops.

Ewcs will often lamb well, and their progeny will frolic about, in dry hard frost, and no casualties occur from castrating at two and three weeks old; but in wet cold weather it is generally better to postpone castration, even if the lambs are rather bigger than they should be. For straining in ewes it has been the custom for generations to supply oleum viride, but a great improvement on this is I drachm of carbolic acid to $6 \mathrm{oz}$. of oil, whether 'viride' or ' rubrum.' An oil coloured with alkanet, and made odorous with a few drops of origanum, may be made a profitable, and, what is more, a most effectual proprietary article, as the antiseptic properties of carbolic acid are in this form best obtained, and a much larger percentage of ewes are sared where it is used.

\section{Scab in Sheep.-This} disease, which is of a contagious nature, is due to the presence of minute insects called 'acari.' The cure, therefore, depends upon killing these parasites.

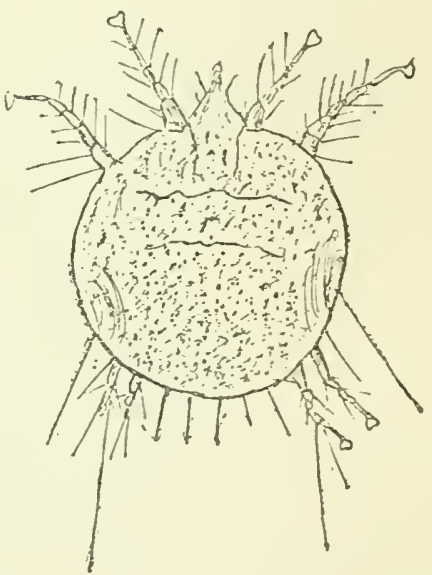

The Sheep-scab (Acarus).

An acarus is about the size of a pin-point, and when examined under the microscope is found to have an oval- 
shaped body, and four pairs of legs. Its presence on a sheep is proof positive of the existence of scab. Detection of the parasites is arrived at by gently scraping a little scurf off an affected part, and examining it under a microscope with a half-inch power.

The acari, on reaching the skin of a sheep, burrow into the skin, and there bury themselves for a time. Here the females produce their eggs, and in about sixteen days reappear with their litters of about a dozen young ones. These young ones again burrow, multiply, and reappear; so that in a short time an animal becomes infested with myriads.

The symptoms of scab are in accordance with the movements of the acari. When the insects enter the skin, minute red spots are left. Shortly, little pimples appear, which change in colour and size till a pustule is formed, and this bursts at the time the young brood is ready to appear on the surface. Of course intolerable itching accompanies all this, and the rubbing and scratching of the animal only aggravates the pustules, destroys the wool, and makes sores, which, drying, form the scabs from which the name is derived.

It is important to remember that acari may exist for some weeks on loose portions of wool, or on hurdles and trees against which a sheep has rubbed itself, and may then induce disease in any healthy animal coming in contact with them. In treating the discase it is not only necessary to kill the parasites on the sheep, but also to give a second dressing about sixteen days after the first, for the benefit of those which were beneath the skin on the first occasion.

Numerous substances are capable of destroying acari, and some of them may also destroy the sheep. Mercurial ointment is used very largely for smearing sheep, and washes containing corrosive sublimate have also been used. Both preparations are dangerous, and should not be employed. Arsenic is very largely used as a sheep dip, but it, too, is objectionable as poisonous. Not that there is any chance of a sheep being poisoned by absorption of poison through the skin. It is likely that no substance in watery solution can be absorbed by 
a. solind skin. Ointments, however, applied by rubbing, as mercurial ointment, are most undoubtedly dangerous. 'The following is a good arsenical bath for fifty sheep :-

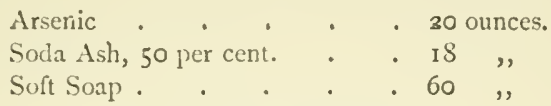

Add to this five gallons of hot water, and then forty-five gallons of cold. In this dip, the proportions are such as to leave a small excess of alkali. Among non-poisonous dips, sulphur is undoubtedly a valuable parasiticide, and may be used simply as an cintment, or in combination. Tobacco is a very efficacious and convenient application. It must not, however, be boiled, as the heat drives off valuable volatile principles. It must be prepared by infusion. The following form will not disappoint :-

$$
\begin{aligned}
& \text { Tobacco . . . . . I lb, } \\
& \text { Sulphur . . . . . . I lb. } \\
& \text { Size . . . . . . I lb. } \\
& \text { Water . . . . . } 5 \text { gallons. }
\end{aligned}
$$

The sulphur, of course, is merely suspended, and will require frequent stirring when in use. The object of the size is to make the dip slightly sticky, and thus allow the fleece the better to retain some of the sulphur.

Prevention should be carried out by keeping healthy sheep away from diseased ones, and from all places on which acari may be left, as raikwy trucks, hurdles, \&e.

Instead of treating the scab by one application, some authorities advise the use of a preliminary dip of alkaline water to soften the scabs, or of oil or glycerine well rubbed in for the same purpose. This is to be followed in two or three days by a poisonous dip. Nearly all advise that the scabs be rubbed with a stiff brush while the sheep is being dipped.

The quantity of dip required for each sheep is variously estimated at from one quart to one gallon. For small numbers of sheep, say 50 to 100 , the larger amount is necessary; while for large flocks, ore quart for shorn, or two quarts for unshorn sheep may be allowed. The dip shot:ld be kept while in use at 
a temperature of from $100^{\circ}$ to $110^{\circ} \mathrm{F}$. The addition of tar to dips serves a good purpose, as it is not only healing, but drives away flies.

The following are formulæ for some popular dips used for scab :-

\section{Texas Tobacco Dip.}

Tobacco . . . . 30 lbs.

Sulphur . . . . 7 llbs.

Concentrated Lye . . . 3 lbs.

Water . . . . . I00 gals.

Stcep the tobacco in three successive portions of water, expressing each time; then add the other ingredients to the liquor, and stir well while in use.

\section{Law's Sheep-dip.}

Tobacco . . . . . 16 lhs.

Oil of Tar. . . . . 3 pts.

Soda Ash . . . . . 20 lbs.

Soft Soap. . . . . 4 lbs.

Water . . . 50 gals.

Steep the tobacco as in the previous formula, and add the other ingredients to the liquor.

\section{Zundel's Carbolic Dif.}

Crude Carbolic Acid . • • • 3 lbs.

Caustic Lime . . • . . 2 llss.

I'otash . . . . . 6 llbs.

Soft Soap. . . . . . 6 llss.

Water . . . . . 70 gals.

Mix and boil.

$$
\text { Dr. Kaiser's Carbolic Dif. }
$$

Tobacco • . . . . $13 \frac{1}{2}$ llhs.

Soda . . . . . Sllhs.

Freshly slaked Lime. . . . 4 llss.

Soft Scap. . . . . 8 lbs.

Crude Carbolic Aciel (50 per cent.) . 4 llbs.

Water . . . . . 66 gals. 
Infuse the tobacco in the water, strain, and to the infusion add the remaining ingredients.

The following formulæ are from a work on the 'Animal Parasites of Sheep,' by Dr. Cooper Curtice, issued by the United States Agricultural Department.

\section{Carbolic Acid Dip.}

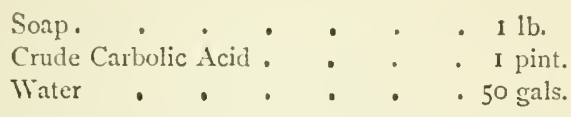

Dissolve the soap in a gallon or more of boiling water, add the acid, and stir thoroughly.

Keep the mixture well thinned, and do not let it get into the mouth, nostrils, or eyes of the sheep. Hold each sheep in the bath not less than half a minute.

\section{Eerosene Emulsion Dip. \\ Fresh-skimmed Milk. . . . 1 gal. \\ Kerosene . . . . . 2 gals.}

Churn together till emulsified, or mix and put into the mixture a force-pump, and direct the stream from the pump back into the mixture. The emulsification will take place more rapidly if the milk be added while boiling hot. Use I gallon of this emulsion to each 10 gallons of water required.

\section{Kerosene Soap Dip.}

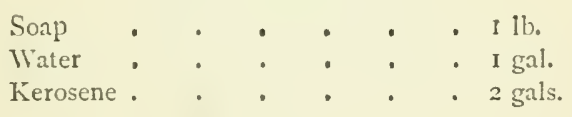

Bring the water to a boil and dissolve the soap in it ; then add the kerosene, and churn until emulsified. Use I gallon of this emulsion to 8 of water.

The above are rather prophylactic in their character, and are used generally after shearing. 
Other Parasites.-Besides the acari, sheep are very liable to the attacks of so-called ticks (Melophagus ovinus); also known as keds and fags, sheep lice (Trichodectes spharocephalus) and 'the fly' (Musca vomitoria). The tick is a dark red insect, with a white belly, with six clawed legs, and is sometimes nearly as large as a bean. Ticks are generally found on the neck and shoulders of ewes in spring, with their heads partly buried in the skin. They are very irritating, and if they pass, as they will, to the lambs, they 'often cause so much irritation as to seriously check their growth. If they are found on the lambs these must be dipped to get rid of them, but it is better to remove them from the ewes, either by dipping before shearing or by nipping them in two with the thumb and finger nails one by one, and lightly touching the skin around with a little mercurial ointment or turpentine.

Lice more often infest the inner part of the thighs and arms and the sides of the neck. Sheep will be noticed to bite at their flanks, to rub themselves and break the wool, and to scratch their elbows with the hind feet. Mercurial ointment is frequently used to kill the lice, and it does so effectually, but it is dangerous to apply it, especially in cold and wet weather. Tobacco-water with hellebore, or sulphurated oil, is almost if not quite as effectual, and is safer.

Fly or Fly-Struck. - In the early summer the 'fly' is a great nuisance to sheep. It lays its eggs on the wool, and as the maggots hatch they burrow into the skin of the animal and cause great irritation and sore places. The attacks of fly can be prevented by sprinkling a few drops of fish-oil on the sheep's wool carly in May, and fish-oil applied freely after the animal has been attacked will get rid of the pest, but will also reduce the value of the wool by giving it an unpleasint smell which cannot afterwards be got rid of. "Shepherds generally apply "stone mercury' (corrosive sublimate) to the place where the fly has struck, and this is effectual but dangerous. Spirits of tar applicd freely, and cutting away the wool in the neighbourhood of the spots attacked, will destroy the maggots and keep 
away the fly. The following is a common but dangerous application :-

\begin{tabular}{|c|c|c|c|c|}
\hline Tobacco Water & & - & & \\
\hline Arsenic & . & . & . & \\
\hline Soft Soap . & . & . & . & \\
\hline Asafetida . & . & . & . & \\
\hline White Lead & . & . & & \\
\hline Pearlash . & . & . & . & \\
\hline Sulphur & . & . & . & \\
\hline $\begin{array}{c}\text { Cinnabar } \\
\text { गix. }\end{array}$ & - & - & - & \\
\hline
\end{tabular}

The following is a good powder for preventing the fly striking, and has been in use for several generations where fly is troublesome :-

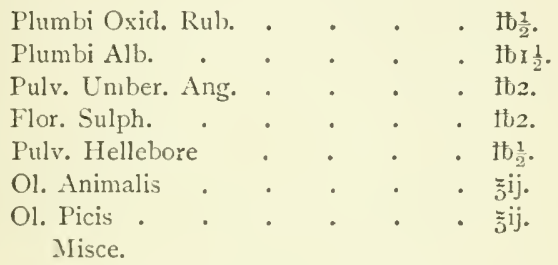

The oils are rubbed down with a small quantity of flor. sulph. at first and more added until the powder in bulk is not damp, but should be passed through a sieve before sending out in I lb. packets labelled as follows :--

\section{FLY POWDER FOR SHEEP. POISON.}

Directions for Use.-It should be applied when the dew is on the sheep, or, otherwise moisten the fleece with a garden water-pot and rose. Part the wool down the back and elsewhere if necessary and apply the powder by means of a flour dredger. The hand should be held over the sheep's eyes while the head is well sprinkled, as fly will strike where any sores are caused by fighting.

This packet is enough for TWEXTY SHEEP. 
Rot in Sheep (Flukes).-To the non-agricultural mind this expression is not in the least explanatory, and the amateur may confuse it with another disease affecting the feet. Rot, so called, is a very serious disease, arising from the presence of parasites in the liver of the sheep. They are called flukes, and occupy the bile-ducts to such an extent as to block the current of bile both by their bodily presence and their excrementitious matter, which is of a granular nature, especially calculated to fill up these small but important channels. Small numbers of these undesirable guests may be entertained without any serious inconvenience to the 'host,' and rery few Welsh sheep are found to be entirely free from them when slaughtered.

In wet seasons and upon low ground (always excepting salt marshes) rot may be most dreaded, the reason being that flukes pass through several stages of development in the small molluscs that can themselves only subsist in moist places. It is only in exceptional seasons that flukes are of any account on the South Downs or the chalk hills of Surrey, but the Fens are never free from them. When they occupy the liver in large numbers their presence interferes with the portal circulation, and dropsy is the result. During the first six weeks when sheep are attacked with fluke or the rot, the animals will decidedly improve in flesh by the stimulation of the liver, and wise flockmasters take advantage of this to kill them before the muscular fibres become flabby, or, in other words, the flesh becomes unfit for food. If allowed to go on, the sheep rapidly loses flesh, becomes 'razor-backed' and 'pot-bellied,' like a rabbit fed entirely on green-meat, the wool comes out in handfuls, and the wretched creature wastes to a skeleton.

'To kill a parasite in such a sccure retreat is obviously impossible, as he cannot be got at through the stomach, and any remedy intended to act through the circulation must be strong cnough to kill the sheep. In making this remark we are aware that many persons with more zeal than anatomical knowledge believe they can kill fukes by the administration of ol. turebinth., sodii chlorid., chickweed tea, nettle tia, and other remedies, but they are deceived by the fact that 
sheep improve in the early stage without remedies at all, and fatten more rapidly if given cordials, but only during that period.

There is good reason to suppose that sheep having but a few flukes practically recover when turned on salt marshes : sufficiently, that is to say, to be made moderately fat and saleable for food, but the 'cure' is not lasting, and a flock of ewes would be no use saved for another year when once really infected.

Sheep-pox.-Sheep are subject to an cruptive disease having just the same characters as cow-pox, and, according to Professor Simonds (formerly Principal of the Royal Veterinary College), not communicable to the cow or to children. There is, too, in sheep a malignant form of pox in which resicles are not produced, the victims lose their wool in matted lumps, their eyes undergo rapid changes terminating in blindness, their nostrils are stopped up with a horrible matter, and their skins crack like clay in a hot sun. In scientific nomenclature sheep-pcx is divided into

(I) A malignant or confluent form ;

(2) A benign or discrete form.

The history of this disease is a little obscure, but it is thought to have existed in England many centuries ago, and to have disappeared, to be reintroduced in $\mathrm{I} 8+7$ by some merino sheep brought from Denmark. The disease was traced to Russia, where cattle plague and pleuro-pneumonia are commonly believed to be permanently located. Professor Simonds was sent out by our Government to investigate this 'Eastern question,' and, in his lectures to the students of the R. V.C., used to say that we might look forward with reasonable certainty to cattle-plague travelling westwards again, whenever a great European war should render the morement of great herds necessary, and the existing strict rules on the western frontiers become relaxed. The same remarks doubtless apply to sheep-pox if it be true that it has a permanent home in Suuthern Russia. 
Giddiness is rarely curable, and as the flesh is harmless it is best to kill the animal at once.

Garget.-Inflammation of the udder is more frequent with the ewe than with the cow. When it is so painful that the mother refuses the lamb, the udder should be carefully fomented with warm water, and the following ointment may be applied :-

$\begin{array}{lllllll}\text { Camphor. } & \cdot & \cdot & \cdot & \cdot & \cdot & \mathbf{j} \\ \text { Ung. Hydrarg. } & \cdot & \cdot & \cdot & \cdot & \cdot & \mathbf{j} \\ \text { Ung. Viridis. } & \cdot & \cdot & \cdot & \cdot & \cdot & \bar{j} \text { j. }\end{array}$

In cases where the udder continues to swell, incision must be had recourse to, and a lotion of chloride of lime ( $5 \mathrm{ij}$. to $\overline{\mathbf{j}} \mathrm{vj}$.) applied. When the putrid smell has gone, the wound may be healed with tinct. benzoin. co. Lin. camph. is an excellent application. The lamb must not suck an udder so treated.

Swollen Udder.-Rub together the white of an egg, some saffron, and olive oil, and rub the udder with this thrice daily. Milk the animal so long as the disease lasts, and give internally twice daily, to remove the hardened milk, I drachm of a mixture of potassium sulphate 4 parts and nitre I part. Give once I drachm of nitre and 2 drachms of common salt dissolved in water.

Consumption or Tuberculosis is not so frequent a disease among sheep as it is with cattle. The greater time spent in the open air is thought to account for the comparative immunity of sheep, but there is probably some other factor, of which we are not yet able to take account.

'The symptoms resemble those of rot-cough, falling of the wool, and paleness, swelling of the eyes, \&c. Mix juniper berries, roasted acorns, and gentian, of each $\frac{1}{2} \mathrm{oz}$., add $\frac{1}{4} \mathrm{oz}$. common salt, and give an eighth part night and morning.

Cough results from cold. Mix powdered fennel, elecam1 anc, and flowers of sulphur, of each 2 oz., and give two tea- 
spoonfuls twice daily in food. If the cough results from dusty food, see that the cause is removed.

Diarrhœa is often the result of sour pasture. It is also parasitic. Mix $\frac{1}{2}$ oz. juniper berries and as much chalk, and give a teaspoonful several times a day. If blood is present in the excreta give often I drachm each of rhubarb and magnesia and $\frac{1}{2} \mathrm{oz}$. of honey. Some practitioners place great faith in $5 \mathrm{j}$. doses of alum combined with chalk and gentian. For sucking lambs put a piece of chalk in the stall for them to lick, and give in ewe's milk I drachm of magnesia twice daily. For bad cases boil I oz. gentian root in I pint of water, strain, and mix with the decoction I drachm of opium. Give a teaspoonful every two hours. Calamus root does good service.

Gid, Sturdy, Turnsick.-The condition known under these names is occasioned by a species of hydatid (Conurus cerebralis). This is the median stage of a tapeworm of the dog (Tenia Conurus), which, in order to complete its development, uses the sheep as its intermediary host. It has the appearance of a small bladder filled with pellucid water, and establishes itself in the brain or between its two hemispheres. As it grows it presses on the brain and causes the sheep to hold its head always on one side, and as it grazes to always rotate towards that side. The brain is affected, and the animal is alway's frightened at any movement. There is no effectual remedy, and the sheep should be killed. In view of the danger to dogs, the excrements of the diseased sheep should be destroyed. None of the heads of sheep containing the cyst should be given to the dog.

Hoven in sheep is of similar origin to the same disease in cattle and may be treated in a similar manner, but with proportionally reduced doses.

Red-water, or the effusion of a bloody fluid in the abdominal cavity (sanguineous ascites), is a frequent and often fatal disease among sheep. It is generally the result of the 
removal to a cold damp pasture, and happens when hoar frost occurs. The administration of sulphur as an aperient in doses of I oz. daily ( $\frac{1}{2} \mathrm{Oz}$. for lambs), salt in the food, and a complete change of diet and surroundings, are essentials of the treatment.

Foot-rot is one of the most frequent diseases which the druggist has to treat. It may be occasioned by damp and dirty pastures, or by dry, sandy, and gritty ground. Foot-rot is very common among sheep, especially on some soils, and indeed is never absent from some flocks in the wet seasons. It does not appear to be infectious as between one sheep and another, though it should be stated here that professional opinion is divided on this point, and exhaustive experiments have been carried out with a view to elucidate the point.

It would seem that land carries it, and that future pasturers will get the disease from land on which subjects of foot-rot have been fed, although actual transmission from the presence of foot-rot matter may fail to produce the disease when inserted into the scraped foot of a sound animal.

The symptoms are lameness and local inflammation; the hoof separates from the coronet, and large ulcerating sores appear, and in these cases much harm may be done by the unsparing use of the shepherd's knife. 'The fore-feet are generally the first attacked, and the animal affected will in this case graze on its knees, and lie about. The foot is hot and tender, the coronet swollen, the horn becomes soft and apparently rotten, pieces of it becoming detached. Where the sensitive structures of the foot become exposed, fungous growths occur, which ulcerate, bleed, and discharge a thin very foul-smelling matter. 'The disease is most prominent in the early autumn, and particularly after a wet summer. 'The treatment consists first in carcfully cleaning the hoofs, and with the proper instruments paring away all loose and detached horn. Moisten loam with rinegar, put it in a bag, stick the foot into the mass and tie it up. Repeat this several times daily, cleaning out the matter from the elge of the eleft in the hoof, and cutting away all 
diseased horn. Nake a solution of alum $\frac{1}{2}$ oz., green vitriol $\frac{1}{2}$ oz., in $\frac{1}{2}$ pint of water; dip tow in this and place it in the wound, and cover it with dry tow. When it is thought necessary to give special care to valuable animals, poulticing is adopted. Daily dressings of butter of antimony, or preferably butter of antimony and tincture of myrrh (equal parts), are frequent methods of treatment, and many other applications have been recommended. Several formulie for foot-rot dressings will be found on page 3 I 7 . 


\section{DISEASES OF PIGS}

Swine Fever.- The diagnosis of the disease is not always easy, owing to the modifications it has undergone during the past few years; so difficult is it that the Board of Agriculture do not even now rely upon the diagnosis of an M.R.C.V.S., but require the viscera of the suspected animal to be sent to London, where their experts examine, make cultures, and only decide after the specific bacillus has been found. Treatment should not be attempted, as the disease comes under the Contagious Diseases (Animals) Act, but owners of pigs should be advised to give notice to the police authorities immediately, as no compensation is allowed for pigs that are found dead, and a few hours may make a difference of many pounds. The owner must give notice that he believes the animals to be affected with swine fever, or the police will not act. Many cases have occurred in which much injustice has been done to owners; the police asking for a certificate from a qualified $\mathrm{V}$.S. before undertaking to send their own V.S., who may or may not be qualified, according to the date of his appointment. ${ }^{1}$ The time lost in obtaining the necessary certificate often results in the official visit being made when the majority of the infected pigs are dead.

Parturient Fever is sometimes met with in sows, and the same treatment as in the case of cows may be adopted; but it is very difficult to treat sows, as they are proverbially obstinate, and drenching them is a serious business. Many pigs will cat food in which mag. sulph. has been dissolved, and

1 No unqualified men are now appointed. 
flor. sulph. added to wash is not usually rejected. When it becomes necessary to give a nauseous drench, there is no better plan than the old method of cutting off the toe of a stout boot and introducing that end of it into the sow's mouth while pouring the drench in at the other end.

Protruded Rectum.-In sows the rectum is sometimes pushed out during parturition, and after. Young pigs, and in fact swine at any age, are liable to this very unsightly and painful condition, particularly in cold weather. It is very difficult to control a sow and replace the rectum, as she will oppose all her strength to her benefactor, and when sutures are put in they seldom last, or else the rectum has to be unloaded by the hand of the attendant. In young pigs the operation is fairly successful if the diet is looked after. If, instead of blowing them out like drums with sloppy food, they be kept empty except for a little corn to 'stay their stomachs' for a few days, all will be well with them, and the old diet may be gradually resumed; but with large hogs it is better to use a powerful astringent and let the rectum shorten itself partly by shrinking into its proper position, and partly by strangulation and sloughing of the outer portion. Of course, it is best, if possible, to return and retain it when first done, but the owner does not usually consult a practitioner for a day or two. The following ointment can be applied when, from the size or ferocity of the sow, niecharical assistance cannot be given :-

$$
\begin{aligned}
& \text { Alum. Exsicc. . . . . . } \boldsymbol{\zeta}_{\mathrm{j}} \text {. } \\
& \text { Adipis . . . . . . } \xi^{3}
\end{aligned}
$$

Some practitioners facilitate the sloughing process by painting a line of acid: carbolic. fort. at about an inch from the buttock, but it is hardly a safe plan to recommend, and owners are ever ready to advertise an insuccessful remedy.

Rheumatism in Pigs frequently upsets the calculations of the pig-breeder, by making cripples of young and thriving pigs quite suddenly and without any apparent cause. Of 
course there have been theorists to suggest wet and ill-drained styes \&c. as a cause, but who ever knew pigs to be kept dry? 1 Our own experience is that they are just as liable to rheumatism, or joint ill or evil, as it is variously termed, whether they be kept in the well-drained and cleaned buildings of a model farm or in the filth of a cottager's stye, with no floor but the earth, and no bedding but garden refuse. The knees and fetlocks are the parts most often severely affected, much painful swelling and lameness resulting.

Whether it will be found that so-called rheumatism is due to a specific bacillus in erery case, it is impossible to say, but there is an increasing amount of testimony as to the presence of some deleterious microbe in many of the joint diseases of young animals. These have been more particularly noticed under the heading of Rheumatism in Cattle (see p. $\mathrm{I}_{3}$ ).

Outward applications often benefit pigs in the early stages of the complaint, and some recover completely ; but it is very little gocd to adopt any treatment when the enlarged joints have become hard and the lameness chronic; such pigs seldom thrive and are not worth keeping; they should be killed when the accompanying symptoms of fever have abated after a saline purgative or two. They are fit for human food if the temperature, as ascertained per rectum, is not over $100^{\circ}$ Fahr.

An excellent application is the following :-

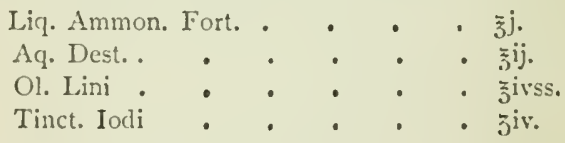

M. ft. linimentum, quotidic applicandum.

Or this :-

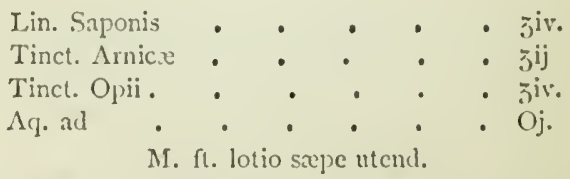

1 Injection of a 4 per cent. formalin solution near ll:e joint has becn recently tried with grent success. 
In the meal or wash may be given pot. nit. and sulph. nig. in doses of from five to ten grains of the former to one drachm of the latter daily, to pigs from two to six months of age.

This rheumatism in pigs is commonly known as joint ill, joint fellon, or lameness from swollen and painful joints. As already stated, it may affect pigs at any age, but it is particularly frequent among young pigs. It is to a large extent hereditary and avoidable; but it often happens that people buy a sow for breeding, and, if they inquire at all about her, it is as to her pedigree and not her hereditary diseases, these being quite as common among the aristocracy of the stye as among crossbred and less valuable animals.

This disease, pathologically, closely resembles rheumatic gout in the human subject, and it is more prevalent during the east winds of spring and after the autumn rains than at any other time. There is first stiffness and unwillingness to move, then swollen joints and constipation; great heat and tenderness often accompany this complaint, and a rigidity in some cases that resembles the effects of strychnia.

Treatment, as we have explained, should be undertaken in the early stages of the disease, to be effectual. Along with the application of the lotion ordered above, a brisk aperient of croton oil ( $m \frac{1}{2}$ to $m$ ij.) and castor oil ( 3 ij. to 3 iv.) should be first given, and afterwards salicylate of soda with colchicum and potash. The following is a suitable mixture :-

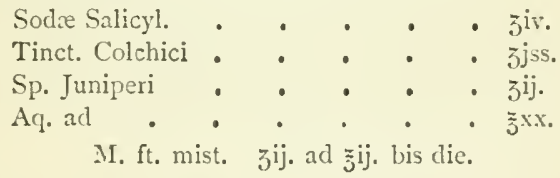

Or this :-

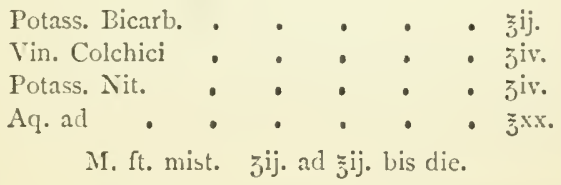

Doses of these mixtures may be administered alternatively 
with advantage when the inflammatory action has been reduced. An embrocation should be employed to excite absorption of the gouty deposit which usually results. The following lotion is suitable :-

$$
\begin{aligned}
& \text { Tinct. Iodi . . . . . . jiv. } \\
& \text { Liq. Potassæ . . . . . 3ij. }
\end{aligned}
$$

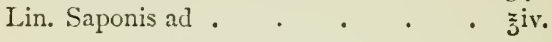

II. ft. enibrocatio.

The joints to be rubbed with this daily.

The draught recommended under Diseases of Cattle (Rheumatism, p. I 84 ) is suitable for pigs if the dose be reduced in proportion to weight.

Inflammation of the Lungs.-The prominent symptoms are loss of appetite, incessant and distressing cough, and heaving at the flanks. At the very first the animal must be bled, preferably from the palate. Give 2 to 4 drachms each of sulphur and Epsom salts, according to the strength of the animal. Follow with a powder of digitalis, 2 grains; antimonial powder, 6 grains ; nitre, 30 grains; twice daily. Keep the animals clean, warm, and well fed.

Loss of Tail. - High-bred pigs are very apt to lose their tails when young. The disease shows itself when a day and a half old. A red spot first appears, and gradually spreads all round the root of the tail, destroying the cuticle. If once round there is little chance of saving the tail ; but if at the first all the red place be well scraped with the nail or a penknife until it bleeds, and afterwards greased, a scab will form, a cicatrix follow, and the tail will be saved.

Cough. - Slight coughs are cured by feeding with sour milk. For cough resulting from chill, make an electuary of aniseed, $1 \frac{1}{2} \mathrm{Oz}$; ; hquorice, ${ }^{\circ} \mathrm{I} \frac{\mathrm{l}}{2} \mathrm{Oz}$. ; honey, $4 \mathrm{Oz}$.; a piece the size of a walnut to be mixed with a little sharps or other farorite food night and morning.

Diarrhœa resulting from cold. Give an ounce of juniper 
berries once, or $\frac{1}{4} \mathrm{oz}$. tormentilla root twice a day. Allow no milk. Diarrhoea, or scouring, in sucking pigs may be treated by giving the sow a few old beans. 'Calves' Cordial' is the best medicine for diarrhoa in pigs (see Formulæ).

Parasites are a frequent cause of diarrhoea in pigs of all ages. The evacuations should be carefully examined and suitable anthelmintics prescribed. For round worms santonine and ferri sulph., and for the tapeworm class areca nut. Half a grain of santonine to each pound weight of the pig, and two grains of areca nut, is a safe and, at the same time, fairly strong dose. These agents may be given in a very sma'l quantity of milk or sloppy food after fasting twenty hours. Pigs should never be forcibly drenched if they can be induced to take medicaments in any sort of food. Dropsy of the belly is in most cases due to poverty, and the subjects of it recover with improved diet. In a few instances it is due to some interference with the portal circulation, or the blocking up of the bile ducts with parasites or their excreta. Flukes, which cause the 'rot' in sheep, may affect pigs, and with much the same consequences. A dropsical state in young pigs arises often from the food being very sloppy-nearly all water in fact, and devoid of sufficient nutritive material for growth and development. In old sows it may be due to the frequent bearing of farrows of pigs and poor food while suckling them.

Treatment.-This is fairly hopeful, as the pig is a great glutton and possessed of such powers of assimilation that he can put on flesh and support a host of parasites as well, and the merely pendulous belly with a lot of unnecessary fluid in it may prove inconvenient, but so long as he will fatten he is worth keeping. Small doses of sulphate of iron, quinine, and a vernifuge (see Diarrhcea) will be found helpful. Table salt may be given in small doses, but large ones are apt to induce an eruption ('the red soldier') and bring piggy into the notice of the village constable as a suspicious characterthat functionary having always before his mind the detection of swine fever, and the kudos attaching to its discovery by a member of the force. 
Eye Diseases.-Pigs are not very much troubled with maladies in connection with the visual organs, and this is only consistent with the popular idea that they can 'see the wind.' Sccond sight of this kind should enable them to avoid a draughty corner in the stye. A glassy or mucous accumula. tion occasionally affects growing porkers, and this should be treated with a boracic lotion. The edges of the lower lids may be anointed with vaseline or any simple ointment to prevent accumulation of sticky matter.

Gripes or Colic.-Pigs are liable to colic as a result of unsuitable food. It is not difficult to diagnose, as they double themselves up and behave very much like human beings in this respect (as well as in others).

Treatment.-A dose of castor-oil with a few minims of ol. menthe pip. If relief is not thus obtained, a dose of 'Calves' Cordial' containing tinct chloroformi et morphinæ should be given (see p. 161).

Maggots in the Ear.-If a pig has a sore place anywhere that he cannot conveniently get at, flies are apt to blow it and produce maggots. The ear is one of those situations, and the tail is popularly supposed to be another, or we should never have had those interesting lines handed down to us respecting the farmer's dinner party, whereat

One talked of miliew, one of frost, and one of storms of hail, And one of pigs that he had lost with maggots in the tail.

One wiped his nose upon his slecve, one spat upon the floor, Ancl, not to give offence or griete, held up the cloth hefore.

Maggots occurring anywhere may be easily disposed of by a carbolised oil dressing, and customers should be advised to apply twice. One in twenty of ol. rubrum is a perfectly safe application.

Quinsy, Strangles, or Gloss-anthrax.-One of the 
most malignant of the discases of swine. A specific germ is the chief cause, and the disease is commonest in spring and autumn. There is a loss of appetite, the ears hang, the nostrils secrete mucus, the head is shaken, the mouth is dry and hot, the ears cold, the eyes watery, ultimately the throat swells, the tongue becomes red and then brown, the roice lower. Gire daily in whey I drachm saltpetre and $\frac{1}{4} \mathrm{oz}$. Glauber's salts. If the pig will not, or cannot, swallow, mix 4 oz. Glauber's salts and 2 oz. nitre with enough honey to make an electuary, and rub $\frac{1}{2}$ oz. on the tongue every four hours. Youatt thus describes the disease and treatment :-The glands under the throat begin to swell, respiration and swallowing are impeded, hoarseness and debility supervene; the neck swells and rapidly goes on to gangrene, the tongue hangs from the mouth and is corered with saliva. Bleeding and purgatives are indicated, with setons and punctures of the swollen glands.

It is often caused by soda in the 'wash' taken from town houses, the scullery maids using soda in dish washing, and pouring the greasy fluid into the hog tub. Indigestion and the presence of internal parasites have the effect also of producing a redundancy of scurf. A thriving pig has generally a softer skin than a poor one, and the saying of 'greasing the fat hog' has its origin in the stye. A show pig already fat will get his back anointed with linseed oil, while a starveling, that would derive still greater benefit, is forgotten or not deemed worthy the trouble. Itching may be merely an exanthematous condition due to the foregoing causes, or due to external parasites, as lice or mange. In the latter a greasy smear should be made and not an aqueous dressing, as we have seen recommended, with instructions to 'wash the pig frequently;' in forgetfulness of the fact that it will return like a sow that is washed to her wallowing in the mire. Either an ointment of sulphurated potash or sulphur in linseed or other thick oil should be used. If the rancid pomades and other greases have not been used up in making hoof ointments, they should be brought out when such a dressing as this is needed. 
Snuffies, Sniffles, Nasal Catarrh.-There is first a slight discharge of mucus from the nostrils, gradually increasing till it causes cough, sneezing, and difficulty of breathing. The membrane of the nose becomes thickened and the nostril swollen and deformed. Blood is often discharged from the nostril, which gives temporary relief, but the hemorrhage is apt to recur, and so undermine the animal's strength. The best treatment is the administration of copper sulphate night and morning, in doses of 3 to 5 grains, with good food and cleanliness. The disease is often fatal, and it is generally well established before it is noticed.

Sprains, Sores, and Bruises.-Collections of pus must be opened, washed, and anointed with turpentine oil till healed. Bad bruises, resulting from blows, should be rubbed with a misture of 2 oz. soap and I drachm powdered camphor. Sprains may be treated similarly.

Stye or Blaine.-A white blister the size of a pea on the tongue, which indicates a violent and very dangerous fever. There are also loss of appetite, dull eyes, rooting with the snout, trembling, and uneasy grunting. Relief is sometimes obtained by opening the blister and rubbing the wound with salt and vinegar.

Worms. - It does not always pay to keep pigs, and never does to keep worms. If any are noticed in the dung, measures should be taken at once to get rid of them, as they multiply with astounding rapidity. If a pig does not respond to the ration he is receiving, and shows no signs of illness, he may justly be suspected of worms, and suitable remedies prescribed. 'These are santonin, powdered glass, dolichos, buchu, salt, turpentine, areca nut, and ol. filicis maris. It is most difficult to lay down the dose for animals varying from two pounds to two hundredweight, but we have found a fairly practical working scale by estimating the pig's weight to that of the human child or adult, and giving proportional doses. 
Cod Liver Oil for Fattening Pigs.-In the 'Lancet,' November 5,1853 , I)r. Pollock published an account of some interesting experiments made by an Essex agriculturist regarding the fattening action of cod liver oil on pigs, shcep, and cattle. Twenty pigs separated from a lot of three hundrcd, averaging in weight from five to fiftcen stones, received two ounces of oil daily with as much meal as they pleased. The rest of the lot were treated in exactly the same manner, lout got no oil. Those receiving the oil are stated to have consumed less food, and when killed weighed the heaviest and made the most money in the London market, the fat being firm and white. When the daily allowance of oil was increased to four ounces per day the fat became yellow, and the flesh acquired a fishy taste. For small pigs an ounce daily was found the most economical quantity. It is not certain that the regular administration of other oil might not have been equally efficacious. 


\section{DISEASES OF DOGS}

THE ailments of dogs are various, and to prescribe successfully requires some familiarity with breeds, the relative weight of dogs as compared with the human body, their age as shown by the teeth, \&c. But the prescribing druggist will best consult his own as well as his client's interests by not attempting operations requiring an intimate knowledge of the dog's mechanism. The physiology or vital processes of human and canine beings are very similar, man and dog both being omnivorous.

Sickness. - Tomiting when observed in the dog may be of his own seeking, and in this sense remedial, inasmuch as the dyspeptic animal, when he can procure it, will eat a few blades of couch-grass (Triticum repens), and be soon relieved by romiting a frothy matter-composed of bile which has made its way through the pyloric end of the stomach, and of gastric juice which has been rapidly secreted by the mechanical irritation of the rough grass when swallowed. Sickness, again, may be induced by swallowing unmasticated food. Dogs are not, therefore, necessarily ill because they romit; and it may be assumed further that, with them, the scnse of nausea cannot be present, or they would not, as they do, in the language of Holy Writ, 'return like a dog to his romit.'

Sce also (iastritis and so-called Canine Influenza at p. 24.

The Clinical Thermometer can lest be used by passing it into the rectum for about one inch, and holding the tail at the same time. Hardly any dog will resent this liberty, and without this instrument it is impossible accurately to gauge the 
temperature, as the practitioner is misled by the coldness or otherwise of his hand, the weather, \&c. 'The popular notion that a cold nose is a sign of health is only partially true-a dog may be very ill, yet have a cool moist nose, or in perfect health, yet, lying in front of a fire, as dogs are so fond of doing, have a dry hot nose.

To Hold the Dog.-It may not be out of place to remark lere that among the initial difficulties of dog-doctoring is that of securing the patient in a position at once safe to the examiner and comfortable to the dog. He should not be held upon the lap of his mistress, as she will probably suggest, in order to calm his fears, for in that position he resents interference, and fancies he is guarding the sacred person of his owner. 'The same objection applies to seeing dogs at their own residences -if one may use the term-for here they are like the cock upon his own dunghill, and the dog that threatens you with big swears at home will often be as mild as the proverbial 'sucking dove' when brought to your shop.

In trying to examine any dog of doubtful temper, or who has been unfortunate enough to acquire the bad name which is so tenacious among his tribe, it is well to arm yourself with a long narrow strap, which should be placed on his face, crossed below his jaw, and buckled behind his ears.

With this contrivance you can defy the most sarage dog, and throw him upon his back to extract a decayed tooth or other minor operation. To keep a large dog down on the ground it is a good plan to attach three or four feet of rope to his collar and pass it twice round the hind leg that is uppermost, in the hollow just above the hock, pull it well forward, and attach it again to the collar. If an abscess is to be opened or a festered nail remored, this position will ensure safcty to the operator and all concerned. The only assistant who should be disqualified is the owner of the $\operatorname{dog}$, as his eflorts to soothe the unfortunate beast are apt to mislead the animal, and make him suppose he is being thus treated against his master's will. 
The Administration of Medicines must in a large measure depend upon the nature of the remedies prescribed, but where compatible with the prescriber's notions of the case he will always select powders. Pills have the great objection that the majority of people cannot drive them down, and liquids are more frequently distributed indiscriminately over the administrator and the patient than passed down the gullet of the latter.

Powders, on the other hand, when not extremely bulky, have only to be thrown into the mouth, or upon the tongue, and the saliva will give sufficient moisture to cause them to be swallowed, and insufficient to allow them to be dribbled out again. When, however, remedies are desired which cannot conreniently be made up into powders or electuaries, they should be made up, with some excipient, into very large pills, at least ten grains for a small dog, as it is much easier to give a large pill than a small one, and quite as safe, as it is to be borne in mind that the cesophagus or gullet of the dog is very large, so large that he can swallow a bone which he cannot afterwards pass through his bowels. The method of administering a pill is to take hold of the dog's face, with your left-hand palm pressing on his nasal bones, while your thumb and forefinger exert a gentle pressure on each side of the upper lip, immediately over those large teeth called canines in the human being and tusks or tushes in the dog. This gentle pressure will cause him to open his mouth, and not close it on your other hand, if by the pressure of your left you make the lips just overlap the teeth. The right hand is employed in giving the pill, which should be held between the tips of the first and second finger, and pushed with some degree of force right over the back of the tongue, regardless of all anatomical considerations, as if you were trying to push it through the back of his head. If it reaches the fauces he will be sure to swallow it, as the act from there downwards is involuntary ; there is no danger whatever of its going the wrong way, as the epiglottis, or valve-like structure over the windpipe, is set on guard over that aperture the moment an aggressor enters the mouth. In no case use a gag or spoil your ruler by placing it crossways in his mouth, as 
'force is no remedy' with dogs, whatever else it may apply to.

In cases where you want to give ethereal preparations, for which pharmacists have not yet provided us a capsule, it will be necessary to drench the animal. 'This, again, is not done by a gag, or by attempting to hold the mouth open ; on the contrary the teeth may remain shut, but a glance at them will show that plenty of room exists between them for a teaspoonful or two of fluid to make its way through the apertures. Having secured the dog, with or without a muzzle, insert your finger in the corner of his mouth, and pull out his lip, which will make an excellent funnel into which to pour his draught, and as soon as you relax your hold he will open his mouth and swallow it, to his own surprise as well as yours, if you have never tried it.

Distemper.-If a dog is ill he is commonly supposed to have the distemper or be mad, and the general public make but little distinction. Distemper is common to most dogs as an infantile disease ; rabies is very rare, and there are many experienced veterinary surgeons who have never seen a genuine case of the latter.

Distemper is a term which formerly implied any disease of a prevalent nature or epidemic to man or animals, but the meaning of the word has narrowed down in the present day to the exclusive use of dog fanciers and whitewashers; though why the washing of a wall should be 'dístempering' it, none of the faculty seem able to, explain. All the old works speak of animal plagues as 'distempers,' and the literature of the time of the Plague of London often adverts to the 'distemper now raging,' \&c.

It is as well to state that there is no specific for distemper, and the professional man who vends an infallible cure for it, elegantly prepared, is as great a humbug as the stable loafer who makes a secret of his magic art and steals quietly into the druggist's shop for a pennyworth of castor oil and syrup of buckthorn, with which he sometimes anoints the dog's nose and at other times drenches him, preserving an air of mystery 
that often imposes upon the spectators. Whether the anointing is followed up by incantations and genuflexions, or any of those nocturnal sacrifices which induce the fairies to remove warts and moles from young ladies, we cannot say, but should think it probable, and that the credit of the cure must be due more to the sacrificial offerings over the left shoulder, $\& c$., than to the oil and rhamnus, as we know from practical experience that without faith the remedy mentioned is only an aperient.

Distemper may manifest itself at any period of a dog's life, as may measles in the human subject ; but it is commonly met with in puppies from eight weeks to eight months old. It accompanies the process of dentition, and is generally most acute when the large corner teeth or canines are being cut. These teeth are called tushes or tusks, which is a convenient distinction, as it is fair to suppose that all a dog's teeth are canine tceth. They occupy the same position, or nearly, as do the canines in the human being, the tushes in the horse, and the largest and most prominent of the tearing teeth with which the carnivora are endowed.

Dulness and loss of appetite are usually the first symptoms of distemper or any other illness wilh dogs, and a saline or other laxative at this stage will be safe and beneficial ; say-

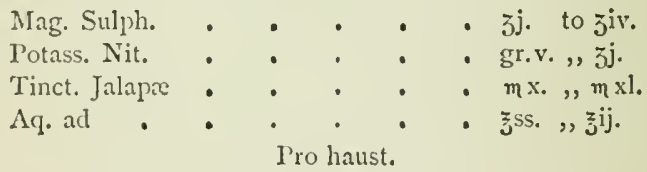

In the course of a few days the symptoms will develop into one of three different forms, in which the respiratory, the gastric, or the nervous system will be most affected, but a blend of two of these forms is by no means rare.

The commonest is that resembling measles, to which infantile affection medical men have most often compared it. In this a fuxion of mucus from the nose, overflow of tears, listlessness, hurricd respiration, cough, and unwillingness to move 
soon combine to make the disease unmistakal)le. It is at this stage that treatment is the most hopeful, and should be directed to the abatement of symptoms rather than seeking for a specific to counteract a poison which cannot be found, or an antidote to something the chemistry of which has not been ascertained.

Many experiments bave been conducted in the hope of determining the specific bacillus of distemper, but without success up to the time of editing this edition of V.C.P. The diffculty appears to consist in a multiplicity of microbes from which the culprit cannot be isolated and cultures procured.

Professor Hobday is still working on the subject, and we have great hopes that he will one day provide us with a reliable serum, or some other form of therapy, by which distemper will be combated successfully.

The dog's eyes and nose should be frequently bathed to keep them clear of the mucus, which dries and becomes a source of pain and irritation. The water for fomentation is improved by a minute proportion of carbolic acid and glycerine-as the former agent should deter the accumulated mucus from bccoming fotid, and the latter is calculated to soothe the excoriated membranes-in proportion of

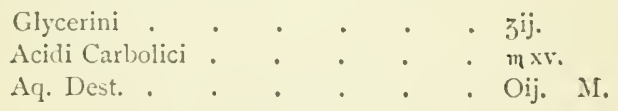

For this lotion, before learing the patient for the night, a substitute consisting of

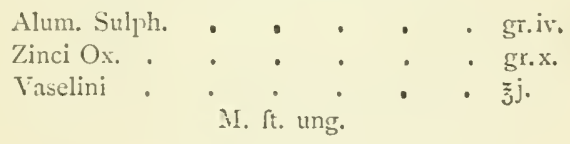

should be applied to the eyelids and nostrils and smeared down the cheeks to prevent scalding of the tears and accumulation of matter and consequent closing of the eyes. The eyes suffer ulceration and various other conditions consequent upon distemper.

Boric acid solutions may be used where acid. carbol, is objected to, or formalin, lysol, chinosol, \&c. 
The before-mentioned remedies are merely palliatives ; but if inflammation of the lungs follows, it is desirable to lose no time in applying counter-irritants to the sides of the chestnot in front, where the thick pectoral muscles cover the chest, but on the ribs from behind the elbow and above it to the last rib. MIustard answers as well as anything in most dogs, but in the long-haired varieties it is not so easily or effectually applied as lin. camph. co. or the preparation of white oils in common use, or-

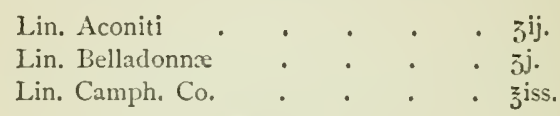

M. ft. lin.

This should also be rubbed into the throat, as there is often much swelling and pain and an inability to swallow. The cough is not, however, due to the throat ; it is not a dry, harsh cough such as characterises laryngeal affections, but is a soft, husky noise, and indicates effusion into the chest cavity or matter within the air-cells of the lungs. Such cases, when not fatal, are often very long about, and are not calculated to bring much honour to the doctor. The best treatment at this stage is to give iodides with regetable tonics. Iodide of potassium scems to produce or excite absorption of the effused matter better than anything else, but must not be given in conjunction with iron. The following is a good formula :-

$$
\begin{aligned}
& \text { Pot. Iodidi . . . + . gr. j. to is. } \\
& \text { Ext. Gentianx } \quad \because \quad \therefore \quad \text { gr. } \frac{1}{2}, \text { ij. } \\
& \text { Quinine Sulph. . . . . gr. } \frac{1}{8},, \frac{1}{2} \text {. }
\end{aligned}
$$

Excipient. q. s. ut ft. pil. Capt. j. nocte mancquc.

The dict should consist of anything the dog will eat, including raw meat. He should be encouraged to move about a little, have his bed changed, and given access to plenty of fresh cool water.

The foregoing is usually the course taken by the disease, and, although there are three pretty well defined forms of distemper, they sometimes merge into one another, and the 
dog with inflammatory lung symptoms may at the same time have dysentery and fits.

The bowels should be kept regular by salines and enemata or ol. ricini, but the latter is not so lasting in its action, and in the opinion of some good authorities is a positive astringent in its secondary effects.

After the urgent symptoms have passed and the dog is getting well, he may have a most distressing skin eruption coming up in great blotches or blains, giving out a sanguineous matter and drying up, or, if neglected, coalescing with other such spots and forming a large raw surface. It would seem to be an exertion or effort of nature to get rid of effete material over and above what the kidneys and other excretories are capable of performing, and is best treated by local antiseptics and internal oxygenation, if such a term may be used. Whether or not this theory be correct, certain it is that no remedial agents meet with such success as large and frequent doses of chlorate of potash; a small dog may have five grains three times a day, and a large one fifteen. An ointment should be daily applied to the sore places as follows :-

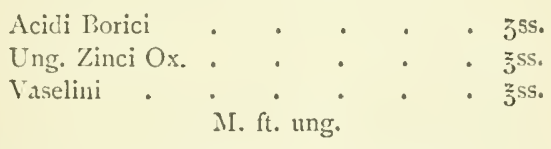

A liberal diet with boiled green regetables will be found desirable.

The chlorate of potash treatment is improved by a little pot. nit. and carbo lig., say one to five grains of pot. nit. according to the size of $\mathrm{dog}$, and the same or a little more of the charcoal.

The gastric or intestinal form of distemper shows itself in sickness, purging, wasting, and prostration. In a certain sense it is its own remedy, and the morbific matter is thrown off in the stools ; these, however, become bloody, the mucous membrane of the bowels is cast off in patches, and a condition similar to typhoid is established. The stomach loses power of digestion, 
and the intestines of absorption. Brunner's and Peyer's glands and the first part of the intestine generally become highly inflamed, and blood and rnucus are constantly passed till the $\mathrm{dog}$ dies of exhaustion if no remedy is found capable of stopping it. Many have been tried, including, of course, the universal panacea of ol. ricini and syr. rhamni, also Aithiops mineral, antim. nig., antim. tart., ipecac., chloride of sodium, Stc.

Careful investigation and experience prove that the successful treatment of this form of distemper is to modify without stopping the diarrhœa, to alter without arresting the secretions, to sheathe but not plug the intestines, to take away the labour of digestion by providing readily assimilated foods, and to surround the animal with such comforts and care as would from time to time suggest themselves to any thoughtful and humane person.

To modify the diarrhœa, small doses of alum. To alter the acid secretions, small doses of sodæ carb. To sheathe the abraded membranes and protect the glands, bismuth subcarb. and glycerinc. To reduce the labour of digestion, beef-tea, milk, and slops. The following mixture will do well in such cases as we have just described :-

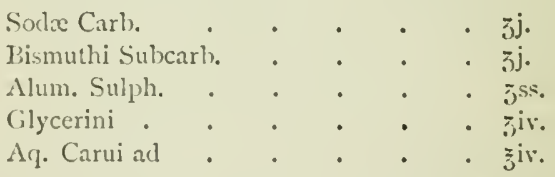

M. ft. mist. Dose-10 minims to I drachm every hour.

If powders be preferred, the following will be found suitable:-

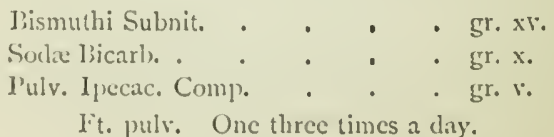

'The nervous form of distemper is the worst to treat, and, although it is reasonable to suppose that the fits and palsy are 
in the first instance caused only by the cireulation of impure blood to the nerve-centres, yet an examination of numerous subjects would show that only a short time elapses in Froducing those degenerations of the spinal cord which leave a dog with 'the jumps' (chorea), or palsied, or subject to fits for life. Treatment, except in the early stage, is of very little use, and should then be carried out with a view to divert blood from the spinal cord to the surface or intestines, or both. The application of a powerful irritant from the back of the head to the loins, such as mustard and acetic acid, or lin. camph. co., or ol. tereb., should not be delayed, and saline purgatives should be constantly administered. All the advantages of bromide of potassium as a sedative may be ensured to the irritable nervecentres by giving it in small doses in conjunction with the salines, giving the dog moderate exercise, aroiding excitement, and looking carefully to the mouth to see if there are any teeth requiring to be removed. The following mixture is recommended :-

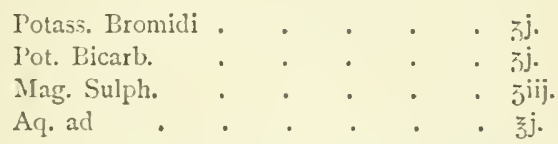

M. ft. mist. Dose-from to to 60 minims every four hours.

The successful treatment of distemper depends more upon good nursing than medicine. The dog should be kept in a well-ventilated dry room, an even temperature being more desirable than a warm one; fresh air, clean water, and nourishing, easily-digested food must be plentifully allowed. Exercise had better be refrained from till strength returns, and water-dogs on no account should be allowed their favourite pastime.

The nose and eyes should be frequently sponged, and in case of entire refusal of food, soups, broth, \&c., should be forced. The combination of a little port wine-about a teaspoonful-with the food, two or three times a day, will be found beneficial.

Besides the medical treatment already recommended, an 
emctic may be given at the outset, antim. pot. tart., in doses of from two to four grains, in butter, or on a small piece of meat, being the best. The bowels must be kept regular by mild aperients, and for this there is nothing much better than the mixture of equal parts of castor oil and syrup of buckthorn -dose $5 \mathrm{ij}$. to 5 viij.

When the more acute symptoms have subsided, the debility may be combated with stimulants and tonics. Port or sherry wine should be given daily with the food, and some of the following medicines administered:-

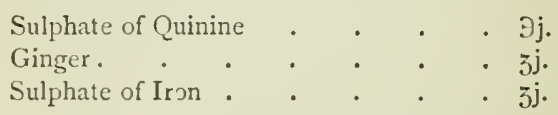

Extract of gentian, q. s. to form twelve pills. One, two or three every day. Or -

$$
\begin{aligned}
& \text { Quininæ Sulph. . . . . . 引j. } \\
& \text { Carb. Ammon. . . . . . zij. }
\end{aligned}
$$

Extract of gentian, q. s. to form twelve pills. One, two or three every day, Or for debility with diarrhaa -

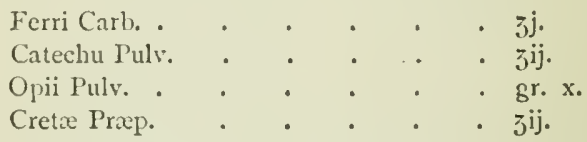

Make into twelve pills with conserve of roses. One, two, or three every day.

Chorea.-This sequel of distemper is one of the most difficult of all diseases to treat, and has baffled the best canine surgeons from all time. It is apparently an interrupted or intermittent supply of nerve force to the muscles, and great benefit has sometimes arisen from the use of the galvanic battery applicd twice daily for a long period. 'The hair on the dog's body should be thoroughly wet before applying the instrument, and the shock made increasingly powerful from time to timc.

Sometimes cases have benefited by giving small doses, as if of a grain, of strychnine daily, while others, holding the theory of irritability of the spinal cord, prescribe sedatives, as 
pot. bromid. from 2 to Io grs., chloral hyd. $\frac{1}{4}$ to 3 grs., cxt. cannabis ind. $\frac{1}{20}$ to $\frac{1}{4} \mathrm{gr}$. We have known good results from each and all ; also from blistering the spine with ol. tereb., and repeating every week or ten days. Arsenic in 2 or 3 ml doses of Fowler's solution has recently been cmployed with great success.

Chorea may occur quite independently of distemper. Occasionally we meet with a case of general chorea, i.c. twitchings of the muscles all over the body; but usually the affection is partial, implicating merely a group of muscles, as of the face, neck, or a limb. The movements in chorea cannot be mistaken for anything else; they are involuntary, and best seen when the animal is quiet. Chorea may be the consequence of irritation from worms or diseased teeth; it may, it is said, follow an injury to the head, but most frequently it is a sequel of debilitating discase, especially distemper. Treatment is by no means satisfactory; some cases get well; but whether we should say they were cured, or that they recovered, is doubtful. Numerous drugs have been tried, and with perhaps equal success. Ether, ammonia, valerian, asafœtida, quinine, and strychnine, salts of iron, copper, zinc, silver, and arsenic, have all found strong advocates.

Spinal meningitis must not be mistaken for the nerrous form of distemper. The symptoms greatly resemble it, and chorea not infrequently results. (See Canine Influenza.)

The following medicines are sometimes attended with success :-

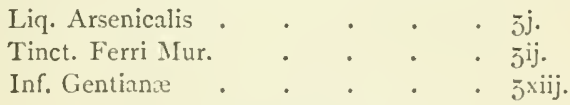

A teaspounful twice a day.

Or一

Zinci Sulph. or Z. Valerian. .

Quininie Sulph.

gr.vj.

¡j.

Confection of roses to form twelve pills. Two every day.

Mange in the dog is of two kinds, though all skin diseases in this animal are commonly spoken of as mange. 
The common or sarcoptic mange is caused by a parasite (Sarcoptes canis), which very rapidly burrows and spreads over the dog's body, and may begin at any part ; its rapid diffusion and the intolerable itching, as also its easy cure, distinguish it from the other form of mange, which we shall presently speak of. Its analogue in man is 'itch,' and its cure may be effected in the same way, for sulphur being fatal to the parasite may be considered as a specific. Dirt and poverty do not actually cause mange, though favouring its contraction. It must be conveyed by contact with another dog, or lying upon the same mat, rubbing posts, furniture, \&c. The remedy that will the soonest destroy the parasites and execute repair in the tissues will bring most kudos to the prescriber. Nothing answers better than the old recipe Professor Simonds recommended to his pupils, because it removes the superficial layer of skin; one or at most two applications at an interval of three or four days will prove effectual.

$$
\begin{array}{lllllll}
\text { Ol. Picis } & \cdot & \cdot & \cdot & \cdot & \cdot & \cdot \\
\text { Ol. Olivæ } & \cdot & \cdot & \cdot & \cdot & \cdot & \cdot \\
\text { Ol. Terebinth. } & \cdot & \cdot & \cdot & \cdot & \cdot &
\end{array} \text { Martes æq. }
$$

A scurfy condition follows the use of this lotion (desquamation of the cuticle), but the hair rapidly grows again and is even improved by the stimulus that the roots have received.

An ointment is sometimes asked for, and therefore we give the following :-

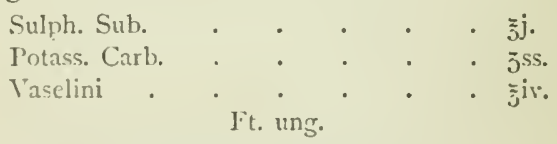

The other form of mange is slower but more persistent, and manifests itself by rubbing the spine under chairs, breaking the hairs along its course. It is often very slow in its development, but crentually spoils a dog's appearance, as well as renders him very uncomfortable. It is caused by a parasite which sclects the skin glands which are largest-namely, those "aprable of leeing erected when the animal is angry. Its name 
is Dimodex folliculorum; it is long and narrow, witl eight legs of a rudimentary nature on the thoracic portion, and is found inead-downwards in the follicles. The following is good :-

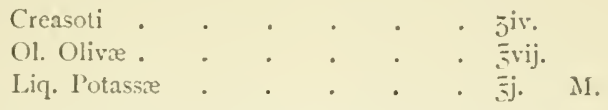

The hair should be closely clipped and the above application well rubbed in on alternate days for a fortnight.

This form of mange is by no neans uncommon, yet often not recognised till it has gained a firm hold, the rubbing being thought to be a disagreeable habit and its real cause not suspected.

Eczema is a common disease among dogs, and often very troublesome to treat. It is commonly mistaken for mange, though having for its cause quite a different origin, and the remedies having nothing in common. It comes on usually without any preliminary symptoms, though a very careful observer might notice feverishness and restlessness before the skin becomes reddened-which is conmonly the first thing noticed - all along the belly, thighs, under the arms and other hairless portions of the body. This intense redness is followed by the appearance of numerous small resicles or bladders, which vary in size in different attacks, and when associated with distemper often have a distinct character of which we will presently sperk.

The vesicles contain a thin, watery fluid and not pus or matter; they break, coalesce, and form scabby masses, which often cause further irritation to the surrounding sound skin, and so produce a confluent mass which may easily be mistaken for a burn or scald, but which had its origin in the resicular eruption known as eczema.

It should be cleariy distinguished from mange either of the sarcoptic or follicular variety; the latter being both parasitic in their origin and only to be cured by remedies that will destroy the living 'varmints.'

It is not infectious or contagious, and as often as not 
originates in a kennel to which no other dogs have had access, and where the sanitary arrangements are good. That all the dogs under one management or ownership may have it at one time is no argument as to infection, but rather a proof that like causes produce like results.

Indigestion or errors of diet account for nearly all attacks of eczema, though many 'doggy' men are difficult to convince on the point, and much acrimonious correspondence has been produced by reterinary surgeons objecting to making any one dog-biscuit the sole diet for a long period. Most house dogs are over-fed. Besides his regular meal, or meals, a dog will certainly find some extras for himself if he gets the chance of running about loose, and the cook or the children cannot be prevented from providing some tit-bits, generally of an indigestible character, for their canine friends.

Couch grass (triticum repens) is the one extra which the dog may eat ad lib. Instinct will lead the animal to it if there is any on the premises, and if the owner of the dog has no garden, any friend who has one will willingly give away all the couch grass he has if the applicant will only take it away by the roots - a very difficult matter. Dogs will eat it in their kennels if supplied with it freshly gathered.

Eczema is not infrequently the result of a too limited dietary, and sometimes an entire change of food from whatever has been given is the best remedy; we have known eczema cured with no other remedy than horse-flesh, and have seen a whole kennel made ill by a liberal supply of milk.

There are a great many theories and no little prejudice in this matter of dog-feeding, and we think, as the result of observations extending over thirty years, that dogs thrive best on a mixed diet ; this notion is supported by a compromise betwcen nature and art. Nature provicles dogs with teeth of a character destined only for flesh-eating, while domestication makes it undesiralsle to give meat alone.

Eczema in the dog appears to be accompanied with general acidity, and alkaline bicarbonates and saline aperients hare proved the most valuable remedies. 
In a simple case we should advise an aperient such as the time-honoured syrupus rhamni and ol. olive, rather than ricini, which is such a bother to administer, followed up with such at mixture as the following :-

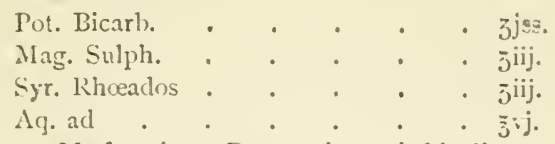

M. ft. mist. Dose- $3 \mathrm{j}$. to $\mathrm{z}_{\mathrm{j}}$. bis die.

To allay irritation, and prevent the dog from scratching and wounding his own skin, a bath daily, of glycerine I part, aqua 60 parts-or, if he be a long-haired or delicate dog, not fit for the bath, a lotion of the same, sponged on to the affected parts - may be used. To this may be added potassa sulphurata, $5 \mathrm{j}$., if the simpler lotion fails.

Chronic eczema is more difficult to trcat, as it too often happens that the subject is a pet dog, indulged with sugar and pastry, or 'stuffed' with meat to the exclusion of all other food. In addition to a change of food, and a course of salines, as previously recommended, we would say carefully wash and gently dry the patient with a soft towel, and then smear him well with ung. zinci ox., made softer and thinner than the B.P. preparation by the addition of ol. olive, I to 3 or 4 parts of ung., or an oily application may be used of liq. plumbi I part, ol. olive 50 parts. A cooling dressing is the following :-

$$
\begin{aligned}
& \text { Liq. Potassæ . • • . . } 5 j \text {. } \\
& \text { Ol. Olivie . . . . . . zix. } \\
& \text { Liq. Plumbi . . . . . 3iv. } \\
& \text { M. sec. art. }
\end{aligned}
$$

Some very bad as well as chronic cases have been quite cured by a persistent use of

$$
\begin{aligned}
& \text { Acid. Carbolic. . . . . mx. } \\
& \text { Ung. Zirci Ox. Penє. . . . } \bar{j} \text { j. } \\
& \text { II. ft, ung. }
\end{aligned}
$$

A bad case will require a pound or two, but persistent daily, inunction of large quantities is worth trial for two or three 
months (in the case of valuable dogs) when all other agents have failed.

Chronic cases sometimes yield to arsenic, in the form of liq. arsenicalis $m j$. to $m$ iij. daily, and this may be given with food or drink, or dispensed with aqua as a mixture.

An eruption resembling eczema is not uncommon in pups debilitated by distemper ; it is not, strictly speaking, eczema, but seems to be an effort on the part of the skin to get rid of deleterious matter. It comes up on the inside of the thighs in large watery bladders, which develop into pustules, and leave a pit when they rupture. It very nearly resembles small-pox in its periods of papulation, vesication, pustulation, and desquamation, and requires emollient treatment locally, and mineral tonics internally.

For the comfort of dogs suffering from skin-diseases, it should be remembered that straw, shavings, \&c., form very irritating and comfortless beds; an old rug or soft garment should be provided, and afterwards destroyed.

Eclampsia.-Bitches are subject to a form of parturient discase called eclampsia. It is generally the result of suckling too many pups, and the symptoms much resemble poisoning by strychnine. There are tetanic spasms, rapid breathing, and the owner describes the animal as in a fit. The treatment consists in removing some of the pups, giving a sedative, as bromide of potassium to to 60 grains, a brisk aperient, nourishing dict, and cxercise.

Constipation in the dog is much more frequent, we had almost said 'natural,' than in other animals. The faces of a healthy dog should be somewhat hard, and no heed taken of a moderate amount of straining in defacation. It is just as natural for a dog to put himself into an attitude both anxious and ridiculous when defrecating, as for a horse to make so much ceremony before staling.

Just inside the anus are certain glands which emit an unctuous matter when pressed upon by the hardened freces and the muscular efforts of the animal to pass the dung. 'They 
can best be compared to that part of a goose or aquatic bird which is known as the 'Parson's nose.' It is a provision against constipation, while hardened fxces are normal and right in the case of these animals.

If, however, a dog is observed repeatedly putting himself into these attitudes, and nothing else is observed on going up to the spot, it is time to give him a little help. This is best done with an injection of soap and water, but as it often happens that the prescriber cannot spare the time and the owner will not take the trouble, resort is had to a mixture of syr. rhamni et ol. ricini, partes æquales-dose of the mixture from $\frac{1}{2} \mathrm{oz}$. for a smail terrier to $3 \mathrm{iij}$. for a mastiff or other very large animal. Constipation in the $\operatorname{dog}$ is very frequently caused by eating bones-not gnawing them-like those of mutton-chops, game and poultry, which are easily crushed and more easily swallowed, and these make the light-coloured, dry, hard freces which used formerly to be collected at streetcorners for dyer's purposes.

Youatt recommends an aloetic ball; but there is a great objection to aloes, as dogs are so easily nauseated, and one does not care to give a ball more than once. Castor oil also necessitates a bath afterwards in long-haired dogs, whose muzzles are rendered sticky and wretched, if no greater failure occurs in administration. Mag. sulph. is an aperient preferable to all others, and this should be given in several small doses rather than in one heroic drench.

The following is a suitable mixture for habitual constipation :-

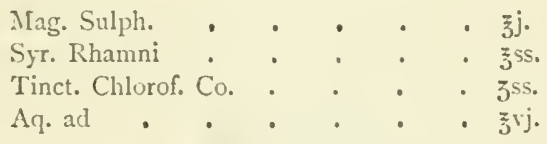

M. ft. haust. Capt. इij. ad $\varlimsup_{j} \mathrm{ij}$. omni mane si opus sit.

Some green food, as boiled cabbage, spinach, \&c., mixed with gravy and table refuse, should be recommended, but not much, for we have in our minds, while writing this, the case of 
more than one lady whose pet has become intolerable from the evolution of gases at both ends of the dog.

The following is a good aperient pill :-

$$
\begin{aligned}
& \text { Pulv. Jalapæ } \\
& \begin{array}{c}
\text { Pulv. Cambogire } \\
\text { M. ft. pil. Pro re nata. }
\end{array}
\end{aligned}
$$

It has been pointed out elsewhere that powders are the easiest form of medication for dogs, and a simple aperient of Io to 20 grains of pulv. jalapæ may be thrown upon the tongue. It may be fortified with a grain or two of calomel, where the pallor of the fæces indicates inaction on the part of the liver. Dogs can bear onc fairly large dose of calomel, but are prone to salivation when repeated. It may in fact be said that where 5 grains of calomel would be quite safe as an aperient, ten doses of half a grain each would very likely induce mercurial poisoning.

Jaundice.-This is not infrequently a sequel of distemper, though it also occurs in old dogs, and quite apart from the disease, or host of diseases, classified under the generic term of distemper.

This malady should be readily recognised, yet we are in a position to say it seldom is until it has made serious inroads on the dog's constitution; his attitude and demeanour report headache, nausea, languor, loathing, disgust, more plainly than words, and his mute eloquence must appeal to the veriest doghater.

If the white of his eye (conjunctival membrane) is examined, it will be found yellow in colour, or even dark orange in tint, and this cxtends to the skin. If the nude parts are examined and the inter-digital spaces, the colour will at once tell the prescriber what he has to deal with.

Sometimes a number of hounds will be affected with this complaint at one time, and then it may be traced to crrors of diet or enviromment, but in the cases brought under the notice of the everyday practitioner it is generally due to an idle life, indulgence in rich food, and, more than anything 
else, to lying in front of a fire persistently. The treatment must be bold and energetic, though Mr. Steel, in one of the latest canine works, does not seem to think so. If the disease is not grappled with at once and heroic remedies administered, the patient will be dead before the milder system has had time to be tried. Dogs, like babies, can take very large doses of calomel, and we look upon this agent as our sheet anchor. $\mathrm{We}$ are not afraid of ten grains for a small dog or twenty grains for a hound or any other of the large breeds - not to be repeated, of course. It is not the bold dose that brings about salivation; it is the repetition of small, and for this purpose ineffectual, doses. Salines may be given afterwards. Mag. sulph. $5 \mathrm{j}$. to $5 \mathrm{iv}$ with inf. quassize does well. Exercise should be enjoined, light food, such as milk puddings, fish (beware of bones), lunch biscuits, \&c. Dogs need not be drenched with food if they refuse it; there is no domestic animal capable of such long-continued abstinence as the dognot even the cat. Fasting cats get in at open larder-windows and snap up ' unconsidered trifles' at hours when a respectable dog is on guard duty. Cats sometimes suffer from jaundice, but-alas for the harmless necessary cat !- her ailments are not generally observed till too far advanced for any remedy but acid. hydrocyanic. $3 \mathrm{j}$. secundum artem, unless she sneeze or cough, when she is hustled out of the house under the impression that she is going to be sick. We owe poor pussy some reparation; she is a social martyr because not generally understood.

Rheumatism in Dogs.-Veterinarians are agreed that dogs are unquestionably subject to rheumatism, and that they are, of all domestic animals, the most liable to it. In their case it is not so frequently a joint affection. More usually it is the muscles of the chest and shoulders, and sometimes of the loins, where the attack occurs. It comes on very suddenly and is more amenable to treatment than in man. It is called 'kennel lameness' among hounds, and is known to most sportsmen. Lapdogs and all other breeds are subjects of it, 
whether exposed to bad weather or not. It is not very difficult to diagnose, as the patient cries out on being lifted by the forearms or goes rery stiff-all in one piece-when lame in the loins.

Dogs are also liable to rheumatic fever. It does not often take this form, but the subjects of it are the most piteous objects. Curled up in a heap, unable to move without crying out with agony, with a very high temperature, and an odour something like that of a kennelled fox.

Salicylates, in doses proportioned to the weight of the individual sufferer, have proved the most suitable remedies, but very few dogs get the necessary attention. All animal food must be withheld and a diet of milk and light puddings prescribed; spoon feeding during some part of the illness.

Treatment of the common forms of rheumatism.-Put the dog on a milk diet and apply daily the liniment referred to on p. $\mathrm{I}_{3}$ for horses. Give the following mixture :-

$$
\begin{aligned}
& \text { Vin. Colchici . . . . . miij. } \\
& \text { Sodæ Salicyl. . . . . . gr.r. } \\
& \text { Aq. ad • . • . - . jj. } \\
& \text { II. ft. dusis. lis die. }
\end{aligned}
$$

Rheumatism scldom settles into the chronic form in dogs, but may become frequently remittent, and the mixture and liniment should be kept in stock for such subjects. These are the cases in which a veterinary prescriber may cover himself with glory and get appointed as canine surgeon in ordinary. The rheumatic subject should not be washed in the ordinary way, but a medicated bath may be prescribed in the summer months secundum artem.

The doses we have given are for a terrier, and may be doubled for a sporting dog, and trebled for the large breeds.

A dose of mag. sulph. from $\bar{j} \mathrm{j}$. to $\overline{\mathbf{z}} \mathrm{j}$. should be prescribed where there is constipation, and it need hardly be added the dog should be kept out of the water.

Gastritis in Dogs is often the result of over-fceding, or of the presence of irritants in the stomach, splinters of bone, 
especially fish-bones, becoming lodged in the lining membranc. Frequent vomiting of almost clear water is a prominent symptom, and inability to retain any food, marked thirst, and rapid loss of condition. In bad cases the dog will often seck a cold stone or pavement, and stretch out upon his belly, as if to cool the burning sensation he doubtless experiences.

In the dog we have a patient more amenable to treatment than horses in a similar complaint. Severe cases of gastritis often yield to a very few doses of

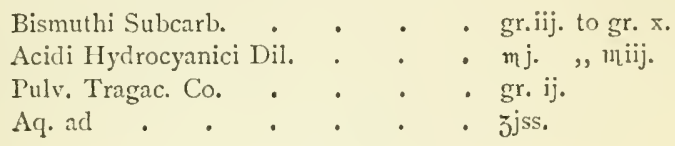

Ft. dosis. 4 tis horis sumend.

The patient should have abundance of ice-cold water supplied him, or ice-water spooned down every hour, with 5 to ro minims of brandy in very bad cases.

No solid food should be given, but milk and milk puddings, gradually increasing the quantity, and by degrees reverting to his former diet.

Cats show much the same symptoms, and the treatment above suggested is equally applicable, modifying the dose to the comparatively small animal to be treated.

Canine Influenza (so called).-An epizootic has appeared during the past few years among dogs and has gained the name of influenza. It never assumed the catarrhal form as with human beings, but began as gastritis, and, after every sort of remedy had been tried, an eminent professor discovered (?) the advantages of the bismuth and hydrocyanic treatment advised in this work for gastritis when it was first published, and which stands above.

Like its congener in the human subject, it later on took the form of meningitis (see also Chorea, p. 236), with symptoms of 'nerves,' inability to stand still, and uneven progression advancing to actual paralysis. This form is best treated with 
spinal sedatives, preferably bromide of ammonium or small quantities of chloral together with vegetable bitters, none of which answers better than extractum gent.

\section{Worms in Dogs.-Of the varieties of tapeworm infesting} dogs, the commonest is the Tania marginata. Dr. Cobbold estimates its presence as in 25 to 30 per cent. of dogs in England, 44 per cent. in Denmark, and 75 per cent. in Ireland. Sporting dogs, as a rule, suffer more from tapeworms than house dogs and pets, as the larval or cystic form of some varieties infests the entrails of hares and rabbits, the 'fifth quarter' of which is often the dog's share of the spoils.

Another variety, called Bothriocephalus, is found in fish, and seaside dogs and cats suffer most from these. The dogs of Norway and Sweden, whose masters live almost entirely upon fish, are hardly ever free from this kind of tapeworm. The cat who follows the fishmonger's barrow, and derours the offal during the cleaning of fish, is a very frequent victim, and wastes away to a shadow without the cause being, as a rule, ascertained. Cats are most difficult animals to treat, and, owing to their extremely scientific ideas of sanitation, it is often impossible to tell whether medicines have acted upon them, except by the improrement to be noted afterwards.

All the tapeworm class can be ejected by pulv. areca, and that agent is worthy of the faith that was at one time placed in it. If it fail, it is because the powder is old and has lost its 'virtue.' Dog-dealers and others are fully aware of this, and prefer the difficult task of rubbing the nuts on an ordinary nutmeg-grater to buying the finely levigated article of the shops.

$\Lambda$ dogs are of all sizes, from the nude little terrier whose owner prides himself on being able to put him in a quart pot, to the massive St. Bernard or great Dane, one cannot put down a dose without some sort of sliding scale; and this Dr. Cobbold, the celebrated helminthologist of the Royal Veterinary College, clid by advising two grains for every pound weight of the dog. The practice of giving worm medicines en an emplty stomach 
has the sanction of long practice, though remedies often take effect when given in food-a fact worth bearing in mind if the animal wears a forbidding expression and the owner is unwilling himself to run the risk. The dose should be repeated at intervals of a few days, as some worms are most tenacious of life, and can 'brokenly live on' just as well as if entire; indeed, the tapeworm class differs from all others, inasmuch as any two segments can impregnate each other, being biscxual and capable of producing some 30,000 eggs.

Ol. filicis can seldom be retained long enough to be effectual, hut a very good pill made of the following ingredients is often used with success, and, as it is not always known from what particular kind of worm a dog is suffering, it is worth while to give him a charge that will hit either :-

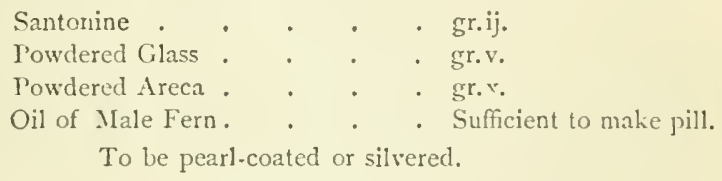

The round worms, or ascarides, are pretty easily removed by the use of powdered areca nut, in doses, for ordinary-sized dogs, of about one drachm, made into a pill.

The powder is rather light and bulky, so for small dogs had better be made into two pills, and given one after the other. The areca nut should be given over night, and followed by a dose of about an ounce of castor oil the first thing in the morning. To make sure of removing all the worms an animal should have at least two doses given at an interval of a week. In place of areca nut, worm seed or santonica may be used. The dose of the powdered seed for a medium-sized aged dog is about six grains, given as a pill in the same manner as areca nut.

'Stonehenge,' in his work on the 'Greyhound,' recommends Indian Pink as a vermifuge. An infusion of half an ounce, in a pint of boiling water, to be given, when cold, at night, and followed by castor oil in the morning.

The tapeworm is by no means sp easily remored. This 
parasite attaches itself to the intestine by hooks situated on its head, and as it grows detaches the posterior joints filled with eggs. The presence of these joints in the fæces of the animal corroborates a diagnosis. The chief difficulty is the head; for so long as it remains attached the parasite grows, and causes irritation; sometimes fits.

The ordinary anthelmintics seem to be powerless on the tapeworm. We consider the best to be the oil of male fern, given in linseed tea, or tied up in a small piece of sausage skin. The dose for a medium-sized dog, say a bull terrier, is abo: ht hulf a diachn. It should ba repeated in a week or so, and if the $\operatorname{dog}$ is weak a little tonic medicine is required, Another drug spoken of by some very highly is the Abyssinian kousso. The dose is from four to eight drachms.

If it is decided to give a dog ol. filicis or other agent known to excite nausea, the animal's mouth should be strapped so that he cannot open it, and his head tied up in such a manner that he cannot depress his nose between his legs. This will deter him from vomiting, as he cannot do so without putting his head down.

Fits in Dogs.-Convulsions are most commonly met with in young dogs, and may generally be traced to some irritating cause, as worms in the intestines, or the natural changes in the teeth. Treatment is remedial and preventive.

If the creature is brought to the pharmacy while actually in a fit, the V.C.I. may corer himself with glory by extemporising a cardboard muzzle and causing the animal to inhale chloroform through a sponge. To prevent the return of fits after removing any probable cause, the following pill may be given :-

$$
\begin{array}{llll}
\text { Arsenic. alb. } & \cdot & \cdot & \text { - gr.j. } \\
\text { Ferri Sulph. } & \cdot & \cdot & \text { - gr.xx, } \\
\text { Ext. Gentiana } & \cdot & \cdot & \text { - sufficient to form } 15 \text { pills. }
\end{array}
$$

One every day.

Pot. bromid. gr. xr., combined with chloral hydrat. gr. x., is 
often serviccable in these cases. Order exercise, good food, and a dry house.

Canker.-This is one of the things upon which the countcr prescriber is frequently consulted and should be familiar with, for the patient can be brought to him and treated at his own pharmacy:

The old remedies such as argenti nit., cupri sulph., \&c., cannot be too strongly condemned, and he who would be successful in the treatment should remember the cause and origin of canker and not treat it as he would an ulcerated condition in any other part of the body. The ear of the dog, we may remind our readers, has on its inner surface two kinds of glands which secrete respectively wax and an unctuous matter for keeping the ear soft and pliable. These glands when irritated by dust or water or other agents become inflamed, and instead of lubricating and protecting the parts, they give out an acrid matter which soon again spreads the inflammatory area and ends in ulcerations, which may be of any shape and size, but are oftenest found as cracks. Water dogs such as spaniels and retrievers are perhaps the most frequent subjects, but all sorts and conditions of dogs are liable to it.

Instead of pouring in irritants and increasing the pain and inflammation, the ear should be softened and the morbid products broken down in the first place by a drachm or two of ol. amygd. dulc. dispensed secundum artem (we choose ol. amygd. as having the least amount of gummy or extractive matter). If this is warmed in a teaspoon over the gas-jet to about the tem. perature of the body and poured in gently, the dog will not resent it. This is most important, as first impressions go a long way with a dog. If frightened by the first dressing, or the irritants previously used, he will always be a troublesome dog, and never a jolly dog, to treat. This done on two or three successive days, the dog should be firmly but kindly held while the meatus is cleaned out with cotton wool on a bone penholder or blunt forceps, care being taken to remove all the débris without injuring the sensitive lining of the ear. 
The following simple lotion should then be used, warming as previously advised :-

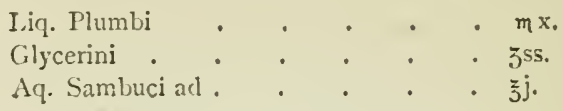

M. ft. lotio. Quotidie applicand.

Or this, which is the most famous among canine practitioners-

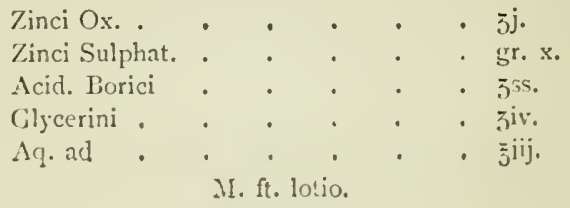

Or the ear may be dressed with iodoform, oxide of zinc, starch, and boric acid, any of which will often cure the disease. Success depends not so much upon the drug selected as upon the manner and frequency of the dressing.

If old ulcerations are present, we recommend, after the clcaning process, no stronger application than

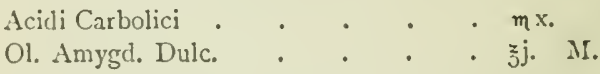

The treatment consists essentially in removing the causenamely, the mal-secretion; and if this can be done the effect ceases. There are some old-standing cases that are incurable, chicfly because the owners will not take the necessary trouble themselves or pay others to do so. The remedies above mentioned will mitigate the pain and subdue the offensive odour if nothing more. External canker is a vague term applied alike to ulcerated flaps and serous abscesses (which sce, p. 270).

In treating canker we should give altcratives, such as daily grain doses of flor. sulph. or the following :-

$$
\begin{aligned}
& \text { Hydr. c. Crctâ . • . . • gr. iij. } \\
& \text { llor. Sulpls. . . . . . gr. xij. } \\
& \text { Carbon. Animal. • . . . gr. ix, }
\end{aligned}
$$

II. ct divide in pulv. xij. j. omni dic. 
Such small and comparatively tasteless powders as these can le given in the food. Patients should not be allowed to enter water, and if pet dogs must be washed, care must be taken to keep the ears dry by plugging with cotton wool.

Deafness.-Dogs are frequently deaf, but there are fewer deaf dogs about since the barbarous practice of cropping the ears has fallen more or less into desuetude. It is to be hoped cropping will altogether disappear, as clubs are beginning to disqualify cropped dogs from taking prizes at shows. There is not much to be done for deafness in dogs unless it is traceable to some recent injury or blocking-up of the ear with wax and dirt. Some of the large-eared hairy breeds of dogs get mats or tags of woolly hair formed on the inside of the flap, making the ear sore by its weight and friction, and by preventing natural evaporation the base of the ear becomes inflamed, and a green offensive pus is produced. The remedy for this is fomentation with warm water and glycerine, careful removal of matted hair with scissors, and the daily application of the following ointment :-

$$
\begin{aligned}
& \text { Ung. Zinci . . . . . } 3 \text { j.j. } \\
& \text { Ol. Amygd, Dulc. . . . . 3iij. } \\
& \text { Lanolini . . . . . } 3^{\text {iij. }}
\end{aligned}
$$

II. ft. ung.

The yoor dog for whom this little office is performed will quickly repay the trouble in his improved appearance and brisk manner, if not by his gratitude.

When deafness is the result of a blow or a fall and comes on suddenly, a brisk aperient, such as mag. sulph. 5j. with tr. jalape mlxv. (for a terrier), will have the effect of diverting congestion.

Slit ears in sporting dogs and gun-shot wounds are common enough, and cause a great deal of pain to dogs, without, as a rule, getting any treatment. They should be gently handled and dressed with the above ointment, unless a shot or thorn is found, when it should be removed. Injuries to the flaps of the ear are often very troublesome to heal, as the patient inflicts 
fresh wounds with his foot in vain efforts to allay the irritability. Bandages upon the head or ears we do not recommend; the only exception we would make is in the rare instance of keeping a pad on a dislocated or seriously injured eye.

\section{Preumonia, or Infiammation of the Lungs. - This} disease is by no means uncommon in dogs. The dog becomes suddenly languid, his nose is hot and dry, he loses appetite, assumes a lying posture, but is restless and frequently moves. At a rather later stage of the disease, he prefers to sit with his head in the air, mouth open, and bears an anxious look on his face. Breathing is short and painful, the cough frequent and dry. His mouth is dry, and his urine is highcoloured. Sometimes a reddish mucus runs from the nose.

Treatment.- The first thing to do is to place the patient in a moderately warm, airy room or shed. A mustard plaster should be applied to the chest, and an embrocation of lin. camph. co. rubbed into the adjacent parts. For internal administration give : vin.ipecac. mv., tinct. aconiti B.P. mss., tinct. camph. co. mx., glycerini ad $5 \mathrm{j}$., pro dosi, bis terve die. This is for a fair-sized terrier; for a St. Bernard twice the above might be given. Inhalations of tinct. benz. co. are found beneficial.

Hot Feet.-An inflammatory disorder attacking the feet of dogs, caused by long runs on hard dry ground. 'The paws become hot, very tender, and are often swollen. Rest, gentle purgatives, light diet, and poulticing, or the application of fuller's-earth and lanoline or lard at night, will soon restore the animal.

Rabies is due to a specific virus. It is the most terrible disease affecting any animals, and it is especially dreaded for the reason that it may be transmitted to other healthy animals and to man by means of a virus contained in the saliva. It appears at all seasons, but cases have been found to be most frequent during the autumn and in the early spring. The first symptoms of the malady are often not very 
marked, so that an animal may be suffering froni rabies for some time before the disease is suspected. The earliest manifestations are those which characterise many other complaints. The dog is listless, and appears sad; often he has no appetite ; he sceks darkness, and keeps in his kennel or bed. 'The special feature which suggests danger is the fixed gaze, the eye being brighter and redder than usual. The dog will often lick cverything near him, and may perhaps be seen lapping his urine. If he has maintained his appetite, he loses it later. He does not bark, but whines with a peculiar and characteristic raucous cry. He still obeys his master, but not eagerly, and at times he flies at imaginary objects. It is not the case, as is often said, that he shows terror at the sight of water. On the contrary, he will go to the vessel and try to drink, but, as the disease progresses, deglutition becomes impossible. He bites at his bed, at straw, at wood, at rags which he can get hold of. If chained, he bites at the chain. If not chained, he will try to escape. Even when the disease has gone as far as this, he will seldom bite his master, but if he escapes he may bite any other dog or person whom he may meet. Within eight days, if he be not previously killed, paralysis supervenes, and death relieves him from his agony. There is no known remedy for rabies. If the symptoms we have described are exhibited, the dog should be kept chained in a place by himself, and a veterinary surgeon should be called. If he confirm the suspicion, the sooner the animal is killed the better.

'There is a form of rabies known as 'dumb rabies,' in which the dog's jaws appear to be paralysed. This form is very frequent and does not seem to arise so much from paralysis of the jaw, which is kept open, as from swelling of the fauces and back part of the tongue, which makes it difficult to close the mouth. The dog is not however dumb, but gives vent to occasional howls quite different in tone from his ordinary voice. His mouth is dark, red, and dry, from evaporation through keeping it open. He cannot bite, but the attendant must beware of supposing that he has a bone in his throat and trying to take it out with the fingers, for the saliva is as 
poisonous as in the other variety. There is no remedy, and the dog will soon die.

Canine Dentistry - Many chemists who extract teeth may have overlooked the fact that dog patients also often re. quire surgical aid.

Perhaps some reader has received an early and unfavourable 'impression' of dog's teeth, and the idea of turning them to profit has not entered his head. To such we offer a few remarks as to how to secure the patient with safety and comfort. Fortunately for canine surgeons the largest dogs have generally the mildest dispositions, and the 'baying of the deep-mouthed hound' is usually the worst part of him. Bites come more frequently from the pampered pet, whose dector injudiciously approaches him upon his mistress's knee instead of insisting on a stranger holding him. Only those who frequently handle dogs realise that they more often scratch than bite.

When some minor operation has to be performed upon the mouth of a smail dog, he should be placed upon the lap of a man or boy, who should hold the hind and fore leg of one side in one hand and the hind and fore leg of the other side in the other hand-this will leave his head free, but confine his body within reasonable limits and prevent him from scratching. l'lacing an old bonnet string or piece of webbing in the mouth, draw down the jaw by holding the two ends together and close to the dog, while with the right or free hand you use the forceps or scaler; with a very little practice and firmmess this will be found easy.

$\Lambda$ rather larger dog may be wound round with a large towel or bulky wrapper to prevent the free use of his hind legs, while the front ones are held as before. If a very large dog, he will have to be cast by a cord attached to his collar and wound round his hind leg, so as to pull it forward till it meets the collar; in this way you can make him contribute to his own security by being unable to get up. The jaw may be kept open in the same way or by a wooden gag.

The method of operating under chloroform is rather dan. 
gerouts, and the most experienced rets. sometimes snuff out the patient. The subjects to be avoided are fat, old, and petted dogs, which frequently suffer from fatty degeneration of the heart.

Dental troubles seldom begin till the puppy is three or four months old, when he may be subject to fits and other nervous disorders, which are frequently cured immediately by the removal of the temporary teeth, which can be readily distinguished from the permanent; there is usually very little. trouble in extracting them, as after the fourth or fifth month the fangs are absorbed as in the human subject.

The most frequent time for fits arising from teething is from the fifth to the seventh month, when the tushes, or long corner teeth, come through; for some reason not understood the temporary and permanent tushes are more often seen together than any other of the dog's teeth, and the primaries should then be removed. Puppies frequently remove them without assistance in that game of 'French and English' they are so fond of playing with one's farourite slippers or the hall mat, if nothing more valuable falls in their way. Large bones should be advised for the same end. Small ones are too often crushed, swallowed, and impacted in some part of the digestive canal.

The dog suffering from toothache rests his face on a cool stone or leans it against a wall and whines, striking the side of his mouth with the hind foot.

Extraction is, of course, the remedy for diseased teeth, as also for that dreadful condition of the mouth known as canker, in which the teeth become ankylosed together by a foul growth of degraded material, causing ulceration of the gums and dribhling of saliva, accompanied by the most intolerable smell. All the diseased teeth should be removed, as the necessity for artificials is not so great in dogs as in man; the gastric juice of the former has twice the digestive power of that of man, and it is in accordance with the nature of the dog to bolt his food, his teeth being used only to kill his prey or tear up the food, not grind it as with ruminants or solipeds. Ages of domestication have modified the dog's teeth as well as his digestion, 
but a dog can live and thrive on soft food very well, without any teeth whatever.

The appearance of tartar on the teeth commences usually at about fifteen months, and the dog's age can be fairly well guessed by an expert by this means alone. When it accumurates to any extent the teeth should be scaled, or ulcerated gums and unpleasant breath will bring about unmerited banishment of my lady's pet from my lady's chamber. The same tooth powders can be used as for human beings, and in many chronic cases the use of charcoal biscuits is a benefit alike to the dog and the owner.

Splinters of game and poultry bones are not infrequently found firmly imbedded between the teeth, and occasion much distress; the dog strikes the sides of his face with his front paw and dribbles at the mouth. A timely removal with the stump forceps and a little astringent wash applied, or rather champed, on a sponge will soon effect a complete cure. 


\section{TREATMENT OF EIE DISEASES}

THERE are diseases of the eye special to some animals, and others common to all. No one should trust himself to treat diseases of the eye without a fair acquaintance with the structure and functions of the organ of sight. Such knowledge may be obtained from cheap elementary works, such as Huxley's 'Physiology,' or Kirke's ; and we may add that the dissection of a bullock's or sheep's eye is well worth the trouble, though the experimentalist may have no intention beyond that of prescribing for such simple ailments as are commonly brought under the notice of the counter prescriber.

The eyes of animals differ only in a few particulars from the human eye, and to these differences we will briefly call attention:- The position in the face, finding its most conspicuous example in the hare, which looks behind her; the lashes and brow; the retractor muscle which enables animals to draw back the eye into its socket, and the greater development of the haw or caruncula lachrymalis. The tapetum luci$d u m$ also, which enables animals to graze in a very low medium of light, and cats to 'sce in the dark,' as is commonly said. It is not true that they can really see in the dark, but they can do so in a very low medium of light. An absolutely blind cat can find its way about without running into objects of furniture, \&c., but this is chiefly owing to the 'whiskers' or long feelers with which pussy is endowed; at the base of these feelers is a little cauliflower-like expansion of nerve which gives the most delicate sense of touch. Bats have the sense of touch so highly developed that a blinded bat can fly about 
a room in which silk threads are suspended without ever coming in contact with them. To their highly developed sense of tcuch and a good bump of locality must be ascribed a great deal of the ability cats possess of 'seeing in the dark.' There is, too, a phosphorescent light or luminous carpet at the back of animals' eyes, particularly felines, which enables them to see much better than we can, in anything but absolute darkness. Savages can see better at night than civilised men, and countrymen, gamekeepers, and rural policemen can see where a city man feels absolutely helpless. These are of course only examples of the fact that senses not fully developed fall into abeyance, or by exercise develop proportionately.

Much of the expression of the face depends on the eye, though it can hardly be said to be the 'window of the soul' as in man. Man does not lay his ears back when angry, but the expression, for instance, of a vicious horse or cat is as much due to the ears as to the eyes. Horses are commonly suspected of vice if much of the white of the eye is visible, but it is not an invariable rule, nor is a deep hollow over the cye an invariable sign of age. It may sometimes be seen in a quite young horse, when one or both of the parents were old.

Inflammation of the Eyes (Ophthalmia) is not unfrequently caused by the lodgment of foreign bodies, and was more common formerly than now. Hay-racks were then above the horses' heads, and in pulling out the provender, seed would fall on the face and into the eye. Stables were dark, and the atmosphere saturated with ammonia. The blind horses to be met with thirty or forty years ago were probably more than double the number at the present day.

Inflammation is caused sometimes by the lash of the whip, and may be traced long afterwards by a white mark being left upon the eye.

The first thing to do in treating an inflamed eye is to ascertain if there is anything lodged in it, such as seed, chaff, or grit. Warm fomentations with extractum belladonne are of much service in reducing the swelling, but no such prepara- 
tions as zinci sulph. should be used during the inflammatory stage.

The introduction of cocaine into veterinary practice has been of the greatest benefit in treatment of eye diseases and accidents. A ten per cent. solution thrown upon the conjunctiva by means of a c. h. pencil, enables the operator to get a fair view and manipulate the lids in order to remove a foreign body or suture a torn eyelid.

Some practitioners bleed from the angular vein or apply a counter-irritant to the skin over that region. We doubt the utility of such treatment, and there is always the risk of the animal rubbing it and causing further injury. Belladonna has the advantage of keeping the pupil moving and preventing morbid matter from fixing the iris and lens. A dark stable or a light bandage suspended orer the face is comforting to the patient and should not be forgotten. If the cause has been an injury from a blow with a whip or a bough, in going through a hedge, a mark will generally be left, and then it is, when inflammation has subsided, that zinci sulph. gr. iv. ad $z_{j}$. aquæ will prove useful in reducing if not altogether removing the nebula, as the more or less opaque spot is called. This will be seen to become blue round the edges, and gradually disappear till only a small blemish, technically known as an albugo, remains. It was the practice in former days to treat opacities of the cornea with caustic remedies, blowing powdered sugar through a quill on to the surface of the eye, and applying solid nitrate of silver, but such heroic remedies have proved to be quite unnecessary, and absorption can be facilitated by no greater excitants than the one above named, or alum. gr. iv., aq. $\bar{z} \mathrm{j}$.

Professor Williams recommends, in the inflammatory stage, the smearing of ext. belladonnæ ${ }^{1}$ upon the lids; this is rather crude pharmacy, and we should prefer his alternative sugges. tion :-Atropinæ sulph. gr. iv. ad zj j. aq. dest.

1 We have elsewhere apologised for some of the inelegant preparations in use among veterinary surgeons, and would call the pharmacist's 
Inflammation of the eyes may be of a more serious nature and of constitutional tendency and recurrence. Veterinarians of experience can distinguish between the two and treat the latter with aperients and febrifuges. In this disease the internal structures of the eye are involved and serious alterations follow. Repeated inflammations end in blindness, and too much importance cannot be attached to the examination of the eyes of a horse before making a purchase. If one eye is smaller than the other or looks puckered or three-cornered, there is something wrong. Although experienced veterinarians sometimes fail to detect these things, we think there is no guinea better spent by the amateur horseman than that paid for an examination as to soundness.

Torn Eyelids are generally caused by the reprehensible practice of leaving nails in stable walls, though horses have

attention to the field for improrement still open. The following recipe appeared (ISgo) in the Veterinary coiumn of a well-known periodical :-

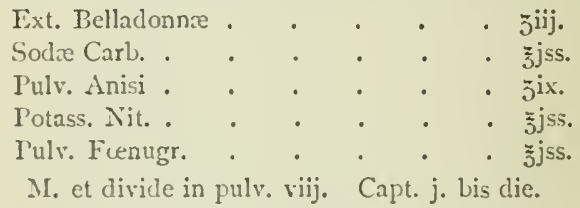

This is nothing to some of the messes prescribed by old writers who recommended the mixing of 'chamber lye,' \&c., and unblushingly suggested 'chamber pots' as suitable vessels for their manufacture.

Elegant pharmacy is now not unknown among veterinary practitioners, but they :ely very largely upon wholesale firms who make a speciality of supplying packeted drugs, standardised solutions for hypodermic injection, tabloids for pocket cases, \&.c. Like their medical confrères they hate dispensing, and rarely do it decently. Many would be glad to make arrangements with pharmacists, but there is a want of confidence, a suspicion of grod faith which has survived from the old farrier days, when vets. were not highly erlucated as they are now. There is room for mutual profit and convenience as between the V.S. and the dispensing chemist. The 'superior'smile of the pharmacist, when an inelegant formula is brought in, is not calculated to establish that good relationship which there should be, 
been known to tear their own eyes by rubbing against rough places. The edges need to be carefully brought together by means of fine sutures, as it is most important that the union should be quite level. Inflammation should be kept down with the soothing lotion before mentioned, and the wound, if possible, dressed with carbolised oil I in 40, care being taken to keep it from running into the eye.

Painting the sutured edges with equal parts of tinct. benz. co. and glycerin is good treatment. A shade so arranged as not to stick to or press upon the parts should be provided.

Grape, or Staphyloma, is rarely met with as a disease of the eye in horses, but occasionally occurs in cattle and frequently in dogs, especially of the pug and other large and prominent-eyed varieties, as Japanese toys, Blenheim and King Charles spaniels, \&c. It consists of a more or less hard whitish tumour upon the front of the eye, and is often the result of distemper, though it may occur at any time. It must be removed either by the knife or by caustics, the pain of which can be greatly mitigated by previous application of cocaine. The best plan perhaps is to secure a hold upon the tumour by passing a needle and stout thread through it and cutting off the greater part, finishing the work with several applications of argent. nit. Dogs that have been much reduced by distemper, or debilitated bystarvation or other causes, get a form of staphyloma in the centre of which is an ulcer, becoming larger if not treated, until eventually the aqueous humour runs out and blindness results. Ulcers of this kind are not so formidable as might be supposed (we are referring to dogs), and they can generally be rapidly mended by the application of

$$
\begin{aligned}
& \text { Argent. Nit. . . . . . gr. iv. } \\
& \text { Aq. Dest. . . . . . } \frac{3 j}{3} \text { M. }
\end{aligned}
$$

Large ragged ulcers on the cornea of the dog's eye will heal up and often clear up so well as to leave less blemish than does the flick of a whip upon the eye of the horse. Tonics should 
be given, and among the best for this purpose may be counted the following :-

Ferri Carb. Sacch. $\quad$ : $\quad$ : $\quad$ - gr. ij.
grininæ Sulph.

I. ft. pulv. Quotidie cum cibs.

This is for a terrier or small dog. Twice as much may be given to a spaniel, retriever, or setter; and three times the quantity to very large dogs, as mastiffs, St. Bernards, great Danes, \&c.

Diseases of the Humours of the Eye result from blows and often end in blindness by reason of lymph being deposited and organised. It is, however, impossible to say in a recent injury what the result will be, as the front chamber may be filled with blood-like matter and yet clear up again in a few weeks. Fomentations and treatment as for inflammation should be adopted and not despaired of while the colour of the contents shows changes. Blindness from concussion also happens, and from the rupture of minute vessels in the posterior chamber, and these cases also frequently recover.

The vitreous humour occupying the larger or posterior chamber of the eye undergoes changes in old dogs, becoming less transparent, and this is quite beyond remedy. Its progress is generally slow.

Since the discovery has been made that iodide of potassium may be given for a considerable time without constitutional injury, it is worth while prescribing for these lymph deposits in the deeper structures of the eye. $\Lambda$ grain or two daily for several weeks is quite a safe dose to prescribe, and we have scen great benefit accrue.

Cataract is an opacity of the crystalline lens. It may affect the capsule of the lens, the lens itself, or both, and is called respectively capsular, enticular, and capsulo-lenticular. In domesticated animals its treatment is not attempted. To the lay mind any opacity of the eye is a cataract, and pres. 
scribers get the credit of curing it when they have done no more than excite absorption of a cloud on the cornea.

A cataract can be distinguished by what is termed the 'catoptric test,' which consists in placing the animal in a dark place and passing a lighted candle or match from side to side, a little distance in front of the eye. In the normal eye thrce images of the light should be seen-two upright, and moving in the same direction as the candle, and the other inverted, and moving in the opposite. Depending upon the extent of a cataract, one or other of these images will be indistinct or absent.

Dislocation of the Eyeball caused by a fight or other injury is a ghastly spectacle, but there are not wanting trustworthy records of successful replacement. If it is attempted, no time must be lost. An antiseptic, as formalin or chinosol, being used to wash the injured structures and the operator's fingers. The outer angle of the eyelids should be divided and the globe pushed into place by pressure of the fingers. A compress should then be made of folded lint dressed with a lotion of

$$
\begin{aligned}
& \text { Liq. Plumbi • • • . • } 3^{\mathrm{ij} .} \\
& \text { Ol. Amygd. Dulc. ad • • • • 亏̌iv. M. }
\end{aligned}
$$

This accident to the eyeball is not so rare among pugs and other prominent-eyed dogs as one not accustomed to dog practice would imagine.

If the eye is lacerated or it cannot be replaced, it should be removed and the 'aching void ' treated with soothing remedies -that above mentioned is suitable.

Overflow of Tears is a matter upon which veterinarians are sometimes consulted. It is generally due to blocking up or obliteration of the tear duct. The face, whether of horse or dog, soon becomes scalded. If blocked by inflammation or matter, its function may be restored by reducing the inflammatory action and removing mechanical impediments. Many King Charles spaniels and dogs of that clạs are habitually 
tear-stained; their large limpid eyes appear to secrete more tears, and their flattened noses prevent the flow of them into the nose, as compared with other dogs. They are creatures of man's fancy, being bred from parents selected for the shortest noses, besides which it is an open secret that dog-fanciers compress their noses in puppyhood.

If the cause cannot be removed, the face should be dressed with ung. simplex or ung. lanolini. If greasy applications are objected to by the fair owners, a glycerin lotion not stronger than $I$ in 20 will prove grateful. 


\section{IVOUNDS, SORES, ETC, IN ANIMALS}

The Treatment of Wounds is a large subject, and many books have been written upon it, but there are a few simple rules to be observed which are applicable to all animals, including man. Without entering into an elaborate discussion on germ theories, we may say that in nothing has the healing art advanced so much during the Victorian era as in surgery. It must be admitted that veterinary practitioners have generally a better claim to the title surgeon than have the majority of those by whom the unqualified title is borne. The ever-increasing number of specialists in the medical profession, and the facilities for getting operations done at hospitals, have benefited the public, but not the men who call themselves surgeons, and who practise for years without ever venturing on an important operation. How many college surgeons, for example, ever performed such an operation as ovariotomy? Hundreds of country vets. perform it daily, and under the most disadvantageous conditions. The animals on which they operate are generally smaller, and consequently more difficult to deal with. It is true that the human patient is more prone to blood-poisoning and other bad sequelie than animals whose habits are simpler; but against this may be reckoned the advantage the ordinary surgeon has in the fact that his patient is more amenable to control. The animal doctor has to allow for the chance of his splints and bandages being bitten off, and himself bitten, kicked, or crushed while attempting to help his patient.

The principal varieties of wounds to be dealt with may be classified as incised, punctured, lacerated, and contused ; and to these may be added gunshot and poisoned wounds. 
Incised Wounds are those in which a clean cut is made with a sharp instrument, dividing the structure evenly without tearing or stretching the tissues-a clean division in short. The disposition of such a wound to gape open or not will depend upon the direction it takes with regard to the muscular fibres beneath, supposing it to be where muscles are found. This definition, though it applies well enough to men, is scarcely complete in reference to animals. There are many wounds received by horses below the knees and hocks, where no muscles exist, and a small wound may be of vastly more importance than a large one upon a fleshy or muscular part of the body. According to the nature of the structures injured must the treatment be regulated. In the case of an ordinary incised wound in the fleshy parts of the body or limbs, the treatment should be directed to bringing the edges in apposition with the least possible delay, pouring on an antiseptic dressing even before sutures or strapping are ready for application. An immediate dressing of acid. carbolic. I part, ol. olivæ 40, has the most agreeable effect in relieving pain, and will frequently cause cuts about the hand of man or the limbs of horses to heal by first intention, or adhesive inflammation as it is called. If the wound be a large one, it will take the 'sting' out, and make the animal more amenable.

Plaster does not answer very well with animals, on account of their hairy surfaces and the ease with which they can bite or lick it off. One or more sutures need to be put in to secure a wound of any size; these should not be drawn too tight, as there is always swelling afterwards, and the stitches may be torn out. In wounds of sufficient magnitude to require stitches, a certain amount of discharge may be looked for, and the lower end should not be brought together quite so closely as the remainder, so as to allow of a drain. It is a very important consideration to allow of drainage, as pocket wounds sometimes form sinuses or pipes, and need prolonged treatinent.

It is sometimes necessary in punctured wounds to cnlarge the orifice, always in a downward direction, in order to allow of 
free drainage. Splinters and other foreign bodies are more apt to be present in wounds of this class than simple incised or even lacerated wounds. Splinters of wood, iron, \&c., sometimes re. main in the flesh for a long time, and small ones especially find their way out in the most unexpected places, nails, wire, and needles making the most extraordinary travels.

Tetanus, or lock-jaw as it is more commonly called, follows more frequently upon punctured wounds than others. The magnitude of the wound is no measure of the tendency to tetanus. It more often results from a prick in shoeing than from a great wound inflicted by machinery or collision.

An explanation is found in a specific bacillus being the cause of tetanus. The hands and feet being more in contact with the soil or substances in which the organism is found.

In some tropical swamps it is so common that tetanus carries off a large proportion of persons and animals suffering from wounds.

Perfect asepsis renders its invasion impossible, and it is now quite rare in hospital practice, where formerly it was most dreaded.

Lacerated Wounds may be of any shape and size, but differ from the former varieties in the skin being torn and ragged, and often the deeper structures besides. To bring the injured parts into apposition is the object to be aimed at, whether by sutures, bandages, plaster, or other contrivance. To the inexperienced surgeon the bleeding is a difficulty, but it is very rarely dangerous even when large vessels are divided, for the animal will have either bled to death before his services can be obtained, or the vessels will have plugged themselves. If an artery can be seen spurting bright red blood, it should be secured by a ligature, and the ends left long to hang out of the wound for removal on a subsequent occasion. Liq. ferri perchlor. and other styptics may be used, but hæmorrhage may generally be easily enough arrested by pressure, according to the situation of the wound. The operator must keep cool, and consider where and how the pressure should be 
applied. It should be recollected that there is a natural tendency or effort on the part of nature in the rapid formation of clots ; vessels may be seen sometimes with clots a foot long hanging down like a rough cord, and effectually plugging the cut end till others shall have taken up the work, and allowed the divided or lost artery to waste away.

The loss of a large vessel even may soon be compensated by the additional work undertaken by others-as, for instance, when a jugular vein is rendered impervious by inflammatory action, the circulation in the head is carried on as well, or nearly so, as before the accident.

Unless a foreign body is suspected to be present in a wound, it is generally best to bind it up in the blood immediately, and not to foment or remove clots unless it is very thoroughly done with modern antiseptics. It is to be hoped that the practice of bathing a fresh clean cut with hot water till it has the appearance of butcher's meat has pretty well gone out of datc.

The different domestic animals vary very considerably in their power of recovering from wounds.

Horses are perhaps the most liable to tetanus and dogs the least, horned stock coming between them. There is no record of tetanus in the cat, so far as we are aware.

Wounds in dogs heal more often by simple adhesion than is the case with any other animal. Such severe operations as ovariotomy, necessitating a division of the flank and the introduction of the finger into the abdomen, will often heal without the formation of matter.

The after-treatment of wounds needs some experience, but the chief aim should be to excite healthy granulations, and the discharge of 'laudable pus,' to quote a favourite expression from Professor Simonds. The efforts of nature to repair wounds sometimes require stimulating and at others repressing; hence it is the duty of the surgeon where a wound is pale in colour, with a thin reddish or frothy matter, to stimulate with ung. resine or ol. tereb. rub. I pt., ol. sesamx 3 pts., applying daily till red healthy granulations begin to show, and thicker, yellower matter to appear. 'Proud flesh,' as it is 
called, is but the too rapid or excessive production of granulations, and a large wound may at first require remedies to produce them, and, later on, others to keep them in check ; for the latter purpose nothing can compare with zinci chlorid. or cupri suiph. For small surfaces argent. nit. answers well enough, and forms a dry scab which may be picked off roughly from time to time, thereby bringing the edges of the skin together, and leaving less blemish than if left to nature, even supposing the granulations did not grow out, and form a soft, painful surface, blecding upon the least touch. (See Broken Knees, p. ${ }^{6}$.)

The principles of treatment of a wound may be summed up as follows :-(1) Arrest hæmorrhage; (2) remove foreign bodies, and dress antiseptically; (3) bring the edges into apposition ; (4) and again apply an antiseptic dressing.

Professor Pritchard and others have called attention to the fact that, where sutures are retained for a day or two, the wound will eventually heal opposite the marks made by the needle, no matter how long the process of union may take.

Abscesses sometimes result from wounds, but more frequently from severe bruises. They niay be briefly described as of two kinds, serous and phlegmonous.

Serous abscesses occur in all domesticated animals, but most frequently in the dog and cat, as the result of injuries received in combat. They occur, too, in those parts where both dogs and cats usually grapple one another-namely, on the throat, ears, and face. Their formation is very rapid, producing great swelling and much inconvenience, but nothing like the pain caused by abscesses containing pus. The inner surface of the ear is frequently the seat of serous abscess, and must there be very painful. It is perfectly useless to attempt any treatment until the knife has been freely used. The abscess must be boldly slit up, and the fluid, which is of a reddishyellow colour, evacuated. Nor is this all that is necessary, for it will be found as full as ever again next day, and the lips of the wound sealed up, unless some 'digestive' remedy has been applied to destroy the serous sac or lining menibrane of 
the abscess which secretes the fluid. A seton dressed with some irritant, such as ol. tereb., or hyd. bichlor., gr. v., sp. vini $5 \mathrm{j}$., does well in some cases. The ends of the tape or string composing the seton should be knotted, and not tied together, as both dogs and cats have a happy knack of striking the ear and face with the hind leg, and if the foot hangs up in a tied seton it causes great and unnecessary pain, and perhaps irreparable injury. With horses and cattle there is not the same danger; when pus begins to form, the seton may be removed, and nothing more is needed than a little healing ointment, such as ung. resinæ and cer. cetacei or ung. zinci ox. $\bar{\jmath} \mathrm{j}$., with acid. carbolic. $m_{\mathrm{x}}$.

Phlegmonous or pus abscess is more painful, and usually longer in forming than the serous, though arising from much the same causes. It must not be lanced until ripe, but the process may be hastened by hot fomentation or the application of lin. camph. co. or other warm embrocation. If left alone abscesses usually (though not always) 'point' - that is to say, one part becomes more prominent, and when felt is found to be much thinner, eventually breaking upon some motion of the patient, or blow from one of its kind. The surgeon may save the animal a good deal of suffering, by boldly lancing at the 'point' or thinnest part, and squeezing out the matter, which is often very thick, and sometimes granular if long neglected. A pledget of tow dipped in ol. tereb. rub. or lin. alb. or ung. resinx, will facilitate the process of evacuation, or if the situation is convenient, and the patient under control, an injection of either of the fluid remedies suggested, by means of a metal syringe, ' will answer better still. It is difficult to get a pledget of ung. resinæ to stop in, even if the patient is quiet.

\section{Ulcers occupy so considerable a space in old works on}

We are aware of the chemical objection to using a metal syringe with hyd. lichlor., lut think the destruction of a fourpenny syringe the lesser cvil. Glass syringes are very lialole to have the nozzles broken by the sti.klen movements of the patient. A rulcanite one may, of course, be used. 
the healing art that it may be assumed that they were more common than now-famine and dirt have always contributed to the production of ulcers, and from these causes they are still to be found more frequently among savage races than in civilised communities.

Ulcers among domesticated animals in the British Islands are not of frequent occurrence, if we except the specific ulcers of certain affections, as foot-and-mouth disease, or loss of structure following upon injuries such as sinus-ulcers-quittor, poll-evil, fistulous withers, \&c.; but of ulcers breaking out upon the body as a blood-disease, we do not see many examples in these times of better stabling and sanitation. Where, however, an animal shows a disposition to form ulcers without any external or exciting cause, it may be fairly assumed that the blood wants 'mending or sweetening' as some old works state it. Alteratives and tonics, such as are prescribed elsewhere in this work, mineral tonics especially, and good diet are to be recommended. Ulcers may be divided into weak, indolent, inflamed, gangrenous, \&c., but they all need treating with severity, hence the black oils and 'sublimate' treatment that is the practical result of the experience of ages. No doubt a careful distinction between the ulcers is an advantage to doctor and patient, but the chief thing to be arrived at is to destroy the unhealthy sore, and produce in its place a healthy wound which will heal up by the ordinary process of granulation. This may be done by the hot iron, or dusting it over with hyd. bichlor. or alum. exsicc. or alum. exsicc. with various proportions of flour, or zinci ox. vel carb. with pulv. amyli, or dressings of cupri sulph. vel acetas, zinci sulph. or zinci chlor. Each and all of these have proved effectual, besides a score of other remedies which might be named, but we most strongly recommend alum. exsicc. When applied for such a purpose this article should be rubbed quite smooth, and may be disguised by preparing it secundum artem with pulv. bol. armen. vel p. curcuma vel carbo animalis (carbo lig. is inconveniently light).

If a liquid application is preferred, alum. sulph. I in 20 , or 
cupri sulph. saturated solution, or hyd. bichlor. gr. x. ad $5 \mathbf{j}$. of S.V.M., may be prescribed. Washing ulcers clean with warm water and soap, and dusting on the dry porders above sug. gested, is however, we consider, preferable treatment. Pressure is also helpful, especially if the situation lends itself to such treatment by bandaging.

Ulcers in the mouth, upon the tongue, and about the muzzle, are frequent in cattle and sheep as a result of foot-andmouth disease, which see.

For all kinds of ulcers alum is a specific. The most rapid, and, to those who have not tried it, the most surprising results follow the sponging of the ulcers with an aqueous solution of alum. Two or three days will often suffice to completely heal large ragged surfaces inside the mouth; but the process is not so rapid outside, as it is not assisted by the saliva, and is more or less checked by contact with foreign bodies and exposure to the atmosphere.

Ulccrs of the hind legs occur in round-legged, coarse-bred animals with a tendency to farcy, and should be treated with

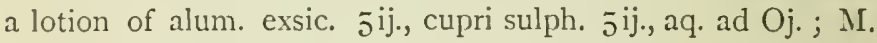
ft. lotio. For internal remedies, see Farcy, p. I I5.

Sinus-Ulcers or Fistula are of much importance to the veterinarian. They may and do occur in all sorts of places, but we can here only consider a few of the more important, as quittor, poll-cvil, and fistulous wither.

Quittor is a sinus-ulcer having its orifice between hair and hoof, usually on the inside quarter of the horse's foot.

It may arise from a neglected prick or festered corn, or tread from the opposite foot. The orifice may be very small, and the owner think lightly of it; but he will soon find, to his cost, that another pipe or sinus has formed in front of the old one, and the reterinary surgeon when called in may find that l)ranches have already begun to grow. I'ipes rumning in all directions are found in the foot when an incurable case is dissected. 'The treatment aims at destroying these pipes. 
In a few mild cases the exit of the matter is followed by speedy recovery, but in the majority of cases the opening shows no tendency to heal, the discharge continues, and the pain and lameness remain. This is due to one of two causes : either there is some dead portion of tissue lodged in the part, as a piece of decaying bone or fascia, or else the wall of the sinus has become so thick and hard as to resist the ordinary reparative power of nature.

Whicherer of these be the case, the part, if not interfered with, would be a considerable time before it resumed its normal condition. In case of the lodgment of a piece of dead bone, nature would doubtless, in time, remore it by the constant suppuration; but time and suffering are both saved by interference. Both of the perpetuating causes may frequently be removed by the same means: viz., setting up such an amount of inflammation as shall destroy the callous lining membrane, and thoroughly detach any portion of dead tissuc, which would then be removed in the discharge, and a healthy granulating surface left. To this end numerous means are employed. Blisters and firing, or even incision, are resorted to, but the two latter require a qualified professional man. Blisters are of little use, save in conjunction with caustic injections. The old farriers used corrosive sublimate in powder. It was placed in a small hollow cylinder of paper, and pushed into the sinus. This method is very good when only one passage exists, but too often the sinus has three or four collateral branches, in which the plug of caustic would not produce its effects. The sublimate is a valuable agent, but it should be used in solution and forcibly injected by means of a syringe, so as to come in contact with the whole of the sinus.

A good form of injection is-

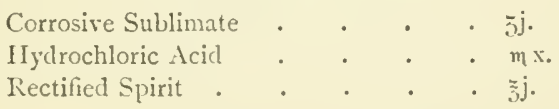

This solution may be uscd once a day for two days, and then either diluted to half the strength, or used once every 
three days. If there is still much pain after a fortnight of this treatment, apply a smart blister. Solutions of the sulphates of zinc, iron, and copper are sometimes used, or a mixture of all three under the name of Vallate's solution, which has proved successful sometimes where all other agents have failed. The general health must not be neglected. An occasional dose of physic is useful, and at the outset a dose or two of opium to allay the great pain, or belladonna extract or sol. of cocaine.

The old school of practitioners were certainly very successful in the treatment of quittors and poll-evil, and had a great deal more practice with the latter than we have since stables are not now built low and dark, and blows on the head, accidental or otherwise, are not so frequently received.

The old method was to secure the animal with a twitch or by holding up the opposite foot, or in some cases casting the animal with ropes and then ascertaining the direction of the 'pipes' by means of a probe; this done, the 'sublimate' would be rolled in thin paper, and pushed down as far as it would go. Some practitioners poultice, enveloping the whole foot for several days, during which the animal will manifest much pain until a slough or 'core' comes out. Then, if the sinus were truly 'bottomed,' a healthy discharge of pus would follow, and the wound would eventually heal. Too often, however, there is permanent injury to the coronary band, and a false quarter or sand-crack is the result.

A quittor then is alway's a very scrious matter, and often ends in sending for the knacker, after a great deal of expense and trouble has been incurred.

One celebrated man effected a great many cures in cases that had been regarded as hopeless by using stick zinci chlor. His plan was to push the stick of zinc in and out of the sinus nearly every day, and the zine being easily lipuefied would be to a certain extent pumped into the branched sinuses - he also had the sole, crust, bars, and all cut down to the quick, and poulticed for a few days.

The modern treatment is to explore the sinus with probes and lay the fipes open by means of a bistoury and director, in- 
jecting some caustic solution if necessary with a syringe made on purpose, and having two eyes or finger-holes on the barrel so as to enable the operator to do it with one hand. Carbolic acid has been used successfully, and other agents have effected the destruction of the sinuses, which, as we remarked at the opening of the chapter, is the object of whatever system of treatment is adopted.

In ail cases of quittor the shoe should first be remored, and if arising from a corn or prick these should be well pared out, and drainage got from below by poultices.

Poll-Evil is a sinus, or more frequently a number of sinuses, situated in that part of the horse called the poll-the back of the head or top of the neck. It is caused by blows or other injuries, and was much more frequently met with in times past when stables were low and dark. Now it is almost confined to mines and underground works. The treatment required is the same as for quittor (as explained in the preceding section), namely, the destruction of the sinuses and the production of a healthy wound in the place of the destructive pipes or sinuses. In some respects poll-evil is more easily treated than quittor, inasmuch as a seton can be put through the part, and a dependent orifice thereby obtained. The same agents are used as for quittor, and, despite warnings about destroying the ligament (nuchæ) which supports the head, and the danger of coming down on the spinal cord between the two first bones of the neck, it generally proves successful. Injections of zinci chlor., cupri sulph., \&c., often answer well enough, but for a case of any standing we advise a coarse seton dressed in zinci chlor. at first, and afterwards with ol. terebinth. When once the sinus is destroyed the wound becomes healthy, and heals up with the ordinary treatment accorded to granulating wounds. (See Wounds.)

Fistula.-Fistulous wither is another variety of piped wound, and is caused by hard saddle-trees or harness pinching and crushing the wither. It never occurs in a well-managed stable, as it is simply a matter of looking after the saddles 
and seeing that they are properly stuffed or renewed from time to time. When fistula is once established the pipe must be traced with a probe, slit open with a knife, or destroyed by a seton, taking care to bring out the lower end of the tape as low down as possible, so as to obtain a good drain from the wound. All this may be prevented if, upon discovery that the withers are wrung, and the 'galled jade' winces at the prospect of saddling, a good fomentation of hot water with an ounce of glycerin to the quart of water be used, and finally an evaporating lotion as-

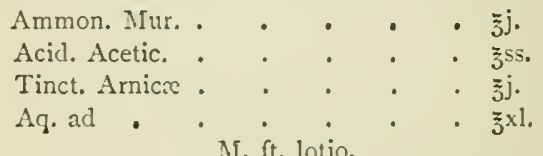

To be applied two or three times a day, allowing the parts to dry without clothing.

Saddle and Collar Galls are frequently produced by sudden chills in taking off saddles and collars while the horse is hot; good grooms unbuckle and ease them while they are attending to other matters, and so allow the parts to cool slowly.

Bad-fitting collars, especially if too large, are a frequent cause of galls, and useful horses are incapacitated for work by wounds no larger than a sixpence. This is galling both to the animal and the owner, and a lotion that will quickly mend it is in demand. There is a lotion much used by carters, and with some measure of success, which we can hardly recommend here. It is always of the right temperature for application, and has a varying amount of saline material in it, depending a good deal upon the drinking habits of the carter. We should recommend, as a well-proved remedy, the following :-

\begin{tabular}{|c|c|c|c|c|c|}
\hline Acid. Sulph. & & & & & \\
\hline Alum. Sulph. & - & & 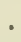 & & \\
\hline Cupri Sulph. & ${ }^{\circ}$ & & ${ }^{\circ}$ & & - \\
\hline l'otass, Nit. & 4 & $\theta^{\circ}$ & • & • & . \\
\hline Aque ad . & - & . & - & - & - \\
\hline
\end{tabular}

M. ft. lotio. Shalic before applying. 
Frequently applied and allowed to dry on, this lotion has the effect of quickly drying up a sore shoulder or saddle-gall, and appears to harden and thicken the skin to resist future rubs. The practitioner should, if possible, examine the collars, pads, sc., to see if they will discredit his medicaments by producing fresh galls as soon as the patient is put to work.

Plumbi acetas is a time-honoured and helpful agent, but we recommend the above to the counter prescriber for more reasons than one.

Sitfasts are irregular-shaped pieces of skin in the process of separation from the body, and may be compared to hard corns on the human hand or foot. 'They become thickened and insensible in the middle, and press upon the surrounding soft tissues like a foreign body, such as a stone tied on to a gall. They are caused by the repeated bruising of badly fitting harness, and are most frequently found on the withers and under the collar. Their presence causes a great deal of pain, and recovery is impossible while the cause remains. Poulticing and fomentation facilitate the separation of the slough or dead skin, and where poultices cannot be applied an ointment such as the following will answer best :-

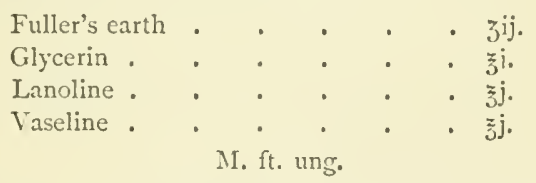

The whole sore should be enveloped in this ointment, which has the effect of promoting disintegration of the dead tissue while keeping soft and healthy the surrounding wound. Some of the old authors-Percivall for instance-advised repeated mild blisters, but Professor Williams, in his work on Veterinary Surgery, recommends the knife in preference. Sitfasts, as the name implies, do, indeed, sit fast, and something more than a powerful thumb-nail is needed to remove them in many cases. Once removed, the treatment needed 
is that usually accorded to granulating wounds. (See Wounds, p. 270 .)

\section{Sore Shoulders, \&c.-Badly fitting harness frequently} causes sores on the skin, the shoulders and back suffering most. Such injuries vary from mere loss of hair to deep angry wounds; but something between these two extremes is what advice is generally sought for. Should the skin be not broken, but present a tender surface, a good application can be obtained by heating together an ounce of glycerin and two drachnus of fuller's earth; to be smeared on cold. A simple skin wound will yield readily to salt and water, which may be increased in apparent value if coloured with tinct. lavender. But sores caused by harness are too frequently of a more serious character. Careless horsekeepers neglect a slight wound, and the irritation being kept up, a deep circular sore is the result, the margins of which become callous and the centre covered by a hard scab, under which is always more or less matter. Such a wound, commonly called from the centre scab a sitfast, is most obstinate, showing little tendency to heal un. aided. No half measures are of use; the scab must be removed, and the whole sore dressed with some caustic, as nitrate of silver or even corrosive sublimate (the first in stick, the latter dissolved in spirit of wine, $\bar{j} \mathrm{j}$. to the ounce). A very bad case is radically cured by an incision right through it, so as to alter the circular to an elliptical wound, at the same time starting healthy granulations.

It is common amongst heavy horses to see on the inside of saddles or collars the padding roughly cut out over a wound with a view to prevent pressure upon it. This certainly spoils the harness, and very seldom answers, as the fadding protrudes and keeps irritating the wound. The neatest, most effectual and economical method of relieving any sore from pressure, is to apply pads to the harness so that one rests above the sore and one below it. 'The pads may be made of chamois-leather and stuffed with curled horsehair. Common-sense will point out that a pad must not be so applied to a collar as to press on the jugular veins. 
Open Joints.-A special difficulty in treating these cases is to check the flow of joint oil or synovia. 'To do this it is first of all necessary to fix the joint, so as to prevent movement. Some joints, as the fetlock, \&c., are easily fixed by a bandage ; larger joints, as the knee and hock, usually need some contrivance, as an iron bar or piece of wood, put on to the leg like a splint. The higher joints cannot be fixed in this manner, but if the lower ones be fixed, motion is always more or less limited in the one immediately above.

In the case of the stifle joint, from the anatomical arrangement of certain tendons, movement cannot take place if the hock be fixed. The jaw-joint is a very awkward one to fix, as by doing so mastication is stopped; but this must be done for a time, and motion must be limited till recovery ensues. A tight nose-band effectually does this. By some, blisters are applied round the opening and over the joint ; they act very well ; they increase the granulating process, and keep the joint still.

In ail such cases, however, it is an advantage to coagulate the discharge. The discharge is albuminous, so that there are a number of substances to use. A common one is alum; not by itself, but as an ingredient of what is called OPEN-JOINT POWDER, made thus--

$$
\begin{aligned}
& \text { Alum . } \\
& \text { Ferri Sulph. } \\
& \text { Pulv. Myrrhæ } \\
& \text { Finely powder, and sprinkle on to the part. }
\end{aligned}
$$

The objection to this is, that albumen is redissolved by alum in excess. The two best and neatest applications are nitrate of silver in the solid form, and corrosive sublimate ( $3 \mathrm{j}$. in 亏j. S.V.R.) applied with a feather. Care must be taken with both of these substances not to introduce them into the joint, but merely to touch the escaping synovia at the opening. Finally, never remove the plug of coagulated synovia from the opening when dressing a case. 
'Grease' in Horses.-This expressive name is given to a skin disease affecting the legs. Heavy cart-horses are most subject to it. The hind legs seem more prone to become 'greasy' than the fore. Grease varies in degree, from a small spot covered with short broken hairs, representing merely a wet surface, to a state in which the leg up to near the hock is covered with red, painful granulations and a stinking discharge. The treatment of this disease is generally tedious, and often unsuccessful ; this is probably owing to neglect in dressing as much as to the natural obstinacy of the affection. The restiveness of the animal, coupled with the offensive smell arising from a greasy leg, renders the duty of dressing so disagreeable as to ensure neglect by all but the most patient of men. A dressing should be astringent, caustic, and disinfectant. These qualities are combined in the following :-

$\left.\begin{array}{llll}\text { Ferri Sulph. } & \cdot & \cdot & \cdot \\ \text { Zinci Sulph. } & \cdot & \cdot & \cdot \\ \text { Cupri Sulph. } & \cdot & \cdot & .\end{array}\right\}$ of each I lb.

Dissolve in a gallon of boiling water and add Carbolic Acid $4 \mathrm{oz}$.

The sulphates of copper and zinc are also given internally in drachm doses, their action upon the lymphatics of the lcg being unquestionable. Three or four balls per week of the above strength may be given.

If there is much swelling of the affected limbs an aloetic ball may be given; the animal should be kept at work save during the first two or three dressings, as then in bad cases the pain is considerable. As a last resort, firing may be had recourse to ; but as this entails at least two months' idleness, only a valuable horse is worth it.

Fractures are called simple when the bone is only snapped in two, comminuted when it is splintered, and compound if a wound of the skin accompanics and comnunicates with the fracture. The bones most frequently broken are the long lones of the limbs. Fractures of ribs or skull are dangerous from the chance of injury to organs within, and should only 
be treated by an expert. We therefore confine our remarks to more simple cases.

Detection of a fracture below the knee or hock is an easy matter by the obvious deformity; above this, however, where the bone is well covered with flesh, a careful examination is often necessary.

The symptoms of fracture, in addition to pain and lameness, with sometimes swelling and displacement, are twitching of the muscles and crepitus, i.e. a grating sound heard on moving the bone so as to bring the broken ends into contact. The twitching of the muscles is due to irritation caused by the broken ends. A fracture may be mistaken for dislocation, and vice vers $\hat{x}$. It may therefore be remembered that ordinary extension will reduce a fracture to its proper position, while considerable force is required to reduce a dislocation, and when reduced it remains fixed. A fracture, say of the arm of a dog, is accompanied by swelling, due to blood effused at the part from lacerated vessels. This is rapidly absorbed, and in its place we find organisable lymph, which gradually becomes condensed. This material not only surrounds the fracture, but is found between the ends of the bone.

This change takes place in about a week. Then we have bony deposit between and around the ends of the bone, which in time replaces all the plastic material first formed, acting like a ferule to the part. This bony ring in surgery is known as the Provisional Callus, in contradistinction to the Definitive Callus, i.e the portion between the ends of the bone. These are not separate, but continuous; the difference is that the superfluous encircling mass becomes absorbed, while the intermediate portion remains as the union. Really, however, this also changes-for at first it is a solid, compact mass, but in time, by absorption, it assumes the form and structure of the original bone.

The treatment of fracture, then, consists of two parts - setting and retaining the ends of the bone. Correct adaptation is necessary, as Nature attempts repair in whatever position the parts are kept, and so might perpetuate deformity. Success is 
shown by the outline of the limb and by the grating together of the ends of the bone. To retain them in position in the best manner is done by applying such an apparatus as will take the form of the parts, and prevent movement without injuring any of the tissues. We may use either 'splints,' as thin pieces of wood, cardboard, or gutta-percha, or, better than either perhaps, a long bandage saturated with glue or starch, to give it the requisite firmness. If the bandage be strengthened with glue, this should be melted with a little glycerin instead of water. It is thus made softer and tougher.

Do not apply the starched bandage directly to the limb. First roll a dry bandage carefully round, beginning at the toes, and applying it as high on the limb as possible. The joint above and below a fracture should always be fixed if practicable, as movement is thereby reduced to a minimum. A bandage should never be applied to a limb so as to leave a distal portion uncovered, for the compression above is certain to cause swelling below and, if continued, congestion or even gangrene. No matter what part of a limb is fractured, if a bandage be applied, commence at the toes, so as to have a uniform pressure. Over the dry bandage apply the starched or glued one. Secure the ends, and keep the dog in a small cage, so as to limit his movements.

This apparatus should remain on for about fourteen days, and be examined daily to see it is not loose or too tight. A compound fracture had better be treated with splints and a simple bandage, so arranged as to allow the wound to be examined without remoring the whole arrangement. A comminuted fracture may be treated like a simple one ; but if also compound, and any pieces of bone are quite detached, they should be removed.

In fractures of such bones as the thigh, where we cannot apply a bandage, we can fix the hock and stifle joints, and so prevent a good deal of movement. A plaster over the part will also aid if the hair be previously cut short, otherwise it only causes annoyance. These fractures only pay for treatment in pet dogs or bitches, as frequently a false joint saves 
the animal's life, but renders him lame for life. These remarks specially refer to dogs, but are equally applicable to other animals. The bones of the lower animals unite with great rapidity; but the money value is generally the standard by which the advisability of treatment is judged. Those kept for stud purposes are generally worth a trial; those for the butcher never. 


\section{DISEASES OF POULTRY}

NEARLY all the diseases of poultry are the result of cold, wet, want of cleanliness, bad feeding, or overcrowding, and several are very infectious. The diseases in all classes of domestic fowls or game are very similar, so that the remarks made in regard to poultry apply generally to most of the birds included. It may be remarked that ducks are especially bad to manage when ill ; they seem to be very little susceptible to medicines, and when not feeding naturally, almost all that can be done is to 'cram' them.

\section{POULTRI}

Abortion.--Sometimes when hustled or violently driven about, hens suffer much in this way. The bird should be put away in a quiet place, and fed sparingly on soft food to which is added a small piece of prepared chalk, or a pinch of sodii bicarb. may be placed in the drinking water.

Apoplexy.-Birds, apparently in robust health, fall suddenly, and are found either dead or insensible. Hens may die on the nest while cjecting the egg. The only hope of cure is in immediate bleeding by opening one of the largest veins on the inner side of the wings by a longitudinal incision. So long as the thumb is pressed on the vein at any point between the body and the cut, blood will flow. The disease is generally caused by a too liberal or a too stimulating dict. Hempseed or an orer-supply of pea-or bean-meal may occasion it. If the fowl should recover after treatment, keep it very quict for a 
few days, and give it only soft food. Afterwards a pill should be given of

$$
\begin{aligned}
& \text { Pulv, Ext. Coloc. Co. • • • gr. x. } \\
& \text { Ilydrarg. Subchlor. . • . . gr. j. }
\end{aligned}
$$

The bird should have rest for a few days. If symptoms of paralysis should be observed, liq. strychninæ in 5-minim doses may be given twice a day in soft food or in the drinking-water.

Baldness and White Comb.-White Comb is a hard scurfy condition of the organ. Give good green food and exercise, and a 5-grain Plummer's pill erery night for a week. The parts should be dressed daily with sulphur or tar ointment. Instead of the pill a tablespoonful of castor-oil, followed by a teaspoonful of sulphur in the food, may be given daily for ten days. One 'authority' on the subject recommends an ointment as follows, and claims that it is a specific for the disease:

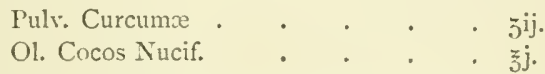

Black Rot.-This disease was more common formerly than now. Its symptoms were blackening of the comb and swelling of the legs and feet. The treatment consisted in giving $\bar{z}$ ss. castor oil, or calomel gr. ij., followed by a course of tonic treatment, such as 5 ss. doses of syr. ferri phosph. co. twice a day for a fortnight, with warm and nourishing food.

Bronchitis.-This may or may not be associated with ordinary catarrh. It is characterised by frequent coughing. Five grains pil. scillæ co. twice daily, and 'Douglass Mixture' in the water, is the best treatment. A grain or two of cayenne may be added to the food. The bird should be kept in dry, comfortable quarters.

Bumble Foot.-This term signifies deformity of the feet, caused, it is supposed, by pressure from the perch, especially in large heavy birds, provided with square instead of round bars.

No treatment is of avail. 
Canker or Ulceration.-This exhibits itselt as a cheesy growth or ulcerated condition of the mucous membranes of the mouth or throat, sometimes also affecting the eyes and nostrils so as to cause actual suffocation. Among the remedies recommended are liq. sodæ chlor. as a lotion with which to swab the mouth and throat, or carbolic lotion, $I$ in 20 , used in the same way. Some recommend the growth to be scraped away, and the spots touched with argent. nitr. As it is now generally believed that the disease is of a tuberculous nature and highly contagious, birds affected in this way should be promptly destroyed and their carcases burned.

Catarrh shows itself by a watery or adhesive discharge from the nostrils with swelling of the eyelids. When the discharge becomes purulent and offensive, and the other symptoms are aggravated, the disease is called Roup (which see). A dry warm situation and stimulating food will often relieve slight cases, which should never be neglected. At the first symptoms affected birds should be removed to comfortable, warm and dry quarters, and fed moderately on soft warm food to which have been added pulv. capsici, pulv. glycyrrhizæ, pulv. anisi, pulv. cocci, ferri sulph., āà partes aquales, sufficient to make the food piquant. Or I pil. scillæ co. may be given twice a day. The above powder will be found useful as a concentrated poultry spice, of which a teaspoonful daily in the food is sufficient for about fifteen to twenty birds.

Cholera.-Chicken cholera, although not so common as it formerly was, is still at times a severe scourge in the poultry yard. In character and symptoms it is not unlike human cholera, appearing in hot weather and sometimes becoming epidemic. It is due to a specific bacillus.

Its symptoms, although not always uniform, are a sudden accession of thirst, accompanicd by diarrhoea, at first of a greenish, then 'rice water' description. 'There is great weakness and prostration, with probably a 'cramped' condition.

No treatment is desirable, but removal and burning of in. fucted carcases and thorough disinfection of coops are essential. 
Cramp.-Young chickens sometimes suffer from cramp when exposed to damp and cold wind in early spring. When observed, they should at once be removed to a dry, clean, boarded floor, liberally sprinkled with sand or fine peat moss, and the legs rubbed two or three times a day with ' white oils.'

Good henwives bring in the chicks and cover with flannel, placing near a fire but not above it. Bottom heat from hot water bottles does not suit chickens, but they quickly respond to warmth from above.

Crop-bound.-This name is given to distension of the crop, either by over-feeding and subsequent swelling of the grain, or by the presence of some single object too large to pass into the stomach. To treat it, first pour warm water down the throat to soften and loosen the food, kneading the crop for some time with the fingers. Then give a tablespoonful of castor-oil or from 5 to 8 grains of jalap mixed in butter. If this should not be effective, as a last resort the crop must be opened. Cut into the upper part of the crop with a s'iarp penknife about an inch, loosen the mass with some blunt instrument, and remove it; if very offensive, wash the crop out with warm water. Feed for a few days on soft food, adding a little of the spice recommended under Catarrh. If the incision is small it may be left, if large a stitch or two should be inserted.

Crop, So!t or Swelled.-This is another form of distension of the crop as the result of indigestion. Treatment as for the first-mentioned form will be suitable, allowing only a moderate quantity of water and soft cooked food for a time. In both forms a comp. rhubarb pill is occasionally useful.

Croup and Canker.-A purge of castor-oil should be first given, after which the following pill will often be found useful :-

$$
\begin{aligned}
& \text { Pulv. Capsici . • . . gr. v. } \\
& \text { Pulv. Cupri Sulphat. . . . gr. iij. } \\
& \text { Syrup. . . . . q. s. }
\end{aligned}
$$

Fiat massa. Make into an oval pill, and roll in liquorice powder. A pill to be given twice a day. 
Debility.-Anything that tends to lower the vitality of the birds will induce this condition, apart from any actual disease. It is best treated by the exhibition of chemical food in 5 ss. doses, or the 'Douglass Mixture' as recommended below.

'Douglass Mixture' for Moulting Birds.-Dissolve I oz. of iron sulpliate in I quart of water, add I drachm of dilute sulphuric acid, and put $\mathrm{I}$ teasponful of this mixture in each quart of drinking water. When chickens droop and seem to suffer as the feathers on the head grow, give them once a day meat minced fine and canary-seed.

Diarihœa is common in changeable, cold, wet weather. Eating fish also causes it. If observed in its early stages it can usually be checked by feeding on boiled rice, with which is mixed a little chalk in fine powder, or a pill of the same to which has been added 5 minims spt. camph. may be given twice a day, and if very severe pulv. opii gr. $\frac{1}{2}$ may be given with it.

Dysentery (Bloody Flux).--Should diarrhœe go on to this stage, it is rarcly cured, but above treatment may be perse. vered with for some time.

Ferri sulph. is the best remedy.

Egg-bound.-Sometimes on account of the large size of the egg a hen or other bird is unable to expel it from the oviduct. $\Lambda$ little olive oil should be passed up, taking care not to break the egg; at the same time fomenting the 'vent ' with warm water so as to relax the tissues around. 'This is best accomplished by sitting the hen over a bucket of steaming bran.

Elephantiasis or Scaly Leg.-It is duc to a parasite of the mange order, and is best treated as such. 'I'o facilitate the operation of a sulphur ointment or lotion, the legs should 
be first washed with strong soda water, to remove the dense masses which accumulate to such an extent as to have given the synonym above employed.

Feather Eating.- Is a consequence of indigestion this is at times very prevalent, especially during hot dry weather, and where the drinking water has become contaminated or hot.

It is thought to be due to parasitic invasion of the skin about the feather root. The same remedies as mentioned above for scaly leg are usually successful. Town-kept birds in narrow runs would appear to commence the practice from sheer ennui, but it is quite possible to keep them in perfect health although confined, if green food and grit are supplied in plenty and hard corn added to the kitchen fragments, which consist too often of bread and potato to such an extent as to either set up skin diseases or else diarrhœa.

Gapes.-This very fatal disease is common in young birds of all descriptions, caused by the presence in the trachea and lungs of a worm called the strongylus filaria. These sometimes become so numerous in the windpipe as to entirely fill it, causing the bird to gasp for breath; hence the tcrm 'Gapes.' 'Those affected should be driven into a closed place and made to inhale the fumes from carbolic acid until nearly suffocated; or a feather dipped in glycerin acid. carbol. may be inserted into the windpipe and twisted round; this at the same time anoints the parts and remores some of the parasites when withdrawn.

Where the value of the fowls will warrant it, half a teaspoonful of the glycerine as above may be slowly injected into the trachea by means of a hypodermic syringe. As a preventive the run should be kept as clean as possible, and freely sprinkled with a solution of carbolic acid, or disinfecting porrder and lime freely thrown about the ground.

\section{Giddiness (see Vertigo).}


Indigestion.-Injudicious feeding, such as too much spiced food, meat, or maize, or the want of grit for mastication, often causes indigestion, and may be the forerunner of other diseases.

The symptoms are a want of appetite with lassitude, while the droppings are scanty and unhealthy in character. The food ought to be completely changed, and a comp. rhubarb pill given daily for a week. If the liver seems affected a grain of calomel may be added to the pill, while the water given should be frequently changed.

Leg Weakness is common in young birds of the larger breeds as a result of their rapid growth and want of bony material in the food. The symptoms are constant squatting on the ground and disinclination to move. Bone-dust, lime, $\&$ c., should be given in the food, and 20 -drop doses of syrup

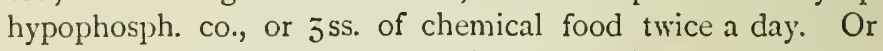
3 to 8 grains of ferri et ammon. citr. once a day.

Liver Disease is evidenced by a sickly yellowish appearance about the head and comb. It is generally the result of over-feeding on too fattening material, such as maize and other starch foods, with highly spiced condiments. It sometimes causes much loss if neglected. Treatment should be directed to reducing the fatty condition and arousing the liver to work by daily doses of calomel gr. j., or a pill as follows :-

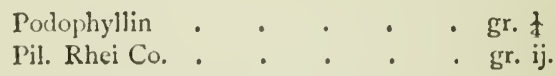

daily for a weck, or until there are signs of improvement. In tuberculous disease of this organ of course there is no cure, and if suspected the best course is to promptly destroy the sufferer and burn the carcase.

Loss of Feathers.-Sometimes the feathers have been pecked out by the other birds. In such cascs the pecked 
parts should be smeared over with sulphur ointment, cut or broken feathers should be removed, and the bird separated from the rest. Generally the loss of plumage is the result of too little green food or of bad housing. Nothing will restore the feathers till the next moult.

\section{Paralysis affecting either of the limbs is incurable.}

Parasites (External). - With ordinary care and attention birds can be easily kept free from parasites. The houses ought to be frequently lime-washed, carbolic acid solution or powder freely sprinkled about, and insect powder on the birds will free them from most pests. Dipping the bird in a weak solution of any soluble disinfectant or parasiticide not stronger than $I-40$ or 50 will be effectual to free them from lice.

Sitting hens should have sulphur flowers under them.

Pip.-Furred tongue, horny tongue, \&c. If very much thickened the tongue should be scraped, and a lotion of liq. sodæ chlor. or borax applied. A dose of pil. rhei co. should also be given daily for a week, from gr: ij. for a young fowl to gr. iv. for a fully grown one. Alum. exsicc. put in the mouth answers the purpose of clearing the tongue.

Rheumatism. - All birds are subject to this affection as a result of bad housing and cold or wet runs. It shows itself by the occurrence of stiff swollen joints; at the same time the toes are cramped and contracted, with painful gait. It is distinguished from leg weakness by the evident pain which it gives. A warm and dry house, and warm stimulating food, to which is added cayenne or other spice, is the best treatment. At the same time the legs should be rubbed with equal parts of turpentine and oil, or 'white oils,' and salicylate of soda given in three to five grain doses.

Roup (Cold, Catarrh, etc.).- Tarious affections of the respiratory organs go by this name. The most prominent 
symptoms are those of an aggravated catarrh, shown by sneezing and gasping, with a discharge from the eyes and nostrils sometimes so bad as to entirely close these. If neglected the disease speedily end's in death. It is very contagious, and therefore the sufferer should be promptly isolated and disinfectants used. The bird must be kept warm and dry, and a dose of castor-oil given; the nose and eyes should be well sponged, and syringed with a solution of liq. sodæ chlor. I-2, and if the nostrils are plugged with the discharge this solution ought to be injected into these, the mouth and throat likewise receiving the same treatment.

Inhalations are found successful in many cases, but unless birds are particularly valuable, for stock purposes, it is almost certain to be cheaper to destroy every affected one. The following is the composition of a favourite, and it is to be put into a TY.II. bottle, and the sufferer's head held over it in such a manner as to compel him to inhale the fumes.

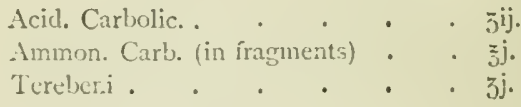

The patient should be held up by the heels for a few minutes night and morning, before the inhalation, as a lot of fluid will cscape from the mouth and nustrils, and afford the medicaments much better access to the diphtheritic membranes, or roupous, to be more exact. As a matter of fact the membrancs may remain in a catarrhal condition for some time and recover, or go on to a croupous or diphtheritic state.

In convalescence the 'Douglass Mixture ' may be given for a time. As recommended elsewhere, if the presence of tubercle is suspected the bird should be destroyed and the carcase burned, in the interests of health and humanity.

Soft Eggs.-This condition is sometimes a result of orer-feeding and want of grit or gravel; hence the remedy is casy-grit, calcined oyster-shells, old mortar, and, in the 
drinking water, some lime, so as to provide material for forming the shell.

Thrush.-Tincture of myrrh and borax diluted with water, to wash the tongue and mouth with twice daily, is the best treatment.

Tuberculous Diseases.-Scrofula, Ulceration, Canker, Consumption, Roup, \&c. Domestic birds are very subject to tubercle in various forms, and nearly all the organs of the body may become affected. The principal symptoms are cheesylooking or calcareous growths, or maybe ulcerations, on any organs, such as lungs, liver, mouth and throat (roup), nostrils. Treatment is of no avail, and the bird should be at once destroyed, and even a healthy-looking fowl when killed ought to be examined for evidence of this disease, and if present it should not be used for human food.

Vertigo.-The birds run in circles or flutter about without control of their movements. Hold the head under a stream of cold water for a time. Give 3 grains of calomel and 10 grains of jalap. Keep on low diet.

\section{TURKEIS}

Sores over the Tail.-Abscess sometimes forms in this situation, causing the bird to depress the tail and suffer a good deal of discomfort. When found to fluctuate under pressure of the finger, the swelling should be lanced at the thinnest point and the matter evacuated by gentle pressure, syringing or bathing with an antiseptic of the lysol class

Diarrhœa is caused by too much green food. Give warm food, chick-peas, and powdered tormentil. Or give 5 grains each of powdered chalk and rhubarb and 3 grains of cayenre pepper. If the flux is not checked, give I grain of opium and I grain of ipecacuanha every four hours. 
Epilepsy.-Give wine, and anoint the head with olive-oil. Much green food must not be given.

Lice result from uncleanness. Dust into the feathers flowers of sulphur or sawdust moistened with benzoline or carbolic acid; and lime-wash the hen-house, adding a little carbolic acid to the wash. A good method is to slake half a bushel of lime, and when cold mix with it Io lbs. of sulphur and I Oz. of carbolic acid. After sweeping out the hen-house, drive out all the birds, close all the doors and windows, and begin at the further end and walk backward, scattering the mixture freely all over the floor and through the air, on to the perches and into the nests, everywhere thoroughly -leaving a dense cloud of medicated dust suspended through the house. Gradually it settles in every crack and crevice, and wherever it reaches a hen-louse or other insect destroys it. If the chickens have roup, leave them in the house; it will not kill nor hurt them, but the sneezing will be terrific, and every particle of mucus that has accumulated in the airpassages and throat will be expelled, and the medicated dust will reach every portion of the membrane and cure it too. In cases of gapes, too, this treatment is likely to be successful. It kills the worms in the air-passages and makes the chicken cough them up, and so cures the case at once.

Pip.-This is commonest among the young ones. To prevent it, add to the drinking water thyme or pepperwort or nigella-seed, and let them often run among green food. The pip-a white horny skin-should be cut from beneath the tongue with a sharp penknife, and taken out. Moisten the part with salt dissolved in wine vinegar, and give nothing to eat. Bread cut in cubes and soaked in vinegar is good later on.

Worms on the Head.-Hang the birds up and search the head thoroughly. If small brown worms are found, which quickly become larger and feed on the head, drench with fisl- 
oil or ol. animalis and ol, picis equal parts, and thercafter rub with this occasionally.

\section{GEESE}

Diarrhœa.-Place the twigs and buds of a young pine tree bruised in the drinking water. Mix bruised thistles with groats as food, and once a week add some tobacco ashes.

Gnats and Flies creep into the little cavities in the ears and nostrils of young geese and kill them. Anoint the ears, in June and July, with linseed or olive oil. If the disease is severe, put barley at the bottom of a deep trough filled with water. The birds in reaching for the food put their heads deep into the water and wash the vermin out. Fresh fern leaves often strewed in the run drive away the insects.

Lice generally affect the young in summer. Rub the affected places with tobacco ashes, oi with a mixture of fish oil and rape oil; anoint the head, and the sides under the wings. If they show themselves on the throats of young geese, which is often fatal, rub the throat with mercurial ointment.

Pip.-Greater pimpernel plant should be steeped in water, the herb given as food, and the infusion as drink. The sore should be cut off, and the wound anointed with unsalted butter.

\section{PIGEONS}

Swelling of the Crop results from eating too much fresh corn. Pay attention to the feeding, and if the crop swells, soak bread in brandy, and give as food.

Pigeons should always be supplied with old mortar or chalk in a box, where they can peck at it, and with a lump of common salt in another vessel. It is a good plan to put some lavender-stalks about the pigeon-house occasionally, and before stocking it, 
The Parasites that infest pigeons are fleas, lice, feather lice, mites, and ticks. Persian insect-powder is efficacious in all cases. Fleas may be killed by sprinkling a little snuff over the birds, and into their nests.

Lice usually attack weak birds. A little powdered sulphur should be dusted among the feathers, and the birds should be kept clean and in good condition.

Mites are very small insects which inhabit the cracks and nooks in the walls of the pigeon-house, issuing at night to feed on the blood of the birds. They sometimes enter the ears of young birds and cause intense annoyance. A drop of oil on the ears, under the wings, or wherever mites are seen, will destroy them. The walls should be smoothed and all cracks stopped. Birch twigs and heath should be given for the nests instead of hay. The house may be whitewashed.

Ticks are larger parasites, infesting generally the head and back. Cleanliness and sulphur are the only remedies.

Feather lice are long, flat, tough insects which cling very tightly between the fibres of the feathers. Their food is the down on the quill end of the feathers.

Canker is a cheesy stinking growth on the mucous membrane of the mouth and throat. It is very fatal to young birds, and is thought to be contagious. Dissect away the cheesy growth with a bit of wood cut like a spatula. Touch the spot with lunar caustic. Give scanty diet and much exercise. If the flesh round the eyes is wounded by fighting, bathe with salt water for several days, and if this does not succeed, try alum and water.

Moulting.-If there are any broken stumps of feathers which the bird cannot remove, considerable suffering results. The stumps must be withdrawn, one by one, with a pair of pincers. Give plenty of good, but not oily, food.

Pouters sometimes orergorge themselves with dry food, which swells in the crop, and is apt to cause death. The crop 
should be opened with a sharp penknife, the mass removed, and the wound sewed up. If skilfully performed, the operation is very harmless.

Roup affects the mucous membranes of the mouth, nostrils, and air-passages. Warmth will cure slight attacks. A copaiba capsule is almost a specific. When the discharge from the nostrils is offensive and purulent, apply to the eye a lotion of nitrate of silver, 5 grains to $\mathbf{I} \mathrm{oz}$. The birds must be kept warm and well nourished; hempseed should be given. Dry roup is known by the dry, husky cough. Give 3 or 4 cloves of garlic every day.

Scouring, or Diarrhcea, is caused in weak birds by want of exercise. Add a pinch of sulphate of iron to the drinking water.

Scrofula show's itself in various forms. As Wing Disease it forms deposits of cheesy and scrofulous matter in and around the joints, especially of the elbow. In early stages tincture of iodine, applied externally, may cure : but in advanced cases the bird should be killed. When it attacks the liver, scrofula causes the formation of white tubercles. The birds lose flesh, and are said to 'go light.' 'They must be destroyed.

Sore Eyes are common among carriers and barbs. A lotion or ointment of silver nitrate should be applied. Among cld birds there is a tendency to form spouts by the turning out of the lower eyelid. These may be removed by cutting them from below upwards with a very sharp pair of scissors.

Vertigo occurs in highly fed birds. Starve for two or three days, and reduce the food afterwards.

Wasting is said to be cured by green food, especially watercress. 


\section{Pigeon Spice Balls.}

Where large numbers of pigeons are kept on the Continent, it is usual to place in the dovecotes little spiced loaves or cones, containing salt, spice, and earth, which the pigeons peck at, and which tend to keep them in health, and induce them to lay more prolifically. These cones are made in this manner: Ten pounds of vetch or other farinaceous seeds are mixed with 2 lbs. of cumin seeds. Separately, sufficient clay is kneaded with water containing in solution 2 lbs. of salt to make a soft dough. This is then thoroughly mixed with the seeds, and the paste is dried in the sun or in a moderately heated oren. Cakes are made from it and kept in a dry place, two or three being left in each doveeote. In the winter especially these form a valuable stimulating food for the birds.

\section{PHEASANTS}

Cold and Roup.-In cold damp seasons, weak broods of young are attacked by catarrh. Remove them to a warm, dry place; give stimulating food, as bread soaked in ale, with cayenne or pepper, and moisten the oatmeal or other soft food with a solution of $\frac{1}{4} \mathrm{Oz}$. ferrous sulphate in I quart of water, using enough to give the meal an inky taste.

Roup is the name given to the disease when the nasal discharge has become purulent and contagious. The diseased birds should be at once removed.

'Gapes' is caused by the presence of parasites or entozoa in the windpipe: young birds are most commonly affected. They keep aloof from the others, have ravenous appetites, and are yet much smaller, weaker, and less feathered than their healthy fellows. At short intervals, the bird stretches its neck and gapes; this apparently unfolds the knot of parasites in the windpipe, and allows of a certain degree of expiration or inspiration. The best method of treatment is to expose the affected bird to the fumes of heated carbolic acid until on the point of suffocation. The bird may le placed in a box with a hot brick, and carbolic acid placed thereon. The birds soon recover from the incipient suffocation, and are almost always freed from the disease. Care must be taken to burn the 
parasites coughed out, and the bodies of any birds which may die of the disease.

Scrofulous Diseases, as tubercles of the lung and liver, can only be remedied by breeding from healthy stock, and removing to fresh, untainted ground.

\section{CANARIES}

The bright plumage is obtained by feeding the birds with food containing a liberal proportion of powdered tasteless Capsicum. Turmeric is also given, and plenty of egg-yolk. Colouring foods consist generally of powdered egg-shells and the mild capsicum. Another good formula is :-

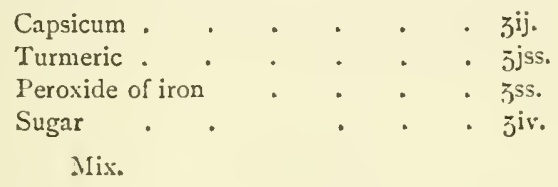




\section{POSOLOGICAL TABLE}

Doses of the most generally used drugs and medicines for animals

FOR cattle the doses are generally about the same as for horses, or rather nore; for sheep the same to the same and a half as for pigs; for cats half the doses given to dogs.

The doses quoted are for average animals of full age. The following table for regulating the doses to younger animals is quoted from Hertwig's 'Arzncimittellehre.'

\section{HORSES}

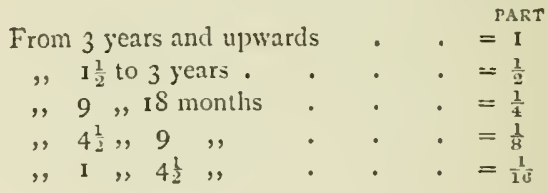

\section{Catti.e}

From 2 years and upwards $\quad . \quad=\mathrm{I}$

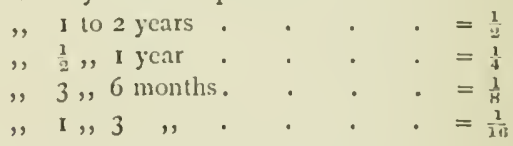

\section{SHEEP}

From 2 years and upwards $\quad . \quad=\mathbf{I}$

$$
\begin{aligned}
& \text { "I to } 2 \text { jears . . . . }=\frac{1}{2} \\
& \text {, } \frac{1}{2}, 1 \text { year . . . . }=\frac{x}{4} \\
& \text {, } 3 \text {, } 6 \text { monthis. . . . }=\frac{1}{8} \\
& \text {, I , } 3 \text {, . . . }=\frac{1}{10}
\end{aligned}
$$




\section{PIGS}

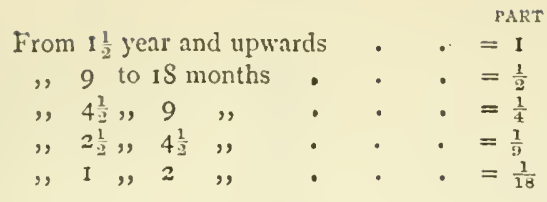

\section{DoGs}

\begin{tabular}{|c|c|c|c|c|c|}
\hline From & $\frac{1}{2}$ to & I year. & • & - & \\
\hline ," 3 & , & 6 months & - & - & \\
\hline$J \frac{1}{4}$ & , & 3 & - & - & • \\
\hline, 20 & , & 45 days. & - & - & - \\
\hline , IO & , & 20, & - & - & • \\
\hline
\end{tabular}

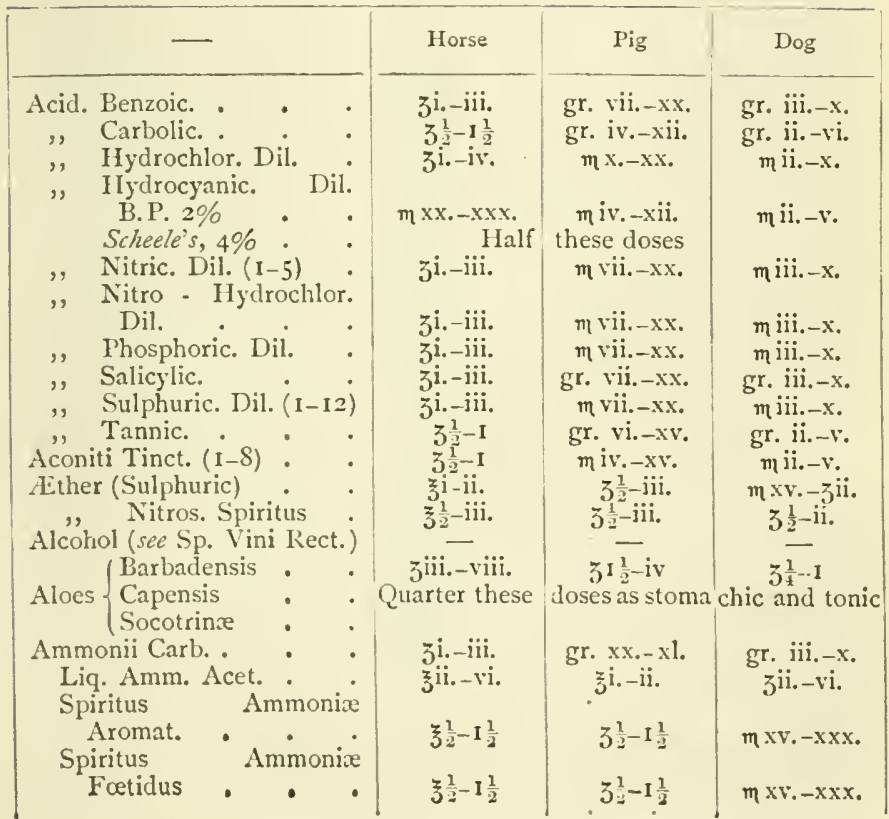




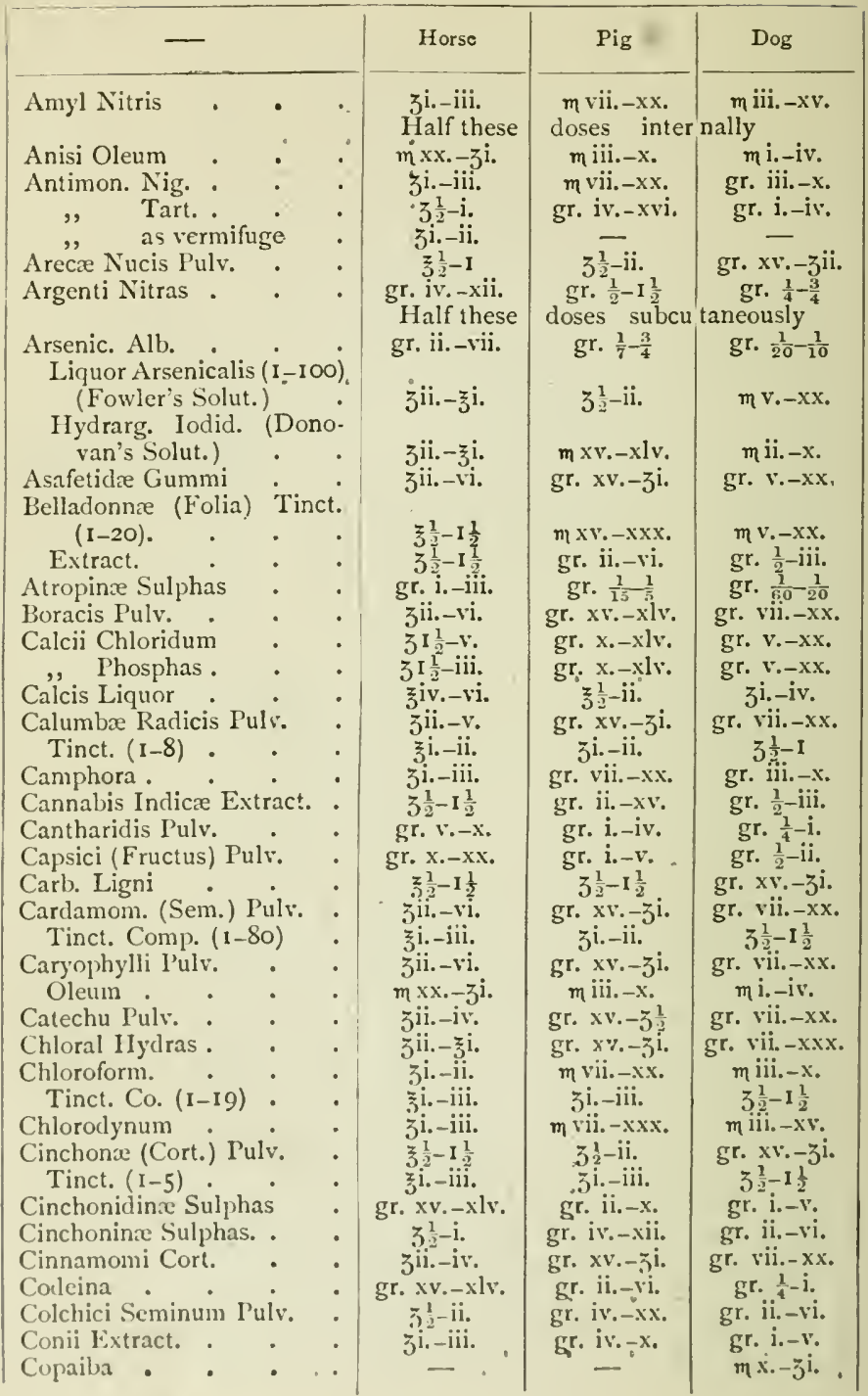




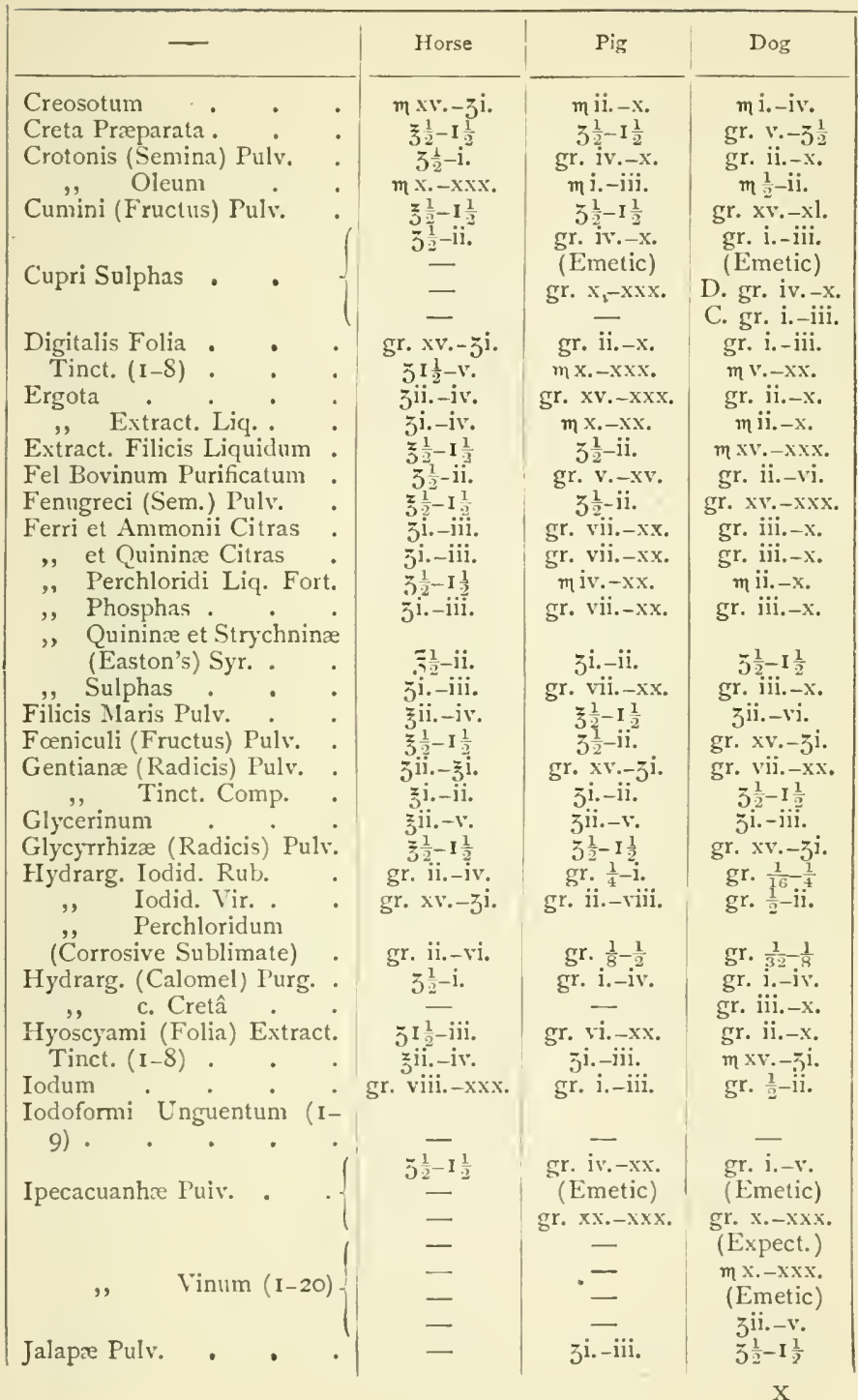




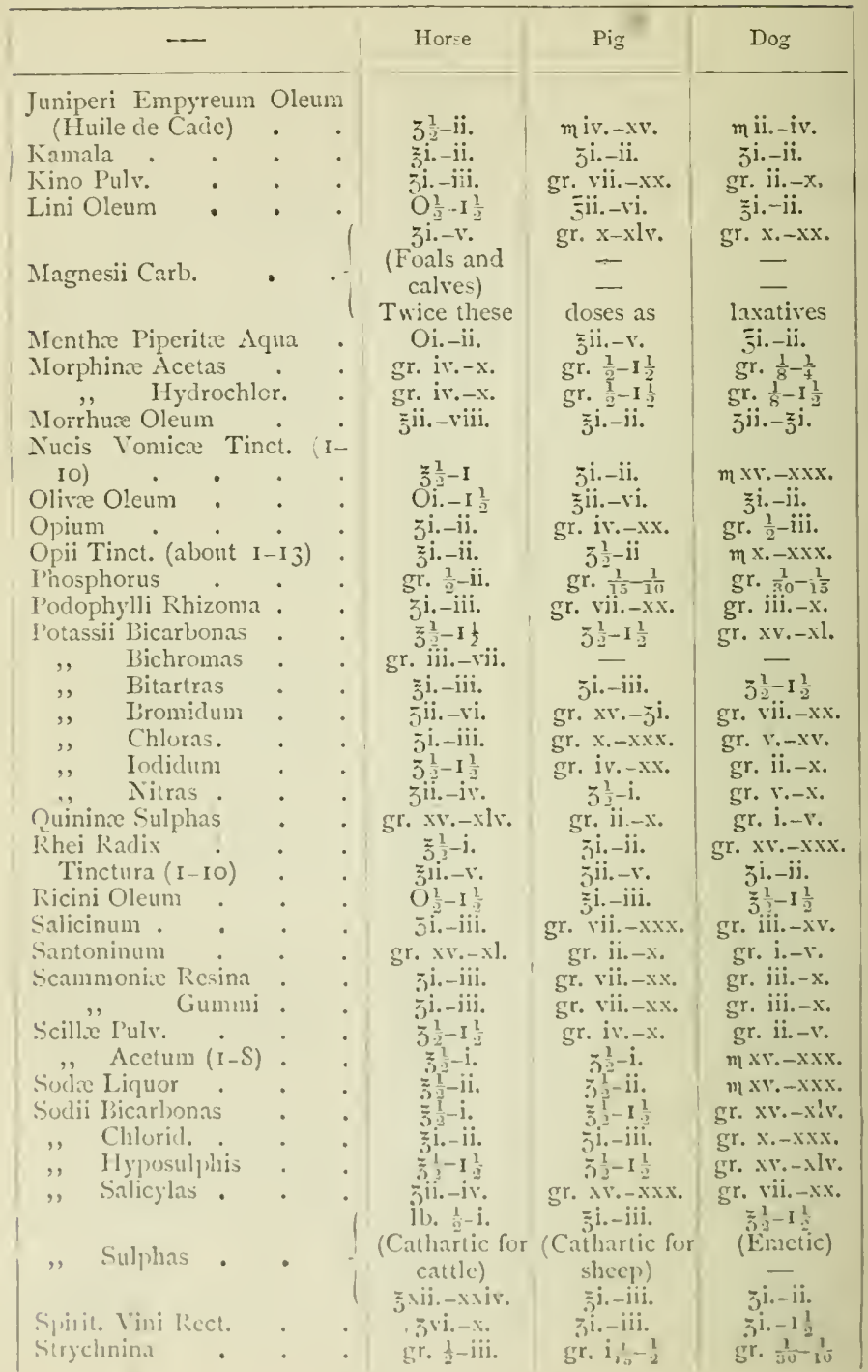




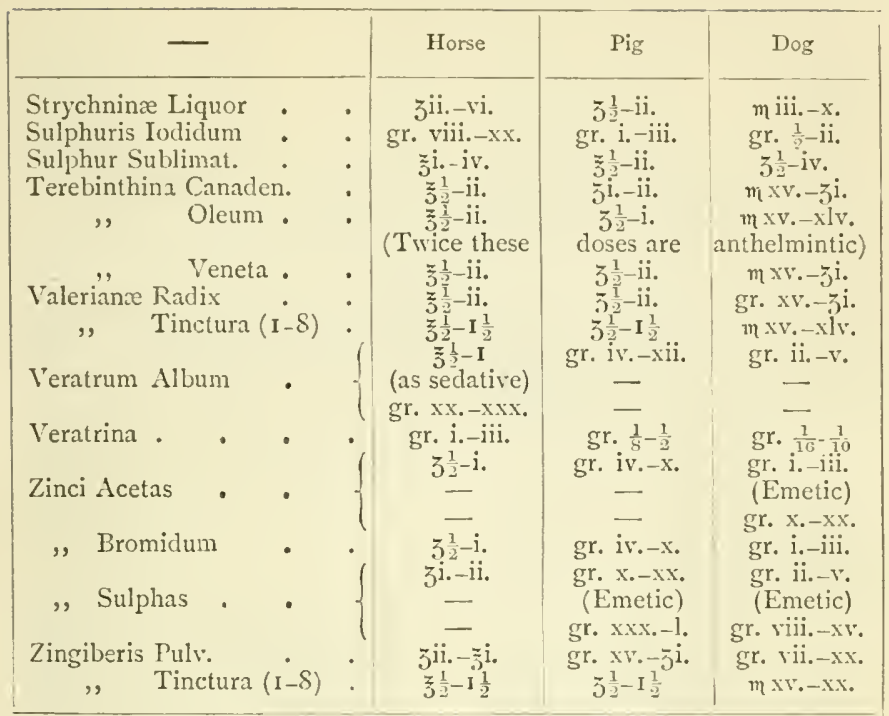




\section{MISCELLANEOUS VETERINARY FORMULAE}

THE following have been contributed at various times to 'The Chemist and Druggist,' and are quoted here for their possible occasional usefulness.

APPETISING POWDER FOR HoRsES.

Cream of Tartar . . . 3 j.

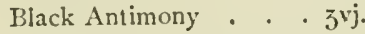

Common Salt . . . ziv.

Powdered Gentian. . . క ziv.

, Juniper berries. żiv.

, Caraway . . 3 j.

, Mustard Seed . „̧j.

Oatmeal . . . . žjj.

Mix.

Dose. - A tablespoonful with each feed thrice daily.

Asthia Cure for Canaries.

Tr. Capsici . . . . 引j.

Spt. Chloroformi • • ‘ jij.

Ferri Ammon. Cit. .

Aq. Fœniculi ad . . . §yj.

M.

A few drops to be put on a lump of sugar in the cage every day.

IBI.ACK-LEG AND BLACK-OQUARTER

DRAUGHT FOR COWS.

Sodii Sulphitis . . . 3j.

Sodii Salicylat. . . . .j.

^ף. . . . . . 3ij.

Solve.
To be given every six hours.(Cressuell.)

Blistering OiNTMexts.

I.

Pulv. Cantharid. - . . $3 x v$.

Pulv. Euphorbii • . . $\int_{3}^{z} v$.

Ol. Olive Comm. . . . 胧x.

OI. Terebinth. . . . $z^{x} \mathrm{ij}$.

Digest for 24 hours, then add

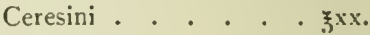

Resin. Flav. . . . ¿ $v^{v}$.

melted by the heat of waterbath, and stirred until creamy.

II.

Pulv. Cantharid. . . ₹zk.

Ol. Morrhux Sec. . . . కnviij.

Gum. Thus . . . . 3 ziij.

Cera Flav. . . . . 3

Vaselin. Vet. . . . $z_{3}^{z x i j .}$

Put the cantharicles and the codliver oil on the hob all day, stirring occasionally; then melt the last three ingreelients together and strain into the cantharides mixture; stir well and add

Ol. Origani . . . ₹ss. 
III.

Pulv. Cantharidis . . . ₹xx.

Ol. Terebinth. . . . $\bar{z} x i j$.

Acid. Acet. Fort. . . . కix.

Lanolini . . . . tbijss.

Vaselini . . . . . tbijss.

Mix the first three, and allow to stand for twenty-four hours; then add the lanoline and vaseline, melted on a water-bath, and mix well, stirring until cold.

\section{(Label.)}

Ilorse Blister, as approved by leading veterinary surgeons. Of all the preparations for blistering horses, this compound is the most highly esteemed, being certain in action, curative, and thoroughly reliable. The blister may be used for sprains, thickened tendons, wind-galls, soft enlargements, and in all cases where the use of a vesicant is indicated by the reterinary attendant.

Directions. - Spread the ointment upon a linen cloth and apply to the affected part, allowing it to remain on all night; then dress with hog's lard.

This label is also suitable for Ung. Hydrarg. Iod. Rub.

\section{Blistering Tinctures.}

I.

Cantharides, powd. . . §jss.

Camphor . . . . . 3 j.

Cochineal . . . . gr. $x$.

Spirit . . . . . q. s.

Macerate in $\tilde{5}$ vij. of spirit for a week, strain, press, and filter, wash- ing the marc with more spirit to make ǰviij.

II.

Cantharides . . . 3iij.

Euphorbium Resin . . ऊjss.

Amyl Acetate . . . $z x$.

Spirit to . . . . $3 x x$.

Macerate for four days, filter, and wash the marc with spirit to I pint.

\section{BRAN Mash.}

Put half a peck of bran into a pail and saturate thoroughly with boiling water. Stir well and cover it, and let s:and till of the temperature of new milk. Various ingredients, such as treac'e, honey, sweet ale, \&c., are occasionally added.

Broxchitis POWders FOR HORSES.

Pulv. Nucis Vom. . . . \jj.

,, Cupri Sulphat. . . $\xi_{j}$.

,, Acidi Arseniosi. . 引j.

M. et div, in pulv. xij.

One powder to be given every night and morning.

BULL-BUR.TT REMEDY,

Pot. Nit. . . . . . 3iv.

Pot. Bicarb. . . . $3 \mathrm{j}$.

Mag. Sulph. . . . కxiij.

M. Ft. haust. Bis terve die ex aqua.

A cooling diet should be given, and an injection into the sheath of

Zinci Sulph. . . . . gr. x.

Aq. Dest. ad . . . . Oj.

Sxpe utendum. 
CALF-MEAL OR MILK Substitute.

Freshly ground Linseed. 7 lbs. Barley Meal . . I I lbs. Wheat Meal . . I4 lbs.

Mix.

A small quantity is made into a thin paste with cold water, then boiling water is poured upon it.

\section{Calving-Drexcil.}

Pulv. Ergotie . . . 3j.

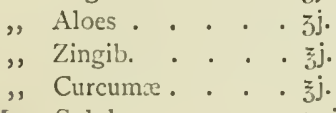

Mag. Sulph. . . . . $\overline{3} x i j$.

M.

To be given in a quart of hot alc or thin gruel within two hours after calving.

Canadiax Oil.

Spirit of Tar . . . 3 j.

Paraffin Oil to . . . $\overline{3}$ viij.

Mix.

This mixture is reputed in Canada to remove dandruff and dirt, leave the skin soft, white, and clean ; to cure the worst case of mange in the world, kecp the hair soft and silky, stop all irritation and rubbing, to be uscful as a preventive against flics, destructive to lice, to take stains out of white loorses, and keep ulack horses from fading out.

CArizunctio POWDer for Cows. lulv. Nuc. Vom. . . . $\mathrm{ij}$. Ammon. Carb. . . . $j^{\mathrm{ij}}$. Ferri Jeroxicl. . . . $\mathbf{j}_{\mathrm{ij}}$ I'ulv. Gentian. . . . §j. M.
To be given in a pint of water twice daily.-(Cressivell.)

\section{Castration Oil.}

Gum. Benzoini . . . 亏うj. Acid. Carbolic. . . . j j.

Rad. Anchuse . . . . $\xi s$.

Ol. Olive . . . . Oj.

Digest for several days in a warm place, and strain.

\section{Cattle Caxcer Ointuent.} Acid. Arsenios. . . . $3 \mathrm{j}$. Pulr. Calaminæ . . $\overline{3}$ j. Adipis . . . . . Ft. ung.

\section{Cattle Food axd Spices.}

I.

Ground locust beans, 50 ; linseed cake, 50 ; coarsely ground liquorice, 5 ; gentian, I ; fenugreek, 3 ; and cumin, I.

II.

Aniseed, allspice, cumin, ginger, liquorice, turmeric, of ench equal parts.

III.

Fenugreek, 500 ; juniper lerries, I00; fennel seed, So ; linseed, I50; licarbonate of soda, 100 ; gentian, I00; ginger, I00; common salt, 50 ; sulphate of soda, 100 ; asafe. ticla, 5 parts. Mix the whole, after powdering, and sift. $\Lambda$ tablespoonful is the dose.

No. I. is a food; Nos. 11. to Ir. are condiments. 
IS.

Ground Linsecd-calie. I cwt.

Locust Meal . . . 56 lbs.

Bran . . . . 40 lbs.

Common Salt . . 2 llos.

Sulphate of Iron. . . I lb.

Black-pepper Giruffs . 2 lbs.

Fenugreck . . . 2 lbs.

Mix thoroughly.

\section{Cilloronye (Veterinary).}

Extract of Indian Hemp . $3^{i j .}$

Capsicin . . . . . gr. r.

Oil of Peppermint . . . 3jss.

Rectified Spirit . . . $3^{i j}$.

Rub up the cxtract and capsicin with a little spirit, and gradually add the rest of the latter; then add the oil and the following :-

Methylated Chloroform . $3 j$ j.

Methylated Ether . . 3j.

Mix.

Separately prepare the following nixture:-

Acetate of Morphia . $3 j$ j.

Dilute Acetic Acid • . 3jss.

Distilled Water. . . . ziv.

Dissolve by heat, and add to

Comp. Tragacanth Powd. $3^{\text {iij. }}$

Treacle . . . . . . इx.

Liquid Ext. of Liquorice. 3 ij.

To this mixture gradually add the chloroformic solution, shaking well after each addition.

The dose of this preparation for a horse is $z_{5}^{\text {ss. to }} \bar{z}$ j. Ten minims of acid. hydrocyanic. dil., B.P., may be addled to each ounce if desired.
Chishire Red Bottle.

I. Boracis . . . . $\frac{3}{3} \mathrm{xij}$.

P. Potass. Nit. . . . zriij.

Tinct. Opii . . . . इvj.

Spt. Camphor. . . . $\overline{3} x$.

Kas. Santal Rub. . . . 3ij.

Ol. Origani . . . .

Tr. Capsici . . . . ziv.

Aq. Fers. . . . Cong. j. dix.

Allow to stand a day or two, and filter.

\section{(Label.)}

The Fanous Cheshire Bottle.

The properties and closes of this highly prized medicine are as fullows :-

For a Coñ Blozine or Hozen:Two wineglassfuls in a pint of mint tea, hot.

Ret Hater or Black. - Two wineglassfuls in a pint of warm milk or whey.

Bloody Urine.-Two wineglass. fuls in a pint of linseed oil.

Diarrhace or Scouring.-Three wineglassfuls in a pint of milk, previously boiled with a little flour.

Fellon Cold or Influenza. - Three wineglassfuls in a pint of warm treacle-water.

Colic or Gripes in Conu er Hurse. - Quarter-pint in a pint of hot water with wineglassful of turpentine, rubbing the loins with same.

Garget or Doiunfall (Gorgle) in Uider.-Apply the Cheshire Bottle warm.

Mill: Fever.-Quarter-pint with one ounce of alum in a quart of barley-water. 
Diarrha:a or Dy'sentery (Gitrr) in Calves. - A large tablespoonful (first dose) with one ounce of castor oil in half-pint new milk; (second dose) in half-pint new milk and halfounce chalk.

Colic Draughts for liorses.

I.

Opii . . . . . 3j.

Spt. Ether. Nit. . . „̋jij.

Chloroformi . . . . $3^{\mathrm{ij}}$.

Aq. ad . . . . . . 3yj.

Rub down the opium with some of the water, dissolve the chloroform in the spirit; add, and make up to $6 \mathrm{oz}$.

For one dose to be mixed with as much water.

\section{For Simple Colic.}

Chlorodyni . . . . zij.

Spt. Ether. Nit. . .

Ol. Lini . . . . Oj.

M.

Give at one dose, and repeat in two hours, if necessary.-(Hoare.)

111. For Flatulent Colic.

Creolin . . . . . ss. $^{\circ}$

Ol. Terebinth. . . . zij.

Spt. Ammon. Arom. . . 3ij.

Tr. Asafetida . . . . ;ij.

Ol. Lini . . . . Ojss.

M.

For one duse. - (Hoare.)

\section{Colchicum Potsonisg.}

Cresswell reconmenclis the fol. lowing dranglut to be administered in a pint of gruel to cows every four hours for three or four successive times :-

Solution of Ammonia . . $3 i \mathrm{j}$. Brandy or Whisky. . . živ. Mix.

CONDITION POWDERS FOR IIORSES.

I.

Nitre . . . . 产iv.

Sulphur . . . . . ziv.

Powdered Gentian . . . 亏j.

, Fenugreck . . $\bar{j}$ j.

" Liquorice . . ऊiv. Mix.

A tablesponful for a doze.

11.

Pulv. Gentianre. . . . ${ }_{3}^{2} \mathrm{ij}$.

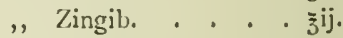

, Fenugræc. . . . 3 ij.

, Glycyrrhiz. . . \jij.

, Pot. Nit. . . . zij.

M. et div. in pulv xij.

One morning and evening.

111.

Sulphur. . . . . . ttj.

Pulv. Glycyrrhiz. . . . „̋xij.

, Pot. Nit. . . . కviij.

, Antim. Nig. . . $5 \mathrm{~s}$.

, Anisi . . . . ₹ij.

, Nucis Vom. . . . zij. II.

A tablespooniul for a dose.

IV.

Prize Medal Condition Poivter.

Pulv. Gentiana . . . siv.

, Pot. Nit. . . . 引j.

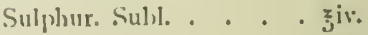

Pulv. Zingib. . . . siv.

, Antim. Nig. . . ऊiv. 
Pulv: Kesin. . . . . . zij.

„ Fenugrac. . . . žij.

, Capsici . . . . 亏

", Serpentarii . . ₹ $\tilde{3}^{\mathrm{ij}}$.

, Sodii Sulphat. . . „ix.

", Lini Placent. . . lbj.

M.

A small tablespoonful twice a day in the feed.

Cough Balls for Horses. l'ulv. Camphorie . $\quad \bar{j}^{5 s}$. , Antim. Nig. . . 35s.

,, Scillæe . . . $j^{\mathrm{ss}}$.

, Digitalis . . . ¿i.

" Zingib. . . . 3j.

,, Pot. Nit. . . . jij.

Mel. . . . . . q. s.

Make a ball.

One ball to be given morning and evening.

COUGH-DRALGIIT FOR HOFSES.

Oil of Anise. . . . . $\eta \mathrm{v}$.

Camphor . . . . gr. xx.

Extract of Liquorice . . 3j.

Tincture of Opium . . $3^{\text {iij. }}$

Spirit. . . . . $\tilde{5}^{\text {SS. }}$

Water to . . . jiij.

Dissolve the oil and camphor in the spirit, and add to the other ingredients, previously mixed well.

COUgh-MIXTURe For DOGs. Tr. Belladonnx . . . 亏̃s. Syr. Scilliz . . . . ₹ss. Tr. Camph. Co. . . . żi. Aq. ad . . . . . žj.j.

M.
Give two teaspoonfuls three times a day.-(Moare.)

\section{Cough-Powders.}

I.

Useful in the simple coughs of horses depending on catarrh :-

Pulv. Camphore . . 3iij.

Potass. Chlorat. . . . zjss.

Pulv. Fol. Belladon. . . ̃jjss.

Pulv. Anisi . . . . 弓̌ij.

Div. in pulv. vj.

Give one twice a day in the food.

II.

For chronic cough in the horse :Pulv. Fol. Aconiti. . . 3 v. Pulv. Digitalis . . . 3iv. Arsenic. Alb. . . . gr. iv.

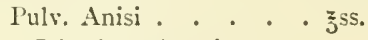

Div. in pulv. v.j.

Give one every night in the food. (Hoare.)

Counter-irritant iN Acute INFLAMMATION,

Spirit . . . . . $3 x$. Spirit of Turpentine . . $z^{x}$. Solution of Ammonia . . 亏ir. Oil of Origanum . . . $\bar{s}$ ss. Mix.

Apply every three hours.

Cud Balls.

Pulv. Rad. Althex . . 亏 j. ,, ,, Glycyrrh. . . „̋j.

, Gum. Acacix . . 亏jj.

,, Sal. Communis. . 3 ij.

Make a stiff mass with water, and cut to suitable sizcs. 
Curb Lotion.

Corrosive Sublimate . . jj.

Potassium Iodide . . $3 \mathrm{j}$.

Proof Spirit . . . . 亏 j.

To be rubbed in at intervals of two to three days till applied eight to ten times.

\section{Devonshire Oils.}

Spirit of Turpentine . $\overline{3} x$.

Camphor . . . . ₹ss.

Dissolve, and add

Solution of Anmonia . . 亏̌j.

Tincture of Opium. . . $\breve{\zeta}^{\text {ss. }}$

Put in a wine-bottle and fill up with the following mixture :-

Soft Soap . . . . . llij.

Boiling Water . . . Cong. j.

Armenian Bole . . . . jjss.

Mix. Labcl, 'Shake the bottle.'

\section{Diapente.}

Gentian . . . . 3iv.

Turmeric. . . . jiv.

Fenugreek . . . . ziv.

Ginger . . . . . 3is.

Anise . . . . . jjss.

Cloves . . . . . jjss.

Caraway. . . . . jjss.

Mix.

DisRrilda or Scour in Cattes. Ponitir.

Mag. Carb. Levis . . . इxrj.

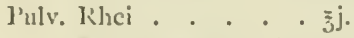

", Pulv. Gilycyrrh. Co. כasts.

" Zingil. Com. . . . $\mathrm{j}$.

J'crri Carb. Sacch. . . „̋ss.

M.

A teaspoonful to be given three times a day. This is especially serviccable when the food sours.
Nixture.

Pulv. Ext. Hrematox. . 3iij.

Cretæ Præparat. . . \弓j.

Chlorodyni . . . . $3^{\mathrm{ij}}$.

Syrup. Zingib. . . . „ „jss.

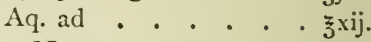

II.

Dose.-Half a wincglassful every four hours.

\section{Distemper Mixture.}

Pot. Chlorat. . . . $3^{\mathrm{ij}}$.

Iiq. Ammon. Acet. . . כ̌j.

Spt. Ether. Nit. . . $3^{\mathrm{ij}}$.

Tr. Hyoscyan.. . . . $3 \mathrm{jj}$.

Aq. ad . . . . . 5iv.

$3 \mathrm{j}$. to $\mathrm{jij}^{\mathrm{ij}}$ ter in cilc.

\section{Distemper Pills.}

Quin. Sulph. . . . gr. j. Pulv. Ipecac. . . . . gr. $\frac{1}{8}$ Ext. Gentian. - q. s. ut ft. pil.

$A$ pill to be given night and morning.

Distemper I'OWDER.

Potass. Nitrat. . . . . $5^{\mathrm{ir}}$

Antim. Nig. . . . Jij.

Sulphur . . . . . jj.

I. Feniculi . . . $5 \mathrm{j}$.

Mix.

Ten to thirty grains for a close, according to the size of the dog.

\section{Dog-Wasir.}

Saponis Mollis . . . . Sacch. Ust. . . . . १. s. Ol. Mirbani. . . . x. Aq. ad . . . . . Oj. II. 


\section{Driffield Oils.}

A preparation for the same purposes as this is macle by mixing together in a $4-1 b$. jar $15 \mathrm{oz}$. of linseed oil and $5 \mathrm{oz}$. of spirit of turpentine. Add, with constant stirring, 1o drachms of strong sulphuric acid, and after a few hours a pint of water. Allow to stand all night, decant the oil, and add an ounce of spirit of tar to it. Take care that the oil does not froth over the jar when adding the vitriol.

Draught For Piles in Cons.

Ext. Taraxaci Liq. . . živ.

Ammon. Chlorid. . . . 弘.

Pulv. Gentian. . . . 引 jj. II.

After a purge of Glauber's salts, give the above twice daily in a pint of warm water. -(Cresswell.)

\section{EMbRocation Powder.}

(For horses' broken knees.)

Plumbi Acet. . . . そ̋j.

Zinci Sulph. . . . 亏 j.

Cretr Rub. . . . . . jj.

Misce bene.

This quantity (two tablespoonfuls) to make a quart of lotion.

\section{Equinocure.}

( $A$ good tonic.)

Sodii Bicarb. z $3 x i j$.

Ferri Peroxid. . . . $5 \mathrm{yj}$.

Pulv, Fenugræe. . . . ̌̌vj.

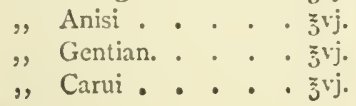

Pulv. Potass. Nit. . . . „xij.

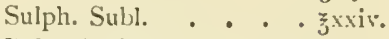
Pulv. Antim. Tart. . . žiij.

Mix and sift.

Dose. - A tablespoonful night and morning with the feed.

Ewes: ANTISEPTIC Syringixg LOTIOX.

Acid. Carbolic. . . . 气j.

Glycerini . . . . . żiij.

Aq. . . . . . . $\mathrm{zx}_{\mathrm{x}}$

II.

Directions. - The whole quantity to be mixed with a quart-jugful of warm water and used to syringe out the womb when the afterbirth is retained.

Ewes' Stimulating Mixture.

(A substitute for spirits.)

Ammon. Carb. . . . jj.

Tr. Zingib. . . . . $\bar{z}^{\mathrm{sS}}$.

Spt. Chloroform. . . $\bar{z}^{\mathrm{ss}}$.

Inf. Gent. Co. Conc. ad . $3^{\text {vj. }}$ II.

Dose.-For a ewe, half a wineglassful in twice as much water; and for a lamb a dessertspoonful.

Ewes' Sootilixg Mixture.

Ext. Ergotre Liquid. . . $z^{5 s}$.

Tr. Opii . . . . . $\xi^{\mathrm{ss}}$.

Spt. Etheris . . . $\tilde{5}^{\mathrm{SS}}$

Tr. Lavand. Co. . . . $\bar{z}^{\text {ss. }}$ M.

This makes two doses. When the afterbirth is retained repeat the dose after two hours. 
EYE-WATER FOR IIORSES.

A saturated solution of boric acid coloured with liquor cocci.

Fellow-Chine or Cow Lumbago DraUght.

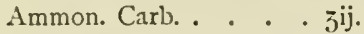

Potass. Bicarb. . . . 引j.

Pulv. Gentian. . . . . ₹̌j.

Pulv. Zingib. . . . \jj.

M.

To be given in a pint of gruel twice a day.

\section{Liniment.}

Lin. Belladonn. . . $z^{\mathrm{ir}}$

Lin. Ammon. Co. . . . 亏̄iv.

M.

(Cressivell.)

Fever Balis for IIorses.

Pulv. Pot. Nit. . . . . žviij.

, Camph. . . . . 3ij.

, Antimorial. . . žjiss.

, Resin. . . . žij.

Theriace. . . . q. s.

Make a mass, binding it with powdered gum, and divide into 5-drach:m balls.

Fever Draugitt for horses.

Spt. Kther. Nit. . . . „j.

Liq. Ammon. Acet. . . $5 \mathrm{~s}$.

II.

To be given before a hot nıash supper.

FI.EMING'S TINCTURE OF Acovitr.

The I3. I'. tincture is generally orlererl in this book, liut as Fle. ming's tincture is often orlered in veterinary practice, we append the formula : Isruised aconite root $1 \mathrm{tj}$. ; macerate with $z_{3} \times v j$. of rectified spirit for four days; strain and treat the aconite by percolation with more spirit until the tincture obtained amounts to $3 x x i v$.

\section{Fly aNd Maggot Oils.}

\section{I.}

Spirit of Turpentine . $5 \mathrm{v}$. Spirit of Tar . . . 5 v.

Corrosive Sublimate . zss. Muriatic Acid . . . zss.

Butter Milk or Water . Cong. ss. Mix.

\section{II.}

The following is a modification of a formula suggested by Professor Robertson :-

Corrosive Sublimate . $3 \mathrm{j}$.

Methylated Spirit . . ₹ sviij.

Spirit of Tar . . $\check{3} x$.

Powdered Quillaia. . žij.

Water to. . . . Cong. j.

Powder the corrosive sublimate, and shake with the n:ethylated spirit until dissolved; then add the quillaia and a pint of water. Macerate overnight, and add the spirit of tar and rest of water; occasionally shake well for a day or two, and strain.

Directions.-To prevent the fly striking, and for maggots: Mix two tablespoonfuls with a winebottleful of cold water.

To kill Lice.-Mix three tablespoonfuls with a wincbottle of cold water and rub on with a brush.

For Mansc. - Mix four tablespoonfuls with a winehottle of cold water, and well rub in the mixture with a brush every day until cured. 
FLY POWDER,

Plumbi Ox. Rub. . . Itss. Plumbi Alb. . . . lbjss.

Pulv. Umber Ang. . . ttij.

Flor. Sulph. . . . . ttij.

Pulv. IHelleb. Alb. . . Itij.

O1. Animalis . . . $3 \mathrm{ij}$.

Ol. Yicis . . . . $x_{3} \mathrm{ij}$.

I. Ft. pulv.

To send out in I lb. packets labelled as stated on p. 209.

\section{FOOT-ROT PASTE.}

Cupri Sulphat. . . . ziv.

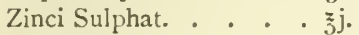
Pulv. Tragacanth. Co. . $3 \mathrm{j}$. Acid. Carbolic. . . 3iij.

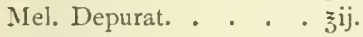
Aq. . . . . . . q. s.

II. Ft. pasta.

FOOT-ROT POWDER.

Camphor. . . . . Cupri Acetat. . . . . 1bj.

Reduce to fine powder and mix intimately.

Directions. - To be applied to the affected parts, and smeared with reterinary vaseline.

Gapes in pueasants.

Sulphate of Iron . . . 5j.

Capsicum . . . . zss.

Fenugreek . . . . 亏j.

Red Sanderswood . . . 亏 j.

Liquorice . . . . $3 \mathrm{ij}$.

Treacle . . a sufficiency.

Mix the powders, and make into a soft pill-mass with the trcacle.
Garget White Oils.

Liq. Calc, Saccharat. . . $5^{\text {iij. }}$ Aq. ad . . . . . 气xx.

Misce et adde

Ol. Lini . . . . $\xi_{3}^{x}$.

Shake well.

To be applied frecly to the udders.

\section{Garget OINTMENT.}

(For inflammation of the udders of cows and ewes.)

Ung. Camphoræ . . . . . j.

Ung. Hydrarg. . . . . 3j.

Ung. Petrolei Vet. . . žrj. II.

Directions.-Gently rub or work the ointment over the udders for five minutes, after which the udders should be wiped carefully with a soft and clean rag.

Grease axi Cracked Heels OnNTheNT.

Sulphur. Subl. . . . . „3j.

Plumbi Acetat. . . . . $\tilde{s}^{5 s}$.

Creolin . . . . 亏5s.

Ol. Eucalypti . . . . $z^{5 s}$.

Vaselini . . . . o jiv.

Lanolini . . . 5iv.

M. Ft. ung.

Apply twice daily. - (Hoare.)

Greex Ointmext.

Resin Ointment . . . $\tilde{3} \mathrm{v}$.

Verdigris. . . . $3^{\mathrm{ij}}$.

Spirit of Turpentine . . 3 ij.

Mutton Suet . . . 1tij.

Oil of Origanum . . . $3^{\text {iij. }}$

Tincture of Iodine . . $3^{\text {iij. }}$ Mix. 
This is a good remedy for scratches, hoof-evil, cuts, \&c., and is also good for fistula, after the rowels are taken out, and a good healing ointment for any purpose.

Gripe DRENCh.

Chloroform . . . . కiv.

Pimento . . . . . žss.

Camphor. . . . . इss.

Opium . . . . . 弓ss.

S.T.R. . . . . . Oj.

Capsicum . . . . そj.

Macerate the opium and capsicum in the spirit for seven days, filter, and mix the other ingredients. Dose, $\mathbf{I} \frac{1}{2} \mathrm{oz}$., with $\mathbf{I} \mathrm{oz}$. of spirit of nitre, in a pint of warm gruel.

\section{Healisg Lotion.}

(For sprains, bruises, sore throats, cuts and wounds in horses.)

Liq. Plumbi Subacet. . . zjj.

Ol. Eucalypti . . . కiv.

Aceti . . . . . . . 核 $v$ ij.

Vitell. Ovi . . . . ij.

Ol. Sesamx ad . • • „ „xx.

Rub the egg-yolks in a mortar with the sesame oil, add the euca. lyptus oil, then the lead and vinegar mixed together, and make an emulsion.

\section{IIEAVES REMEDY.}

Cinarla Balsam . . . žir.

Copraila . . . . . . $\overline{3}^{\mathrm{j}}$

Calcined Magnesia, a sufficiency.

Nake a mass, and divide into half-ounce balls.

Dose.-One ball night and morning for cight days.
HoRSE BALLS.

Barbadoes Aloes . . . ̌nviij.

Ginger . . . . . 弦.

Soap . . . . . 3j.

Treacle . . . . . q. s.

Melt at a moderate heat to form a mass. Dose from $3^{\mathrm{v}}$. to $3 \mathrm{x}$.

J. Bell \& Co.'s formula (Pharm. Joum. vol. r).

\section{Horse Tice, or E.itice,} is generally composed of an equal mixture of ol. anisi and ol. rhodii. To catch colts, this mixturc is rubbed into the hands or upon the coat. sleeve.

Restive horses are sometimes rendered quiet by smearing the bit with it. It does not answer equally well with all individuals, as some persons have an influence over horses which they cannot themselves ascribe to drugs.

Hoven Draught for Cattle. Creolin. . . . . . jj. Ol. Terebinth. . . . ऊiv. Spt. Ammon. Arom. . . živ. Ol. Lini . . . . Ojss. M.

For one dose. - (Hoare.

\section{IIUSK MiXTURE.}

Ferri l'eroxid. . . . $\mathrm{j}^{\mathrm{ij}}$. Spirit. I'icis . . . . $3 \mathrm{j}$.

Ol. Terebinth, . . .

Ol. Lini ad . . . 3̌riij. II.

A small tablespoonful night and morning. 
Influenza (Pink Eye) Powners.

Pulv. Ammon. Chlorid. . 3iij.

Pulv. Potass. Nit. . . ziij.

Pulv. Glycyrrhiz. • . ̌̌ziss.

Puiv. Sodii Sulphat. . . ̌ $3 x$. II.

Dose.-A teaspoonful in gruel thrice daily.

\section{INFLUENZA BALLS.}

Pulv. Camphor. . . . $3 \mathrm{j}$.

Pulv. Pot. Nit. . . . . $3^{\mathrm{r}}$

Pulv Aloes . . . . 亏jj.

Lini Farinæe. . . . $5^{\text {rj. }}$

Theriacæ. . . . q. 5 .

Ft. mass. et div. in bolos ij.

'Give one ball immediately, and the other three hours after.'

\section{INFLUENZA DRAUGHT FOR HIORSES.}

Chlorodyni . . . . jj.

Spt. Ether. Nit. . . żij.

Liq. Ammon. Acct. . . $\overline{5}^{\mathrm{ij}}$. Aq. ad . . . . $\bar{z}^{x v}$.

M.

This dose is to be given every three hours during the first stage, when much shivering is evident.(Hoare.)

\section{LAME DRENCII.}

Magnesium Sulphate . . 亏iv.

Sodium Sulphate . . కiv.

Diapente. . . . . 亏̌ij.

Nitre . . . . . 3 j.

Sulphur . . . . . 亏̌j.

Feroxide of Iron . . `sss.

Mix intimately.

Dose.- I oz. in gruel.

For Diapente, see p. 314.
LAMBIxg OILS.

Acicl. Carbolic. . . . そss.

Ol. Viridis . . . そ jij.

Ol. Gossypii • . . そ̌xxij.

Directions. - To dress the shape, and to apply inside the passage by means of the fingers in order to assist the delivery of the lamb. If the afterbirth is retained, some of the oil should be introduced into the womb by means of a syringe.

\section{LAMENESS LINIMENTS.}

\section{I.}

Liq. Ammon. . . . . 亏ैv.

Spt. Cansphor. . . . . ̌̌v.

Tr. Canth. ( 1 in 10) . . zjoss.

Sapon. Mollis . . . ziij.

Aq. ad . . . . . . इ $\mathrm{xxx}$.

Dissolve the soap in the water, and add the other liquids.

\section{Restitution Fluid.}

Sodii Chlorid. . . . . zr.

Tr. Capsici . . . . $\overline{3} \mathrm{r}$.

Spt. Camphor. . . . $\overline{3} x$.

Liq. Ammon. . . . . 亏ूx.

Spt. Etheris . . . $3 x$.

Spt. Tenuior. . . . ॅ̌x.

Rub the salt to fine powder and mix with the liquids.

\section{Leeming's EsSence.}

Cantharides. . . . 气viij.

Camphor. . . . . zij.

Euphorbium . . . 污iij.

Oil of Origanum . . . 3 j.

Castile Soap . . . žiij.

Spirit . . . . . Orj.

Digest for 14 days and filter. 
LOTION FOR BROKEY KXEES.

Cupri Sulph. . • • žj.

Gum. Benzoin . . . zij.

Aquæ. . ". . . $3 \times 1$.

Boil in covered ressel and filter.

Lotion for Fouls ix Cattle.

Liq. Hydrarg. Pernit. . そj.

Glycerin. • . . . 弓sss.

Aq. ad . . . . . 3ij.

M.

To be applied to the sores with a soft rag once a day.

\section{MaLt Mash}

is made in the same way as Bran Mash, substituting malt for bran.

Ma.̃ge SMeAR.

Sulphuris Nigri. . . $3^{i j}$.

Olei Cadini . . . . jij.

Spiritus Picis ad . . . そj.

MILK-fEVER POWder FOR COWS.

Pulv. Camphor. . . . jj.

Pulv. Potass. Nit. . . $3^{i j .}$

Pulv. Sodii Sulphat. Exsic. そ̌jss.

II.

This powder to be given thrice daily in water.

\section{MITIRIDATE.}

In Yorkshire this preparation frequently forms an ingredient of cattle-drinks. The formula used Incally is :-
3. Bacc. Lauri . . . \3. j.
I'. Pip. Iong. . . . . 3 j.

P. Sem. Carui . . . . „̧ss.

P. Sem. Anisi . . . 弓ss.

P. Rad. Gentian. . . . そij.

P. Rad. Curcum. . . . కzij.

P. Rad. Valerian. . . . zj.

P. Rad. Zingib. . . . 3 j.

P. Gum. Acacize . . . ̌̌jij.

Bole . . . q. to colour.

Oatmeal Gruel.

Put I lb. of medium oatmeal into a basin and add about $I$ gallon of cold water. Mix thoroughly, let the coarser particles settle, pour off the liquid and bring it to the boil, stirring all the time.

OINTMENT FOR GREASE IN HORSES.

Citrine ointment . . Lard . . . . . zi . Spirit of Turpentine . . $5 s$. Sat. Sol. Nitrate of Copper $3 \mathrm{ij}$. Mix.

OINTMENT FOR HORSES' KNEES.

Mcrcurial Ointment . . sij. Honey . . . . . 5j. Camphor. . . . . $3^{\mathrm{ij}}$. Burned Cork, powdered . jij. Mix.

Oritinext for Sores, Chafes, S.C.

Pulv. Boracis . . . 3j.

Pulv. Carbo. Animalis $\cdot 3^{\text {ss. }}$

Ol. Picis . . . . $m x$.

Ol. Camphor. . . 3j.

Adipis ad . . . . $\bar{j}$.

M. ft. ung. Nocte maneque utend. 
OINTMENT FOR WTARTS OR

ANGLEBERRIES.

Arsenici Sulphid. Flar. . jj.

(ilycerini . . . . $3^{\mathrm{ij} .}$

Lanolini . . . . . $3^{\mathrm{r}}$

II.

Apply to the part night and morning.

PIG POWDERS.

I.

Flower of Sulphur . . . It xxx.

Red Ochre . . . . 1bjss.

Tartarated Antimony . . $\overline{3} x i j$.

Mix. Weigh into powders containing $\frac{1}{2} \mathrm{oz}$. each.

One powder to be given every few weeks.

II.

Pulv: Pot. Nit. . . . ₹viij.

Sulph. Sublim. . . . zriij.

Ferri Oxid. Rub. . . ㅊiv.

P. Curcumæe . . . . इnviij.

I'. Carui . . . . . 三ij.

P. Fenugræc. . . . . $\bar{z}^{x}$ II.

Doses.-From a dessertspoonful to a tablespoonful.

III.

Golden sulphuret of antimony and red oxide of iron, of each, I 5 grains ; common salt, 30 grains; nitre, 40 grains; powdered liquorice, of each, 30 grains. Mix well, and give with the food. The powder may be made in bulk, and given in doses of a teaspoonful.

Purgative Oil for Cattle.

Ol. Crotonis . . . jss. $^{\mathrm{s}}$

Tr. Opii . . . . 3jss.
Ext. Glycyrrh. Liq. . $3^{\text {iij. }}$

Ol. Lini ad . . . $\bar{z}^{\mathrm{xx}}$. II.

Dose.-For a calf a wineglassful, fur a cow two or more.

Purgative Powder for Cows.

Tartar Emetic . . . . jj.

Dried Glauber's Salts . . žriij.

l'owdered Aloes . . . 弓jj.

Mix.

Give a tablespoonful every three hours until the bowels are mored.

PURgative POWDER for HORSES.

Powdered Croton Seeds . Эj.

Crushed Linseed . . . 3j.

Powdered Caraway . . jj.

Mix.

This is a dose for a carriage horse.

Purgatile Powder for Cattle AND HORSES.

Epsom Salts . . . Hbj.

Powdered Fenugreek . . 亏jj.

Peroxide of Iron . . . zsss.

Mix well.

Ren Coxdition Balls.

Ferri Carb. . . . . 3jss.

P. Pot. Nit.. . . . כj.

I'. Ouassia . . . . $3 \mathrm{j}$.

Antim. Sulphuret. . . $3^{\text {ss. }}$

Pulv. Zingib. . . . $\overline{5}^{\mathrm{ss}}$.

Ol. Fceniculi . . . gtt. x.

I.

Mass with linseed meal and simple syrup to form 6 drachm balls.

Dose.-One twice or three times a week. 
Roup Pills for POUltry. I.

Hydrarg. Subchlor. • . gr. j. Pulv. Antimonialis . . gr. j. Pulv. Glycyrrhiz. . . . gr. j. Copaibre . . . . . q. s. Ft. pil.

A pill to be given night and morning.

II.

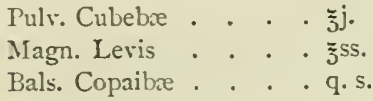

Mass and divide into 3 -gr. pills, a few drops of oil of aniseed being added to the mass to give a distinctive smell.

\section{Sheep Mixture.}

(For cough, fever, \&c.)

Acet. Ipecac. . . . . $\overline{3}^{\mathrm{ij}}$.

Liq. Cocci . . . • $3^{\text {vj. }}$

Chlorodyni . . . . $3^{\text {iij. }}$

Spt. Terebinth. . . . iv: $^{\text {iv. }}$

Liq. Ammon. Acct. ad . $\check{3}$ viij. M.

Eircctions.-A small tablespoonful three limes a day.

Stimulating White Linimext.

Ol. Terebinth. . . . క̌xij.

Camphore . . . . $5 \mathrm{j}$.

Saponis Mollis . . • .

Aq. Destil. . . . . ₹ ₹ij. vil q. s.

Mix the soap with the water; discolve the camplaor in the turpen. tine; rix the two, and loring lown to the desirel consistency with "sater. - (Hoarc.)
Sinine Cramp Powders.

Potass. Brom. . . . 3jss.

Sodii Chlorid. . . . $3^{\mathrm{ij}}$.

Pulv. Glycyrrhiz. • . . ₹j.

M. et div. in puls. xij.

One powder to be giren three times a day.

SWINe Diarrhaga Mixtere.

Tr. Opii . . . . . $\mathrm{j}^{\mathrm{ij} .}$

Tr. Valer. Am. . . . $5^{\text {iij. }}$

Inf. Catechu . . · zij.

Liq. Calc. Sacch. . . . 3iv.

Dec. Quercûs ad . . 亏̄ँvj. M.

Dose. - zj. twice or three times a day.

Throat Lisimext.

(For sore throats in horses.)

Ol. Terebinth. . . . $\mathrm{s}^{\mathrm{j}}$.

Liq. Ammon. Fort. . . $\mathrm{j}^{\mathrm{j}}$.

Ol. Olive . . . . . žj.

M.

(Hoare)

Tinctura Opi Anqosa.

(Suitable for veterinary prepara. tions.)

Boil $\mathrm{I} \frac{1}{2} \mathrm{oz}$. of opium with $5 \mathrm{oz}$. of water for half an hour, replacing any water lost by evaporation ; then make up to $15 \mathrm{oz}$. with water, add 5 oz. of rectified spirit, and after two or three days filter.

TONIC PILls fOR PigeNon.

Ferri Sulplı, Gran. . . jj.

Iulv. Capsici . . . . ilj.

Ext. Nucis Von. . . . gr. vj.

l'ulv. Gentiance . . . js.

Gilyc. Tragacanth.. . . (.s. 
Mix, and divide into 60 pills. Coat with sugar. Allow each pigeon six of these pills per day.

Warbles in Horses aNd CatTle. Zinci Sulphat. . . . . jiij. Liq. Amınon. Fort. . . $3^{\mathrm{rj}}$. Spiritûs Vini . . . ̌̌jss. Liq. Plumbi Subacet. . . うjss. Tr. Aloes et Myrrhe . . Jjss. Aq. ad . . . . . 亏̌viij. M. Ft. lotio.

'To be used twice daily.'

\section{Warble Prevestive.}

Ol. Lini . . . . ”zijss. Ol. Terebinth. . . . jiijss. Tinct. Asafetidre . . . 3 j.

Smear along the back and sides, early in summer, and repeat occasionally during the hot season.

\section{TORM OIL.}

(For worms in lambs' throats.) Spirit of Turpentine . . siv. Linseed Oil . . . . Jiv. Powd. Red Sanderswood. 亏s s. Mix.

A teaspoonful of this to be slowly poured over the animal's throat.

WorM REMEdIES FOR HoRses. I.

Dippel's Oil (Ol. Animal.) $3^{\text {ijjss. }}$ Paraffin Oil . . . . jijss. Powdered Valerian . . 3̌j. Powdered Santonica . . ₹ zij. Mix.

To be given in a corn feed.
II.

PuIv. Acid. Arsenios. . gr. xv. Sem. Lini Contus. . . ₹ss. M.

A powder to be given at sippertime for four or five day's.

III.

Cupri Sulph.

Arsenic. Alb. . . . . zss.

Pulv. Sem. Santonic. . . žj.

Pulv. Glycyrrh. . . . ziv. M.

A tablespoonful in a mash twice a week.

$$
\text { IV. }
$$

Santonin . . . . 3j.

Tartar Emetic . . . $3^{\text {ss. }}$

Physic Mass. . . . ¿ss.

Mix and make a tall.

\section{V.}

Pulv. Antim. Tart. . . $5^{\text {iv. }}$ , Jalapæ . . . 亏̈jss.

", Zingiberis . . $3 \mathrm{ij}$.

, Aloes Barbad. . . zij.

,, Sapon. Ilispan. . $z^{\text {ss. }}$

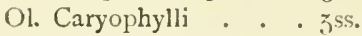

Syrupi Simplicis . . .

Mucil. Tragacanth. . . 亏5s.

Make a mass, and divide into I-oz. balls.

Directions.-Give a ball after a vcry light meal at night, repeating in two or three days if necessary. A tonic ball should be given once a week, or else a tablespoonful of tonic and condition powder every other day to horses which are sub. ject to worms. 
VI.

Sulphur. Nigri . . . 亏jj.

Pulv. Antin. Tart. • . 3j.

Bol. Armen. . . . . 3j.

Mix thoroughly in a mortar.

Directions. - To be given after a light mash.

IVound-nestixg Powder.

Iodoform . . . . . 引j.

Pulv. Acid. Boric. . . .

M.

To be dustcd upon the wounds and sores of horses.

WOUND BALSAM.

Elemi. • . . . 5jij.

Tenice Turpertine. . . Jiv.

Tallow . . . . . jiv.

Lard . . . . . 亏iv.

Cotton Seed Oil . . . 引j.

Carbolic Acrd . . . gr. x.

Alkanet . . . . . q. s.

WOUND STONE.

Iron Sulphate . . . Itij.

Alum . . . . . ttij.

Zinc Sulphate . . . . „్

Copper Sulphate . . 引iv.

Armenian bole enough to

colour it.

Powder the ingredients. Mix and sift.

Put up in 2-oz. packets. To be dusted on the wounds alternate days until the sore appears healthy.

NEWMARKET PIIYSIC-I'ASTE.

This paste was supplicd in the fifties to the leading trainers and turfites-viz. the late John Scott, 'Ihe Wizarel of the North' in racing circles, the late Culoncl l'eel, Count
Batthyani, the late Marquis of Exeter's trainer, the late John Day (Lord Palmerston's trainer), Jem Robinson (the celebrated jockey), Frank Battlis, 'Nat' (Colonel Peel's jockey), Sam Rogers, Job Marron (the rider of Teddington), and many others. The recipe is a good one.

\begin{tabular}{|c|c|c|}
\hline Aloes Ba & nsis & 1bxiijss. \\
\hline Saponis & munis & . Hbivss. \\
\hline Potassa & natis & . $\tilde{5}$ xviij. \\
\hline Ol. Anisi & - & . ऊ $\mathrm{vj}$. \\
\hline Aqure & . & $\cdot \overline{3}$ \\
\hline
\end{tabular}

Cut the soap into small shreds and put into a pan with the water. Ileat, and when thoroughly melted and quite smooth add the carbonate of potash and the aloes. Let it sinmer for some time, stirring fre. quently until the aloes is dissolved. If allowed to boil, the mass will come over before melted. Lastly add the oil of anise, and stir it well in.

A small piece of the mass taken out before the anise is added, and cooled on a slab, will tell whether the paste has licen brought to a proper consistence or not. Twelve drachms of the mass contains $S$ drachms of aloes. The paste used to be supplied to trainers in 3-lb., 4-lb., and $7-1 \mathrm{~b}$. tins.

STOPPIX⿻; roR HORses' IIEELS.

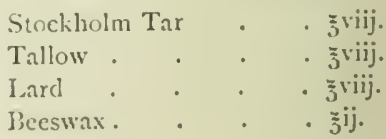

Mclt the last three first, then add the tar, and stir up wcll. 


\section{THE VETERINARY SURGEONS ACT, 44 \& 45 VICT. с. 62 \\ Passed August 27, I881}

WHEREAS it is expedient that provision be made to enable perscns requiring the aid of a veterinary surgeon for the cure or prevention of diseases in or injuries to horses and other animals, to distinguish between qualified and unqualified practitioners :

Be it therefore enacted by the Queen's most Excellent Majesty by and with the advice and consent of the Lords Spiritual and Temporal and Commons in this present Parliament assembled and by the authority of the same as follows :

I. This Act may be cited as the Veterinary Surgeons Act IS\& I.

2. In this Act-

'The Royal College of Veterinary Surgeons' means the Royal College of Veterinary Surgeons incorporated and regulated by a charter and two supplemental charters granted by Her Majesty in the years one thousand eight hundred and forty-four, one thousand eight hundred and serenty-six, and one thousand eight hundred and seventy-nine respectively.

'The Registrar' means the Registrar for the time being of the said Royal College.

'Veterinary surgery' means the art and science of veterinary surgery and medicine.

3.-(I.) The register of members of the Royal College of Veterinary Surgeons directed by Her Majesty's said Royal Charter of $1 S 76$ to be made and maintained, shall be styled the Register of Veterinary Surgeons, and shall be kept as accurately as possible by the Registrar.

(2.) The Council of that College shall cause correct copies of the said register to be from time to time and at least once a year printed under their direction and published and sold, and such copies shall be admissible in evidence.

4. The Royal College of Veterinary Surgeons shall be bound to make provision in the manner permitted by their charters for the examination in England of the students attending the Royal Veterinary College, and in 
Scotland of the students attending the several Scotch Veterinary Colleges, and in Ireland whenever a Veterinary College shall be established in that country, and to admit and register such students as have passed the examination as members of the said Royal College under the provisions of such charters and this Act.

5.-(I.) The Registrar shall from time to time insert in the Register of Veterinary Surgeons any alteration which may come to his knowledge in the name or address of any person registered.

(2.) The Registrar shall remove from the said register the name of every deceased person.

(3.) The Registrar may remove from the said register the name of a person who has ceased to practise, but not (save as hereinafter provided) without the consent of that person.

(4.) Where the Registrar has reason to think that any person registered has ceased to practise, the Registrar may send by post to such person a notice inquiring whether or not he has ceased to practise or has changed his residence; and if the Registrar does not within three months after sending the notice receive any answer thereto from such person, the Kegistrar may within fourteen days after the expiration of the three months send him by post in a registered letter another notice referring to the first notice and stating that no answer thereto has been received, and if the Registrar does not within one month after sending the second notice receive any answer thereto, sucl person shall for the purpose of the present scction be deemed to have ceased to practise and his name may be removed accordingly.

(5.) In the exccution of his duties the Registrar shall act on such cridence as in each case appears sufficient.

6. The power conferred by the said Supplemental Charter of 1876 on the Council of the said Royal College, at a mecting of the Council, at which not less than two thirds of the members are present, and with the consent of three fourths of the members so present, but not otherwise, to remove a name from the Register of Veterinary Surgeons, may be excrcised in respect of any person who is at the passing of this Act on that register, or who is after the passing of this Act placed thereon under the said charter, or this $A c t$, but in the following cases only (that is to say), at the request or with the consent of the person whose name is to be remored, or where a name has lieen incorrectly entered, or has been fraudulently entered or procured to he entered, or where a person registered has, either before or after the passing of this $\mathrm{Act}$, and either befere or after his registration, been convicted, éther in IIer Majesty's dominions or elsewhere, of an offence which, if committel in England, would be a misdemeanour or higher offence, or where a person registered is shown to have been guilty, either before or after the passing of this $\mathrm{Act}$, and either liefore or after his regis. 
tration, and either in IIer Majesty's dominions or elsewhere, of any conduct disgraceful to him in a professional respect.

7.-(1.) Where the Council of the said Royal College have removed the name of any person from the Register of Veterinary Surgeons, the name of that person shall not be again entered in the register except by a resoli:tion of the Council passed under this section, or by order of a court of competent jurisdiction.

(2.) The Council may by resolution passed by a like proportion of their number as is for the time being required for the removal of a name from the said register, dircct the Registrar to restore to the register any name removed therefrom, either without fee or on payment of such fee not exceeding the registration fee as the Council from time to time fix, and the Registrar shall restore the same accordingly.

(3.) The name of any person removed from the said register at the request of such person or with his consent shall, unless it might if not so removed have heen removed by order of the Council, be restored to the register, on his application and on payment of such fee not exceeding the registration fee as the Council from time to time fix.

8.-(I.) The Council of the said Royal College shall, for the purpose of exercising in any case the power of removing a name from or of restoring a name to the Register of Veterinary Surgeons, ascertain the facts of the case by a committee of the Council, the powers of the comnittee being exerciseable by not fewer than three members of the committee ; and the report of the committee, after hearing the person concerned, if he so desires, shall be for the purpose aforesaid conclusive as to the facts, but so that the Council shall form their own judgment on the case independently of any opinion of the committee.

(2.) If in any case the Council determine to remove the name of any person from the Register of Veterinary Surgeons, or not to restore thereto the name of any person, the Council shall, if required by him, state in writing the reason for that determination, and he may appeal to the Privy Council ; and the Priry Council, after communication with the Council of the said Royal College and the appellant, may either dismiss the appeal, or order that Council not to remove the name of the appellant, or to restore his name, as the case may require.

9. A copy of the Register of Veterinary Surgeons for the time being purporting to be printed and published in pursuance of this Act shall be evidence in all cases (until the contrary be made to appear) that the persons therein named are on the Register of Veterinary Surgeons; and the absence of the name of any person from such copy shall be eridence (until the contrary be made to appear) that such person is not on that register: Provided that in the case of any person whose name does not appear in such copy a certified copy under the hand of the Registrar of the entry of the name of 
such person in the said register shall be evidence that such person is on the said register.

10. Every registrar of deaths in the United Kingdom, on receiving notice of the death of any person on the Register of Veterinary Surgeons, shall forthwith transmit by post to the Registrar a certificate under his hand of such death, with the particulars of time and place of death; and on the receipt of such certificate the Registrar shall erase the name of such person from the Register of Veterinary Surgeons, and shall transmit to the said registrar of deaths the cost of such certificate and transmission.

I1. Any person who wilfully prccures or attempts to procure himself to be placed on the Register of Veterinary Surgeons by making or producing or causing to be made or produced any false or fraudulent declaration, certificate, or representation, either in writing or otherwise, and any person aiding and assisting him therein, shall be deemed guilty in England or in Ireland of a misdemeanour and in Scotland of a crime or offence punishable by fine or imprisonment, and shall on conviction thereof be liable to a fine not exceeding fifty pounds or to be imprisoned with or without hard labour for any term not exceeding twelve months.

12. If the Registrar wilfully makes or causes to be made any falsification in any matter relating to the Register of Veterinary Surgeons, he shall be decmed guilty of a misdemeanour, and shall be liable to a fine not exceeding fifty pounds, or to be imprisoned with or without hard labour for any term not exceeding twelve months.

I3. - (I.) Where a person shows that he holds some recognised veterinary diploma granted to him in a British possession, and either that the grant of such diploma occursed when he was not domiciled in the United Jingdon, or in the course of a period of not less than five ycars during which he resided ont of the United Kingdom, or, if he was practising veterinary surgery in the United Kingdom at the passing of this Act, that he has practised veterinary surgery for not less than ten years, either in the United Kingclom or elsewhere, he shall upon payment of the registration fee be entitled without cxamination in the United Kingdom to be registered as a colonial practitioner in the Register of Veterinary Surgeons and to become to all intents a member of the said Royal College.

(2.) Where a person shows that he oltained some recognised veterinary diploma granted in a foreign country, and either that he is not a British sulject, or that if a liritish suliject he has practised vetcrinary surgery for more than ten years clscwhere than in the United Kinglom, or if he was practising reterinary surgery in the United Kingtom at the passing of this $\lambda e t$ for not less than ten years, either in the United Kingdom or elsewhere, and either continues to hold that diploma or has not been deprived thereof ly any cause which disfunalifies him for leing registered uncler this $\mathrm{Act}$, he shall, on panyment of the registration fec, be entitled without examination 
in the United Kingdom to be registered as a foreign practitioner in the Register of Veterinary Surgeons and to become to all intents a nember of the said Royal College.

(3.) For the purpose of this section a veterinary diploma is any diplonia, licence, certificate, or other document granted by any university, college, corporation, or other body in respect of veterinary surgery, and includes a licence or authority to a person to practise veterinary surgery granted by any department of or persons acting under the authority of the government of the country or place within or without Her Majesty's Dominions wherein the licence or authority is granted ; and a British Possession is any part of Her Majesty's Dominions out of the United Kingdon ; and a recognised veterinary diplona is a veterinary diploma recognised for the time being by the Council of the said Royal College as furnishing a sufficient guaranty of the possession of the requisite knowledge and skill for the efficient practice of veterinary surgery, and as entitling the holder thereof to practise veterinary surgery in the British Possession or foreign country wherein the diploma was granted.

(4.) If a person is refused registration as a colonial practitioner, or as a foreign practitioner, the Council of the said Royal College shall, if required by that person, state in writing the reason for that refusal, and if that reason be that the reterinary diploma held or obtained by hin is not a recognised veterinary diploma, that person may appeal to the Privy Council, and the Privy Council, after communication with the Council of the said Royal College and the appellant, may either dismiss the appeal or order that Council to recognise that veterinary diploma.

I4. The said charters of the Royal College of Veterinary Surgeons are hereby confirmed, and are ceclared to be and shall be in full force and virtue, except as far as the same are by this Act altered, or as the same are inconsistent with this Act, but not so as to prevent the making of any amendment thereof or addition thereto by any supplemental Royal Charter not being inconsistent with this Act.

I5.-(I.) Where at the passing of this Act any person practises and has continuously for not less than five years next before the passing of this Act practised veterinary surgery in the United Kingdom, but is not on the Register of Veterinary Surgeons, he shall be entitled, subject to the provisions of this Act, to be placed on a separate register under the heading of 'Existing Practitioners,' without examination, on such terms as to payment of fees, and as to other matters, as the Council of the said Royal College, with the approval of the Privy Council, direct.

(2.) On any person applying for registration under this section within one year after the passing of this Act, and thereupon, or within a reasonable time thereafter, producing to the Council of the said Royal College evidence of his title to registration by statutory declarations of himself and of other 
persons able to testify on his behalf, or such other eridence as that Council reasonably require, he shall be registered under direction of that Council accordingly.

(3.) If a person is refused registration under this section, the Council of the said Royal College shall, if required by him, state in writing the reason for that refusal, and he may appeal to the Privy Council; and the Privy Council, after communication with the Council of the said Royal College and the appellant, may either dismiss the appeal or order that Council to register the appellant under this section.

(4.) No person registered under this section shall be deemed to be a member of the said Royal College within the said Charters or this Act.

16. If after the passing of this Act any person not being a fellow or a member of the Royal College of Veterinary Surgeons takes or uses any name, title, addition, or description, by means of initials or letters placed after his name, or otherwise, stating or implying that he is a fellow or a member of the Royal College of Veterinary Surgeons, he shall be liable to a fine not exceeding twenty pounds.

I7.-(I.) If after the thirty-first day of December one thousand eight hundred and eighty-three any person, other than a person who for the time being is on the Register of Veterinary Surgeons, or who at the time of the passing of this Act held the veterinary certificate of the Highland and Agricultural Eociety of Scotland, takes or uses the title of veterinary surgeon, or veterinary practitioner, or any nane, title, addition, or description stating that he is a veterinary surgeon or a practitioner of veterinary surgery or of any branch thereof, or is specially qualified to practise the same, he shall be liable to a fine not exceeding twenty pounds.

(2.) From and after the same day a person other than as in this section mentioned shall not be entitled to recover in any court any fee or charge for performing any vetcrinary operation, or for giving any veterinary attendance or advice, or for acting in any manner as a veterinary surgeon or veterinary practitioner, or for practising in any case vetcrinary surgery, or any branch thereof.

18.-(1.) All powers vested in the Privy Council by this Act may be exercised by an Order of Council made by two or more of the Lords and others of Her Majesty's Most Honourable ''rivy Council.

(2.) An order made by the Privy Council under this Act may be made conditionally or unconditionally, and may contain such terms and directions as to the P'rivy Council secm just.

(3.) The Council of the said Royal College shall forthwith olscy any Oriler of the l'rivy Council under this Act, and observe and fulfil all conditions, terms, and directions therein containcel. 
I9. Fines and imprisonment under this Act may be recovered and imposed summarily, that is to say-

in England in manner provided by the Summary Jurisdiction Act 1848 and the Summary Jurisdiction Act 1879 and any Act amending either of those Acts ;

in Scotland before the sheriff or sheriff-substitute or two justices in manner provided by the Summary Procedure Act I864 and any Act amending the same;

in Ireland within the police district of Dublin metropolis in manner directed by the Acts regulating the powers and duties of justices of the peace for such district or of the police of such district, and elsewhere in Ireland before two or more justices of the peace in manner directed by the Petty Sessions (Ireland) Act 1851 and any Act amending the same.

A prosecution under this Act may be instituted by the Council of the Royal College of Veterinary Surgeons, but shall not be instituted by a private person without the written consent of the said Council.

20. Nothing in this Act shall affect the charter and supplemental charters granted by Her Majesty to the Royal Veterinary College in the years $\mathbf{I} 875$ and $\mathbf{I} \delta 77$ respectively, or any of the property, rights, powers, and privileges of that College thereunder.

\section{THE TITLE 'VETERINARY CHEMIST'}

I. February I 893 the Royal College of Veterinary Surgeons prosecuted Mr. Richard Henry Grover, pharmaceutical chemist, of Blandford, for an alleged infringement of the Veterinary Surgeons Act, sect. 17, by lraving published a book or pamphlet recommending certain reterinary medicines on which he has described himself as a 'pharmaceutical and veterinary chemist.' Counsel for the prosecution stated to the magistrates that the action had been brought "to have once for all the important question decided.' The College relied principally on a judgment given in a previous case (R.C.Y.S. $\because$. Robinson) by Justices Hawkins and Wills, who had held that in using the description 'veterinary forge' the defendant, who was a person not qualified under the Veterinary Surgeons Act, had professed to have some special skill in veterinary surgery, and they held, therefore, that he had infringerl the Act. The Blandford magistrates considered that the case was doubtful, and they dismissed the summons, but stated a case. The proprietors of "The Chemist and 
Druggist' had organised a subscription among chemists and druggists interested in the question, and the appeal came before a divisional Queen's Bench Court, and was heard by Justices Day and Lawrence on May $3 \mathrm{I}$, I $\$ 93$. A full report of the proceedings before the magistrates appears in 'The Chemist and Druggist,' February II, IS93, and the arguments heard by the Iligh Court and the judgment there delivered are reported in 'The Chemist and Druggist,' June 3, I893.

After hearing Mr. Poland for the College, the judges, without calling upon Mr. Bray, who appeared for the chemist, dismissed the appeal. Mr. Justice Day said: 'As far as I can see, it is competent for any person, whether he is a pharmaceutical chemist or no chemist at all, to publish his riews about animal diseases, and to nake suggestions as to what he thinks the best mode of treating them. I can see no possible objection to anything of the sort. ... I am not at all satisfied that because a man happens to be a chemist he is debarred from publishing a book of the kind. . . . Then it is said Mr. Grover may not do what he has done because he describes himself as a veterinary chemist. From the term "veterinary chemist," I should think a man was meant who laid himself out for the preparation of medicines used for horses, cows, \&.c. I am not aware that that is a part of veterinary surgery, or that that is practising as a veterinary surgeon. It is quite idle to present any argument of the kind. This man is, in my judgment, entitled to call himself a vetcrinary chemist in that sense, and he is also entitled to publish this book. With reference to the case that has been cited about the shoeingsmith, the only observation I shall make on the subject is that it is a different case.'

Mr. Justice Lawrence concurred, and the appeal was dismissed.

\section{TIIE CONTAGIOUS DISEASES (A.MIMALS) ACTS}

TuF Statutes and Orders of Council relating to the contagious discases of animals are very voluminous, and can only be hriefly alluded to herc. It may be mentioned that the Acts and Orclers, with a very complete alphaletical exposition of the duties arising under them, are published ofticially in a thick volume under the title of "I Iandlook for England, Wales, and scotland of the Laws and legulations relating to Contagious and Infectious Discases among Animals.' This work is published ly the fovernment printers at $2 s$, and may be obtained through any bookse'ler. 
The Acts apply to the whole of the United Kingdom.

The Acts and Orders formerly administered by the Agricultural Department of the Privy Council of Great Britain are now uncler the charge of the Board of Agriculture (created in ISS9 by the Foard of Agriculture Act, 52 \& 53 Vict. c. 30 ).

The Statutes comprise the Contagious Diseases (Animals) Acts of $S_{7} S$, ISS 4 , and ISS6; the Contagious Diseases (Animals) Act, Transíer of Parts of Districts Act, IS\&4; and the Pleuro-pneumonia Act of I 890 . Powers are given in the Acts to the Privy Council (now to the Board of $\Lambda$ griculture) to make and revoke orders in reference to other diseases than those mentioned in the Statutes themselves, and in reference to the importation of animals from foreign countries and the removal of animals in and from special districts. The diseases which are now legislated for under the Acts and Orders are the following :

Cattle Plague, also known as rinderpest.

Pleuro-pneumonia - that is to say, contagious pleuro-pneumonia of cattle.

Foot and Mouth Disease.

Sheep Pox.

Sheep Scab.

Glanders.

Farcy.

Swine Fever - that is to say, the disease known as typhoid fever of swine, soldier, purples, red disease, hog cholera, or swine plague.

Anthrax - that is to say, the disease known as anthrax, splenic fever, or splenic apoplexy of animals (i.e. cattle, sheep, and goats, and all other ruminating animals, and swine).

Rabies.

Sarcoptic mange of horses, asses, and mules. [This applies only to the Shetland Isles.]

The Acts are administered in Ireland by the Lord-Lieutenant and Privy Council of Ireland.

Local authorities are required to appoint inspectors under the Acts, and the Board of Agriculture also provides its own inspectors. Owners or persons in charge of animals affected with any of the diseases named above are required to give notice to a constable, who informs the inspector. The latter gives notice to the Board of Agriculture, and also serves certain notices on the owners or occupiers of the place where the disease occurs, and for a mile around. The Board of Agriculture can accept the notice from the local inspector, or can satisfy itself further, and then can make orders for slaughter and for compensation within certain limits; for prohibiting and regulating the movements of animals in the district ; for disinfection, dic. The owner of animals not only must give notice when he knows them to be affected with any of the diseases named above, but 
may give notice when he only suspects the existence of such a disease; and it then becomes the duty of the inspector to ascertain for himself what steps are necessary. The regulations enforced vary in different diseases, but generally it may be said that treatment is forbidden and slaughter is insisted upon in all cases. The regulations as to importation and transfer of animals are very voluminous, but need not here concern us.

\section{SALE OF HORSES}

THE law affecting the sale of horses (so far as it differs from the law concerning the sale of goods generally) has been digested as under by Judge Chalmers from the statutes, 'Act against the Buying of Stolen Horses' (2 \& 3 Phil. \& Mar. c. 7), and 'Act to avoid Horse-stealing' (3I Eliz. c. 12).

1. The sale of any horse, whether in market overt or otherwise, shall be roid as against the true owner thereof, unless such sale be made in accordance with the following rules.

2. When a stolen horse has been sold in market orert, and in accordance with these rules, the true owner may recover the same, if he nake claim thereto within six months of the theft, on tendering to any person who may have bought it in good faith, the price which he gave for the same.

3. The market authority in every horse fair or market shall cause a special open place to be marked out for the sale of horses.

4. There shall be a toll-keeper appointed for such place, who shall take tolls and kcep the place from ten before noon until sunset of each market day, and no tolls shall be taken except between the aforesaid hours.

5. No horse shall be sold or otherwise transferred, unless it has been exposed in the place of sale for one hour at least during the hours aforesaid,

6. When the toll is taken the partics to the sale or transfer shall be present before the toll-keeper, and the toll-keeper shall enter in a book, to be kept for that purpose, the names, surnames, and address of the parties, together with the colour and one special mark at least of the horse so sold or transferred.

7. The toll-keeper shall not enter the sale or transfer in his book unless he will take upon hinself perfect knowledge of the name, surname, and address of the person selling or transferring the horse, or unless the person so selling or transferring the horse is vouched for by a sufficient and credible jersnn known to the tull-kecper, who is personally acquainted with him, and knows his name, surname, description, and address. In the latter 
case the toll-keeper shall enter in his book the name, address, and description of the seller or transferrer, and of the person who vouches for him, and also the price, if any, given for the horse.

S. A note of the entry in the toll-keeper's book shall be given to the buyer, who shall pay the sum of twopence therefor.

9. Not later than the day after the conclusion of the fair or market the toll-keeper shall cleliver his book to the market authority, who shall cause a note to be made of the true number of all horses sold at the said fair or inarket.

I0. In these rilles the term 'horse' includes mare, gelding, colt, and filly; and 'toll-keeper' includes deputy toll-keeper, or book-keeper, when by usage no toll is taken.

II. When according to the usage of the market no toll is taken, the book-keeper shall be entitled to one penny for each sale or transfer entered in his book.

\section{THE VETERINARY CURRICULUM}

ALL persons who practise as 'veterinary surgeons' must be registered as such by the Royal College of Veterinary Surgeons; and the only course open for persons entering the profession now is to study at one of the veterinary colleges and pass the examinations of the Royal College, which is not itself an educating body.

A student, before presenting himself for the first professional examination, is required to pass the preliminary examination of the General Medical Council, or one recognised ly that body, and this should be passed before he begins his professional studies.

The shortest period of study necessary to obtain the diploma is four sessional years, which implies that a student must attend the classes, \&.c., of one or more of the recognised colleges during a session of not less than thirty weeks in each of the four years.

There are four professional examinations, one at the end of each sessional year, conducted by the Examining Board of the Royal College of Veterinary Surgeons. Examinations are held three times during the year, one in March, April, or May, one in December or January, and a third in July, provided that not less than eighteen candidates in any one class are presented for examination.

The fee for each professional examination is $5 l$, and for rc-cxamination after rejection $3 \% 35$.

In addition, on passing the final examination a registration fee of 1 . is demanded. 
The subjects of the professional examinations, which are both suritien and oral, are as follows :-

First.-Examination A.

I. Anatomy of domesticated animals (Bones, Ligaments, Joints).

2. Chenistry and Elementary Physics.

3. Biology (Elementary Zoology and Botany).

Second.-Examination B.

I. Anatomy of domesticated animals.

2. Histology and Physiology.

3. (Oral only.) Stable management and manipulation of domesticated animals; principles of shoeing.

Third.-Examination C.

I. Morbid Anatomy, Pathology, and Bacteriology.

2. Materia Medica, Pharmacy, Therapeutics, and Toxicology.

3. Veterinary Hygiene and Dietetics.

Fourth. - Examination D.

I. Yrinciples and Practice of Veterinary Medicine and Surgery ; Clinical Medicine, Surgery, and Obstetrics (horse).

2. Principles and Practice of Veterinary Medicine and Surgery ; Clinical Medicine, Surgery, and Obstetrics (other domesticated animals); Meat Inspection.

All letters in regard to the examinations should be addressed to the Secretary, R.C.V.S., ro, Red Lion Square, London, IV.C.

\section{METHYLATED VETERINARY PREPARATIONS}

THERE is an impression which is largely prevalent that methylated spirit may legally be used in the preparation of veterinary medicines. This is not the case, and indeed a good many fines have been imposed on chemists who have had some methylated veterinary preparation in stock. In Alpe's ' Iandy IBouk of Medicine Stamp Duty,' the following useful advice on this subject is given.

The Commissioners of Inland Revenue, by a circular to chemists and druggists, in $189 \mathbf{1}$, intimated to the trade that methylated spirit might be used in the preparation of the following articles:- Iydrate of chloral, soil] liniment, compound camphor liniment, aconite liniment, belladonna liniment.

The Commissioners also allow methylated spirit to be used in the manufacture of horse and cattle medicines so compounded as not to he "afalile of leeing used internally as medicines hy human beings. In each case a special application must he made, and the formula of composition stited, and methylated spirit may not le used in the manufacture of such 
preparations until the special permission of the Commissioners has been obtained, and such security given (usually by bond) as they may require for the proper use of the methylated spirit. Such preparations as mustard liniment, iodine liniment, tincture of arnica, tincture of benzoin, \&c., which contain drugs commonly prescribed for internal use by human beings, are not allowerl to be prepared with methylated spirit ; and cattle medicines, if reasonalsly capable of being used for human beings, fall under the same category. l'ermission would be denied with regard to tincture of aloes or of camphor, but it would probably be granted in the case of blisters, Leeming's Essence, strong opium dranghts, corrosive sublimate applications, \&.c. Formulas of preparations which would be allowed are quoted in the book named.

\section{ADIIIVISTRATION OF POISONOUS DRUGS TO HORSES ACT OF IS76}

THIS Act renders any person liable, on conviction, to a penalty of $5^{\prime}$., or one month's imprisonment, for administering or causing to be administere 1 to or taken by any horse, cattle, or domestic animal any poiscnou; or injurious drug or su'stance. Applies to England and Wales only. 


\section{N D E X}

ABS

A BSCEsses (see liounds, \&.c.) Alteratives, balls and powders, 23

Anxsthetics, how to give, I I

Arsenic for horses, 29; for dogs, 29

BleEnixg, method and effect of, 8

Blisters, 37, 30S, 309

Iran mash, 309

Broken knees (see IIorse)

CAI.F meal, 3 io

Calves, corclial, 40

- diarrhœa, 159, 3 I 4

- hoose or husk, IS4, 3 IS

- lice, I9I

- meal or milk substitute, 310

- scours or skit, 40

- thrush, igs

Canadian oil, 310

Canaries, asthma cure, $30 \mathrm{~S}$

- bright plumage, jor

Castration oil, 310

('ataract, 264

rats, to kill, 13

Cattle, alortion, $3-6$

- alscesies (see Wounds, S.c.)

- alministration of medicine, sc., 3, 157

- liarh legg. 5j, $30 S$

- Llecrling, 8
C.AT

Cattle, brain, inflammation, 194

- bronchitis, IS2

- bull-burnt, ISI, 309

- calving drench, 310

- cancer ointment, 310

- carbuncle powder, 310

- chloroform administration, I I

- cleansing, $3 \mathrm{~S}, 177$

- colchicum poisoning, $3 \mathrm{I} 2$

- cold, I94

- constipation, 154

- cordial drench, 40

-. cough, 195

- counter-irritant, $3 \mathbf{I} 3$

- cow-pox, I67

— cuc? balls, 313

- diarrhoea, 40, 159

- draughts, use and preparation of, 3

- drenches, $3^{S}, 40$

- drying off, I $S_{2}$

- epizootic aphthr, IS5

- cye discases, 259

- fardel-bound, 154

- fellow-chine, 316

- fever, (milk) I69, (jarturi. cnt) 174

- foud, 310

- foot and mouth disense, IS5

- fouls, lotion for, 320

- garget, 179 ; oils, 317 ; ointment, 317

- gripe drencli, 3 rS

- hroose (sec Calves) 
CAT

Cattie, hoven or tympanites, I62, 3 IS

- intestinal tubercle, 197

- jaundice, I6I

— joint ill, $1 S_{3}$

- kidneys, inflammation, 194

- lice, 52, I9I

- lumbago, 316

- mammitis, I79

- milk, (blue) 197, (bloody) 197

- - fever, I69; powder, 320

- - suppression of, 167,195

- murrain, I $\$_{4}$

- parturient fever, 174

- piles, 315

- placenta, retention of, 177

- pleuro-pneumonia, ISS

- pulse, 6

- purgatives, 321

- red-water, I50

- rheumatism, I $8_{3}$

- ringworm, 53, 193

- scours or scouring (see Calves)

- spice, 310

- stoppage, 154

- tapeworm, I98

- temperature, 5

- thrush (see Calves)

- tonics, 26, 39

- tuberculosis, 195

- tympanites or hoven, 162

- udder, inflammation of, 179

- ulcers (see Wounds, \&c.)

— warbles, 55, 323

- warts, 32 I

- wounds, oil for, 37

- yellows, I6I

Charges, $3 S$

Cheshire red bottle, 3II

Chlorodyne (veterinary), 3 I I

Chloroform, adninistration of, I0

Cleansing drinks, 179

Colchicum poisoning, 312

Colic (see Gripe)

Condition powders, 22

Contagious Diseases (Animals) Acts, 332
I)OG

Cough balls, powders, S.c. (sce animal)

Counter-irritant, 3 I 3

DEMEDOX folliculorum, 50

Devonshire oils, 313

Diapente, 3 I 4

Diuretics, 24

Dogs, abscesses and ulcers (sce Wounds, \&.c.)

- administration of medicine, ¿c., 4, $22 \mathrm{~S}$

- alterative, $4 \mathrm{I}$

- astringent, 42

- bleeding, ro

- canker, $25 \mathrm{I}$

- cataract (see Eye)

- chloroform, administration of, II

- chorea, 235

- constipation, 242

- cough, 42, 313

- deafness, 253

- dentistry, 256

- distemper, 4I, 229, 3 I 4

- eclampsia, 242

- eczema, 239

- eye diseases, 260

- fits, 250

- gastritis, 246

- holding, 227

-- hot feet, 254

- influenza, 247

- jaundice, 244

- killing, ${ }_{3} 3$

- lungs, inflammation, 254

- mange, 237

- pills, alterative, 4I ; astringent, 42; cough, 42; purgative, 42 ; tonic, 42 ; worm, 42

- pnetrmonia, 254

- rabies, 254

- rheumatism, $2+5$

- sickness, 226

- tears, overflow (see E.ye)

- teeth extraction, 256

- temperature, 5,227

- thermometer, use of, 227 
DOG

Dogs, tonic, 42

- wash for, 3 I 4

- worms, 248

- wounds and sores (see Wounds, \&c.)

Dose table, 302

Drenching, 157

Driffield oils, 315

Ellimas's horse-balls, is

Eye diseases, 259

- cataract, 264

- eyeball, dislocation, 265

- eyelid, torn, 262

- grape or staphyloma, 263

- humours of, diseases, 264

- inflammation, 259

- tears, overflow of, 265

- water for horses, 316

FELLOW-CHINE, 316

Fleming's tinct. of aconite, 316

Fly and maggot oils, 316

- powder, 317

Foot and Mouth disease (sce Cattle)

Foot-rot applications, 317

Formula, miscellaneous, 308

Fowls (see Poultry)

(iAI)FLY, $\mathrm{II}_{3}$

Gapes, 300, 317

Guese, 297

Gripe merlicines, $33,163,31 \mathrm{~S}$ (sce under various animals)

IIEALING Jution, 3is

Hleaves remedy, 3 is

lloof ointments, 43

Horse, aluscesses and ulcers (see Wounds)

- acne, 109

- arlministration of meslicine, 1

- age and the teeth, 147

- alteratives, 23
HOR

Horse, appetising powder for, 308

— balls, 2, 31S ; (condition) 19, 321

- bladder, inflammation of, Ic6

- bleeding, $\mathrm{S}$

- blisters, 37, 30\$, 300

- bng-spavin (see Spavin)

- bots, II 3

- bran mash, 309

- broken knees, 36, 315, 320

- broken wind, 9 I

- bronchitis, $s_{3}, 309$

- canker, 135

- capped hock, 143

- catarrh, 77

- charges, $3 S$

- chloroform, administration, I I

- cold, 77

- colic, 59, 65, 312

- condition powders, 22, 312

- condition, show, 20

- constipation, 69

- cordial balls, 25

- corna, 126

- cough, 79, (balls) 31, 313 , (electuary') 33, (draughts) 313 , (powders) 3I 3 , (tonic) 26, (treatment for), $3 \mathrm{I}$

- cracked heels, 43,125

- curb, 136, 314

- cystitis, 106

-. dentition, 147

- diabetes insipidus, is

-- diapente, 314

- cliarrhea, 40, 70

- draughts, use of, I

- cczema, 107

- emetic, non-use of, 2

- enteritis, 76

- cye discase (see Eyc)

- eje water, 316

- false quarter, 133

- Tarcy, 115

- fever, 97, (balls) 26, 316, (draught) 316 (sec Laminitis and Mud-f(ver)

- fistula, 277

- flies on, 46 
IIOR

Horse founder, 142

- galls, saddle and harness, 36 , $27 \mathrm{~S}$

gastritis, 76

- glanders, II 4

- grease, ointment for, $3^{17}$

- gripes, 33

- harness galls, $3^{6}$

- heels, stopping for, 324

- - cracked, 43, I25

- hoof, ointment, 43

- impaction of the bowels, 70

— influenza, S9, 319

- jaundice, 74

- knees, 36, 315, 320

- lameness in, I I9; liniments, 319

- laminitis, 142

- legs, swollen, 3 I

- liniments, 35

- liver, inflammation of, 72

- lungs, congestion (see l'neumonia)

- mallenders, 107

- mange, $4 \mathrm{~S}$

- megrims, 104

- mud-fever. 99

- navicular disease, 135

- nettle rash, I Io

- physic mass, preparation, ¿.c., I6, 324

-- pink eye, 89, 319

- pityriasis, 108

- pleurisy, 86

- pneumonia, 84-95

- poisons, administration, 337

- poll-evil, 277

- polyuria, I 18

- prurigo or pruritus, Io8

- purgative powders, 32 I

- quittor, 274

- rheumatism, I4I

- ring-bones, I2S

- ringworm, 53

- roaring and whistling, 93

- saddle galls, $36,27 \mathrm{~S}$

— sale of, 334

- sallenders, 107

— sandcracks, 134
MAL

IIorse, scedy-toe, 135

- shivering, 104

- show condition, 20

- side bones, I 3 I

— sitfasts, 279

- sore shins, 143

- - shoulders, 2 So

- - throat, $\mathrm{IO}^{3}$

- spavin, 132

- splints, 143

- sprains and bruises, 35, I 37

- staggers, 105

- stomatitis, 75

- strangles, IOI

- surfeit, I IO

- tail, itchy, 109

- teeth, Sic., 147

- temperature, 5

- thoropin, 136

- throat liniment, 322

- thrush, I 38

- tice or entice, 3 IS

- tonics, 26, 315

- tread or over-reach, 133

- ulcers, 272

- warbles, 55, 323

- warts, 44

- water farcy, i i 5

- whistling, 93

- white-bottle, 35

— white oils, 35

- wind-galls, I40

— worms, 52, II 1, 323

- wounds, 37

Huish's milk-syphon, I79

LAmbing (see Sheep)

Leeming's Essence, 319

Leg, black, 53

Legs, swollen, 3 I

Lice (see Cattle, Sheep, S.c.)

- oils, 52

Liniment, white stimulating, 322

Lotion, sedative, 54

MagGot oils, 316

Mallein, I I

Malt mash, 320 
MA.S

Mange, 48, 320

Mashes, bran, 309 ; malt, 320

Methylated preparations, 336

Milk substitute (calves), 3 10

- syphon, 179

Mithridate, 320

Mud-fever (see under animal)

Newmarket Physic Paste, 324

OAtaneal gruel, 320

Oil, Canadian, 3 Io

- castration, 3 IO

Oils, Devonshire, 314

- Driffield, 315

- fly and maggot, 316

- white, 35, 317

Ointment, blistering, $30 \mathrm{~S}$

- for grease in horses, 320

- green, 317

- horses' knees, 320

- for sores, chafes, 320

- for warts, 32 I

Oxen (see Cattle)

PIIEASANTs, cold and roup, 300

- gapes, 300, 3 I 7

- scrofulous cliseases, 301

Pigeons, canker, $29 \mathrm{~S}$

- diarrhœa, $29 S$

- nloulting, $29 \mathrm{~S}$

- parasites, kc., 298

- pouters, $29 \mathrm{~S}$

- roup, $29 S$

- scouring, $29 \mathrm{~S}$

- scrofula, 298

- sore cyes, 298

- spice balls for, 300

- swelling of the crop, 297

- tonic pills for, 322

- vertigo, 298

- wasting, $29 \mathrm{~S}$

I'igs, al)scesses (see Wounds, S.c.)

- administration of medicine, S.c., 3

- anthrax, 222

- blaine or stye, 224

- bleeding, 10
POU

Pigs, bruises, sores, 224

- catarrh, nasal, 224

- cod-liver oil for fattening, 225

- colic, 222

- cough, 220

- cramp, 322

- diarrhœa, 220, 322

- evil, 217

- eye diseases, 222

- fattening, 225

- fever, parturient, 216 , (swine) 216

- gripes or colic, 222

- joint-ill, 217

- loss of tail, 220

- lung, inflammation, 220

- maggots in the ear, 222

- nasal catarrh, 224

- parturient fever, 216

- powders, 32 I

- protruded rectum, 2 I 7

- quinsy or strangles, 222

- rheunatism, 2 I 7

- scours, 220

- sniffles or snuffles, 224

- sprains, sores, and bruises, 224

- strangles or quinsy, 222

- stye or blaine, 224

- swine fever, 216

- tail, loss of, 220

- Hlcers (see Wounds, \&c.)

- worms, 224

- wounds, s.c. (see Wounds)

Pills (see various animals)

l'osological table, $3 \mathrm{O} 2$

Poultry (see names of birds)

- abortion, 286

- apoplexy, $2 S$

- baldness and whitecomb, $2 S_{7}$

- black rot, $2 S_{7}$

- bronchitis, $2 \$ 7$

- bumble foot, $2 S_{7}$

- canker, $2 S S$

- catarrli, $2 S S$

- chulcra, $2 \mathrm{SS}$

- crainp, $2 S_{9}$

- frop-bound, $2 \$ 9$

- crop soft or swelled, $2 \$ 9$ 
POU

Ponltry, croup, $2 S 9$

— debility, 290

— diarrhœa, 290

- Douglass mixture, 290

- dysentery, 290

- egg-bound, 290

- elephantiasis, 290

- feather eating, 29I

- gapes, 29I

- giddiness, 292

- indigestion, 292

- leg weakness, 292

— lice, 296, 297

- liver disease, 292

- loss of feathers, 292

- moulting, mixture for, 290

- paralysis, 293

- parasites, 293

— pip, 293, 296, 297

- rheunatism in, 293

- roup, 293, 322

- soft eggs, 294

- thrush, 294

— tuberculous diseases, 295

- vertigo, 295

- worms, 296

I'ulse, how to take, varying conditions, \&.c., 6

QCITTOR, 2\%4

Restitutiox fluid, 319

Ringworm ointment, 54

Rowels, 7

SEToxs and rowels, use of, 7

Sheep, abscesses and ulcers (see Wounds, \&ic.)

- administration of medicine, 3

- anæmia, 199

- anthrax, 200

- antiseptic lotion, 315

- bleeding, I I

- blood diseases, 199

- bots, 200

- consumption, 212
VET

Sheep, cough, 2 I 2, 322

- diarrhoca, 213

— dips, formulie, \&.c., 56, 206

- fever, 322

- fukes, 2 Io

- fly, 20S, 316

- foot-rot, 48, 2 I 4

- garget, 2 I 2

— gid, 2 I 3

- giddiness, 2 I 2

-- grub in the head, 200

- hoven, $2 \mathbf{I}_{3}$

- husk, $20 \mathrm{I}$

- lambing, 202, (drench) jI9, (oils), 3 I9

- lice, 204

-- parasites, 204, 208

- poke or chocker, 199

- pox, 2 I I

- red water, 213

- ringworm, 53

- rot, flukes, I\$9

- scab, 203

- soothing mixture, 3 I 5

- stimulating mixture, 315

- sturdy, 213

- thrush, 202

- ticks, 204

- turnsick, 2 I $_{3}$

— udder (swollen), 2 I 2

- worm oil, 323

- wounds (see Wounds)

Sprains and bruises, 35, I 37

Spring medicines, 5 I

TEMIERATURE, how to take, range of, \&c., 5

Tinct. opii aquosa, 322

Tuberculosis, I95, 2 I 2

Turkeys, 295

Tuson's horse-balls, formula, I 7

ULCERS, 272

Veterisary Chemist, title, $33 \mathrm{I}$

- curriculum, 335 
Y'ET

Veterinary Surgeons Act (44 \& 45 Vict. c. 62$), 325$

WARBLes, 55, 323

Warts, ointment for, 32 I

- treatment of, 45

White flaw, 53

Worm balls, \&c., 52, I I I, 323

Wounds, sores, \&c., 267

- abscesses, 27 I

- balsam for, 324

- collar galls, 278

- dusting powder, 324

- fistula, 277

- fractures, 28

\section{WOU}

Wounds, galls, sadule, and harness, 36,278

- grease in horses, 282

- incised wounds, 268

- lacerations, 269

- open joints, 28I

- poll-evil, 277

- quittor, 274

- sinus ulcers, or fistulx, 274

- sitfasts, 279

- sore shoulders, 280

- stone, 324

— ulcers, 272

- woundstone for, 324 

University of British Columbia Library Sools DATE DUE UEC 1 E TS:
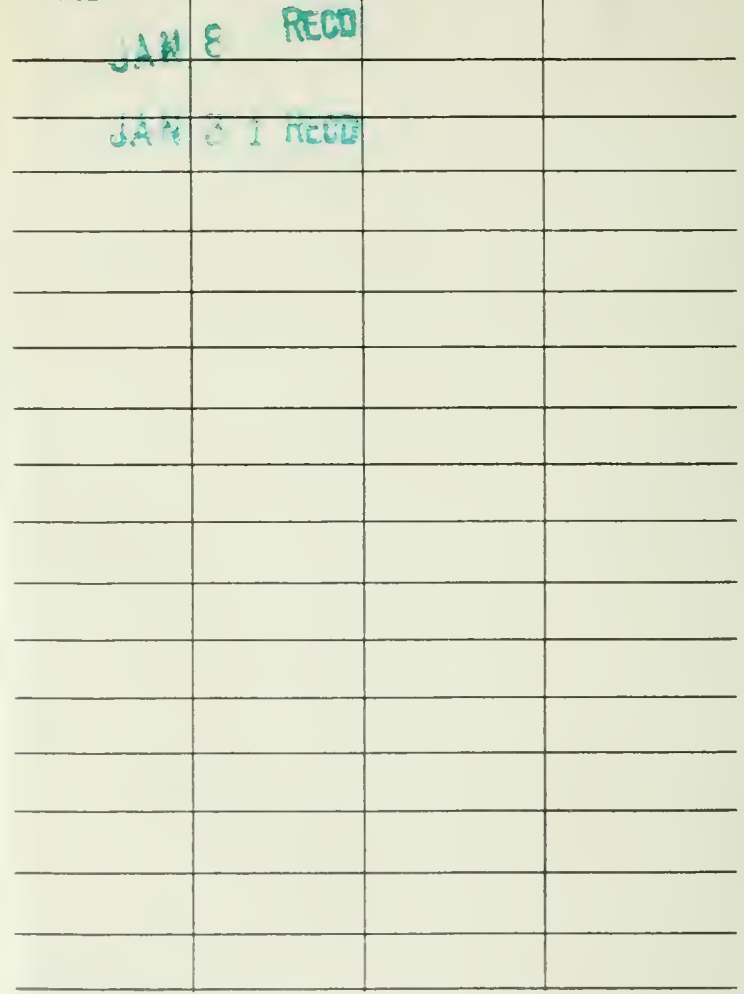

FORM No. 310 


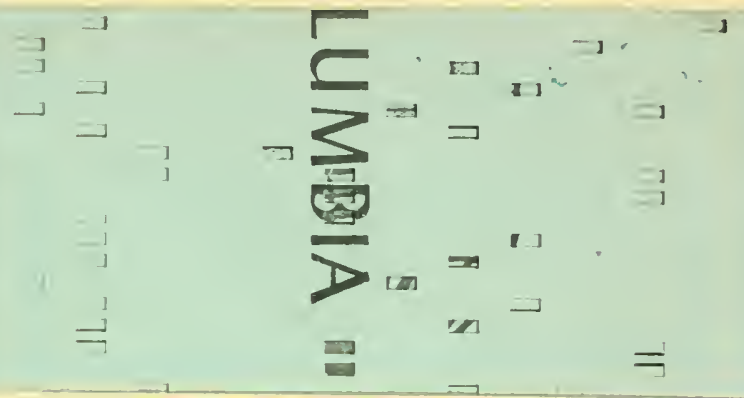




$$
\text { wher wat }
$$<smiles>C#CC1=CC=CC(C)C1C#C</smiles>

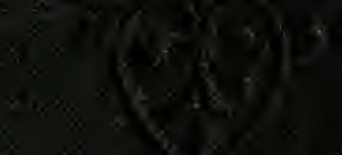

8 